\title{
Juvenile Rheumatoid Arthritis
}

\section{Diagnostic Criteria}

Juvenile rheumatoid arthritis (JRA) (JA) has been the diagnostic label applied to any child whose arthritis is of unknown origin, begins under age 16, and persists for a minimum of 6 weeks. ${ }^{1,2}$ Arthritis is defined as swelling or both pain and limitation of motion in at least one joint. "Unknown origin" means that the physician has considered and excluded all other diseases, both common and unusual, that may cause or be associated with arthritis (Table 3.1). These "classification" criteria, adopted by a panel of experts (the JRA Criteria Subcommittee of the American Rheumatism Association-ARA, now the American College of Rheumatology-ACR), were extraordinarily successful in helping physicians distinguish arthritic disorders from other disorders in childhood. After 5 years, only $3 \%$ of children diagnosed as JRA by these criteria turned out to have some other disease. Interest in childhood arthritis was stimulated by publication of these criteria, and they helped to bring about the end of an era in which children with arthritis were frequently misdiagnosed and subjected to long hospitalizations, unnecessary surgery, harmful immobilization, and inadequate or inappropriate drug therapy.

\section{Subtypes of JRA}

During the past decade, it has become apparent that these criteria actually identify a consortium of different disorders, a panache with different genetic susceptibility determinants, environmental offsets, pathology, prognoses, and clinical patterns (Table 3.2). ${ }^{2-10}$ As a result, these criteria failed to provide a strong relationship between diagnosis and prognosis in a given pa- 
Table 3.1. Clues to Some Unsual Causes of Arthritis in Children

\begin{tabular}{|c|c|c|}
\hline Clue & Diagnosis & Diagnostic Procedure \\
\hline $\begin{array}{l}\text { Strong family history of hip } \\
\text { replacements in young } \\
\text { individuals with degen- } \\
\text { erative hip disease }\end{array}$ & $\begin{array}{l}\text { Spondyloepiphyseal } \\
\text { dysplasia }\end{array}$ & Lateral spine $x$-ray \\
\hline $\begin{array}{l}\text { Pain in foot with atrophy of } \\
\text { extremity }\end{array}$ & Osteoid osteoma & Bone scan, CT Scan \\
\hline $\begin{array}{l}\text { Recurrent brief attacks of } \\
\text { severe monarticular } \\
\text { arthritis with slight } \\
\text { ecchymosis }\end{array}$ & Synovial hemangioma & $\begin{array}{l}\text { CT scan, Phlebolith on } \\
\text { x-ray (late) }\end{array}$ \\
\hline $\begin{array}{l}\text { Recurrent brief attacks with } \\
\text { rash overlying affected } \\
\text { joints }\end{array}$ & $\begin{array}{l}\text { Familial Mediterranean } \\
\text { fever }\end{array}$ & $\begin{array}{l}\text { Observation of rash } \\
\text { and attack }\end{array}$ \\
\hline $\begin{array}{l}\text { Punched-out lytic lesions in } \\
\text { metacarpals and multiple } \\
\text { bones }\end{array}$ & $\begin{array}{l}\text { Child abuse with trauma- } \\
\text { tic pancreatitis }\end{array}$ & $\begin{array}{l}\text { Serum amylase/lipase } \\
\text { Bone scan for hidden } \\
\text { lesions }\end{array}$ \\
\hline $\begin{array}{l}\text { Initial onset of hip pain } \\
\text { while doing a split }\end{array}$ & $\begin{array}{l}\text { Acute chondrolysis or } \\
\text { transient demineraliza- } \\
\text { tion of the hip }\end{array}$ & Repeat hip x-rays \\
\hline $\begin{array}{l}\text { Thick hyperostotic eryth- } \\
\text { ematous proximal } \\
\text { phalanges with periostitis } \\
\text { and bone chards }\end{array}$ & $\begin{array}{l}\text { Child abuse-beating } \\
\text { across fingers with a } \\
\text { ruler }\end{array}$ & X-ray \\
\hline $\begin{array}{l}\text { Exposure to plant thorns } \\
\text { shortly before the onset of } \\
\text { monarticular arthritis }\end{array}$ & Plant-thorn synovitis & Joint exploration \\
\hline $\begin{array}{l}\text { Bizarre (traumatic) lesions } \\
\text { of fingers and toes with } \\
\text { family history of "extra- } \\
\text { ordinary brave deeds" }\end{array}$ & $\begin{array}{l}\text { Congenital indifference to } \\
\text { pain }\end{array}$ & History \\
\hline $\begin{array}{l}\text { Low-grade pain in the up- } \\
\text { per tibia in young boys } \\
\text { with appearance of } \\
\text { periosteal new bone }\end{array}$ & Stress fracture & X-ray, bone scan \\
\hline \multicolumn{3}{|l|}{$\begin{array}{l}\text { Recurrent episodes of } \\
\text { arthritis }\end{array}$} \\
\hline $\begin{array}{l}\text { with chickenpoxlike } \\
\text { rash }\end{array}$ & $\begin{array}{l}\text { Mucha-Habermann } \\
\text { disease }\end{array}$ & Lesion biopsy \\
\hline $\begin{array}{l}\text { with painful plaque } \\
\text { rash and fever }\end{array}$ & Sweet's syndrome & Lesion biopsy \\
\hline
\end{tabular}


Table 3.1. Clues to Some Unsual Causes of Arthritis in Children* (Continued)

\begin{tabular}{|c|c|c|}
\hline Clue & Diagnosis & Diagnostic Procedure \\
\hline $\begin{array}{l}\text { Recurrent episodes of } \\
\text { arthritis: } \\
\text { with fever and ery- } \\
\text { sipeloid rash of lower } \\
\text { extremities }\end{array}$ & $\begin{array}{l}\text { Familial Mediterranean } \\
\text { fever }\end{array}$ & $\begin{array}{l}\text { History; trial of } \\
\text { colchicine }\end{array}$ \\
\hline $\begin{array}{l}\text { with erythema nodo- } \\
\text { sum or pyoderma }\end{array}$ & $\begin{array}{l}\text { Inflammatory bowel } \\
\text { disease }\end{array}$ & $\begin{array}{l}\text { GI radiographs, } \\
\text { colonoscopy }\end{array}$ \\
\hline $\begin{array}{l}\text { Pauciarticular arthritis with } \\
\text { history of unusual skin } \\
\text { lesion ("bug bite") the } \\
\text { month before }\end{array}$ & Lyme arthritis & $\begin{array}{l}\text { History } \\
\text { Serology; Western } \\
\quad \text { Blot }\end{array}$ \\
\hline $\begin{array}{l}\text { Arthritis with large post- } \\
\text { auricular lymph nodes }\end{array}$ & $2^{\circ}$ syphilis & $\begin{array}{l}\text { Serology; dark field of } \\
\text { mucous membrane } \\
\text { lesions }\end{array}$ \\
\hline $\begin{array}{l}\text { Arthritis with embolic skin } \\
\text { lesions and tenosynovitis }\end{array}$ & Gonorrhea & $\begin{array}{l}\text { Cervical/urethral/ } \\
\text { synovial fluid } \\
\text { cultures }\end{array}$ \\
\hline $\begin{array}{l}\text { Arthritis with intermittent } \\
\text { refusal to bear weight, } \\
\text { severe pain, anemia, } \\
\text { transient episodes of } \\
\text { severe bone pain }\end{array}$ & $\begin{array}{l}\text { Leukemia or other } \\
\text { malignancy }\end{array}$ & $\begin{array}{l}\text { Bone marrow, abdo- } \\
\text { minal ultrasound; } \\
\text { chest x-ray; MRI; } \\
\text { catecholamines }\end{array}$ \\
\hline $\begin{array}{l}\text { Arthritis that starts with } \\
\text { bent thumbs within a few } \\
\text { months of birth }\end{array}$ & $\begin{array}{l}\text { Familial hypertrophic } \\
\text { synovitis }\end{array}$ & $\begin{array}{l}\text { Family history, } \\
\text { biopsy }\end{array}$ \\
\hline $\begin{array}{l}\text { Arthritis with pyoderma } \\
\text { gangrenosum and no } \\
\text { systemic disease }\end{array}$ & $\begin{array}{l}\text { "Streaking leukocyte } \\
\text { factor" }\end{array}$ & $\begin{array}{l}\text { Serum enhancement of } \\
\text { WBC random } \\
\text { mobility }\end{array}$ \\
\hline $\begin{array}{l}\text { Arthritis with rash appear- } \\
\text { ing in newborn period }\end{array}$ & $\begin{array}{l}\text { Congenital arthropathy } \\
\text { with rash }\end{array}$ & $\begin{array}{l}\text { History; skin or liver } \\
\text { biopsy }\end{array}$ \\
\hline $\begin{array}{l}\text { Bizarre posturing of hand or } \\
\text { foot }\end{array}$ & $\begin{array}{l}\text { Reflex sympathetic } \\
\text { (neurovascular) } \\
\text { dystrophy }\end{array}$ & Physical examination \\
\hline $\begin{array}{l}\text { Monarticular arthritis with } \\
\text { night pain at rest, ex- } \\
\text { quisite response to aspirin }\end{array}$ & Osteoid osteoma & Bone scan; CT scan \\
\hline $\begin{array}{l}\text { Sudden episodes of flushing } \\
\text { and fever in an infant }\end{array}$ & Solitary mastocy toma & Physical examination \\
\hline
\end{tabular}

* See Chapter 2 for detailed discussion. 
Table 3.2. The Spectrum of Childhood Arthritis

\begin{tabular}{|c|c|c|c|c|}
\hline & \multicolumn{2}{|c|}{ Systemic } & \multicolumn{2}{|c|}{ Polyarticular } \\
\hline Type of onset & \multicolumn{2}{|c|}{$\begin{array}{l}\text { Spiking fever } \\
\text { Rheumatoid rash } \\
\text { Hepatosplenomegaly } \\
\text { Lymphadenopathy } \\
\text { Polyserositis } \\
\text { Myalgia, arthralgia } \\
\text { Leukocytosis, anemia }\end{array}$} & \multicolumn{2}{|c|}{ Symmetrical arthritis } \\
\hline $\begin{array}{l}\text { Pattern of joint } \\
\text { symptoms }\end{array}$ & $\begin{array}{l}\text { Same as } \\
\text { pauciarticular } \\
\text { JRA } \\
\text { (40\% of } \\
\text { systemic } \\
\text { onset } \\
\text { patients) }\end{array}$ & $\begin{array}{l}\text { Same as } \\
\text { polyarticular } \\
\text { JRA } \\
(60 \% \text { of } \\
\text { systemic } \\
\text { onset } \\
\text { patients })\end{array}$ & $\begin{array}{l}\text { Arthritis involvi } \\
\text { extremints (wrists, } \\
\text { shoulders, hip } \\
\text { feet, jaw, and } \\
\text { lumbodorsal s }\end{array}$ & $\begin{array}{l}\text { ing upper and lower } \\
\text { han small and large } \\
\text { s, knees, ankles, } \\
\text { neck); but not } \\
\text { pine }\end{array}$ \\
\hline Rheumatoid factor & Negative & Negative & Negative & Positive \\
\hline $\begin{array}{l}\text { Course of joint } \\
\text { disease }\end{array}$ & Remitting $-40 \%$ & $\begin{array}{l}\text { Remitting with } \\
\text { scarring- } \\
35 \% \\
\text { Severe, } \\
\text { unremitting } \\
\text { and } \\
\text { destructive- } \\
25 \%\end{array}$ & $\begin{array}{l}\text { Remitting } \\
\text { May "burn out" }\end{array}$ & $\begin{array}{r}\text { Persistent chronic } \\
\text { and destructive }\end{array}$ \\
\hline $\begin{array}{l}\text { Age at onset } \\
\text { (median) }\end{array}$ & 5 years & 5 years & 3 years & 12 years \\
\hline Sex ratio & $M=F$ & $M=F$ & $\mathrm{~F}>>\mathrm{M}$ & $\mathrm{F}>>>M$ \\
\hline $\begin{array}{l}\text { Antinuclear anti- } \\
\text { body }\end{array}$ & Absent & Absent & + in $25 \%$ & + in $75 \%$ \\
\hline HLA-associated & ? & ? & DPw3 & DR4 \\
\hline Uveitis & Rare & Rare & Rare & Absent \\
\hline Comments & $\begin{array}{l}\text { Systemic } \\
\text { manifestations u } \\
\text { ultimately remit } \\
\text { even if } \\
\text { arthritis continu }\end{array}$ & $\begin{array}{l}\text { sually } \\
\text { es }\end{array}$ & $\begin{array}{l}\text { Extra-articular } \\
\text { manifesta- } \\
\text { tions } \\
\text { generally } \\
\text { mild }\end{array}$ & $\begin{array}{l}\text { Childhood onset } \\
\text { of classical } \\
\text { adult RA } \\
\text { Subcutaneous } \\
\text { rheumatoid } \\
\text { nodules } \\
\text { common }\end{array}$ \\
\hline
\end{tabular}

(Modified with permission from Wedgwood and Schaller, Hosp Prac, June 1977.) 
Table 3.2. The Spectrum of Childhood Arthritis (Continued)

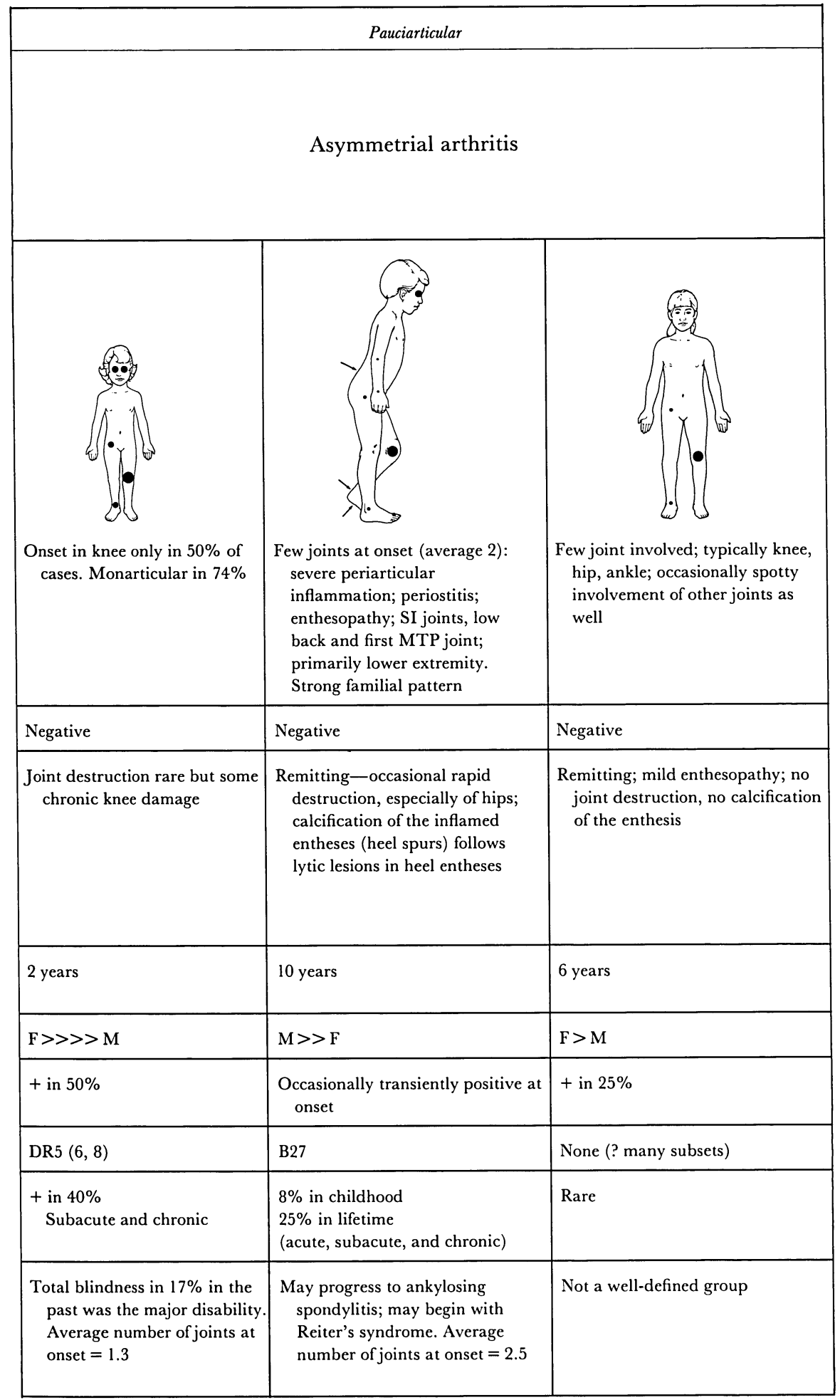


tient. It had been noted by Still in $1896^{11}$ and was reemphasized by Green in $1940^{12}$ and many subsequent authors ${ }^{2-10}$ that there were great differences between children who had spiking fever to $106^{\circ} \mathrm{F}$ daily, those children who had severe polyarticular disease but no systemic manifestations, and those whose arthritis was limited to one or a few joints. Accordingly, in 1977, the ARA-JRA diagnostic criteria were modified to divide patients with JRA into three onset subtypes: systemic (prolonged high fever), polyarticular (five or more affected joints; all cervical spine and all carpal or tarsal joints on one hand or foot count as only one each), and pauciarticular (four or fewer joints). ${ }^{13}$ By general agreement, the onset subtype is determined 6 months after the beginning of disease. Several studies have shown that $75-85 \%$ of children will either remit or remain in that subset 5 years later. Onset subtypes are convenient for study and for clinical trials, but they have less significance for the individual patient than the current pattern of disease and its course. ${ }^{14}$

It has also become apparent that all forms of "childhood" arthritis may begin at any age. ${ }^{15}$ The rarity of the rheumatoid-factor-positive ("seropositive") adult form of rheumatoid arthritis (RA) in childhood has merely served to cast the subsets of seronegative arthritis in sharper focus in the population of arthritic children. New understandings from the study of arthritis in childhood may be extended to seronegative arthritis in adults and vice versa. ${ }^{16}$ Subsets created by age, sex, or counting joints are only a temporary way of separating out various arthritic diseases. If diagnostic criteria are not subject to constant modification, they may serve to inhibit rather than to foster scientific progress.

Recent studies indicate that at least some of these clinical constellations are not accidentally derived but are predetermined by heritable susceptibility factors. ${ }^{10,17-19}$ While much remains to be done, it appears inevitable that more intense study of arthritic subsets identified by genetic markers will lead to better understanding of both the pathogenesis and clinical manifestations of the various arthritic diseases. Neither the clinical subsets nor the genetic markers exist in isolation relative to each other. At this moment it seems to the author that pursuit of new information is most likely to be successful if groups are defined by genetic markers rather than by overlapping clinical characteristics. However, at the time of this writing, adequate information is not available to enable us to do so in this chapter. Thus, with the exception of the HLA-B27 group, we continue to divide patients into the officially recognized onset subtypes: systemic, polyarticular, and pauciarticular.

\section{Laboratory Studies}

The ARA-JRA diagnostic criteria do not include laboratory, histologic, or radiographic items. ${ }^{13,14,16}$ The diagnosis of JRA is made by physical examination. Laboratory studies are performed to help in excluding other dis- 
Table 3.3. Disorders Associated with the Presence of Rheumatoid Factor

Rheumatic diseases: RA, SLE, scleroderma, MCTD, Sjögren's syndrome

Acute viral infections: mononucleosis; hepatitis; others, including recent immunizations

Parasitic infections: malaria, schistosomiasis, etc.

Chronic infections and inflammatory disorders: SBE, Tbc, syphillis, salmonellosis, sarcoid, chronic liver disease, etc.

Immune-complex diseases; Waldenström's hypergammaglobulinemia purpura, cryoglobulinemia

orders that have specific laboratory abnormalities, to aid in identification of those subsets of JRA associated with specific serologic or immunologic markers, and to provide a measure of the extent of the inflammation and its systemic effects (see Table 1.6). It will perhaps surprise the nonpediatric rheumatologist that ESR and GRP are often normal in nonsystemic forms of JRA. ${ }^{20,21}$ Extraordinarily high ferritin levels are found in older-age onset systemic JRA. ${ }^{21 a}$

It is important to remember that rheumatoid factors are not specific for RA (Table 3.3). In childhood, most common causes of a positive test for rheumatoid factor are laboratory error (overly sensitive slide tests) or recent viral illness. The overwhelming majority of cases of childhood arthritis are not associated with the presence of rheumatoid factor, and the diagnosis of RA is always obvious in the subset of childhood arthritis that is characterized by the presence of consistently high titers of rheumatoid factor. ${ }^{22}$ The test for rheumatoid factor is, therefore, not performed to help in the diagnosis of JRA but to identify that subset of children whose disease is most likely to resemble early onset severe adult RA. Tests for rheumatoid factor are also commonly positive in children with SLE and in both localized scleroderma and progressive systemic sclerosis.

Antinuclear antibodies (ANAs) are present in about $60 \%$ of arthritic children, with highest frequency in little girls with pauciarticular disease and big girls with classic adult RA. ${ }^{23}$ Speckled and homogenous patterns predominate but all staining patterns may be seen and all are associated with increased risk for iridocyclitis as initially reported by Schaller. ${ }^{24}$ Antihistone ANAs are common in pauciarticular patients and are found in higher titer in those with a history of iritis. ${ }^{25}$

Many other antibodies and immunologic abnormalities have been described in JRA but their significance in the evolution of the disease remains obscure. ${ }^{26-35}$

Synovial fluid is examined to exclude bacterial infection in monarticular disease at its onset or in polyarticular disease in which septic infection seems a diagnostic consideration ${ }^{36,37}$ (see Chapter 2 under Acute and Chronic Infections of the Bones and Joints). Synovial biopsy is obtained only if the tuberculin test is positive. Needle biopsy of the knee in children has been a 


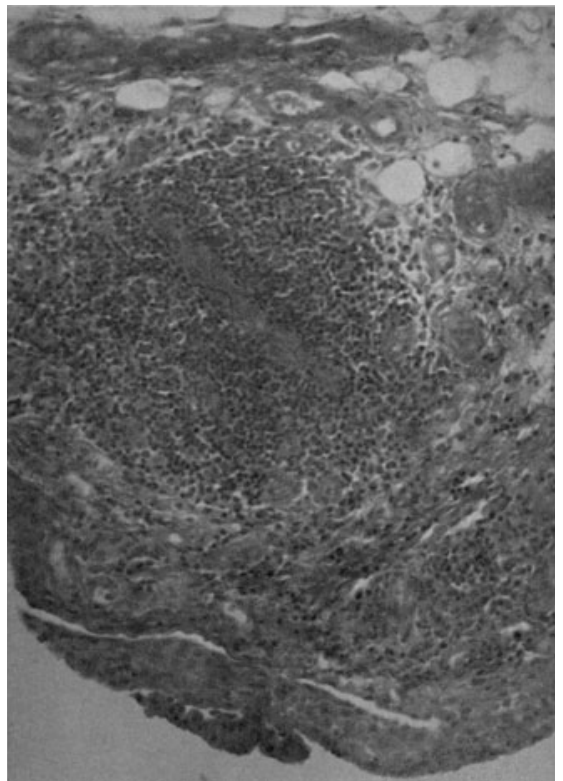

A

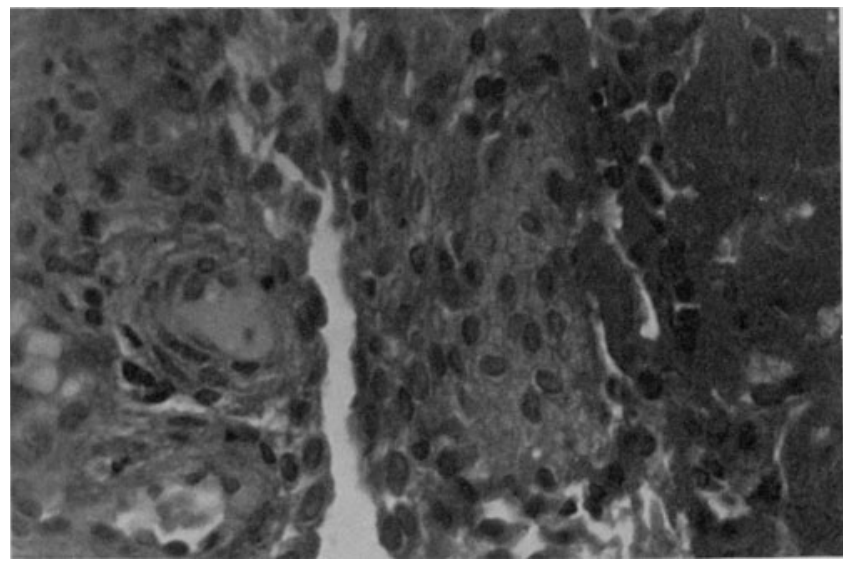

C

Figure 3.1. Three patterns of rheumatoid synovitis in one needle biopsy. (A) A lymphocytic (Allison-Ghormley) nodule in the center field is surrounded by a more diffuse infiltrate of "round" (plasma) cells. (B) Necrotic villi (top left); hypervascular sublining tissues with a diffuse "round cell" (lymphocytic and plasma cell) infiltrate. (C) Fibrinoid necrosis (right margin) and hypertrophic synovial histiocytes lining villi. (A and B) $\times 200 ;(\mathbf{C}) \times 640$. (Reprinted with permission from Jacobs et al., ref 38. Copyright by the American Academy of Pediatrics.)

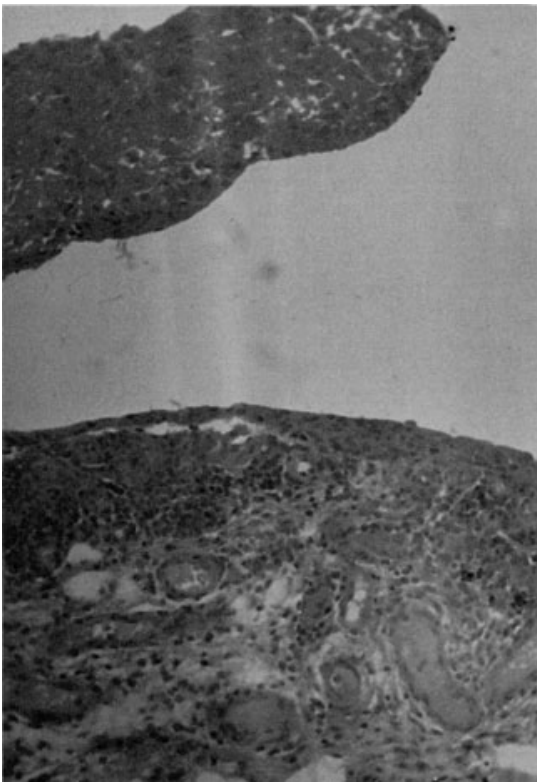

B 


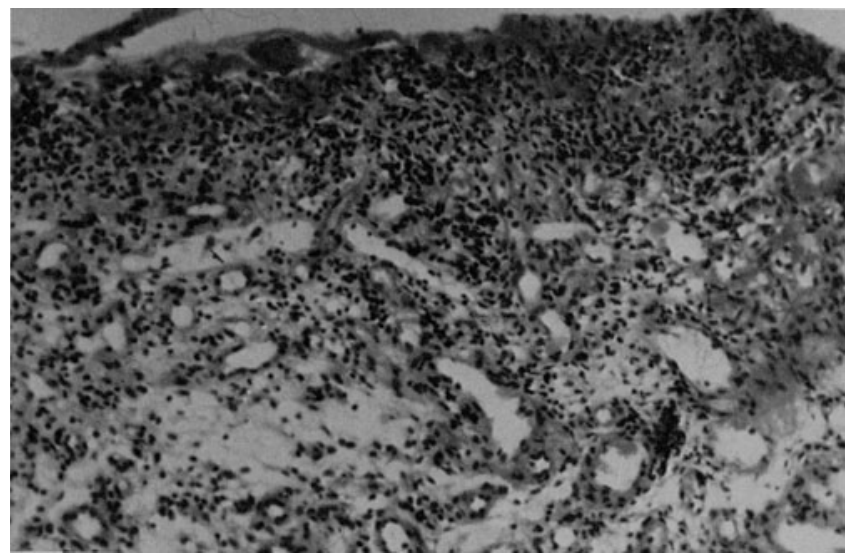

A

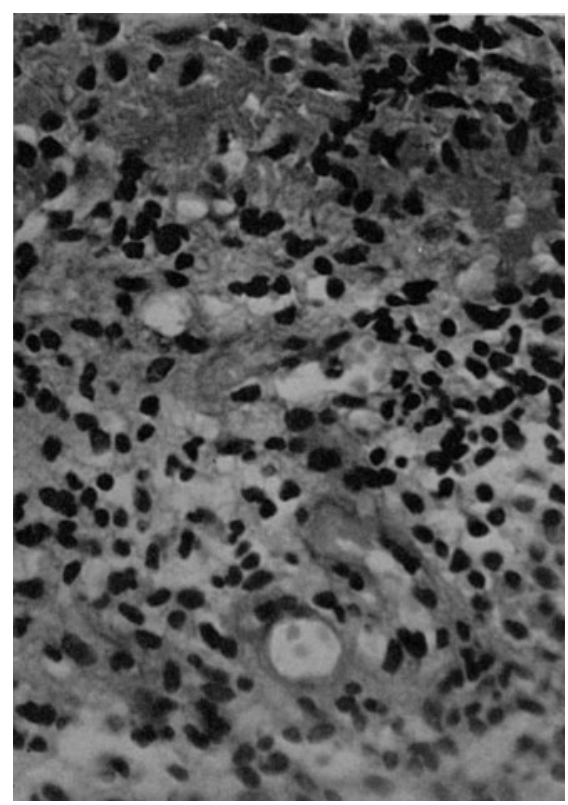

B
Figure 3.2. Rheumatoid synovitis mimicking septic synovitis. (A) The synovial surface is marked by granulation tissue comprising a dense capillary network and a cellular inflammatory exudate. (B) The exudate is largely lymphocytic but interpersed with occasional neutrophils. The surface is lined with necrotic fibrinoid material entrapping reactive cells. (A) $\times 200$; (B) $\times 640$. (Reprinted with permission from Jacobs et al., ref. 38. Copyright by the American Academy of Pediatrics.)

satisfactory technique in our clinic (Fig. 3.1). ${ }^{38}$ However, while biopsy findings may be compatible with RA, the diagnosis cannot be established on the basis of a biopsy (Fig. 3.2). ${ }^{39-41}$ Therefore, pathologic examination is of no value unless it shows a specific nonrheumatoid disease process. While open biopsy should be a harmless procedure, it has generally been followed by immobilization and/or disuse of the joint by the child, with resultant frequent permanent severe loss of function. The yield from open biopsies is so small and the risk so high that we have essentially abandoned doing them except in most unusual cases. ${ }^{38,42}$ Arthroscopic biopsy can be performed if needle biopsy fails, but it has a higher complication rate. 


\section{Radiographic Studies}

There are no radiographic findings that are characteristic of early JRA. Periarticular demineralization, bandlike areas of metaphyseal rarefaction identical to those of leukemia, and dense "growth-arrest" lines are all nonspecific findings that cannot be interpreted as diagnostic of JRA. ${ }^{42,43}$ Periosteal new-bone formation, especially in the fingers, described as an early finding in JRA, is rarely seen in our clinic. We suspect many previously reported cases really represented spondyloarthritis with sausage digits.

Radiographs, ultrasound, CT, bone and gallium scans, and MRI are obtained solely to exclude other disorders that may be confused with JRA. Rarely, a bone scan performed for possible osteomyelitis shows other subclinically involved joints and suggests the diagnosis of childhood arthritis. The diagnosis of JRA is usually easily established long before the changes of chronic arthritis are radiographically apparent. ${ }^{43}$ Although MRI is the most sensitive technique for detecting subtle synovial and cartilage changes, its use in JRA is limited by motion in small children, inability to examine multiple joints, and expense. ${ }^{44-47}$

\section{Epidemiology of Arthritis in Childhood}

Arthritis is not rare in pediatric practice: in a year, one in 100 children will have joint pain requiring evaluation, and about one in 10,000 will develop chronic arthritis. ${ }^{48,49}$ Although studies of childhood arthritis, using differing survey methods and diagnostic criteria, have shown varying prevalence, ${ }^{50} \mathrm{a}$ study from upper New York State showing a prevalence of 1.1 per 1000 in school-age children is probably the most accurate published estimate. ${ }^{51}$ Minor transient musculoskeletal complaints of rheumatic origin are probably even more frequent.

Studies of the age of onset of childhood arthritis all show a peak incidence in the second year of life and a bimodal curve with a second rise later in the first decade. ${ }^{42,50,52}$ Recent studies show that these curves are an amalgam of the varying age-incidence patterns of specific subsets; rheumatoid-factorpositive early onset adult RA and HLA-B27-associated spondyloarthritis contribute to the late-onset peak; the other subsets all have an unusually high incidence between 1 and 4 years of age (Table 3.2)..$^{5-9,50}$

More females than males have arthritis at any age. Females are more frequently clinically affected in all subsets other than the systemic (equal) and HLA-B27-associated (males 2:1). Extrapolation of data from several studies suggests that the early onset pauciarticular and polyarticular groups may have as high as 7:1 female preponderance (Table 3.2). ${ }^{5,8,42}$

The relative incidence of the various-onset subsets of JRA depends on the referral patterns to the reporting clinic. In our own clinic, at present, the overwhelming majority of new patients have pauciarticular disease (Table 
Table 3.4. Mode of Onset of Juvenile Rheumatoid Arthritis

\begin{tabular}{|c|c|c|c|c|c|}
\hline Author & Years & $\begin{array}{l}\text { Number } \\
\text { of Cases }\end{array}$ & $\begin{array}{c}\text { Systemic } \\
(\%)\end{array}$ & $\begin{array}{c}\text { Polyarticular } \\
(\%)\end{array}$ & $\begin{array}{c}\text { Pauciarticular } \\
(\%)\end{array}$ \\
\hline Calabro et al. ${ }^{79}$ & $1960-1977$ & 200 & 20 & 49 & 31 \\
\hline Stillman and Barry 6 & $1923-1977$ & 204 & 19 & 41 & 40 \\
\hline Schaller $^{5}$ & $1967-1977$ & 112 & 20 & 30 & 50 \\
\hline Fink $^{7}$ & $1970-1977$ & 151 & 23 & 18 & 59 \\
\hline Hanson et al. ${ }^{8}$ & $1955-1977$ & 563 & 43 & 23 & 34 \\
\hline Levinson et al. ${ }^{9}$ & $1958-1977$ & 156 & 20 & 33 & 47 \\
\hline Jacobs & $1978-1981$ & 260 & 9 & 16 & 75 \\
\hline
\end{tabular}

3.4). In older-reported series, polyarticular disease was the most common subset. ${ }^{53}$

\section{Familial Incidence}

Siblings are at increased risk - the answer to a question frequently asked by parents-but the exact additional risk is unknown. ${ }^{54}$ The risk in families with affected individuals carrying HLA-B27 or HLA-DR4 markers is not inconsequential. Our experience and that in the literature suggest that in every 350 cases (after exclusion of spondyloarthritis and seropositive patients) one sibling pair will be observed. Since the prevalence of childhood arthritis is thought to be 1:1000 children, the risk to siblings appears to be two or three times higher than would be expected. The familial risk is further enhanced by the presence of iritis and HLA-DR5 ${ }^{54}$ and DR8. ${ }^{55}$ Most parents find this risk inconsequential, all the more so since almost all familial cases are pauciarticular.

Nevertheless, there is evidence that parents of young-onset JRA patients have fewer subsequent children, suggesting some voluntary limiting of fertility. ${ }^{56}$ While there seem to be inherited predispositions to at least some forms of childhood arthritis, the genetic effect is complex (polygenic) and weakly penetrant. ${ }^{57,58}$

\section{Systemic JRA (Still's Disease)}

Children with this form of JRA usually begin their illness with high spiking fever $\left(>103^{\circ} \mathrm{F}\right.$ daily), a typical rash, lymphadenopathy, hepatosplenomegaly, abdominal pain, myalgia, and arthralgia (Fig. 3.3). Sometimes pleurisy, pericarditis, and myocarditis are the predominant manifestations. Arthritis is often relatively inapparent at the onset of the illness. ${ }^{59}$ Uveitis is uncommon in this form of JRA but does occur occasionally. The first clear descrip- 


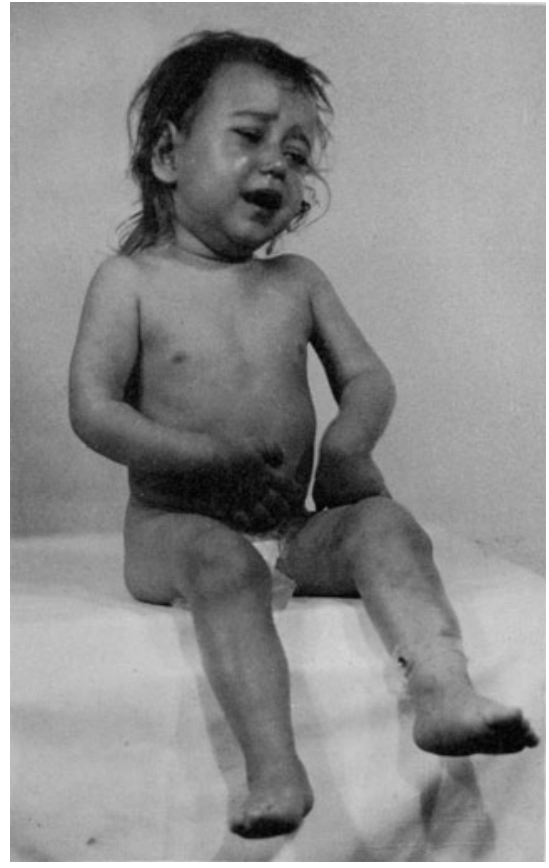

Figure 3.3. Systemic JRA that had its onset at age 5 months.

tion of this form of JRA was by Still in 1896. ${ }^{11}$ Systemic-onset disease accounts for $9 \%$ of new childhood arthritis patients registered in our clinic (Table 3.4).

\section{Differential Diagnosis}

JRA is an important consideration in the differential diagnosis of unexplained fever. However, popularization of the notion of JRA as a cause of most unexplained fevers in childhood has unfortunately resulted in considerable diagnostic error. Physicians sometimes forget that a definite diagnosis of JRA requires arthritis, persistent for at least 6 weeks, plus exclusion of all other disorders that could conceivably cause the fever and arthritis. ${ }^{13}$ Experienced pediatric rheumatologists are well aware that many children for whom this diagnosis is considered on the basis of fever alone will have some other disorder (Table 3.5).60-62 The differential diagnosis of arthritis in childhood is discussed in Chapter 2 and in the chapters on the other specific connectivetissue syndromes. However, the three groups of disorders most frequently mistaken for systemic JRA are infection, inflammatory bowel disease, and malignancy; these entities account for $50-73 \%$ of children with prolonged "fever of unknown origin." The infections tend to be osteomyelitis or hidden 
Table 3.5. Causes of Prolonged Fever of Unknown Origin in Childhood

\begin{tabular}{|c|c|c|c|c|c|}
\hline \multirow[b]{2}{*}{ Ultimate Diagnosis (\%) } & \multirow[b]{2}{*}{ McClung ${ }^{62}$} & \multirow[b]{2}{*}{$\begin{array}{l}\text { Lahr and } \\
\text { Hendley }\end{array}$} & \multicolumn{2}{|c|}{ Pizzo et al. ${ }^{60}$} & \multirow[b]{2}{*}{ Adults* } \\
\hline & & & $\begin{array}{l}\text { Under } \\
\text { Age } 6\end{array}$ & $\begin{array}{l}\text { Over } \\
\text { Age } 6\end{array}$ & \\
\hline Infection & 29 & 33 & 65 & 40 & $31 / 23$ \\
\hline JRA & 6 & 13 & 6 & 15 & $4 / 3$ \\
\hline $\begin{array}{l}\text { Inflammatory bowel } \\
\text { disease }\end{array}$ & 3 & 6 & & 9 & $2 / 2$ \\
\hline Malignancy & 8 & 13 & 8 & 4 & $31 / 7$ \\
\hline SLE & 3 & & & 6 & \\
\hline $\begin{array}{l}\text { Familial Mediterranean } \\
\text { fever }\end{array}$ & 4 & & & & $1 / 1$ \\
\hline $\begin{array}{l}\text { Factitious fever (parent- or } \\
\text { child-induced) }\end{array}$ & 9 & 4 & & & $3 / 4$ \\
\hline Other causes ${ }^{* *}$ & 17 & 12 & 15 & 6 & $16 / 34$ \\
\hline Never diagnosed & 21 & 19 & 6 & 20 & $12 / 26$ \\
\hline
\end{tabular}

*Adult data from Larson, Medicine 61:269-292, 1982 and Knockaert, Arch Intern Med 152:51-55, 1992.

** Includes anhidrotic ectodermal dysplasia. ${ }^{64}$

abscess, most often in the abdomen or pelvis. The most common malignancies are leukemia, lymphomas, and neuroblastoma. Weight loss, fever, and arthralgia are common presenting manifestations of these malignant disorders. ${ }^{63}$ Bone pain out of proportion to visible arthritis or accompanied by refusal to walk suggests malignancy rather than JRA. Even after exclusion of malignancy and infection, only $10-20 \%$ of children with unexplained fever turn out to have JRA (Table 3.5).

There is no way to avoid an extensive workup for children who are thought to have systemic JRA but who do not fulfill the diagnostic criteria. As we have emphasized, even in those with arthritis, other causes must be excluded. For those without obvious arthritis, a presumptive diagnosis of JRA may be made only after the most thorough study excludes the common mimics. Such a workup routinely includes chest and skeletal radiographic surveys, abdominal ultrasound, technetium bone scan, gallium scan, bone-marrow examination, and, when appropriate, a small-bowel series, colonoscopy, barium enema, and CT and MRI scans. ${ }^{65}$ If lymphadenopathy is present, a lymph node biopsy may also be necessary. ${ }^{66}$ The entire workup can be completed in a few days. If all of these studies are normal, a child with hectic fever, in the absence of arthritis, may be considered to probably have JRA and may be managed as if he does. The diagnosis, however, remains tentative, and it is appropriate to remain alert to missed clues to other diagnostic possibilities. 
Table 3.6. Manifestations of Systemic JRA

Hectic fever

Salmon-pink evanescent rash

Arthritis and torticollis

Myalgia

Hepatosplenomegaly and Iymphadenopathy

Tenosynovitis

Pericarditis and myocarditis

Pleurisy and lung infiltrates

Abdominal pain

Irritability, drowsiness, meningismus

Acute laryngeal stridor

Anemia, leukocytosis, thrombocytosis, greatly elevated ESR and interleukin-677

\section{Epidemiology}

The most common age at onset is between the first and fourth birthday. ${ }^{42,50,67,68}$ However, both adults and children at any age may be affected. ${ }^{15,69-72}$ Our youngest patient was 6 weeks old. Up to one-third of cases begin in teenagers or adults. ${ }^{4,50}$ Males and females are equally frequently affected. Onset is uncommon in winter. ${ }^{73}$

\section{Manifestations (Table 3.6)}

Fever. A typical fever pattern has been described (quotidian) as one or two daily hectic spikes, sometimes to $105^{\circ} \mathrm{F}$, and then a return to $98.6^{\circ} \mathrm{F}$ or below. ${ }^{59,74}$ Our experience suggests that return to subnormal temperature $\left(<98^{\circ} \mathrm{F}\right)$ usually occurs only in those given aspirin or NSAIDs at the time of the peak. ${ }^{74}$ Children given no medication at the height of the spike tend to have hectic fever without return to below normal (Fig. 3.4). The fever spikes often take place late in the afternoon or in early evening and may be accompanied by shaking chills. Children who do not have pleurisy, pericarditis, or

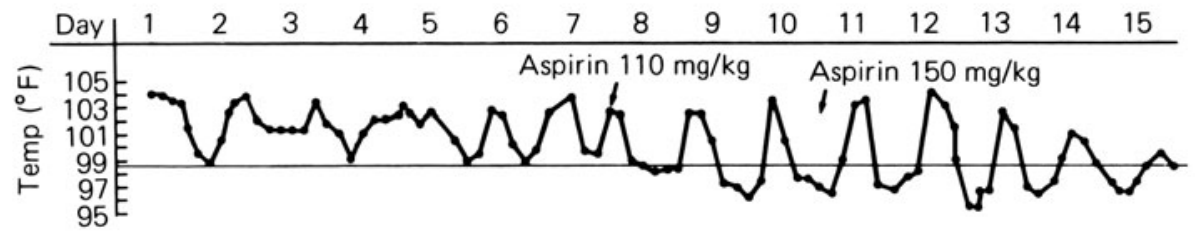

Figure 3.4. Typical hectic fever pattern of systemic JRA (patient pictured in Fig. 3.5). The temperature does not generally fall below $98.6^{\circ}$ unless aspirin is given, as shown during the week of observation without therapy. Some response is noted soon after aspirin is begun, but satisfactory control of fever is dependent on achieving a therapeutic level of salicylate. Steady-state salicylate levels are not achieved until about the ninth day of therapy. 


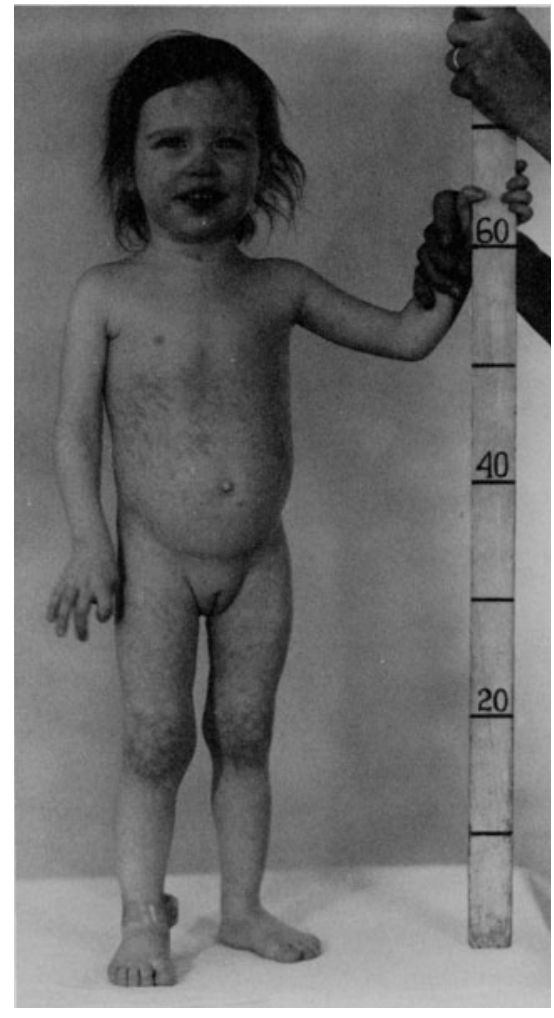

Figure 3.5. Typical rheumatoid rash in 2 year-old dwarfed child who had a year of fever and rash before arthritis was apparent. The Koebner phenomenon is apparent where she has scratched the abdomen. On the thighs, the rash is associated with a livedo reticularis pattern.

myocarditis often look surprisingly well at the time they are febrile as compared to children febrile with acute disorders.

Rash. A distinctive evanescent salmon-pink macular or maculopapular rash, most commonly on the trunk or overlying joints, occurs in most children with systemic JRA (Fig. 3.5)..$^{75,76}$ As one looks at the rash, it seems to change slightly, with new spots appearing while others disappear. Rubbing or scratching the skin may elicit the rash (Koebner phenomenon); in about $25 \%$ of cases, the rash itches. If one is sufficiently familiar with the rash, a presumptive diagnosis can be made on the basis of the rash and fever alone. However, occasionally, catecholamine-secreting tumors or other disorders may present with a similar rash. Thus the presence of typical rash does not relieve the physician from the need to exclude other diagnostic possibilities.

The rash of JRA is often most pronounced at the time of the fever spikes. If these are limited to evenings, it may he worthwhile for the physician to reexamine the child at that time, as the fleeting rash may not have been noticed by the family or hospital staff. The rash of JRA may come and go during the course of treatment even though fever and arthritis are controlled with medication. 
Biopsy of the rash is not necessary since it is the clinical characteristics rather than the pathologic features that are useful to the physician. Where biopsies have been performed for academic study, the primary finding has been edema, as in urticaria. In florid cases, mild perivascular infiltrates of lymphocytes or polymorphonuclear cells may be seen in the loose connective tissue of the subepithelial layer. ${ }^{76,78}$ When fever and arthritis are accompanied by a rash suggesting impetigo or psoriasis, Reiter's syndrome is the most likely diagnosis (see Chapter 4).

Arthritis. Children with systemic JRA fall into two groups: (1) those with obvious severe, unremitting polyarticular disease who have a rather poor prognosis for ultimate joint function and who account for the major crippling and death in JRA and (2) those with arthralgia without much arthritis who have an excellent prognosis for future joint function and often must represent a different disorder from the severe polyarticular systemics. ${ }^{42,79,79 a}$ While the severe polyarticular systemic-onset JRA population represents a discouraging challenge to the pediatric rheumatologist and a fearful prospect to parents of children diagnosed as JRA, it constitutes a very small percentage of children with arthritis, perhaps $2 \%$ of those registered in our consultation clinic.

Torticollis. During the acute attack, more than half the children have neck pain and torticollis. Neck pain is often the most prominent arthritic manifestation in systemic-onset patients. ${ }^{6}$ Cervical-spine pain, therefore, may be a good clue to diagnosis in children with relatively little visible arthritis. On the other hand, patients with torticollis have been referred to us who had eosinophilic granuloma, craniopharyngioma, and unrecognized rotatory cervical subluxations; ${ }^{80}$ osteomyelitis of a first rib can also cause torticollis. ${ }^{81}$

Myalgia. Myalgia is often a prominent symptom in Still's disease, especially in children with little arthritis. ${ }^{82}$ Elevations of creatinine phosphokinase are generally mild, if present at all. ${ }^{83}$ The severity and quality of the muscle pain in systemic JRA resemble the degree seen in adult polymyalgia rheumatica and this presumably is a manifestation of vasculitis in muscle. Lumbar, cervical, and thigh muscles are most commonly affected. Muscle weakness is not seen. Muscle biopsies are obtained only in children thought to have polyarteritis nodosa or some other form of systemic vasculitis. In Still's disease, biopsies have usually been normal, but occasionally a perivascular accumulation of round cells has been found in connective tissues surrounding muscle, especially in adults. ${ }^{69,84}$

Hepatosplenomegaly. Systemic JRA is frequently accompanied by enlargement of the liver and spleen. Minor abnormalities in SGOT and SGPT are common. ${ }^{83}$ Sometimes the hepatomegaly is alarming, extending $10 \mathrm{~cm}$ below the costal margin. When liver biopsies have been obtained, only nonspecific inflammation in the periportal areas has been seen. ${ }^{85}$ 


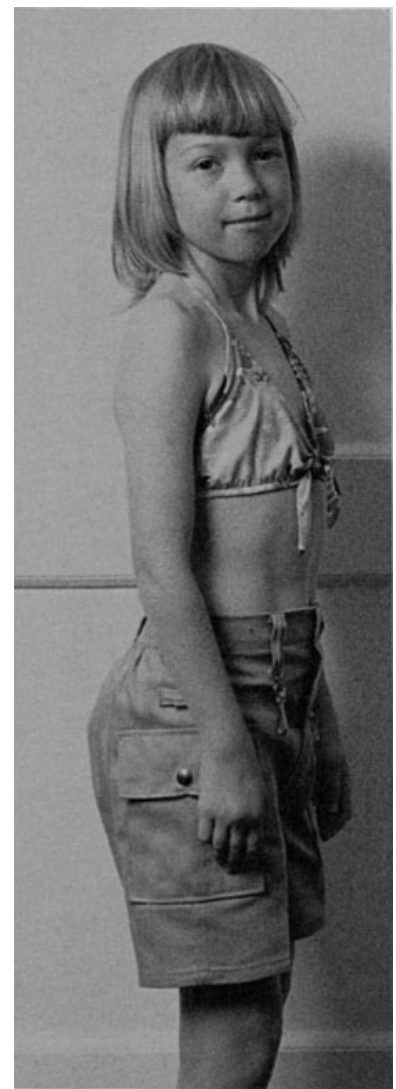

Figure 3.6. Proliferative polyarticular JRA with cystic "synovial pouches" at the wrists and shoulders. Stance indicates flexion contracture at the elbow with limited supination, often an early but unrecognized arthritic manifestation in children. The accentuated lordotic posture is a clue to disease in the hips and is accommodated by standing with the knee bent. Some of these girls do not complain of pain (as initially observed by Still ${ }^{11}$ and emphasized by Grokoest et al. ${ }^{42}$ ), and an early diagnosis may not be made at the time of routine pediatric examiniation unless the joints are carefully examined.

Lympadenopathy. Nondiagnostic lymphoid reactive follicular hyperplasia is also most always present in systemic JRA: at times, the nontender and freely movable lymph nodes may reach $5 \mathrm{~cm}$ in diameter. ${ }^{86}$ Nuclear debris with phagocytosis by histiocytes (necrotizing lymphadenitis) is seen occasionally. ${ }^{87}$

Tenosynovitis. Proliferative inflammation about the wrist commonly creates dorsal synovial pouches (Fig. 3.6). Sometimes these "masses" are mistaken for "tumors." Synovial pouches are also occasionally seen in other joints (Fig. 3.7). An unusual manifestation of tenosynovitis that we have seen in two children with systemic JRA is tenovaginitis of the superior oblique tendon of the eye, resulting in Brown's syndrome. This syndrome is characterized by intermittent vertical diplopia and a clicking sensation on trying to move the eye up and inward; the click is followed by resumption of normal vision. This syndrome has also been reported in adults with arthritis. ${ }^{88}$

Pericarditis and Myocarditis. About one-half of children with systemic JRA have pericarditis during the initial attack often with pleuritis and sometimes 


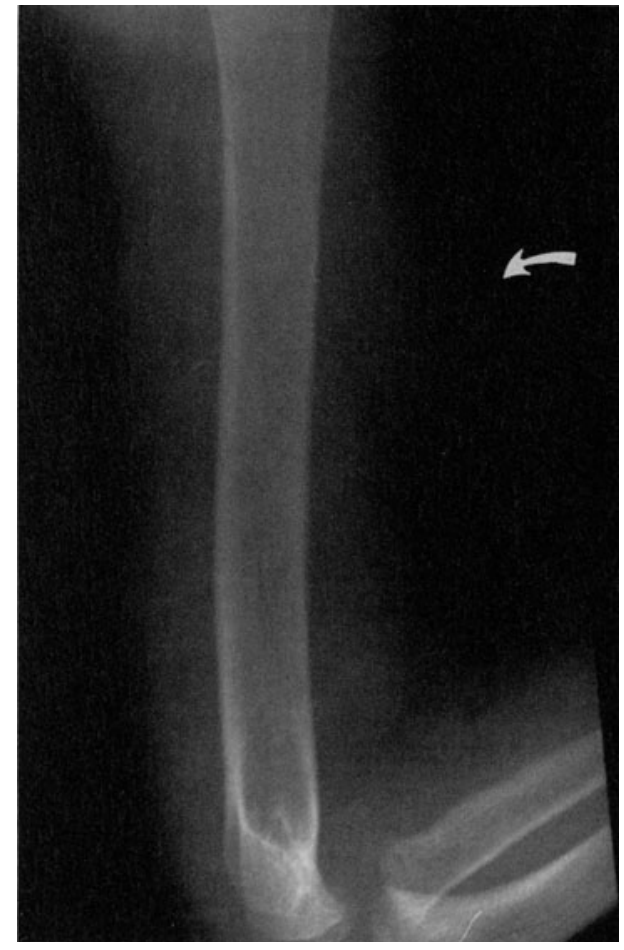

Figure 3.7. Shoulder effusion dissecting into biceps muscle creating a synovial cyst seen on plain radiograph $(\mathbf{A})$ where it was thought to represent biceps tendon rupture. Ultrasound (B) indicated a cyst which disappeared spontaneously. Patient had same problem on opposite side on another occasion. Similar cystic dissections may be seen below the knee in the gastrocnemius muscle.

A

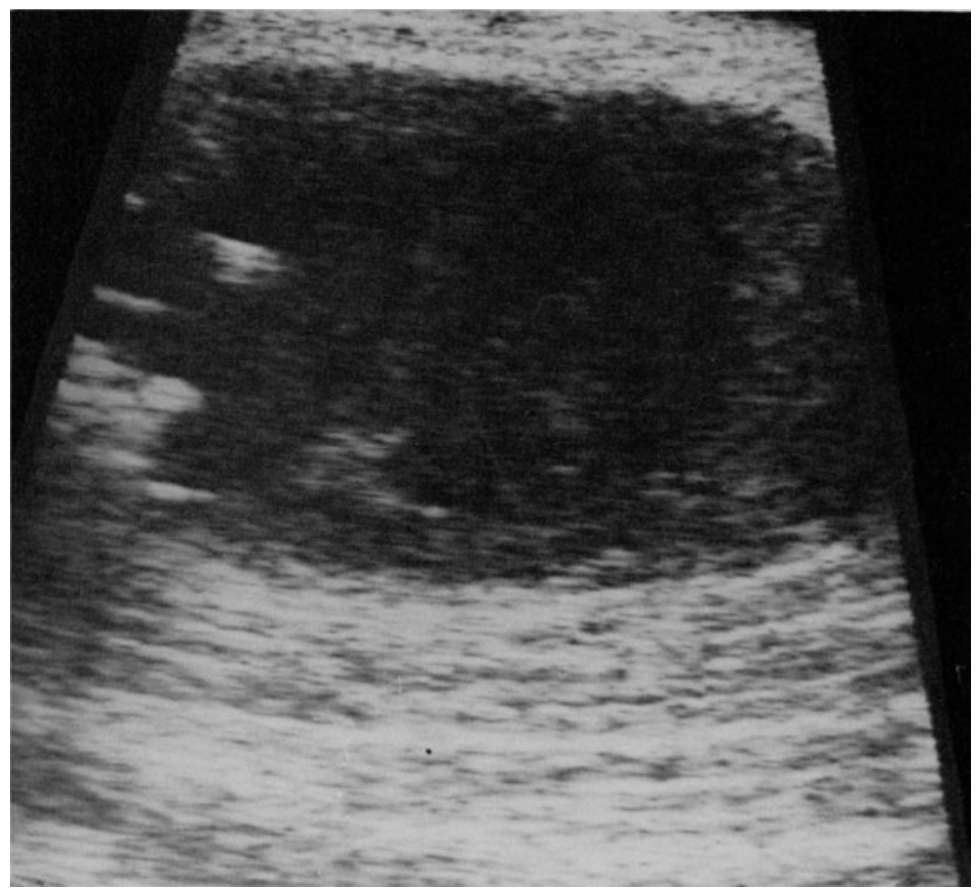


a triad including pneumonitis. ${ }^{89}$ Usually, the pericarditis is asymptomatic or manifested only by minimal chest pain, and diagnosis depends on the demonstration of echocardiographic, radiographic, and electrocardiographic abnormalities (Fig. 3.8). Occasionally, the pericarditis is associated with myocarditis, cardiac tamponade, and severe congestive heart failure (Fig. 3.9) ${ }^{90-92}$ Attacks of pericarditis and myocarditis usually last between 1 and 15 weeks, with an average of 2 months. However, some patients tend to have recurrent attacks. ${ }^{93-96}$ These patients resemble patients with recurrent Coxsackie B viral pericarditis and myocarditis. ${ }^{95,96}$ In one adult with this syndrome, Coxsackie B3 virus could still be demonstrated in the pericardial fluid 1 1/2 years after the first of many recurrent episodes. ${ }^{97}$ Adenovirus has also been demonstrated in the pericardial fluid of one child at the onset of systemic JRA, ${ }^{98}$ and we are aware of adenovirus isolation from bone marrow and pleural fluid in two other patients at the onset of systemic JRA. A similar vasculitis syndrome has also been associated with the demonstration of high titers of hepatitis $B$ virus antigen in pericardial fluid. 99 Two cases of hemophagocytic syndrome, characterized by systemic proliferation of benign hemophagocytic histiocytes, resulting in fever, hepatosplenomegaly, and cytopenia, ${ }^{100}$ suggesting viral infection at the time of onset of systemic JRA have been reported, ${ }^{101,102}$ and elevations of antibody titers to Coxsackie virus have occasionally been noted. ${ }^{103,104}$ An immunodeficient individual has had arthritis as a manifestation of persistent adenovirus infection in the synovium, analagous to the dermatomyositislike syndrome seen in agammaglobulinemic children with persistent viral infection of the central nervous system. ${ }^{105}$ These are isolated cases, however, and in most patients with pericarditis the pathogenesis of the episode is unknown and there is little to support a viral etiology for JRA. ${ }^{106}$

Although symptomatic constrictive pericarditis has not been reported in childhood JRA, adhesive pericarditis is a common finding in the select population of children with JRA who are examined postmortem.11,93,94 Myocarditis that was not recognized during life may be demonstrated at postmortem examination (Figs. 3.8 and 3.9).

Valvulitis. Rheumatoid nodules on the heart valves and chordae tendineae have not been reported in children, but we have seen one child with seropositive RA associated with tuberculosis in whom rheumatoid nodules were demonstrated in the lung, heart, and meninges at the time of postmortem examination (Fig. 3.10). These findings were analogous to those seen in the animal model of arthritis called "adjuvant arthritis". Aortic insufficiency occasionally occurs in polyarticular disease. ${ }^{107}$

Pleurisy and Lung Infiltrates. One-third of children with systemic-onset JRA have radiographic evidence of pleural effusions during the acute episode. These are often associated with pericarditis and are sometimes also accompanied by transient lung infiltrates that disappear along with the pericarditis and pleurisy as the disease comes under control. ${ }^{4}$ Still's disease is an 


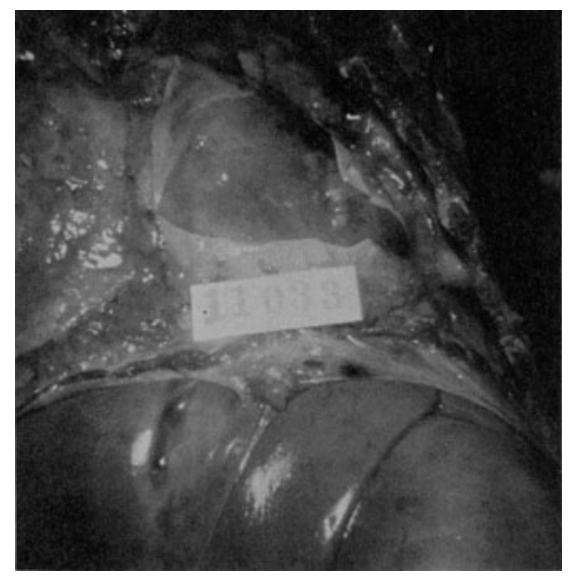

Figure 3.8. Fibrinous pericarditis was found at autopsy in a child with systemic JRA who died following the second injection of gold (see Fig. 3.43). The pericarditis was clinically silent and unrecognized.

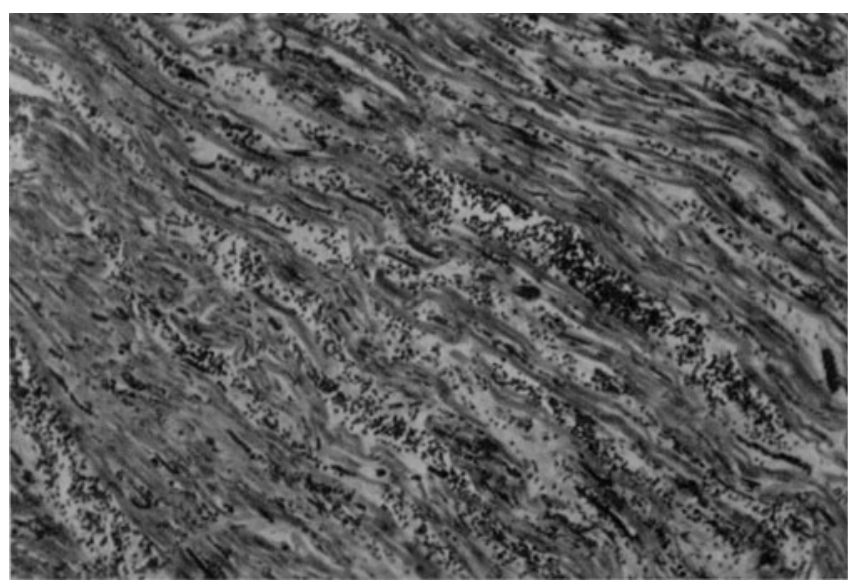

Figure 3.9. Flagrant myocarditis noted in the same patient (Fig. 3.8) was also "silent."

important consideration in the differential diagnosis of the syndrome of recurrent pleurisy and pericarditis in childhood. Occasional cases of fibrosing alveolitis, lymphoid interstitial pneumonia, rheumatoid nodules in the lung, and recurrent episodes of pulmonary hemorrhage have been seen in children with arthritis. ${ }^{108-111}$ Subclinical abnormalities of pulmonary function may be common in JRA. ${ }^{112}$ One case of primary pulmonary hypertension has been reported. ${ }^{113}$

Abdominal Pain. Diffuse abdominal pain suggestive of serositis is a prominent symptom in about $10 \%$ of children with systemic JRA. ${ }^{86}$

Laryngeal Stridor. Systemic JRA occasionally presents with stridor as the most apparent symptom, a result of life-threatening acute cricoarytenoid 


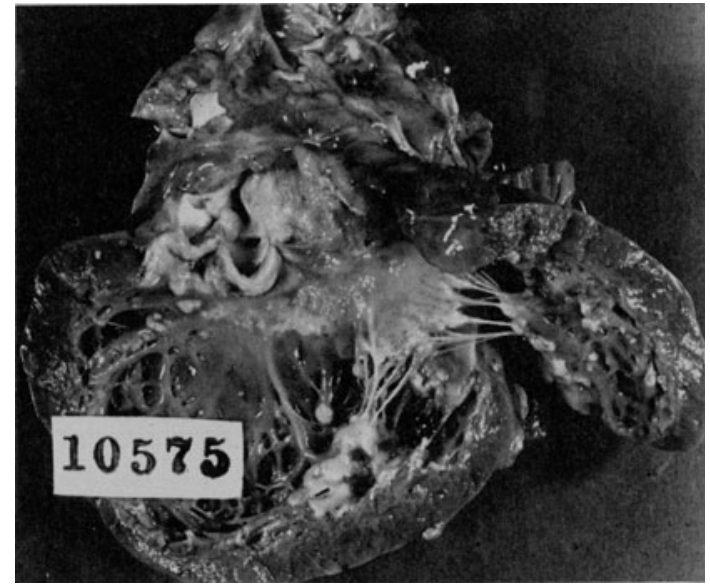

the role of microbial superantigens and heat shock proteins in the creation of such a disease process, and of receptor-targeted immunotherapy (See: Bull Rheum Dis 41\#3, 1992).

Figure 3.10. Endocardial rheumatoid nodules found at autopsy in a 6-year-old girl with seropositive JRA who died of congestive heart failure. Left ventricle, mitral, and aortic valve granulomas caused stiff valves and extended to the chordae tendineae and the endocardium of the papillary muscles, trabeculae carneae, and adjacent ventricular endocardium. Patient also had arrested tuberculosis. Today one could study

arthritis. ${ }^{78,114}$ Although arthritis may also be demonstrable elsewhere, it is usually not noted in the context of caring for the child with a compromised airway.

Cerebral Manifestations. Irritability, drowsiness, meningismus, and nonspecific electroencephalographic changes are all frequently observed in systemic JRA. ${ }^{115}$ An episode of cerebral vasculitis has been documented by cerebral angiogram in one child with JRA. ${ }^{116}$ O'Connor reported one child with hemiparesis, and we have had one patient with a transient hemiparesis. ${ }^{117}$ Occasional children have been reported with either febrile or afebrile seizures. Perivascular mononuclear-cell inflitrates in the brain and mononuclear inflammation of the meninges were demonstrated in all patients whose brains were examined in a postmortem study. ${ }^{117}$ Immune complexes were found in the spinal fluid of one of those children. Central nervous system (CNS) involvement is probably more frequent than has been previously recognized in Still's disease. However, it is not as frequent as suggested by postmortem material, which reflects the incidence only in the most severely ill patients at the time of death.

Renal Disease. Significant renal pathology is not a part of JRA except in those few children who develop amyloidosis. However, transient albuminuria, leukocyturia, erythrocyturia, hypercalcuria, and decreased creatinine clearance are reported to occur in about one-third of especially carefully followed patients if repeated examinations were performed looking for these abnormalities. ${ }^{118-120}$ Minor changes may also be seen on renal-biopsy specimens. ${ }^{118}$ While some of the renal findings must be a result of damage by drugs $^{121}$ (see below); it also seems likely that some may be a manifestation of 
the disease itself, albeit generally a manifestation of seemingly little consequence. ${ }^{122}$

Amyloidosis. Amyloidosis may be a complication of any chronic disease and in the past accounted for up to $50 \%$ of deaths from childhood arthritis. ${ }^{42,123,124,125}$ Transient proteinuria is the first manifestation, but the diagnosis is usually not suspected until more persistent proteinuria occurs. Associated symptoms include nephrotic edema, hypertension, hepatosplenomegaly, abdominal pain, diarrhea, and congestive heart failure. Diagnosis is usually achieved by aspiration of abdominal fat, ${ }^{126}$ but scintigraphy with 123I serum amyloid $\mathrm{P}$ component may be the best way of diagnosing, locating, and monitoring the extent of systemic amyloidosis. ${ }^{127,127 a}$ Amyloidosis is very rare in children in the United States; the reason for the increased incidence in European pediatric rheumatology clinics is unknown but may be related to the tendency of such clinics to serve disproportionate numbers of severely affected and immunologically deficient individuals. ${ }^{128}$

Amyloidosis has been reported in equal numbers of male and female arthritic children and is not limited to those with systemic disease. ${ }^{121,124}$ One might suspect from this observation that there is at least equal risk of amyloidosis in severely affected childhood-onset male spondyloarthritis, although data to confirm such a hypothesis are not available at present. Colchicine may prevent amyloidosis or cause it to regress. ${ }^{129}$

Renal Papillary Necrosis. Renal papillary necrosis (RPN), a known complication of analgesic use, has not been associated with the use of aspirin alone in JRA. ${ }^{130}$ Wortmann et al. reported RPN in three severe JRA patients treated with many drugs. ${ }^{131}$ The children had unexplained hypertension or hematuria; IVPs demonstrated filling of an entire papillary region, typical of RPN. The authors emphasized that all of their patients were chronically dehydrated, which may cause RPN and which would certainly increase the risk of RPN with anti-inflammatory drug therapy. Care should be taken to avoid chronic dehydration in children receiving analgesic therapy. However, Allen et al. recently observed RPN in five children treated with NSAID who had only pauciarticular disease. ${ }^{132}$

Anemia. Hemoglobin of less than 10 grams (g) has been reported to occur in $39 \%$ of children with JRA at some time during their course. ${ }^{86}$ The anemia seems to be a result of a combination of factors, including iron deficiency ${ }^{133}$ (poor diet, increased losses as a result of medications, malabsorption of oral iron, ${ }^{134,135}$ impaired release of iron stores), shortened life-span of red blood cells, impaired release of erythrocytes from a hyperplastic bone marrow, ${ }^{86}$ blunted erythropoietin production, ${ }^{136}$ and interleukin I-mediated serum suppression of erythropoeisis. ${ }^{137,138}$ Erythroid hypoplasia has also been reported in one child, ${ }^{139}$ and we have seen one child with generalized marrow hypoplasia in our clinic. 


\section{Case Report}

A 3-year-old boy was admitted to the Babies Hospital in April 1972 with a 2-week history of fever and polyarthritis. A diagnosis of JRA was made, and treatment was begun with aspirin with only partial control of his symptoms. His hemoglobin fell to $5.6 \mathrm{~g}$, his WBC to $1400 / \mathrm{mm}^{3}$, and his platelet count to 52,000 . The bone marrow was hypoplastic. His symptoms were controlled with the addition of tiny doses of prednisone ( $3 \mathrm{mg}$ daily) to his aspirin regimen, and the hematologic parameters returned to normal as his symptoms subsided. Four months later, he had an exacerbation of symptoms while receiving a lowered dose of aspirin. Three weeks later, he was readmitted with pancytopenia (hemoglobin, $6.3 \mathrm{~g}$; WBC, 800; platelet count, 32,000). At this time, he was found to have pneumococcal bacteremia. He was treated with appropriate antibiotics, blood and platelet transfusions, and, after control of the infection, with prednisone, $30 \mathrm{mg}$ daily. Aspirin treatment was subsequently reinstituted and prednisone gradually withdrawn without ill effect. After 2 years, his arthritis remitted, and aspirin treatment was discontinued. However, 2 years later, he again developed high spiking fever and polyarthritis. Although the arthritic symptoms responded to aspirin, he again developed pancytopenia with a severely hypoplastic marrow, which again responded to prednisone therapy. Manifestations of systemic JRA persisted for many years, requiring treatment with prednisone as well as many other antirheumatic medications, including choline salicylate. He remained somewhat anemic, but his WBC and platelet count were normal.

Although children with G6PD deficiency are generally instructed not to take salicylates, studies suggest that a therapeutic level of salicylate in G6PD-deficient individuals does not produce hemolysis. ${ }^{140}$

It is often impossible to utilize ordinary laboratory methods to document iron deficiency in children with severe JRA. Serum iron and iron-bindingcapacity determinations are altered by chronic disease. Serum ferritin and transferrin are often elevated in children with active arthritis even if they are iron deficient, and so cannot be used as a guide to iron deficiency. ${ }^{141}$ Bonemarrow stains for iron are a satisfactory method of proving iron deficiency but are impractical except in special circumstances. Although a therapeutic trial of iron therapy is not the ideal method of diagnosing iron deficiency, it is often the only practical approach in children with JRA. ${ }^{133}$ In a recent study of adults with classic RA the combination of low MCV and very high serum ferritin and transferrin always indicated iron deficiency. ${ }^{142}$

Other nutritional anemias have not been documented in JRA. ${ }^{143}$ However, some nutritional anemias may be drug induced, and all deficiency anemias may be intertwined biochemically and pathologically. ${ }^{144}$ Further study of the possible role of deficiencies of vitamins and minerals in these children is needed.

That part of the anemia that is due to iron deficiency generally responds to proper oral iron administration. However, occasionally oral iron is not absorbed and parenteral iron administration is required. ${ }^{134,139}$ We have seen 
no adverse reactions to intramuscular iron but intravenous iron-dextran infusions may exacerbate arthritis. ${ }^{145}$ Severe anemia unresponsive to iron therapy is sometimes a major manifestation of the systemically ill patient. When systemic disease is controlled, anemia often disappears. Occasionally, very ill patients will require alternate-day prednisone or methotrexate therapy to control otherwise-intolerable anemia. It remains to be determined whether recombinant human erythropoeitin is of value. ${ }^{146,146 a}$

Disturbances of Linear Growth (Dwarfing). Somatic growth arrest was reported as an important feature of systemic or severe polyarticular JRA by Still, ${ }^{11}$ and was reemphasized by Kuhns and Swain, ${ }^{147}$ by Coss and Boots (Fig. 3.5), ${ }^{148}$ Ansell and Bywaters, ${ }^{149}$ and Bernstein et al. ${ }^{150}$ Growth-arrest lines in the metaphyses of ill children are a reflection of the cessation of growth due to end-organ inability of the bones to respond to growth hormone and/or a direct inhibitory effect of illness on growing cartilage. Growthhormone deficiency is not a part of JRA, ${ }^{151,152}$ and remission or control of the disease is associated with prompt catch-up growth (Fig. 3.11). ${ }^{149}$ Permanent dwarfing results only from disease that begins early and is uncontrolled throughout childhood or from steroid therapy (Fig. 3.11). ${ }^{149,153}$ We will soon know whether these non-growth-hormone-deficient children can respond to

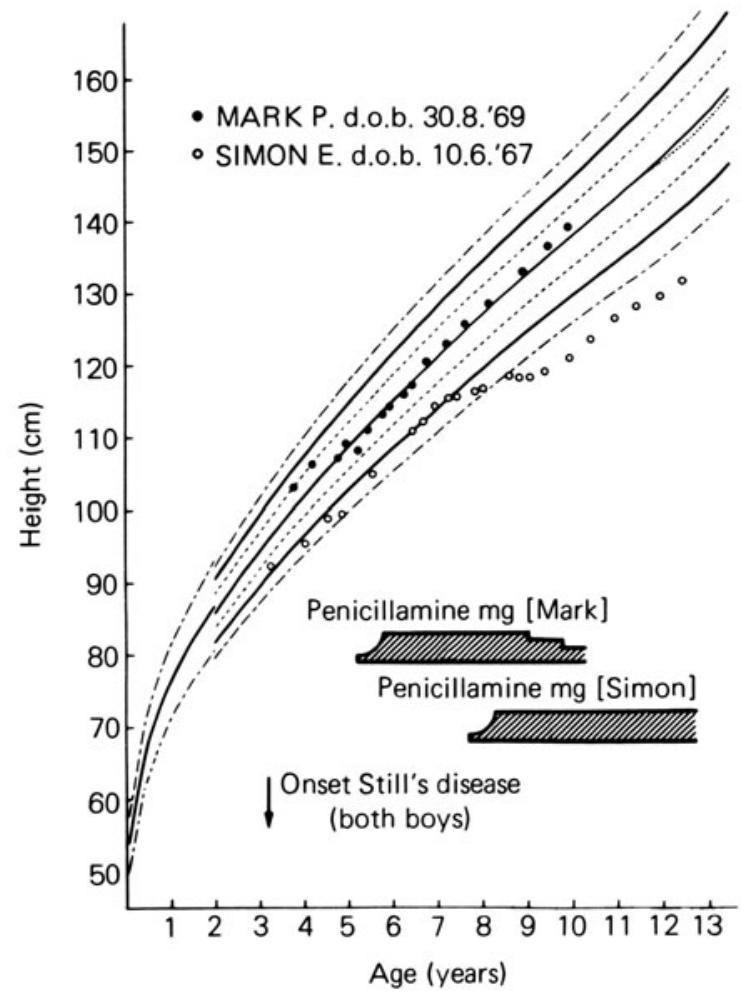

Figure 3.11. Fall-off in linear growth associated with poorly controlled JRA. Patient Mark P. had quick resumption of growth as his disease came under control but will not quite achieve his original anticipated adult height. Patient Simon E., with an inadequate response to drug therapy, will remain dwarfed despite recent control of disease. (Reprinted with permission from Ansell and Hall, ref. 376.) 
exogenous growth hormone and achieve near-normal height. ${ }^{154}$ If so, it will probably require six injections weekly for many years. There may be potential hazards to the growth plates, ${ }^{155}$ and while growth hormone may combat steroid catabolism of protein it enhances the adverse effects on carbohydrate metabolism and increases glomerular filtration rate, which may alter drug excretion, so there my be problems with long-term use in arthritic children. While large daily doses of corticosteroids may result in some irreversible stunting of growth, morning alternate-day steroid or corticotropin therapy may actually foster linear growth if the systemic manifestations associated with growth failure are controlled by a dose of corticosteroids that does not inhibit growth. ${ }^{153,156-158}$ Height may also be adversely affected by collapse of vertebral bodies secondary to steroid therapy and immobility, and by flexion contractures at the hips and knees. ${ }^{149,150}$

Other causes of growth failure, including hypothyroidism and autoimmune polyendocrinopathy, have been reported in association with JRA and must be excluded in arthritic children with growth failure. ${ }^{159-161}$

Ectopic Ossification. Occasionally, children with severe long-standing JRA develop new-bone formation in tissues that do not normally ossify. ${ }^{42,51,162}$ This occurs most often in subcutaneous tissues adjacent to affected large joints or in areas subject to pressure from splints or shoes but has also been noted in the eye, brain, and spinal cord. Spurlike calcifications in ligaments, previously reported as a feature of JRA, were more likely manifestations of spondyloarthritis, and most of these children with ectopic calcification may represent examples of severe spondyloarthritis. ${ }^{162}$

\section{The Course of Still's Disease}

Children with systemic-onset disease separate themselves into two categories. In one group (about $40 \%$ of systemic JRA), there is very little arthritis. Therapy tends to completely control the arthritis, which disappears when the febrile episode subsides. While severe, the illness has a relatively brief course. Febrile episodes rarely last more than 6 months at a time but frequently recur at least once. ${ }^{4,74,79}$ These patients usually ultimately recover without sequelae or with minimal joint dysfunction.

A second group of patients develops arthritis that does not remit when the systemic symptoms are controlled. This includes the $25 \%$ of systemic-onset patients who develop incessant intractable polyarticular disease that is progressively destructive; permanent handicap is inevitable. In most olderpublished series, this group was selectively overrepresented and equaled about $5 \%$ of the total childhood arthritis population. Recent registrations in our clinic indicate that most arthritis in childhood is pauciarticular, and the proportion of these terribly ill, unremitting crippled children has decreased to less than $2 \%$ of all arthritis in children. The remaining $35 \%$ of systemic-onset patients tend to have a spectrum of polyarticular 
disease that may ultimately remit, leaving some scars but a normally functioning adult. Forty percent of patients with recurrent attacks requiring steroid therapy require hip prosthesis in a series followed for an average of 23 years. ${ }^{163}$

\section{Death in Still's Disease}

The incidence of death has progressively decreased from $7 \%$ reported in earlier series ${ }^{42}$ to $5 \%{ }^{164}$ to $2 \% .{ }^{165}$ Since almost all deaths in the United States now are in the systemic polyarticular group, however, the incidence of death in that population in the 20 years prior to the last decade seems to have been around $14 \% .8,165$ The most common causes of death have been amyloidosis, infection, intractable heart failure, and accidents. ${ }^{124,164,165}$ Some accidents are related to the presence of arthritis.

Death rates from all causes are diminishing in all pediatric rheumatology clinics. ${ }^{124}$ In our own hospital, during the decade 1961-1970, there were five deaths. In the next decade, despite a greatly increased number of patients, there were only two deaths, one septic (gold neutropenia) and one arthritisrelated accident. Both deaths were theoretically avoidable. During the last decade there were no deaths, but death has on occasion been narrowly averted; these threatened patients have had disseminated intravascular coagulation (DIC), probably the most common cause of death now in arthritic children.

\section{Prevention of Death}

Recognition of DIC in JRA. It has been said that all children with systemic JRA have "low-grade" DIC; 166,167 if so, it is certainly not reflected in low fibrinogen levels or low platelet counts. On the contrary, systemic disease almost always is characterized by very high platelet counts and fibrinogen levels. If as a child becomes more ill the platelet count and fibrinogen fall to normal levels that child is endangered. ${ }^{168}$ Although we first recognized precipitation of this life-threatening syndrome by the second injection of gold, ${ }^{169}$ it has become apparent that it may follow introduction of any medication and on occasion be a complication of viral illness. ${ }^{170-172}$ Although not recognized as DIC, cases were first reported by Kornreich in 1971,173 and in that report one may find the features which we now recognize as characteristic: (1) a new rash-not typical of JRA, (2) lymphadenopathy and hepatosplenomegaly, (3) elevation of SGOT/PT and, in some cases, bilirubin, (4) thrombopenia, (5) lowering of ESR, and (6) paucity of arthritis. Other features seen in some patients include diarrhea; adult respiratory distress syndrome (ARDS); ${ }^{174}$ rhabdomyolysis with elevation of CPK, myoglobinuria, and renal manifestations; drowsiness; and coma. ${ }^{175}$ Even in highly specialized centers $7 / 11$ of these patients have died. Our experience and that 
of others suggest that prompt recognition and vigorous management of this syndrome results in (almost) 100\% survival. ${ }^{176}$ Management includes (1) stopping all medications, (2) high-dose intravenous steroid, 170,177,178 (3) providing vitamin $\mathrm{K}$ and coagulation factors (fresh-frozen plasma), and (4) intensive care. Some patients recover from this syndrome spontaneously. However, if death is to be avoided altogether, probably all patients with this syndrome will have to be treated vigorously. Once intractible GI bleeding or intracranial bleeding has occurred, treatment is likely to be ineffective.

We have seen one patient with dysfibrinogenemia without thrombopenia which resulted from aspirin treatment of a child with JRA and an unrecognized choledochal cyst. ${ }^{179}$ Erythrophagocytic syndromes ${ }^{100-102}$ and angioimmunoblastic lymphadenopathy with dysproteinemia ${ }^{180,181}$ are similar to this coagulopathy.

Prevention of Addisonian Crisis. Patients taking corticosteroids required steroid support for surgery, in case of accident, or for the stress of serious infection. Missed doses due to vomiting in stress situations and forgetting to provide parenteral steroids in stress continue to be an avoidable cause of death.

\section{Treatment of Still's Disease}

Treatment of Fever and Arthritis. Salicylates were obtained from plants and used as antipyretics and for analgesia by Hippocrates over 2000 years ago. ${ }^{182}$ Easily dissolved palatable $81-\mathrm{mg}$ tablets are available and very convenient for parents of infants and young children. Ordinary 325-mg generic aspirin tablets provide the cheapest nonsteroidal anti-inflammatory therapy (Table 3.7). Buffering often prevents the achievement of constant therapeutic salicylate levels (Fig. 3.12) ${ }^{183}$ and has not been shown to prevent gastric erosion. ${ }^{184}$

The mechanisms of action of aspirin remain a great mystery. In 1971, Vane reported that aspirin inhibits the synthesis of prostaglandins, hormonelike substances that can cause redness, swelling, and fever. ${ }^{185}$ Other anti-inflammatory actions of aspirin are probably directed at lymphokine action on target cells or lymphokine production by lymphocytes. ${ }^{186} \mathrm{It}$ is unlikely that any one action accounts for the total response.

The anti-inflammatory effects of aspirin in arthritis are only demonstrable at high dosage. ${ }^{187}$ In adults, $4 \mathrm{~g}$ daily is required; similar studies have not been performed in children. Analgesia is achieved by considerably lower doses.

Aspirin remains a commonly used treatment in Still's disease and is usually only effective in controlling fever and arthritis after a salicylate level of between 25 and $30 \mathrm{mg} / \mathrm{dl}$ is achieved. A few patients require and tolerate slightly higher levels (Figs. 3.13 and 3.14). ${ }^{188,189}$ In systemically ill patients, 
Table 3.7. Relative Cost of Antirheumatic Drugs

\begin{tabular}{lcccc}
\hline Drug & Size $(\mathrm{mg})$ & $\begin{array}{c}\text { Cost per } 100 \\
(\$)\end{array}$ & $\begin{array}{c}\text { Daily Dose } \\
(40 \mathrm{~kg})\end{array}$ & $\begin{array}{c}\text { Daily Cost to } \\
\text { Patient }\end{array}$ \\
\hline $\begin{array}{l}\text { Salicylates } \\
\text { Aspirin }\end{array}$ & & & & \\
Bayer timed- & 325 & 2.10 & 9 & .19 \\
$\quad$ release aspirin & 650 & 10.00 & & \\
Ecotrin & 325 & 7.90 & 8 & .40 \\
Bufferin & 325 & 9.70 & $8+$ & .63 \\
Baby aspirin & 81 & 7.00 & 35 & $.78^{*}$ \\
Trilisate & $500 / \mathrm{tsp}$ & $31.06(240 \mathrm{cc})$ & $5 \mathrm{tsp}$ & 2.45 \\
NSAIDs & & & & 2.59 \\
Naprosyn susp. & $125 \mathrm{mg} / \mathrm{tsp}$ & $52.27(480 \mathrm{cc})$ & $4 \mathrm{tsp}$ & \\
Indocin & 25 & 50.35 & 3 & 2.17 \\
Naprosyn & 250 & 74.55 & 2 & 1.51 \\
Clinoril & 150 & 91.25 & 2 & 1.49 \\
Nalfon & 300 & 56.85 & 5 & 1.83 \\
Motrin & 400 & 11.35 & 4 & 2.84 \\
Meclomen & 50 & 64.30 & 4 & .45 \\
Tolectin & 200 & 51.95 & 6 & 2.57 \\
Orudis & 50 & 92.15 & 3 & 3.11 \\
Ansaid & 50 & 71.80 & 3 & 1.86 \\
Feldene & 10 & 129.70 & 1 & 2.15 \\
Voltaren & 50 & 88.65 & 3 & 1.30 \\
Dolobid & 250 & 52.75 & 3 & 2.57 \\
\hline
\end{tabular}

* Rapid excretion may result in the need for increased dosage.

Table 3.8. Doses of Drugs and Frequency of Administration Required to Control Fever in Systemic JRA

\begin{tabular}{|c|c|c|c|}
\hline Regimen & Alone & $\begin{array}{l}\text { With Prednisone } \\
(0.6-1.0 \mathrm{~kg} \text { q.o.d.) }\end{array}$ & $\begin{array}{l}\text { Usual } \\
\text { Recommended } \\
\text { Dose }\end{array}$ \\
\hline Aspirin* & $\begin{array}{l}130-150 \mathrm{mg} / \mathrm{kg} / \mathrm{day} \\
(6 \text { doses })\end{array}$ & $\begin{array}{l}110-180 \mathrm{mg} / \mathrm{kg} / \mathrm{day} \\
\text { (Higher dose on "off- } \\
\text { prednisone" day)** }\end{array}$ & $90-110 \mathrm{mg} / \mathrm{kg}$ \\
\hline Tolmetin & $\begin{array}{l}30 \mathrm{mg} \mathrm{kg} \text { day } \\
(5 \text { doses })\end{array}$ & $40 \mathrm{mg} / \mathrm{kg}$ & $20-30 \mathrm{mg} / \mathrm{kg}$ \\
\hline Naproxen & $\begin{array}{l}20 \mathrm{~kg} / \mathrm{day} \\
(3 \text { doses })\end{array}$ & $25 \mathrm{mg} / \mathrm{kg}$ & $10-15 \mathrm{mg} / \mathrm{kg}$ \\
\hline
\end{tabular}

*Success depended on patient's ability to tolerate $130-150 \mathrm{mg} / \mathrm{kg} / \mathrm{day}$ with salicylate levels 25-39 in 6 doses (day and night).

** Subsequent patient has required the higher dose on the "on-prednisone" day.

(Reprinted with permission from Jacobs, ref. 198.) 


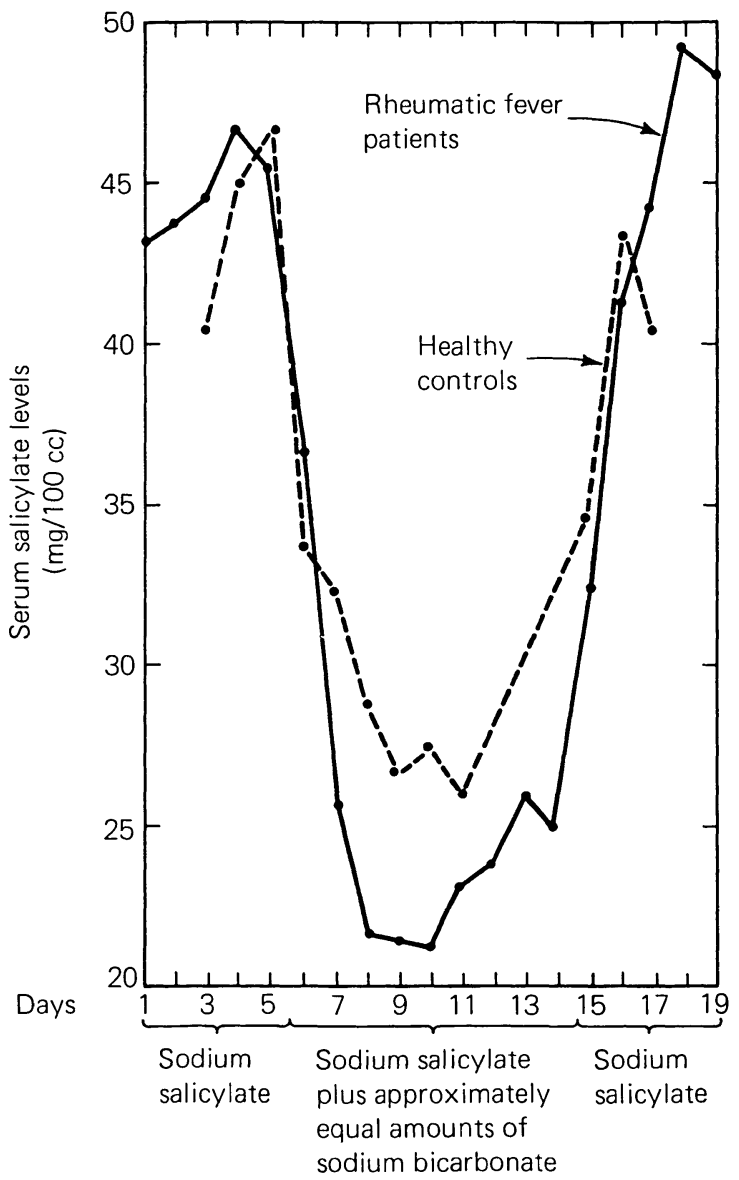

Figure 3.12. Dramatic decrease in salicylate levels caused by buffering with equal amounts of sodium bicarbonate. (Based on data of Smull et al., JAMA 125:1173-1174, 1944. From Gross M, Greenberg LA: The Salicylates: A Critical Bibliographic Review. Hillhouse Press, New Haven, 1948.)

these levels are rarely if ever obtained with less than $110 \mathrm{mg} / \mathrm{kg} / \mathrm{day}$, which is the customary starting dose (up to $3.6 \mathrm{~g}$ daily). Often a higher per-kilogram dose is required in young children. Some systemically ill children require and tolerate much higher doses to achieve a therapeutic level (Tables 3.8, 3.9); this is especially true in children receiving concomitant prednisone therapy (Fig. 3.15). One of our sickest patients with malabsorption and concomitant steroid therapy required $300 \mathrm{mg} / \mathrm{kg} /$ day; some children require six doses daily for control of fever.

In patients who tolerate an average dose without adverse symptoms and whose disease is controlled with that dose, salicylate levels need not be measured. However, in patients requiring dosage regulation to achieve control of the disease or in those who develop signs of salicylism, blood levels are measured $^{190,191}$ and the dose is adjusted. Salicylate levels reach their peak 9 days after the initiation of steady-dose therapy and tend to fall off slightly 
A

$$
\text { A }
$$

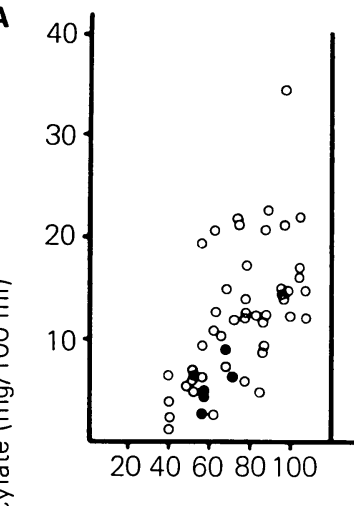

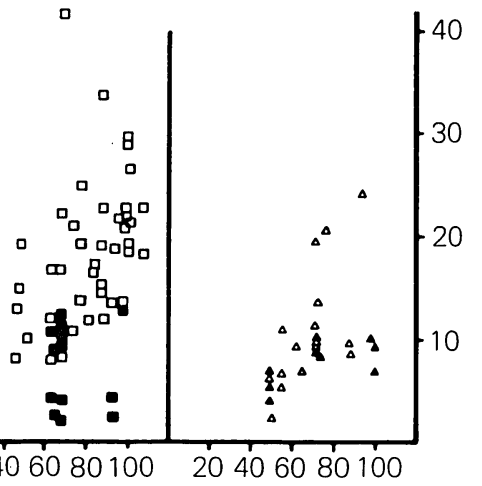

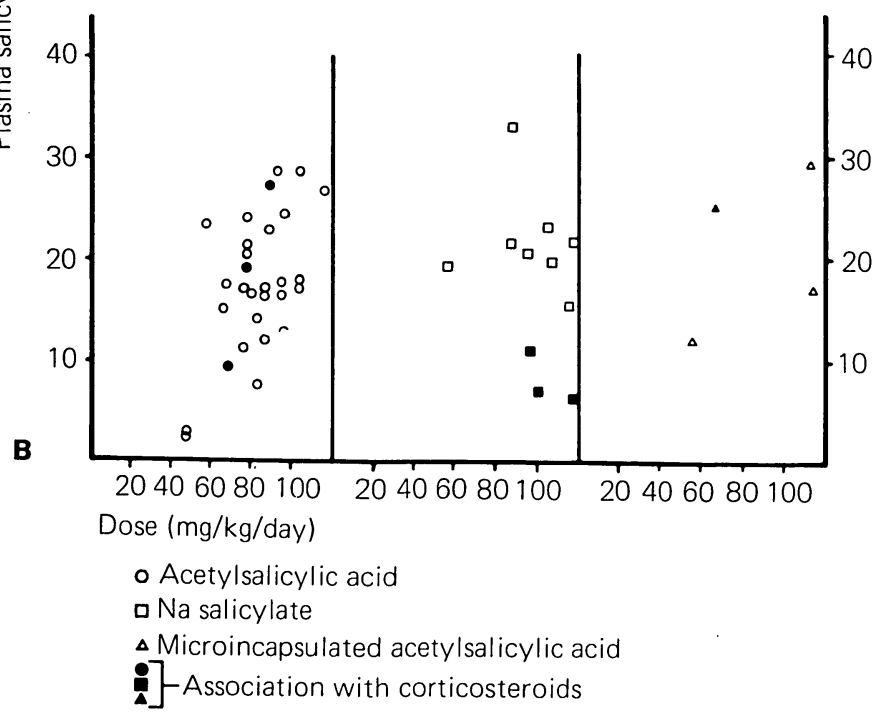

Figure 3.13. Great interindividual variability of plasma salicylate levels is shown in children receiving the same dose, both as outpatients $(\mathbf{A})$ and inpatients $(\mathbf{B})$. For example, in 14 patients taking sodium salicylate as outpatients, a daily dose between 99 and $106 \mathrm{mg} / \mathrm{kg}$ (mean, $101 \mathrm{mg}$ ) yielded plasma salicylate concentrations ranging from 15 to $30 \mathrm{mg} / \mathrm{dl}$ in different individuals; similarly, a daily dose of acetylsalicylic acid (ASA) between 78 and 83 $\mathrm{mg} / \mathrm{kg}$ (mean, $80 \mathrm{mg}$ ) in outpatients gave plasma salicylate levels varying from 6 to $24 \mathrm{mg} / \mathrm{dl}$ in different children. Note lowering of levels caused by additions of corticosteroids. (Reprinted with permission from Bardare et al. ${ }^{191}$ )

thereafter. ${ }^{192}$ At high serum levels, small-dosage increments may result in considerable increases in serum levels. ${ }^{193}$ We generally increase by no more than $10 \%$ at one time. Similarly, if toxicity occurs, a reduction in dosage of $10 \%$ is often adequate to reach a tolerable therapeutic level (Table 3.9). Levels vary widely in the same patients, ${ }^{191,193}$ often related to activity. More activity seems to reduce the serum level in a given individual. ${ }^{194}$ Noncom 


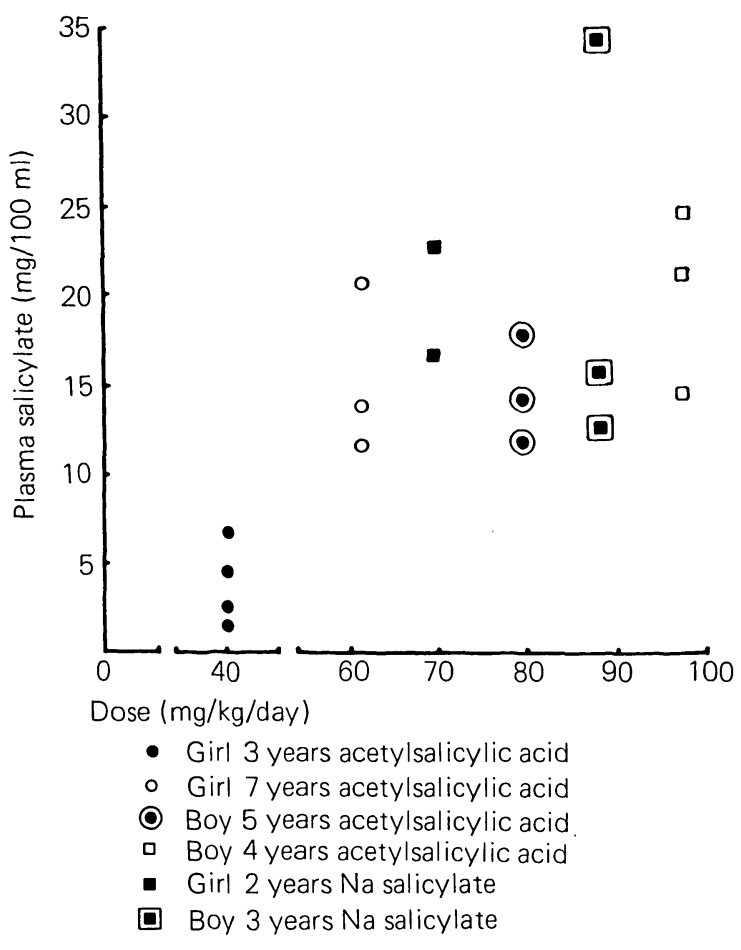

Figure 3.14. Daily plasma salicylate levels differ greatly in the same individual receiving the same dose as shown; in a 3-year-old boy, the level varied from 12 to $34 \mathrm{mg} / \mathrm{dl}$ despite steady dosing with 90 $\mathrm{mg} / \mathrm{kg}$ and obtaining specimens under identical circumstances regarding time after last dose. (From Bardare et al., ${ }^{191}$ Reproduced with permission of the authors and the editor of Archives of Disease in Childhood.)
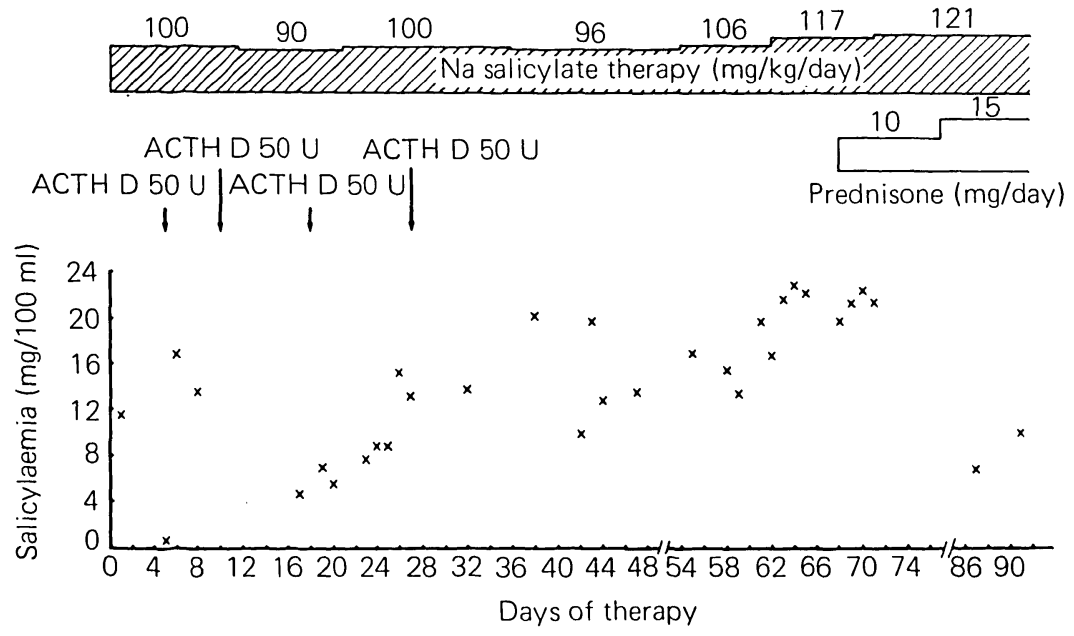

Figure 3.15. The addition of corticosteroids causes a prompt reduction in plasma salicylate levels. In a patient receiving daily prednisone, a high dose of salicylate $(121 \mathrm{mg} / \mathrm{kg} / \mathrm{day})$ yields only a very low salicylate level $(9 \mathrm{mg} / \mathrm{dl})$. It is impossible to prescribe appropriate amounts of salicylate to patients receiving concomitant corticosteroid therapy without measuring the salicylate level until the patient is receiving a steady dose of both drugs. (Reprinted with permission from Bardare et al. ${ }^{191}$ ) 
Table 3.9. Regimens Used to Control Fever in Systemic JRA (10 new patients)

\begin{tabular}{|c|c|c|}
\hline Regimen & $\begin{array}{l}\text { Number of children } \\
\text { controlled with this regimen }\end{array}$ & $\begin{array}{l}\text { Regimens which failed } \\
\text { in these patients }\end{array}$ \\
\hline Aspirin & $\begin{array}{l}2 \text { (sal level } 25-39 ; 130-150 \\
\quad \text { kg; } 6 \text { doses) }\end{array}$ & \\
\hline Tolmetin. & $1(30 \mathrm{~kg} ; 5$ doses $)$ & ASA, Tol $20 / \mathrm{kg}$ \\
\hline Naproxen & 2 (9-20kg; 3 doses) & ASA, Tol 20-40/kg \\
\hline Tolmetin + & $3(40 \mathrm{k} \sigma \cdot 06-1 / \mathrm{kr} a 0 \mathrm{~d})$ & ASA Nan $23 / \mathrm{kg}^{\circ} \cdot \mathrm{Tol} 30 / \mathrm{ko}$ \\
\hline Naproxen + & $J$ (4U kg, 0.0-1/kg q.o.d.) & ASA, Tol $20 \mathrm{~kg}+$ \\
\hline Prednisone q.o.d. & 1 (25kg; $1 / \mathrm{kg}$ q.o.d.) & Pred 2/kg q.o.d. \\
\hline $\begin{array}{l}\text { Aspirin }+ \\
\quad \text { Prednisone q.o.d. }\end{array}$ & $\begin{array}{l}1 \text { ( } 110-180 \text { alt kg; } 0.8 \mathrm{~kg} \\
\text { q.o.d.) }\end{array}$ & ASA, Tol $32 \mathrm{~kg}$, Nap 20/kg \\
\hline
\end{tabular}

From Jacobs, ref. 198, with permission.

pliance is also a common cause of variable levels. ${ }^{86}$ Tinnitus is not a reliable guide to salicylate levels. ${ }^{195}$ Careful regulation of salicylate levels protects the child from unnecessary exposure to more toxic agents. The important pharmacokinetic considerations for achieving optimal salicylate dosage are listed in Table 3.10 .

Nonsteroidal Anti-Inflammatory Drugs (NSAIDs). These medications are discussed in detail later in this chapter. By 1984 we had found that only $20 \%$ of new registrants with systemic JRA could be controlled with aspirin alone and only $30 \%$ more with naproxen $(19-25 \mathrm{mg} / \mathrm{kg} /$ day in eight hourly doses) ${ }^{196,197}$ or tolmetin (30 mg/kg/day in five doses) alone (Tables 3.8, 3.9). The other $50 \%$ of patients required prednisone $0.6-1.0 \mathrm{mg} / \mathrm{kg} /$ day every second morning before 7:30 A.M. plus aspirin or naproxen or tolmetin. The aspirin dose accompanying the steroid averaged $150 \mathrm{mg} / \mathrm{kg} /$ day and six doses were often required on the "off"-prednisone day; the tolmetin dose was 40 $\mathrm{mg} / \mathrm{kg} /$ day and the naproxen dose accompanying the steroid $25 \mathrm{mg} / \mathrm{kg} /$ day. Sometimes all of the NSAID is given during the period beginning 10 P.M. on the night of steroid administration or 6 A.M. the following morning and 2 A.M. the "on" day; for some patients this means the actual dose given during the $24 \mathrm{~h}$ of NSAID alternating with a single prednisone dose is triple the usual recommended daily dose. These techniques, developed over the past 7 years, have been extremely helpful to us in achieving control of patients without the need for daily steroids.

Slower-acting Agents. If despite maximally tolerated salicylate or NSAID treatment, a patient cannot attend school or lead a relatively normal life, corticosteroids are necessary (Fig. 3.16). Standard "slower-acting" agents, 
Table 3.10. Important Pharmacokinetic Considerations for Achieving Optimal Salicylate Dosage

Pharmacokinetic studies show half-life varies with tissue saturation.

High-dose steady state not achieved for 7-9 days.

At high levels, small dosage changes cause large variations in serum levels.

Urinary excretion varies greatly with small changes in urine $\mathrm{pH}$.

Scrum levels are greatly increased by small decreases in urine $\mathrm{pH}$.

Serum levels are greatly decreased by small increases in urine $\mathrm{pH}$.

Great interindividual variation in dose is required to achieve the same level.

Great intraindividual variation in serum levels occurs at different times in the same patient on the same dose.

Great variation in tolerated and effective levels in different children.

Addition of corticosteroids to therapeutic regimen causes reduction in the salicylate level and requires considerable increase in salicylate dose to achieve prior level.

Removal of corticosteroids from the therapeutic regimen causes increase in the salicylate level and poisoning if not carefully monitored.

Systemically ill and anemic patients often require high doses due to malabsorption and/or altered metabolism of salicylate.

Note: Salicylate increases blood alcohol concentrations. ${ }^{200}$

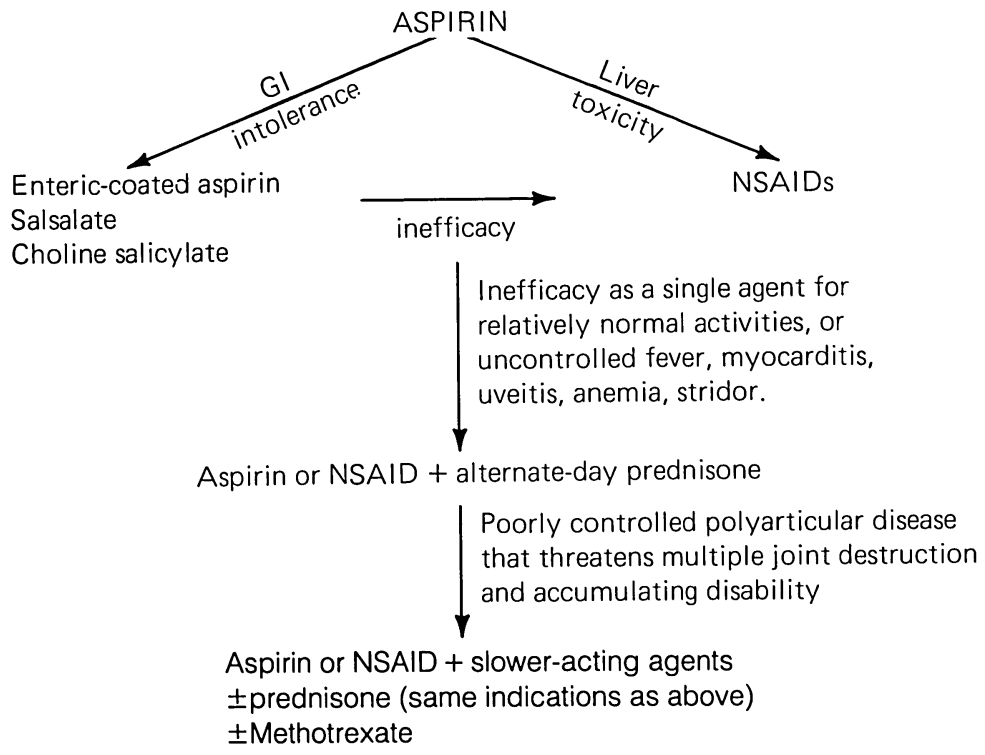

Figure 3.16. Treatment module for childhood arthritis. 
Table 3.11. Indications for Systemic Corticosteroid Therapy in JRA

Fever that cannot be controlled with any NSAID

Myocarditis

Tamponade not controlled with intrapericardial steroid injection

Uveitis not controlled with local steroids

Intolerable anemia

Inability to ambulate

Disseminated intravasular coagulation (DIC)

Acute laryngeal stridor

discussed in the section on polyarticular disease, may also help in the control of arthritis in systemic-onset patients, ${ }^{199}$ but not generally during the acute phase.

Corticosteroids. When fever and polyarthritis cannot be controlled with maximum regimens of nonsteroidal anti-inflammatory agents, it may be necessary to add prednisone to the regimen (Tables 3.9 and 3.11). It is always preferable and usually possible to use an alternate-day regimen. Most children with disease that requires steroids can be adequately managed with full doses of aspirin or another NSAID and a "boost" of a small dose of prednisone every second morning (10-15 mg god). Very sick children who cannot be controlled with ordinary alternate-day steroid supplements usually will respond to surprisingly large alternate-day doses of prednisome (3 $\mathrm{mg} / \mathrm{kg} / \mathrm{dose}$ ) or whatever dose is required to control their unimaginable pain, fever, and anemia. The regimen must be individualized for each child. Monitoring of prednisone blood levels is complicated and not yet generally available. ${ }^{201}$

The adverse effects of long-term glucocorticoid therapy are well known to the clinician (Table 3.12). ${ }^{202}$ Alternate-day steroid therapy has provided the physician with the ability to slow progression of the disease and avoid bed-chair crippling with an acceptable amount of steroid toxicity. Even "astronomically" high alternate-day doses are better tolerated than daily steroids. ${ }^{203-206}$ Corticosteroids, therefore, if administered skillfully, are the tool that avoids bed-chair crippling, whereas if misused, these same drugs can cause increased crippling or even death.

Alterations in Salicylate Dosage Required after Introduction of Supplemental Corticosteroids. It has been repeatedly demonstrated that salicylate levels fall after the introduction of corticosteroids to the therapeutic regimen (Figs. 3.13, 3.15, and 3.17). Even a single injection of steroids into a joint lowers salicylate levels dramatically for up to 5 days. ${ }^{209}$ Therefore, when adding prednisone, it is necessary to redetermine the salicylate level and increase the dose of salicylates to maintain maximum-tolerated serum level. ${ }^{191,210}$ If the physician is not alert to this phenomenon, there may be a 
Table 3.12. Adverse Effects of Glucocorticoid Therapy

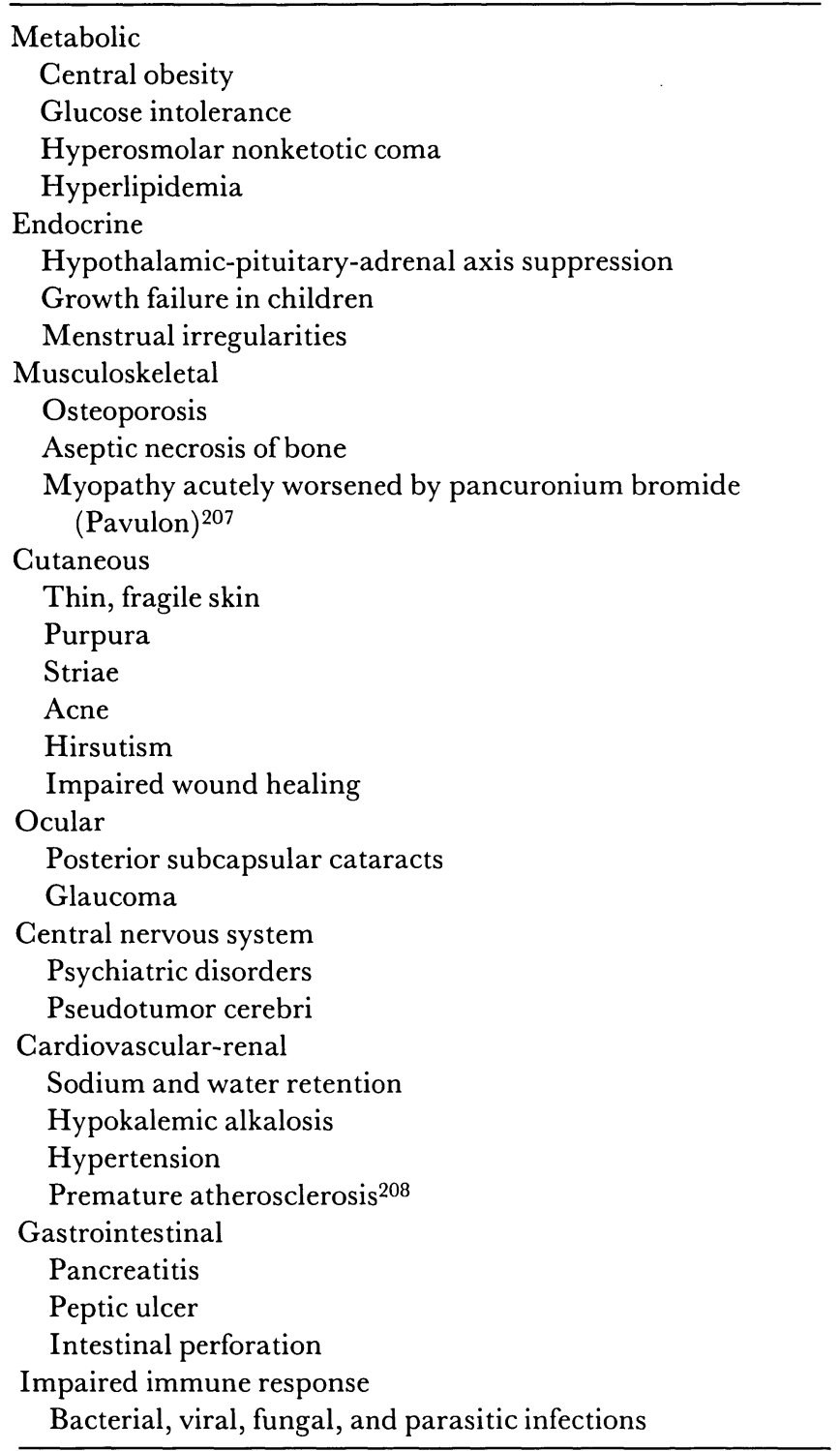

(Modified with permission from Nelson and Conn, ref. 202, p. 766.)

tendency to continuously increase the prednisone dosage, resulting in further lowering of the salicylate level and the need for more prednisone. Although this principle has been repeatedly emphasized, our experience suggests that it is repeatedly neglected even by experienced rheumatologists.

It is equally important to be alert to the potential for salicylate poisoning 


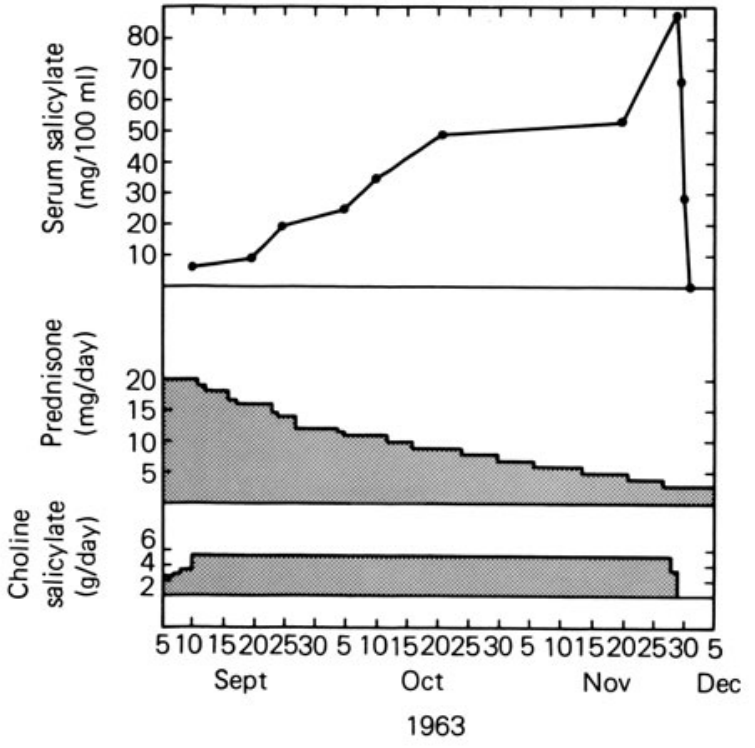

Figure 3.17. Dramatic progressive salicylate poisoning of a 5-year-old boy with JRA produced by gradual withdrawal of prednisone while maintaining a steady dose of salicylate. Note that initially, while receiving prednisone (1 $\mathrm{mg} / \mathrm{kg} /$ day), a salicylate level of only $10 \mathrm{mg} / \mathrm{dl}$ was achieved despite the administration of about $250 \mathrm{mg}$ of salicylate $/ \mathrm{kg} /$ day. (Reprinted with permission from Klinenberg and Miller, ref. 210. Copyright 1965, American Medical Association.)

when lowering the dose of prednisone administered to children also receiving aspirin. In this circumstance, salicylate levels rise, and the dose of aspirin may have to be adjusted downward to avoid poisoning (Fig. 3.17). ${ }^{191,210} \mathrm{We}$ teach these principles to the parents of our patients, who can then determine when to measure the levels and are often able to make the necessary adjustments in dosage without instructions from the physician.

Salicylate Toxicity. Flavored tablets must be dissolved (not chewed) and ingestion must be followed by liquid to avoid dental carries. ${ }^{211}$ Children tolerate aspirin well, and dyspepsia is rare. However, for many children with systemic and polyarticular disease, effective treatment demands a salicylate level close to toxicity. ${ }^{188-190}$

If adequate control of a child's arthritis requires maximum-tolerated doses of aspirin, it goes without saying that the parent must be aware of the signs of salicylate poisoning. Contrary to medical folklore, tinnitus does not correlate well with salicylate levels. ${ }^{195}$ When aspirin treatment is begun, we advise the parent of the three cardinal signs of too much aspirin (Table 3.13):

1. Change in personality for the worse. In general, after institution of aspirin therapy in the arthritic child, there is improvement in disposition with relief of pain and inflammation. After a week, if aspirin levels increase to toxic proportions, there will be a behavioral regression, with irritability and emotional lability, requiring measurement of the level, prompt report $(2 \mathrm{~h})$, and reduction in dosage.

2. Fast breathing. Although all children with high salicylate levels breathe more rapidly than normal, a greatly increased respiratory rate simulating 
Table 3.13. Cardinal Signs of Salicylate Toxicity

Change in personality for the worse (irritability, lability, depression, withdrawal)

Fast breathing ("croup")

Nausea (anorexia) or vomiting

dyspnea or croup should be assumed, in these children, to represent salicylate poisoning, requiring prompt measurement of the serum level prior to any further salicylate administration.

3. Nausea and vomiting. Most children with systemic JRA requiring maximum salicylate levels will have improved appetites as fever and pain are controlled. However, if the salicylate level then climbs to toxic proportions, the improvement in appetite will be replaced by anorexia followed by nausea and, if the dosage is not then reduced, by repetitive vomiting. Sometimes a child vomits only occasionally, usually shortly after taking the medicine, as the sole manifestation of too much aspirin, presumably because levels are too high only intermittently. Parents and physicians can be alert to this potential by recognizing that any vomiting requires measurement of the salicylate level. Parents often assume vomiting to be a result of a viral illness. No such assumption is permissible unless the salicylate level has been measured and demonstrated to be nontoxic. Viral illnesses are common in children and may result in lowering of salicylate levels due to vomiting of medications or increases in levels due to relative dehydration from the illness; we cannot distinguish one from the other without the salicylate level. Our office and hospital staff is trained to obtain these levels at any time at the request of any of our patients (without formality) and to promptly provide the results in our absence to the parents, who are aware of the desired therapeutic levels and the technique of dosage omission and regulation. In order to care for arthritic children adequately, salicylate levels must be available $24 \mathrm{~h}$ /day with 2-h reporting. Less than this will result in increased use of more toxic secondary agents in these children. A technique for microsalicylate determinations ensures greater patient/parent compliance in toddlers. ${ }^{190}$

Reye's Syndrome. Almost everyone interprets the available data as supporting an increased risk of Reye's syndrome in children taking aspirin and, perhaps, nonaspirin salicylates. ${ }^{212}$ In certain centers caring for arthritic children, Reye's syndrome occurs in frightening numbers of their patients; ${ }^{213,214}$ in other centers, such as our own, there have been no cases despite 30 years of high-dose aspirin administration to large numbers of patients. This remains an enigma.

Parents now stop aspirin when they know their children are exposed to varicella. Influenza may be asymptomatic in more than $50 \%$ of cases and children are frequently exposed to others with viral illnesses which could be 
influenza; stopping medication with each viral exposure is impractical, and there is no evidence at all that stopping the aspirin at the time of exposure reduces the risk. The need to administer influenza vaccine to arthritic children yearly, sometimes with considerable adverse reaction and always with inconvenience and expense of yet another needle, discourages most parents in New York from choosing aspirin as the drug of choice for their arthritic children though it may be the safest agent. ${ }^{215}$

Bleeding as Result of Salicylate Administration. This particular toxic side effect of aspirin administration deserves special consideration. Tiny doses of aspirin have long been recognized to have the potential to cause bleeding in those with hemophilia. ${ }^{216}$ Occasionally, patients with bleeding disorders secondary to abnormalities of platelet aggregation report bleeding with aspirin. ${ }^{217}$ What was not initially recognized was that this might be paradoxically dose-related. Recent evidence suggests that this effect may not be seen in patients receiving high doses of aspirin. ${ }^{218}$ Bleeding time may also not be further increased in such patients by the nonacetylated salicylate preparations, salicylsalicylic acid (salsalate; Disalcid), and choline magnesium salicylate (Trilisate; see below). ${ }^{219}$

Easy bruisability may result from prolongation of the prothrombin time in children receiving chronic aspirin therapy. ${ }^{220}$ Even in children in whom the determination of prothrombin time is normal, vitamin $\mathrm{K}, 5 \mathrm{mg}$ orally daily, may be reported by patients to reduce bruisability and nosebleeds.

Significant gastrointestinal hemorrhage is a rare complication of aspirin administration in children. Controversy exists over whether such hemorrhage occurs only in those with primary coagulation defects or whether it can occur in normals as well.221,222 When such hemorrhage occurs in children treated with aspirin alone, it is generally a result of gastric bleeding.

Salicylates and the Liver. When the SGOT/SGPT tests became available, it was observed that patients with rheumatic fever being treated with large doses of aspirin frequently had elevated levels of these "liver" enzymes. No harmful effects were reported associated with these chance laboratory observations. ${ }^{223}$ Subsequent studies in arthritic children, coincident with the institution of machine determinations of multiple blood chemistries, revealed that unrecognized asymptomatic elevations of "liver" enzyme (below 400 units) occurred with aspirin therapy in $25 \%$ of children with arthritis, were often dose-related, tended to disappear with reduction of dose or with time alone, and generally seemed to have no pathologic consequences. ${ }^{224}$ Nevertheless, it remains possible that subtle symptoms or liver damage might occur in such children. ${ }^{225}$ It seems reasonable not to discontinue aspirin and institute more toxic agents solely because of chance laboratory observations, but if equal control of the arthritis can be achieved with agents of equal or less toxicity without these abnormalities, it would seem the prudent course to change to those agents. Thus, only in the case of severe life-threatening eleva- 
tions in enzymes would it be worth instituting daily corticosteroids as an alternative to aspirin, but at even lower levels of enzyme abnormality, consideration should be given to trying other nonsteroidal anti-inflammatory agents that do not raise the level of SGOT/SGPT. These drugs may be instituted on the same day the aspirin is withdrawn, and the liver enzyme abnormalities, if caused by aspirin, generally are eliminated within a few days. ${ }^{226}$

A small number of children develop symptomatic hepatotoxic effects of salicylate with elevations of SGOT/SGPT over 1000 units, tender hepatomegaly, vomiting, and a prolonged prothrombin time. Continuation of aspirin therapy may result in serious, even fatal, hepatotoxicity. In most studies, this intolerance occurs more frequently in systemically ill patients, especially those with little or no arthritis, who may represent a different subset with more primary hepatic involvement.

It is our policy to measure SGOT/SGPT in all patients prior to instituting therapy and during the period of dosage regulation. These enzymes are also measured in patients with abdominal pain or malaise and in those manifesting symptoms of salicylate toxicity.

Occasionally, using choline salicylate in lieu of other salicylate preparation results in less hepatotoxicity. ${ }^{189}$ In some children who cannot tolerate salicylates, other nonsteroidal anti-inflammatory agents can be substituted successfully without hepatoxicity. However, while these agents are rarely hepatotoxic, the same patients who cannot tolerate salicylates sometimes cannot tolerate these agents, either. In other children, adequate control of fever is not achieved with these agents, at least with currently accepted dosage and with our inability to measure serum levels to identify those who might require a higher dose to achieve a meaningful serum level (due to altered absorption, metabolism, or excretion). A stratagem that we generally find effective in very difficult situations in which serious salicylate hepatotoxicity occurs and no other agent provides adequate control is the use of alternateday steroids plus aspirin. Some children will tolerate a required and otherwise intolerable salicylate level when taking relatively small doses $(1 \mathrm{mg} / \mathrm{kg}$ ) of alternate-day prednisone in addition to salicylate. The mechanism by which the prednisone protects the liver in this situation is speculative.

Gastritis and Ulcers. Within a few minutes of aspirin ingestion, gastric acidity increases, presumably as a result of back-diffusion of hydrogen ions in the mucosa. Whether salicylate administered systemically has a similar effect is controversial. ${ }^{227}$ Animal experiments suggest that the mucosa develops some resistance to this effect with chronic administration. ${ }^{222}$

The weight of evidence suggests that gastric and duodenal ulcers are associated with heavy aspirin usage. ${ }^{222,227 a}$ There is some evidence of increased susceptibility to ulcers in patients with RA even without salicylate treatment. In our experience, duodenal ulcers are not uncommon in arthritic children. While the role of aspirin in the causation of these ulcers may be controversial, there is no question that patients with peptic or duodenal ulcer 
disease are less tolerant of aspirin than normal and suffer from worse dyspepsia than others. ${ }^{222}$

We have seen no instance of major gastrointestinal bleeding from duodenal ulcer in an arthritic child treated with aspirin alone; the same cannot be said for combinations of aspirin with corticosteroids and indomethacin, which have in one instance each resulted in uncontrollable hemorrhage requiring gastrectomy in two children under the age of 5. These experiences have sensitized us so that we treat ulcer symptoms by changing to entericcoated aspirin and providing frequent milk feeds, sucralfate, and antacids if there is nocturnal ulcer pain. In the presence of documented ulcers $\mathrm{H} 2$ blockers are also administered. Misoprostol, a protaglandin E1 analog prescribed for the prophylaxis and healing of peptic ulceration associated with NSAID use, has not been studied in children but may be useful. ${ }^{228}$

The toxic effects of salicylates have been overemphasized when compared to their therapeutic benefits and the billions of tablets consumed each year. ${ }^{222}$ Aspirin has been accused of causing analgesic nephropathy, but there is no proof of a single case in a child receiving aspirin alone. ${ }^{130,131,229}$ Although hearing may be temporarily reduced, especially at high frequencies, there is no evidence that permanent hearing loss results from chronic aspirin administration. There are a great many other metabolic, endocrinologic, hematologic, and immunologic effects of aspirin administration that are adequately reviewed elsewhere. ${ }^{182,230}$

Special Aspirin Preparations. There is increasing evidence that entericcoated aspirin produces fewer gastroduodenal erosions. ${ }^{231}$ Some children with dyspepsia who cannot tolerate regular aspirin will tolerate entericcoated aspirin and absorb it well, attaining adequate and reliably constant salicylate levels. ${ }^{232}$ In these youngsters, there is no reason not to use entericcoated tablets. ${ }^{233}$ Not all enteric-coated tablets are equally well absorbed. 234,235 In one study, Ecotrin was best absorbed, and this and Easprin (975 mg) are the sole enteric-coated tablets in use in our clinic. We have sometimes seen even these tablets undigested and whole in the stool of our patients.

Sustained-Release Aspirin. These tablets are constructed so as to provide sustained release over an 8-h period, presumably providing better nighttime and early morning blood levels and fewer peaks and troughs. This is achieved by partial absorption in the stomach and the rest in the small intestine. ${ }^{236}$ Controversy exists as to whether sustained serum salicylate levels are really better achieved during chronic administration of these costly tablets. However, some patients with dyspepsia who do not absorb entericcoated tablets will do well with sustained-release tablets.

Older children hate to take medicines in school both because the other children will "see" and because many schools require the children to go to the nurse's office to have their medicine administered to them. These anti- 
quated countertherapeutic school regulations infantilize and isolate our handicapped children, who we wish to become responsible for their own care and self-sufficient despite their handicaps. American Academy of Pediatrics policy for school health advocates children being permitted to take their own medications in school. ${ }^{237}$

Any medication with a long-enough half-life or a sustained-release tablet which allows teenagers to avoid taking medicines in school may be advantageous and worth considerable additional expense. In adults, better compliance has been shown to be achieved with less frequent dosage schedules, resulting in better control of arthritis by drugs with a long half-life when compared with equipotent medications that require more frequent administration. ${ }^{238}$

Other Forms of Salicylate. Salsalate and choline magnesium trisalicylate are hydrolyzed in vivo to salicylic acid and so presumably have comparable activity to aspirin. They are nonacetylated and therefore presumably do not affect platelet aggregation. ${ }^{219}$ Salicylate levels can be determined and regulated just as when aspirin is administered, providing a mechanism to determine compliance and optimal dosage. These preparations are much more expensive than aspirin (Table 3.7) but have provided control of disease in some children who could not take aspirin.

Salsalate (salicylsalicylic acid), marketed in the United States under the trade name Disalcid, is available only in nondissolvable tablet form, with each tablet equal to $500 \mathrm{mg}$ of salicylate. Dosage regulation is the same as for aspirin; presumably, medication can be administered at 8-h intervals. Salsalate commonly produces abnormalities in routine thyroid function tests similar to those found in central hypothyroidism. ${ }^{238 a}$

Choline salicylate is also available in tablet form as choline magnesium trisalicylate. This product (Trilisate) is reported to be useful in some patients who cannot tolerate aspirin but has not been tested in children. Tablets ( 500 $\mathrm{mg}, 750 \mathrm{mg}$, and $1000 \mathrm{mg}$ of active ingredient) contain more than half of the salicylate as the magnesium salt, which might cause diarrhea in some children. A palatable syrup (500 $\mathrm{mg} / \mathrm{tsp}$ ) is available.

While choline salicylate liquid has been very useful in pediatric rheumatologic practice, its presence in the household is akin to the threat of oil of wintergreen, another potent salicylate liquid that has resulted in death as a result of toddler ingestion. We tend not to prescribe choline salicylate when there are toddlers in the home who may accidentally ingest it and are careful to warn parents to keep it locked up at all times.

\section{Treatment of Pericarditis and Myocarditis}

Mild pericarditis can often be successfully treated with aspirin alone. ${ }^{86,88,90,91,93}$ Colchicine may also be helpful. ${ }^{239}$ However, when pericar- 


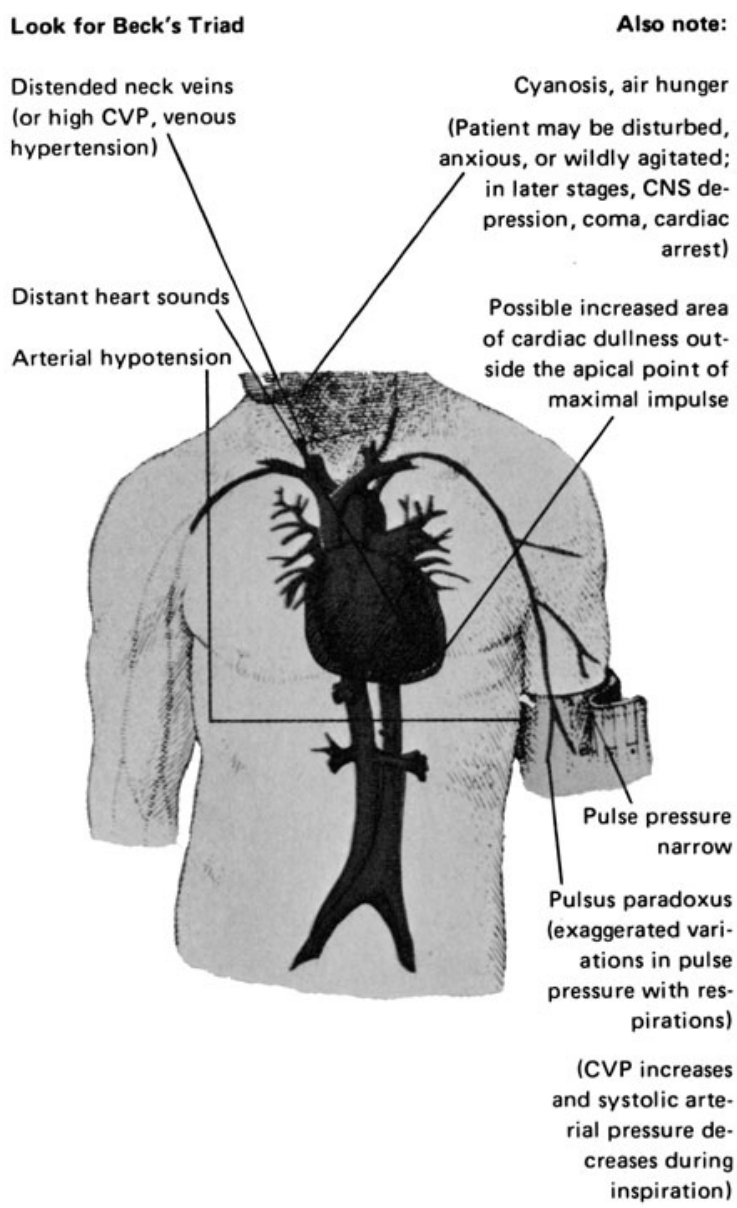

Figure 3.18. Pericardial tamponade may occur in children with systemic JRA and demands prompt diagnosis and therapy. Of 80 patients with tamponade reported by Shoemaker, $87 \%$ had one or more signs of Beck's triad, but only $35 \%$ showed all three. These findings may occur rather late in the course. If treatment is delayed until all the classic signs of the triad are present, cardiac arrest is likely to intervene. (Reprinted with permission from Hospital Medicine, November 1978, copyright by Hospital Publications, Inc.)

ditis and myocarditis are life-threatening (Fig. 3.18), prednisone should be instituted promptly ( $2 \mathrm{mg} / \mathrm{kg} /$ day in four divided doses $) .86,88,90-92,94$

When steroids are to be administered to patients in congestive heart failure, it is useful to give an injection of furosemide $1 \mathrm{~h}$ prior to the first dose of steroid. This helps avoid further fluid overload. Potassium losses may be increased by both diuretics and steroids. Supplemental potassium may be given if necessary. Miller has suggested that death may result from digitalisinduced arrhythmia in these children. ${ }^{91}$ Patients with myocarditis are very prone to arrhythmia, and digitalis should, therefore, be avoided. ${ }^{240}$

Aspirin is not given initially to these patients because of the risk of increasing cardiac failure due to the increased metabolic rate induced by aspirin. After congestive heart failure has been controlled with prednisone, aspirin therapy is instituted. During concomitant steroid therapy, large doses of aspirin may be required to achieve an adequate salicylate level. The change 
to alternate-day prednisone (usually $150 \mathrm{mg}$ qod) is then made. The salicylate dosage may have to be gradually reduced if, due to reduction in steroid dosage (see above), serum salicylate begins to rise to toxic levels. This regimen allows rather prompt discharge from the hospital with gradual reduction of alternate-day prednisone (maintaining steady serum salicylate levels) over a period of several months, avoiding rebound exacerbations.

We have experience so far with only one case treated with intrapericardial injection of corticosteroids as the sole treatment for pericardial tamponade in an arthritic child but have also used this regimen as an emergency supplement. This technique was reported initially by Scharf et al. in $1976^{241}$ and is based on considerable reported experience in adults with tamponade associated with renal dialysis. ${ }^{242,243}$ In our case, we injected $200 \mathrm{mg}$ of triamcinolone hexacetonide (Aristospan $20 \mathrm{mg} / \mathrm{cc}$ ) at the time of tamponade, continuing high-dose oral aspirin but giving no systemic steroids. Removal of fluid at the time of the pericardiocentesis relieved the immediate problem; following the injection of steroids, there was no reaccumulation of fluid (Fig. 3.19). Had there been reaccumulation, we were prepared to offer a second injection. While this treatment is unusual, it does seem to offer a simple, safe, and effective bedside procedure in place of months of oral prednisone administration. We have used this approach in Kawasaki disease and the postpericardectomy syndrome and are determined to use it as our first approach to all children with tamponade from JRA provide the fluid is anterior so the procedure can be safely accomplished.

\section{Treatment of Laryngeal Stridor}

When systemic JRA presents with airway (cricoarytenoid) obstruction, corticosteroids should be promptly administered. After the obstruction has been relieved, salicylates are introduced and steroids gradually weaned away. ${ }^{114}$

\section{Polyarticular Juvenile Rheumatoid Arthritis}

All forms of arthritis may be polyarticular both in onset or course. The real significance of "polyarticular" is the implication of symmetrical destructive disease in many joints (Fig. 3.20). Later age of onset and involvement of many joints of the fingers, hands, and wrists are associated with poorer prognosis than polyarticular disease primarily limited to the lower extremities. ${ }^{244,245}$ These prognostic determinants are the same in JRA and in young adult arthritics. ${ }^{246}$

Adverse prognosis in polyarticular JRA is most closely correlated with the consistent presence of significant amounts of rheumatoid factor. ${ }^{57} \mathrm{It}$ is thus useful to subdivide polyarticular-onset JRA into "seropositive" and "seronegative" subsets. 


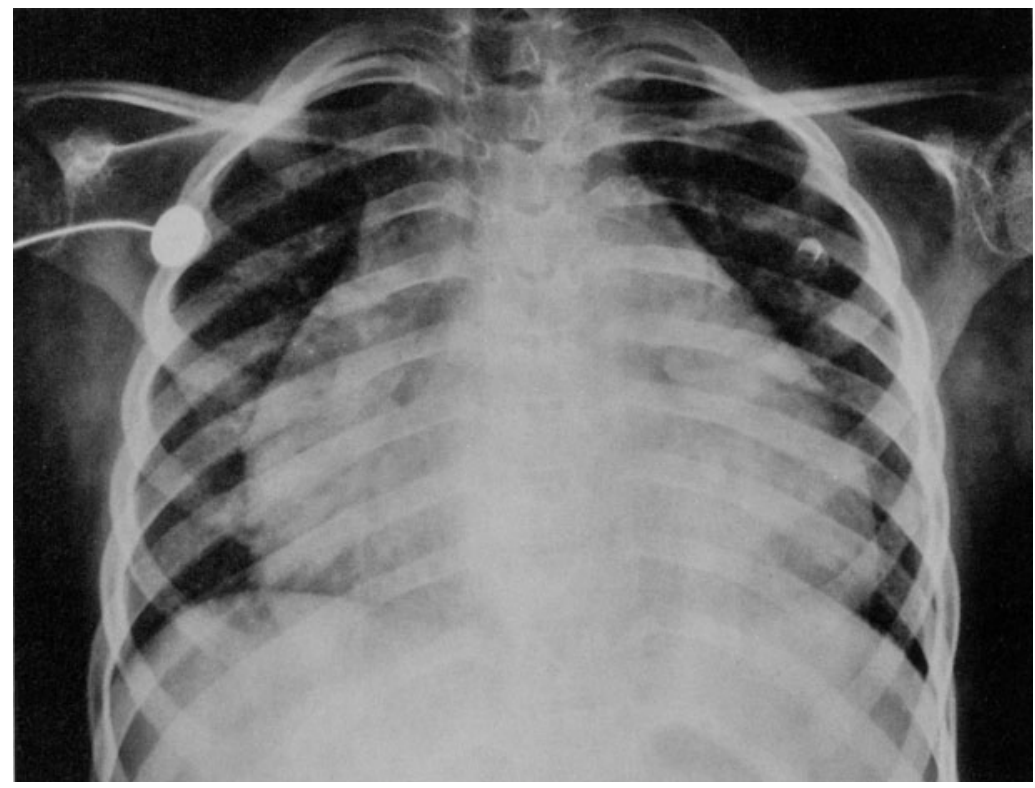

A

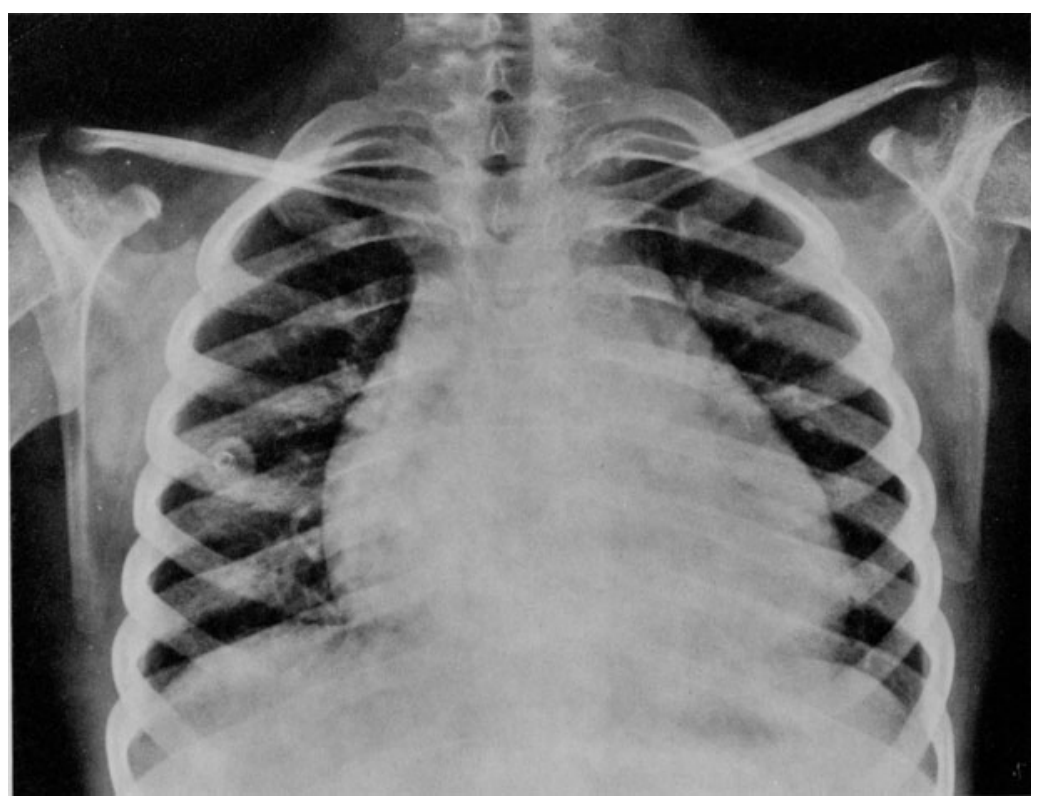

B

Figure 3.19. (A) Severe cardiomegaly shown in a chest film of a child with systemic JRA with pericarditis and tamponade despite aspirin therapy. (B) Repeat film 2 days later, after injection of depot steroids into the pericardial sac. No other treatment was necessary; the patient continued to receive aspirin, and all evidence of pericarditis gradually disappeared. 


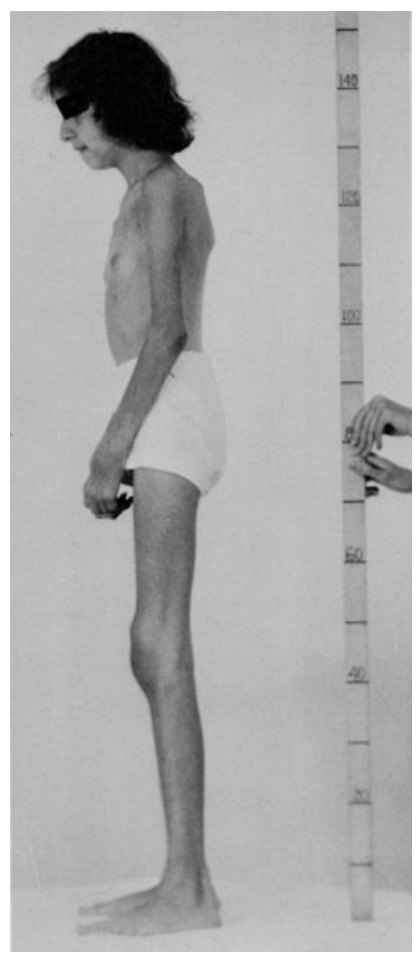

Figure 3.20. Poorly controlled polyarticular JRA affecting essentially all joints symmetrically and causing depression, delayed puberty, and dwarfing of this 13-year-old girl.

\section{Epidemiology and Prognosis}

Polyarticular Seropositive JRA. (Childhood Onset Classic Adult RA). ${ }^{16}$ Most arthritic children with consistently significantly positive tests for rheumatoid factor are girls, have the onset of disease after age 8 , have rheumatoid nodules, do not remit, ${ }^{248}$ and have severe progressive disease with extensive radiographic changes. In one series, after an average of less than 5 years, $50 \%$ had reached class III or IV disability ${ }^{5}$ (Table 3.14 ). The childhood early onset of this adult form of RA is thus associated with a very poor functional prognosis, indicating that girls affected this early have a special propensity for the disorder. This group represents less than $7 \%$ of the total JRA population in older reports and less than $3 \%$ of arthritic children currently being registered in our clinic. In both adults and children this subset of RA has been associated with HLA-DR4, ${ }^{249}$ and severity correlates with levels of both IgM and IgG rheumatoid factor. ${ }^{250}$ Aggressive therapy is necessary from the moment of onset. ${ }^{251}$

Polyarticular Seronegative JRA. Previously reported series of seronegative polyarticular JRA include some patients who have what we would now term 
Table 3.14. Classification of Functional Status in Rheumatoid Arthritis (ACR) ${ }^{\star 247}$

\begin{tabular}{ll}
\hline Class & Characteristics \\
\hline I & $\begin{array}{c}\text { Completely able to perform usual acitivities of daily living (self-care, } \\
\text { vocational, and avocational) } \\
\text { Able to perform usual self-care and vocational activities, but limited in } \\
\text { avocational activities }\end{array}$ \\
II & $\begin{array}{c}\text { Able to perform usual self-care activities, but limited in vocational and } \\
\text { avocational activities } \\
\text { III } \\
\text { Limited in ability to perform usual self-care, vocational, and avocational } \\
\text { IV } \quad \text { activities }\end{array}$ \\
\end{tabular}

* Usual self-care activities include dressing, feeding, bathing, grooming, and toileting. Avocational (recreational and/or leisure) and vocational (work, school, homemaking) activities are patient-desired and age- and sex-specific.

HLA-B27-associated spondyloarthritis with more joints involved than usual (see Chapter 4) and some patients with slightly more severe forms of pauciarticular JRA. However, we mean primarily to include in this group a population whose disease most resembles seropositive early onset adult RA but who do not have rheumatoid factor. Girls are more frequently affected. The ANA test is positive in $25 \%$ of these patients. Rheumatoid nodules are rare. After an average of 7 years of disease, $15 \%$ of these children had entered functional class III or IV as a result of very severe joint destruction. ${ }^{5}$ While the prognosis is not good for the children, it is much better than that of the seropositive group.

\section{Clinical Features}

Onset. By the time they get to the rheumatologist, most of these youngsters have obvious arthritis. However, prior to diagnosis, there is often a period of months during which arthritis is present but not recognized, although the physician knows they are ill with low-grade fever, lethargy, weight loss, and morning stiffness. In addition to symmetrical involvement of many large joints, especially the wrists, these children almost always have distal smalljoint symmetrical arthritis involving the proximal interphalangeal (PIP) and metacarpophalangeal (MCP) joints. Other frequently affected joints include the interphalangeal toe joints, cervical spine, and temporomandibular joints. Synovial outpocketings or pouches are frequently seen overlying the wrists (tenosynovitis) (Fig. 3.6). Joints are frequently warm but usually not tender. Effusions are easily demonstrated, and limitation of extension is apparent in many joints. Occasionally, patients have "dry" arthritis without effusions or warmth but with progressive flexion contractures. If routine pediatric examinations do not include examining the range of motion of joints, the diagnosis may be missed for years. Some of these patients, primarily girls, do not complain of pain despite obvious arthritis. ${ }^{42,246,252}$ 
Rheumatoid Nodules. Subcutaneous nodules near the olecranon process at the elbow are frequently palpable in seropositive arthritic children (Fig. 3.40B). Microscopic examination shows central necrosis and palisading fibroblasts, identical to the pathology in seropositive adult nodules (Fig. 3.21). Polyarticular arthritic children also sometimes have transient nodules over the interphalangeal joints of the fingers and toes. These tend to come and go with exacerbations of the disease. Some authors indicate slight pathologic differences between these nodules and those of adult RA. ${ }^{253,254}$

\section{Course}

Structure of the Joints. Rheumatoid arthritis primarily affects the synovial joints which are diarthroses; that is, a joint resembling a hinge, with a cavity and free movement. ${ }^{255}$ The articulating bones are covered by a hyaline (glasslike) lining, the articular cartilage. The marginal nonarticulating connective tissue is called the synovial membrane (Fig. 3.22). This membrane provides the substances necessary for metabolism of articular cartilage and is the source of synovial fluid.

The joint is surrounded by a fibrous capsule. The bone is lined by a thin connective tissue membrane-the periosteum. Ligaments secure opposing bone surfaces and may attach to bone inside or outside the capsule. Tendons attach muscle to bone inside the capsule. The site of attachment of a ligament or tendon to bone is called an enthesis. The motion of tendons and muscles over bony prominences is facilitated by bursae, closed sacs lined with a protruding bursal (synovial) membrane. There are at least 156 bursae in the body.

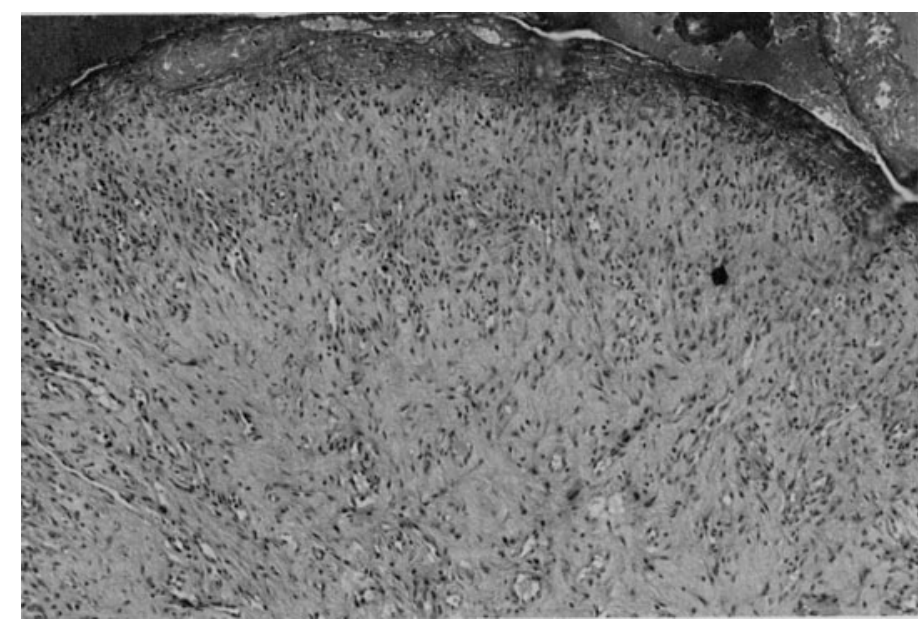

Figure 3.21. Rheumatoid nodule showing typical palisading granuloma. The patient was a 16-year-old boy with cystic fibrosis of the pancreas who developed seropositive rheumatoid arthritis. 


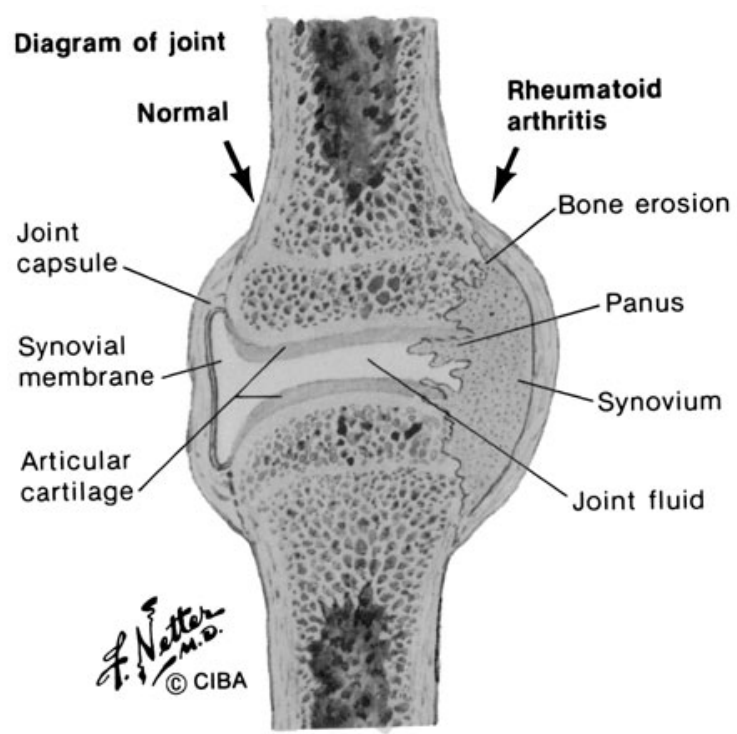

Figure 3.22. Diagrammatic representation of joint in rheumatoid arthritis. (Reprinted with permission from Clinical Symposia 31(4):23, 1979. Copyright by CIBA Laboratories.)

All of these structures may become inflamed and contribute to pathology in and around the joints. ${ }^{254}$ Although the potential exists for varying types of inflammation to constitute different forms of arthritis, sorting out these differences remains to be accomplished. However, the recognition of the importance of the more frequent, severe, and calcifying inflammation at the enthesis in HLA-B27-associated spondyloarthritis provides a clue indicating that immunogenetic techniques may help in identifying different pathophysiologic processes now grouped under one umbrella (JRA or RA) (see Chapter 4).

\section{Pathologic Considerations}

The diverse group of diseases we call JRA, induced by a variety of genetically controlled responses to different environmental stimuli, all have in common inflammation in and around the joint. This inflammation seems to be perpetuated by immunologic events rather than by continued active infection. ${ }^{256}$

Synovium. The inflammatory reaction in the synovium begins with congestion and edema (Table 3.15). This is followed by cellular infiltration, at first with polymorphonuclear leukocytes, then with small lymphocytes, and in advanced cases with plasma cells and multinucleated giant cells. The synovial lining cells multiply, elongate, and palisade. Lymphoid follicles with germinal centers (Allison-Gormley nodules) form in some cases. Blood vessels proliferate and form granulation tissue. The synovium thickens in a villous fashion (Figs. 3.1, 3.22).

Articular Cartilage. In more severe cases, the inflammation extends from the synovium into the articular cartilage. A mantle of granulation tissue, a pan- 
Table 3.15. Synovitis

\begin{tabular}{ll}
\hline \multicolumn{1}{c}{ Characteristics } & Effects \\
Proliferation of synovial lining cells & $\begin{array}{c}\text { Stretching of the capsule, ligaments, ten- } \\
\text { dons }\end{array}$ \\
$\begin{array}{l}\text { Infiltration of synovium by inflammatory } \\
\text { cells }\end{array}$ & $\begin{array}{l}\text { Granulomatous proliferation and villus } \\
\text { formation } \\
\text { Effusion of fluid containing inflamma- } \\
\text { tory cells }\end{array}$ \\
& by pannus and destruction by pro- \\
& teolytic enzymes \\
Increased connective tissue producing \\
stiffness and reduced range of motion \\
Either capsuloligamentous laxity and \\
hypermobility or fibrous ankylosis and \\
ultimately bony ankylosis
\end{tabular}

nus, forms covering the bone and connecting the inflamed cartilage to the inflamed synovium (Fig. 3.22).

Destruction of cartilage by this inflammation leads to bony erosions. If the pannus continues to grow, the cartilage may be continually eroded until it disappears altogether.

Subchondral Bone. Continuous with the inflammation in the synovial tissue is osteitis, inflammation in the bone itself. Periarticular demineralization (osteolysis) is common in epiphyseal bone. If the epiphyseal plate is involved, growth is retarded, and dwarfism may result. Erosions and cystlike areas of destruction may be apparent. Osteoporosis also occurs from disuse.

Periosteum. The synovial recesses in the phalanges are large and extend for long distances along the phalanges. Therefore, synovitis in the proximal interphalangeal joints may be associated with concomitant periostitis in the immediately adjacent bone shaft. Periostitis without synovitis is not characteristic of JRA, although it may be seen in a variety of other disorders including syphilis and HLA-B27-associated spondyloarthritis. Periostitis is rare in JRA.

Ankylosis. Newly formed connective tissue, which constitutes the scar resulting from granulation tissue in and around the joints, may form fibrous contractures and articulations or may rarely even calcify, producing bony ankylosis.

\section{Joint Deformities}

Detailed description of the pathomechanics in each joint are beyond the scope of this monograph. In every joint, a balance exists between the muscles 
and tendons and the bones through which they exert their action. At first, capsular distension and bulging of the synovium may initiate deformity. If the invasive inflammatory synovium grows into the cartilage, tendons, and ligaments, the capsular and ligamentous supports weaken. Inflamed tendons may contract or rupture. Articular surfaces remodel under the forces of abnormal muscle pull. The healthy balance of forces is lost. Soft tissues contract, and there is fibrosis involving the capsule and periarticular tissues, resulting in loss of mobility of the joint. Subluxation may occur in severe disease (Figs. 3.23 and 3.24).

\section{Bone Deformities}

Unique deformities may occur in the growing skeleton. Chronic hyperemia may result in accelerated growth in affected growth centers (Fig. 3.25). An extremity or appendage may be elongated (Figs. 3.26 and 3.27). In the short bones, growth arrest may occur, resulting in permanent underdevelopment of a bone. If accelerated maturity results in asymmetric premature fusion of an epiphysis, the extremity may ultimately be shortened (Fig. 3.40B).

Systemic disease of all sorts inhibits growth, presumably as a result of

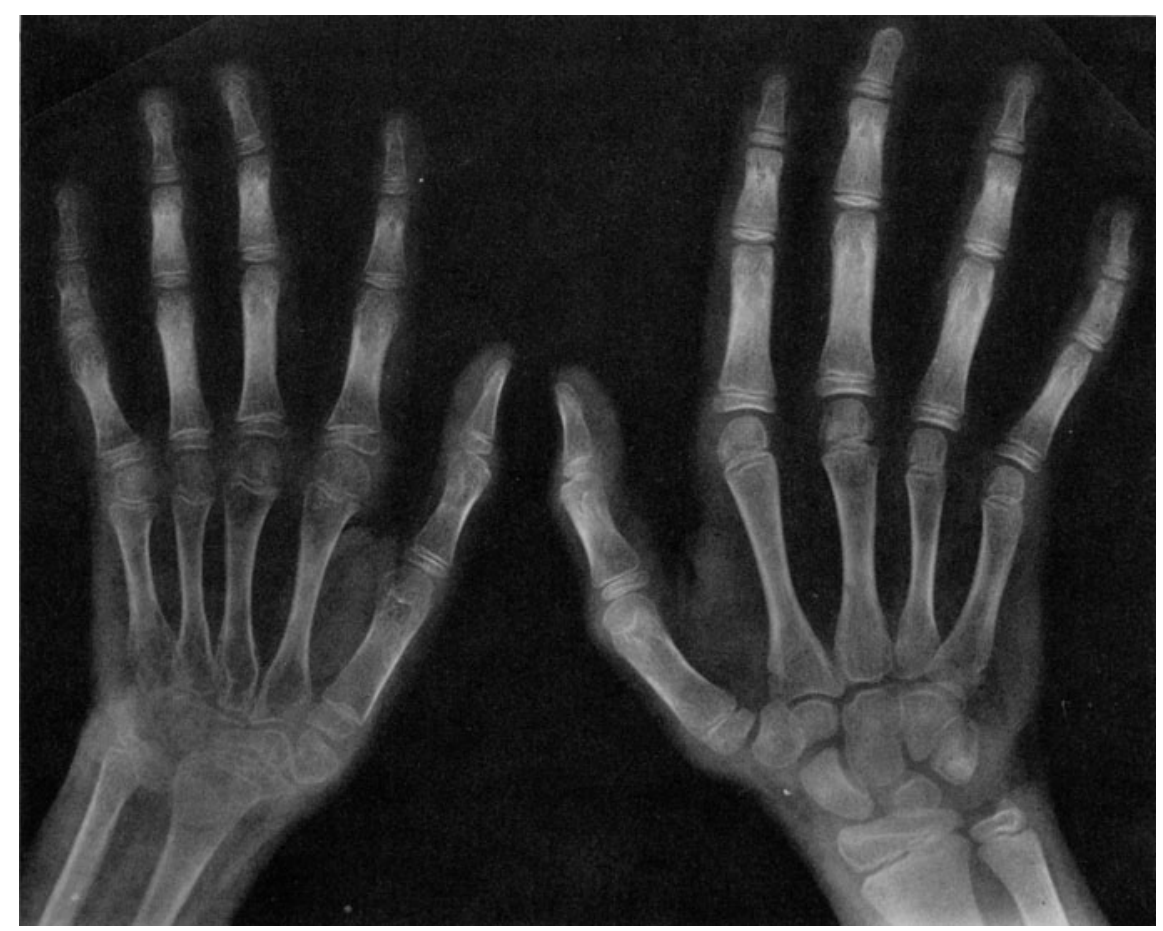

Figure 3.23. Severe arthritis of the left wrist in a 9-year-old girl with onset of pauciarticular seronegative, B27-negative arthritis at age 2 years. 


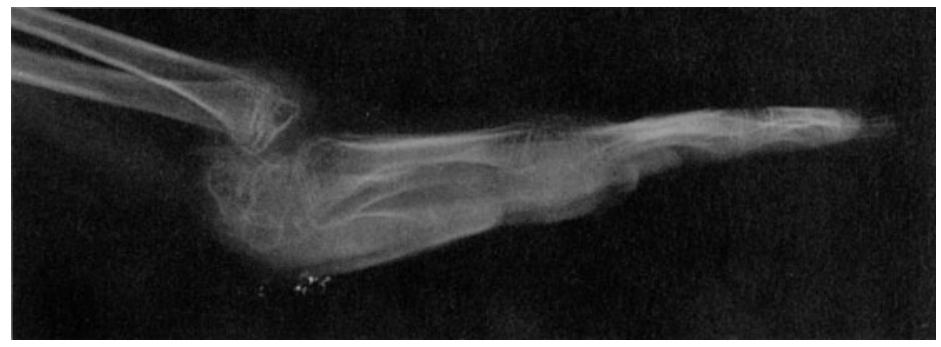

Figure 3.24. Lateral view of wrist shown in Fig. 3.23. This child, with minimal disease elsewhere, has "disappearing bones" (the "opera-glass hand") with severe subluxation.

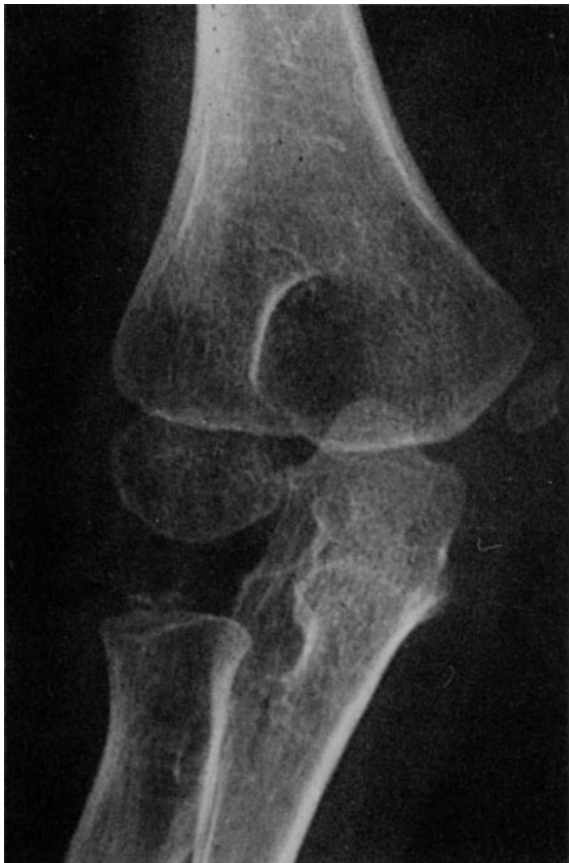

A

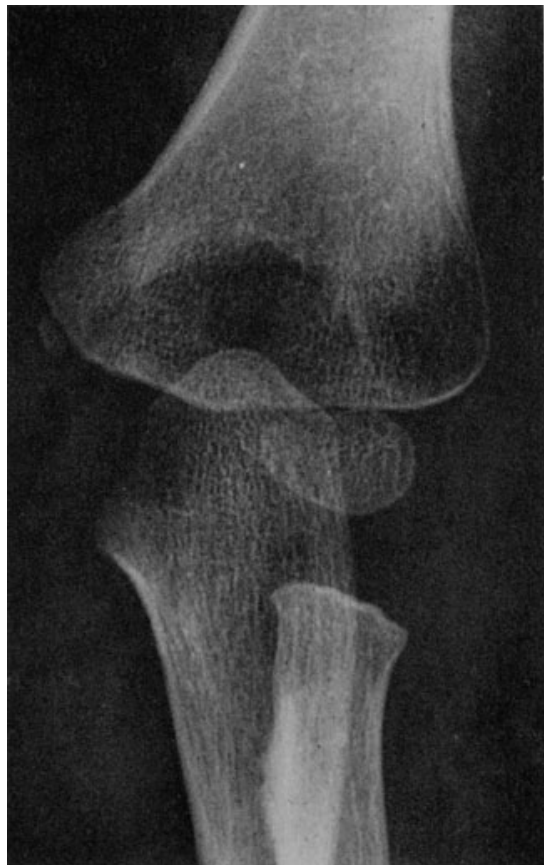

B

Figure 3.25. Growth disturbance in JRA with advanced skeletal maturation on the involved side and retarded maturation on the opposite side. Patient is a female, aged 3 years 9 months. Involved elbow (A) shows advanced skeletal maturation, 5 years, in involved areas. Uninvolved elbow (B) shows retardation in skeletal maturation, 2 years 8 months, in corresponding areas without local disease. (Reprinted with permission from Grokoest et al., ref. 42.)

end-organ inability to respond to growth hormone. Inflammation at the growth plate and deformities may also contribute to short stature. The administration of daily corticosteroids also arrests growth, presumably by a suppressive effect on cell proliferation in peripheral tissues. Large doses of 


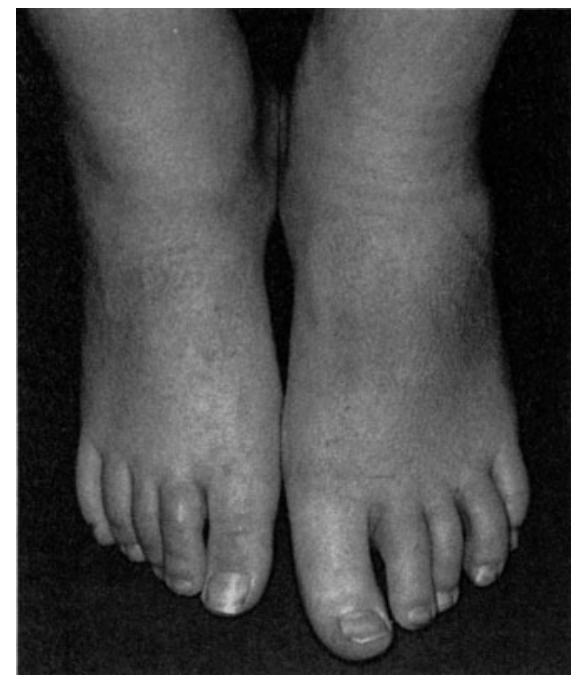

Figure 3.26. Enlargement and accelerated growth of the left foot in JRA.

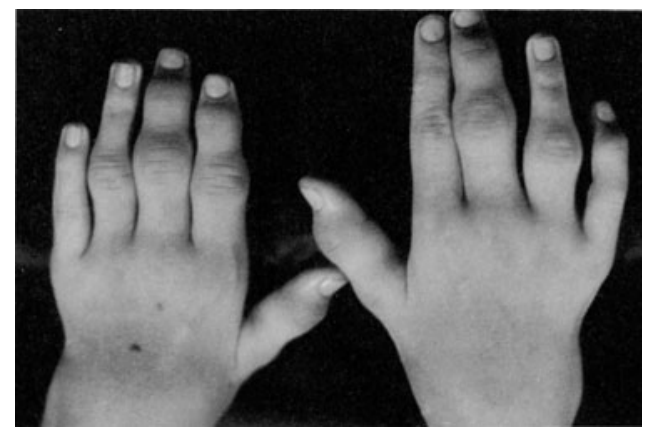

Figure 3.27. The right second finger has linear overgrowth, the left fifth finger is foreshortened.

alternate-morning steroids ( $>1 \mathrm{mg} / \mathrm{kg} /$ day) may also stunt growth, whereas small doses may accelerate growth by controlling systemic disease.

Bone erosions are a late finding in JRA, resemble those of adult RA, and are first seen on intra-articular bone surfaces unprotected by cartilage (Fig. 3.28). Joint narrowing and fibrous and bony ankylosis are seen in severe cases (Fig. 3.23).

\section{Specific Joint Involvement}

Temporomandibular Joint. Temporomandibular joint (TMJ) disease is common in all forms of childhood arthritis but most severe in girls with progressive disease. ${ }^{257}$ It may be asymptomatic and overlooked on routine examination. Interference with the normal growth pattern in the mandible results in a shortened ramus. In rare patients, the mandibular dysplasia is asymmetric. 


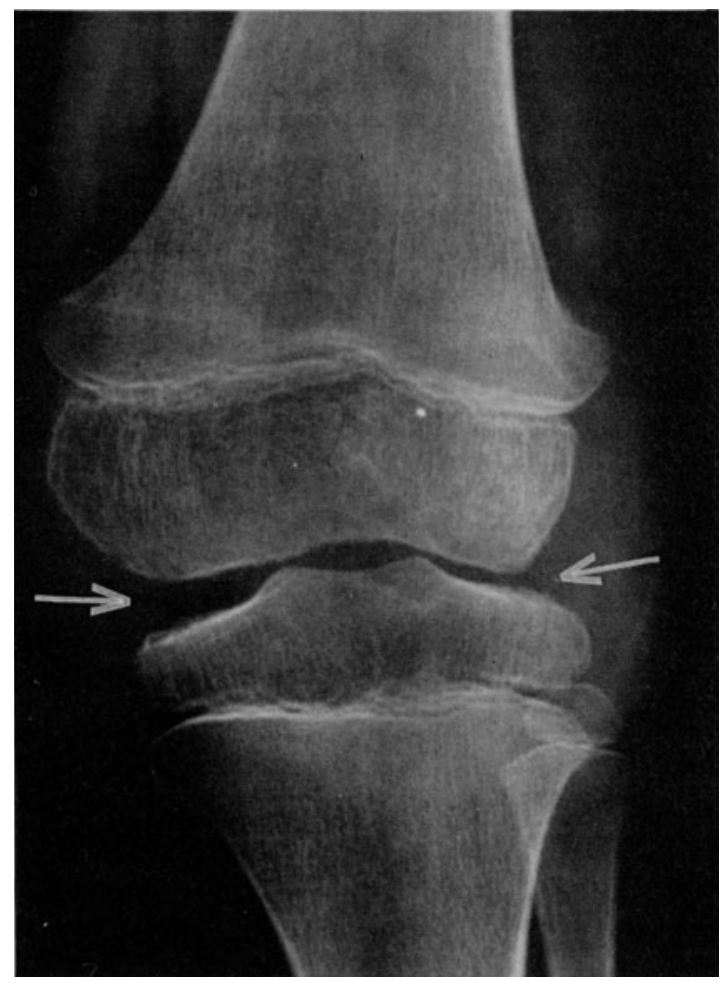

Figure 3.28. Knee in severe systemic polyarticular JRA (age 7 years; onset at age 3 years) showing erosions and narrowed joint space (arrow) (see also Fig. 3. 44).

The soft-tissue profile of the face is determined by the triad of nose, lips, and chin. Although the chin is only a part of the mandible, it is the most conspicuous frontal component in man. A pleasing and harmonious profile is often lost in JRA (Fig. 3.29A). ${ }^{42,258}$

The micrognathia of JRA is also often associated with gross dental malocclusion. There may be difficulty in chewing food, and the patient may speak with a lisp. Only the second molar teeth may contact when the patient closes her mouth. Tendinous insertions of the lateral pterygoid muscles may be destroyed along with the condyle, which is flattened, eroded, and rarefied. The mouth is then opened as if it were a hinge, with little or no gliding motion.

Elongation osteotomy of the body of the mandible and restoration of the contour of the jaw by iliac bone grafting or silicone implant may result in great cosmetic improvement ${ }^{259}$ and contribute to the psychological wellbeing of the arthritic teenager (Fig. 3.29B). In rare instances, the interincisal opening decreases to a dangerously small orifice due to ankylosis of the TMJ. A vitallium plate may then be inserted to restore joint motion to normal.

Cervical Spine. Cervical spine involvement is common in all subsets and torticollis may be the sole presenting symptom of childhood arthritis. Loss 


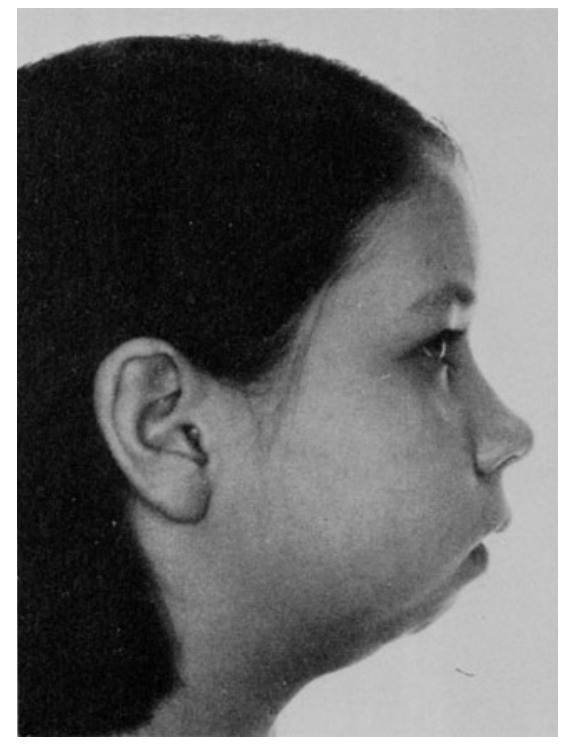

A

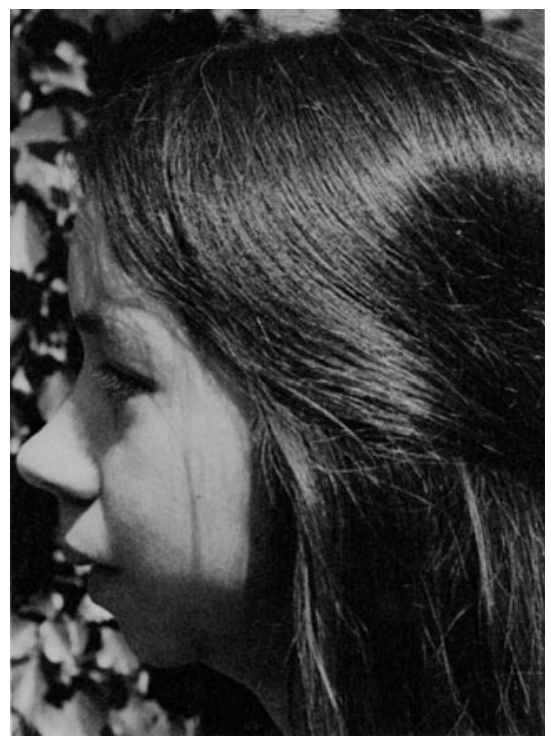

B

Figure 3.29. (A) Mandibular dysplasia (HLA-B27-associated spondyloarthritis) also shown in polyarticular JRA in Fig. 3.41. (B) Bone-graft reconstruction of the chin produced an obvious change in affect.

of the sharp margins of the articular facets of the C-2-C-3 apophyseal joint may first be seen radiologically after years of clinical disease (see Fig. $4.28) .{ }^{42,43,260}$ If the disease progresses, erosions occur, followed in even more severe cases by bony bridging and fusion of the cervical segments. Intervertebral disk spaces may be narrowed and the stature and anterior-posterior diameter of the vertebral bodies may. be diminished.

Growth deformities in the cervical spine result in a short neck with increased cervical lordosis, dorsal kyphosis, and compensatory overgrowth of the lumbar vertebrae (Figs. 3.20 and 3.30). ${ }^{260}$ Although cervical spine involvement almost always starts at the C-2-C-3 level, it may occasionally extend down to the thoracic spine (Figs. 3.31 and 3.32). ${ }^{42,43}$ Subluxation of the atlas on the axis, as is seen in severe adult RA, may occur in the worst cases but rarely causes a clinical problem. ${ }^{261,262}$ Spinal-cord compression may rather occur in the thoracic area as a complication of steroid-induced epidural lipomatosis. ${ }^{263}$

Anesthesiologists must be aware of potential difficulty with intubation and of the additional hazards (dislocation, fracture, and cord injuries) that may occur during anesthesia. ${ }^{264}$ In patients who develop laryngospasm and cannot be intubated tracheostomy may be necessary. ${ }^{259}$ Spinal or local anesthesia should be used whenever possible. ${ }^{265}$ 


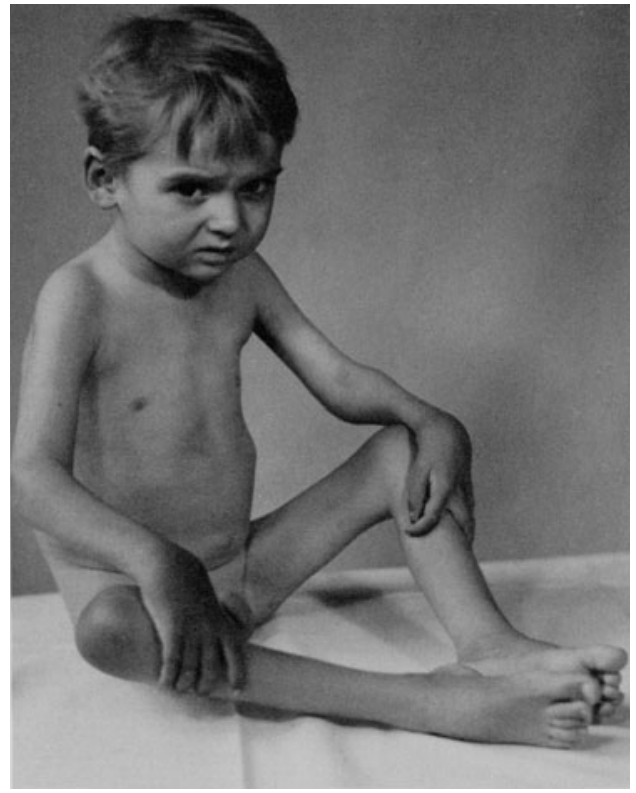

Figure 3.30. Growth deformities in the cervical spine result in a short neck with increased cervical lordosis and dorsal kyphosis (see also Fig. 3.20).

Cricoarytenoid Arthritis. Schlesinger first called attention to laryngeal arthritis causing stridor, dyspnea, and cyanosis in JRA. Occasionally, lifethreatening stridor is the presenting manifestation of systemic-onset JRA. ${ }^{78,114}$ Tracheostomy may be avoided by prompt institution of prednisone therapy, which can usually subsequently be weaned away after adequate salicylates have been administered. Some cases may respond to periodic oral beclomethasone diproprionate (BDP) inhalation. ${ }^{266}$ The acute swelling that produces the dramatic initial symptom subsides without apparent significant residua. Hoarseness may result from chronic laryngeal arthritis in polyarticular patients. Laryngeal ankylosis has not been reported in childhood, but fibrosis can make intubation for surgery difficult in older children. ${ }^{264}$

Peripheral Joint Manifestations. A detailed description of the combined results of inflammation and destruction of each individual joint of the growing child is available elsewhere ${ }^{268}$ and is beyond the scope of this monograph. Articular and periarticular pain and swelling are followed promptly by juxtaarticular demineralization. Muscular wasting is soon apparent in affected areas. Overgrowth of the epiphyses may produce gross enlargement of the rapidly developing ends of the long bones, while the shafts may be thinned. Small bones may be underdeveloped. Specific radiographic and clinical findings may include: ${ }^{42,43,268}$ 


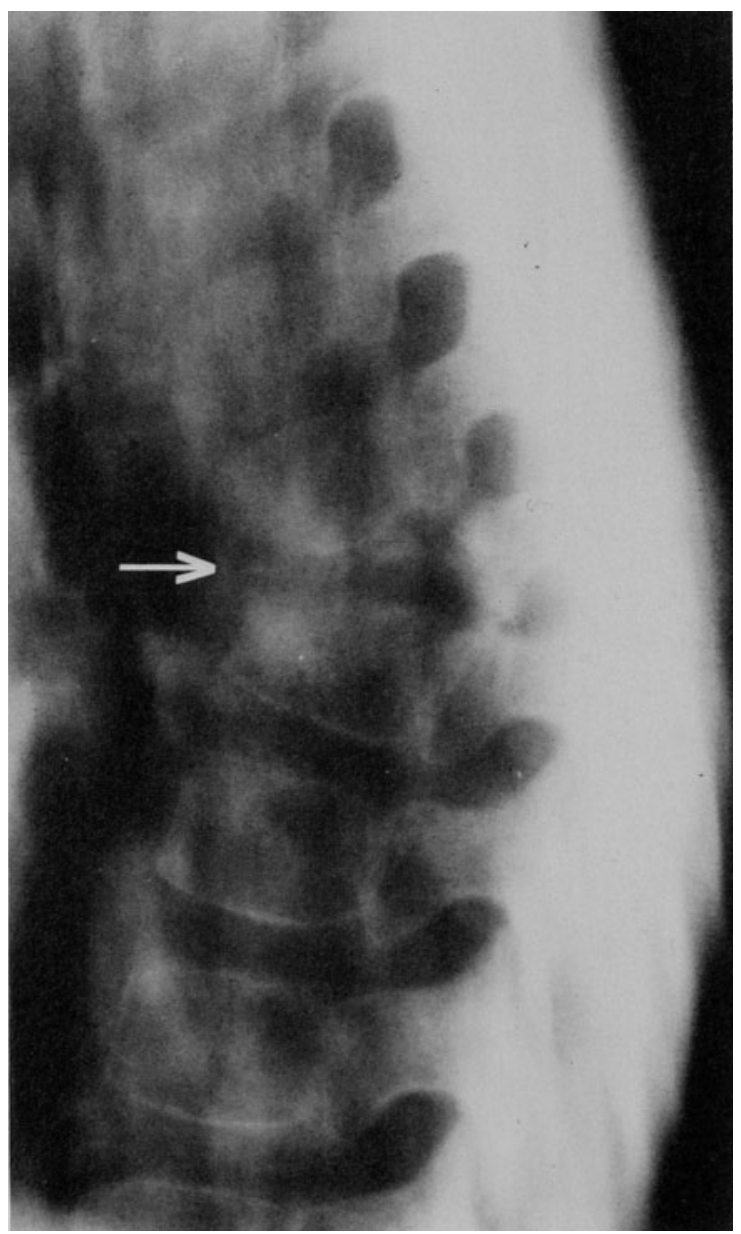

Figure 3.31. Complete destruction of mid-dorsal vertebra with gibbus deformity resembling tuberculosis in a 9-yearold boy with severe systemic polyarticular JRA. Needle biopsy demonstrated "rheumatoid inflammation" (Fig. 3.32). Vertebral destruction by rheumatoid inflammation is rarely reported. ${ }^{267}$

Hands and Wrists. ${ }^{269}$ Altered length and width of digits and individual bones, crenation, crowding and fusion of carpal and carpometacarpal joints and bones into solid masses, proximal interphalangeal joint contractures and fusion, and spindle and pencil-tip appearance of the fingers. In the hand and wrist, there is normally a complicated balance between the forces of a multitude of tendons and ligaments. As this is lost, collapse deformities occur with hyperextension of one joint and compensatory flexion of another. Classic deformities include "swan neck" and "boutonnière" deformities of the fingers, palmar subluxation, and radial or ulnar deviation of the MCP joints (Fig. 3.33). Use of the hand in activities of daily living may accentuate the deformity, and a vicious cycle of deforming forces may be established. 


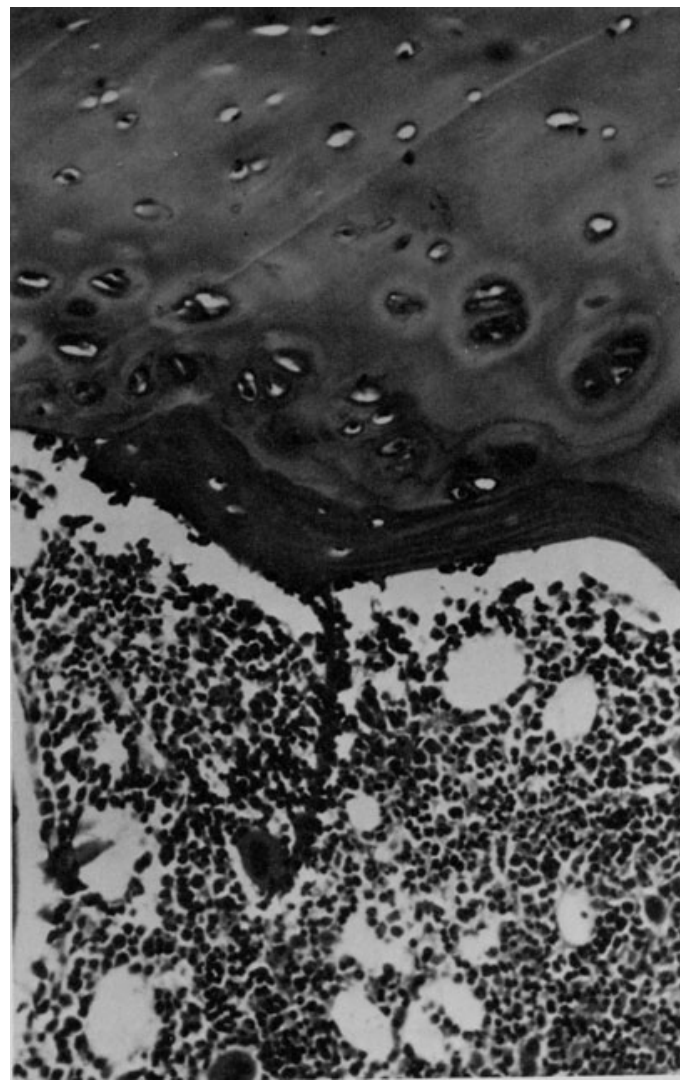

A

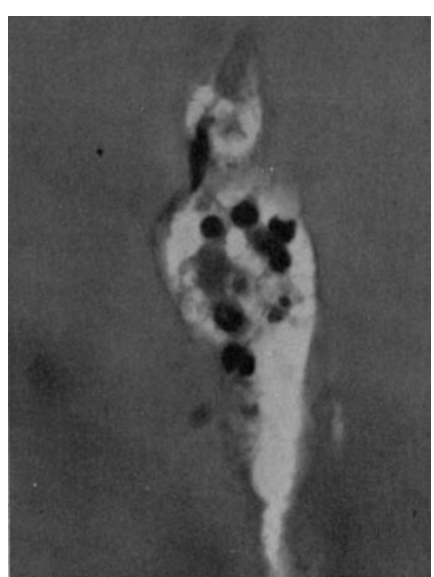

B

Figure 3.32. Needle biopsy of the apophyseal joint ( $T-7)$ in patient (Fig. 3.31) showing chondromucinous changes in the deeper layers of articular cartilage (A), causing destruction (hyperplastic bone marrow below); in a highpower view (B) lymphocytes can be seen invading a bone lacuna in articular cartilage. $($ A $) \times 40$; (B) $\times 128$.

Knees. Flexion contracture at the knee is common (Fig. 3.34). In addition to all of the usual manifestations of arthritic inflammation at the knee, children often develop an impressive valgus deformity. This may result from overgrowth of the leg at the knee, requiring the child to assume the valgus position to walk with less limp (Fig. 3.35).270 However, it may also result from hip disease when limitation of external rotation of the hip causes assumption of the valgus position at the knee. Since we have adopted a policy of providing both lifts to the short leg and vigorous physiotherapy aimed at correcting limited hip rotation, we have not had to do stapling procedures and only in one case osteotomies to correct the knee valgus.

Hips. Hip involvement is common in all forms of childhood arthritis and is characterized by both a flexion contracture and by simultaneous limitation of full flexion, abduction, and rotation (Fig. 3.34). Some of the findings are a result of iliopsoas and adductor spasm. The hip pathology is sometimes 


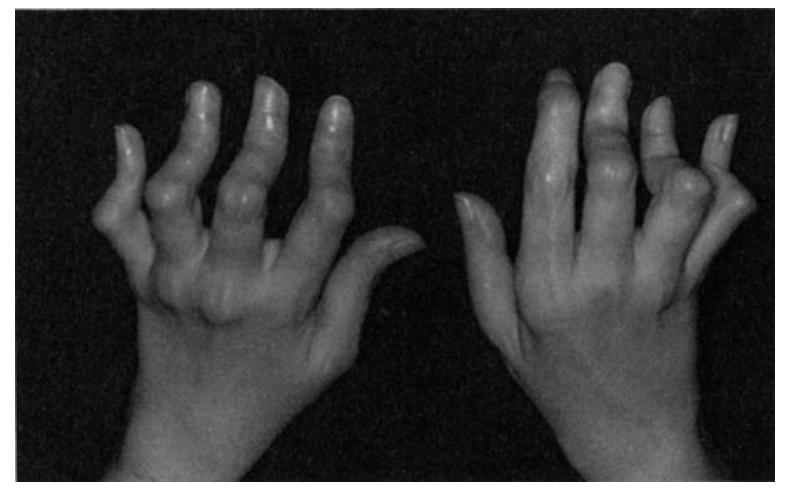

Figure 3.33. "Boutonnière" deformities (left, $2-5$; right 3-5) chacterized by flexion deformities of the proximal interphalangeal joints and extension deformities of the distal interphalangeal joint. "Swan-neck" deformities consisting of hyperextension of the proximal interphalangeal joint and flexion of the distal interphalangeal joint are also developing in the right second finger. Patient had the onset of polyarticular seropositive JRA at age 9, without multisystem disease but with positive antinuclear antibodies, low serum total hemolytic complement, and elevated anti-DNA antibodies. Now age 32, she continues to have destructive arthritis as her only symptom. The deformities resemble those seen in the "lupus hand" but are not correctable and are accompanied by radiologic evidence of severe destructive changes. More common finger abnormalities in severe long-standing JRA are shown in Figure 3.27

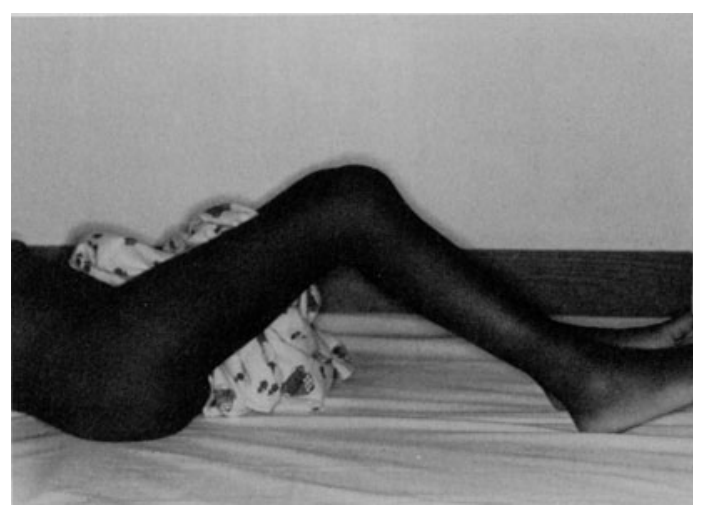

Figure 3.34. Hip and knee contractures typical of the child allowed to become wheelchairbound; the child sits all day with the knees and hips flexed. Rehabilitation requires surgical release of iliotibial bands and other soft-tissue contractures about the hips and knees with a vigorous immediate postoperative exercise program and prohibition of the wheelchair.

masked by a typical compensatory increased lumbar lordosis, which, if noted, provides a clue to its existence (Figs. 3.6, 3.41).

There is loss of substance and broadening of the femoral head. The neck is often poorly developed, and cystic changes appear in the head and neck. Later, marked narrowing of the joint space may be accompanied by secondary degenerative changes and aseptic necrosis of the femoral head. Acetabular destruction and protrusio, coxa magna, and dislocation may occur (Figs. 3.36, 3.51, 3.54, and 3.56). 


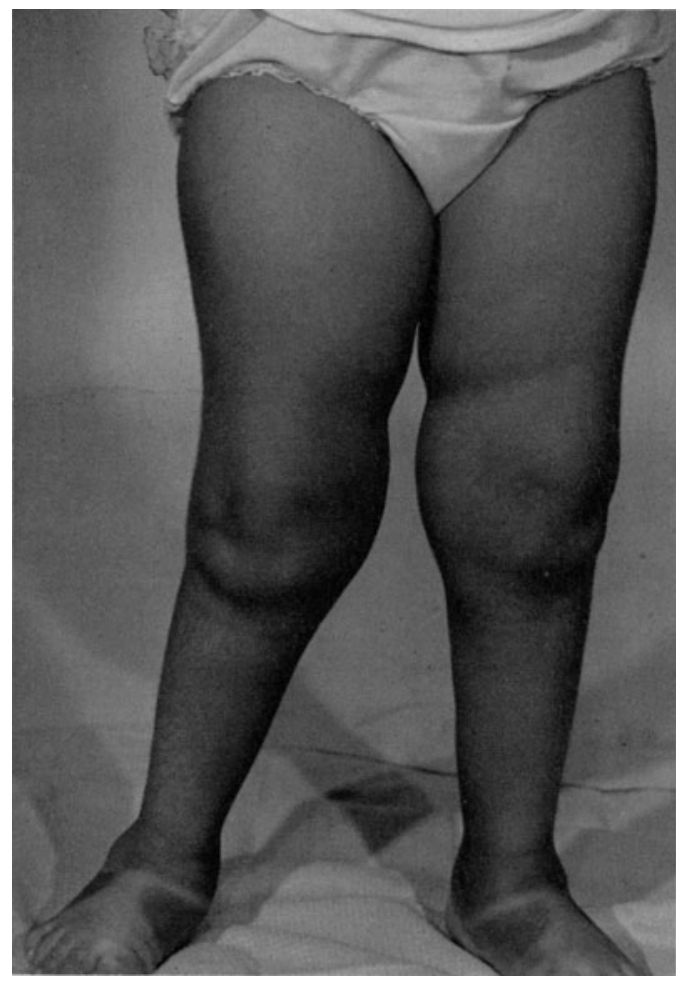

Figure 3.35. Although the patient has bilateral knee (and ankle) effusions, the right leg has overgrown, resulting in the child's having to stand with the knee in valgus; the deformity is progressive. Surgical correction (stapling or osteotomy) is avoided by lifting the opposite (good) heel by building up the oppposite shoe. This deformlty is also caused by nip disease resulting in limited external rotation of the hip with compensatory assumption of the valgus knee posture to walk; this may be treated with physical therapy to maximize external rotation at the diseased hip.

Feet. Although the knee is the most frequently involved joint in all forms of childhood arthritis, significant scarring is more frequent at the ankle and tarsus (Fig. 3.37). The foot is a complex articulation involving many joints in several planes. In addition to the many synovial joints, many bursae and tendon sheaths may be diseased, just as in the hand (Fig. 3.38). Fibrous, cartilaginous, or bony fusion of tarsal bones (tarsal coalition) may be mistaken for a congenital anomaly. Displacement of the talus by effusion may simulate "vertical talus" (Fig. 3.39) and be the presenting manifestation of JRA.

Deformities of the hips and knees may also cause compensatory deformities in the feet. Valgus deformity is more common than varus, but both may occur. Valgus foot deformities are sometimes compensatory for valgus knee deformities.

Elbows. Limitation of supination of the elbow is one of the most common and subtle diagnostic signs of childhood arthritis and may occur without flexion contracture. Most severely affected arthritic children have limitation of both extension and supination (Fig. 3.40). 
290 3. Juvenile Rheumatoid Arthritis

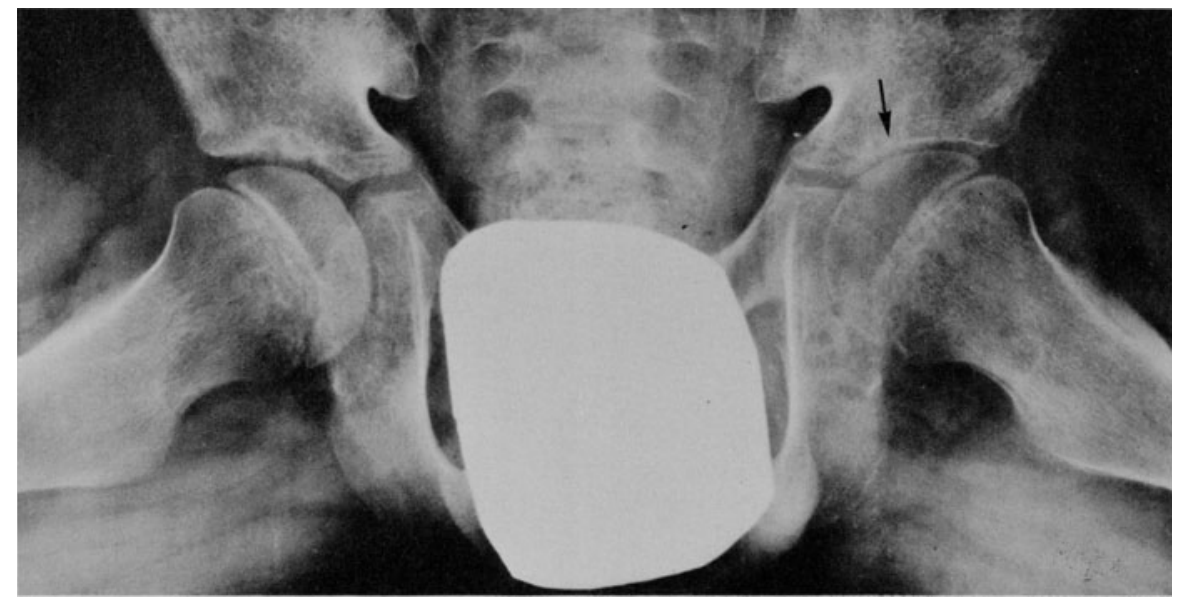

A

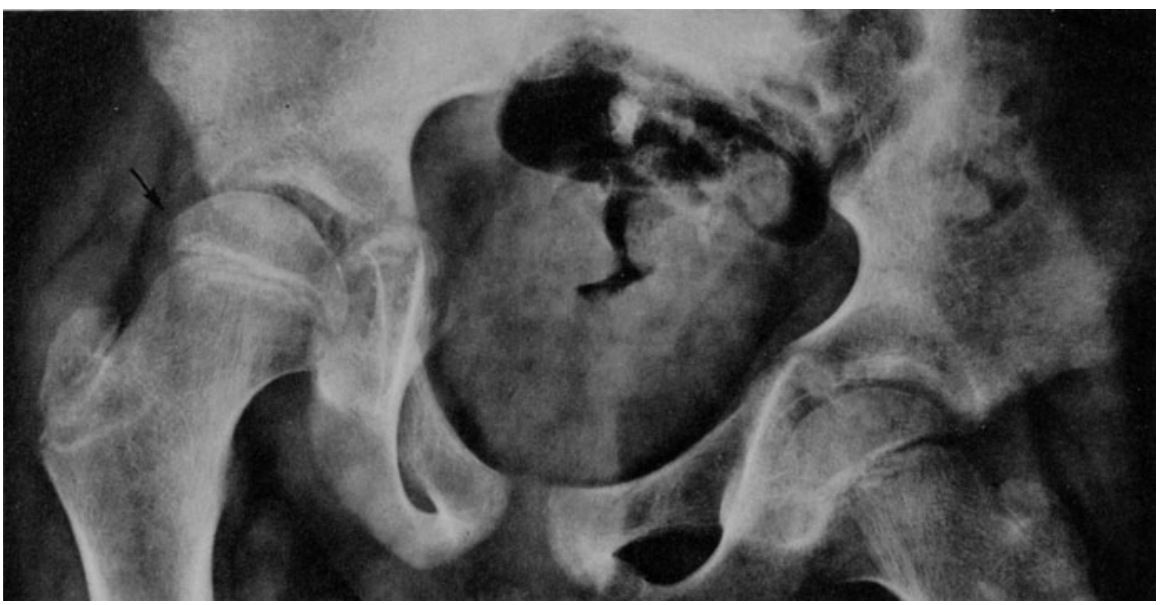

B

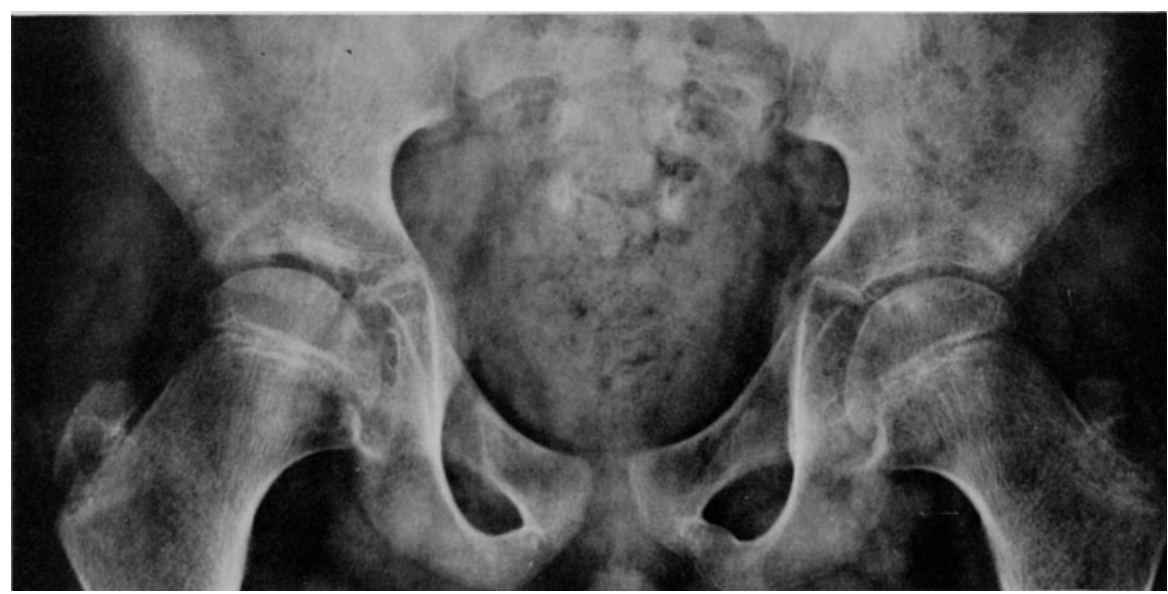

C 

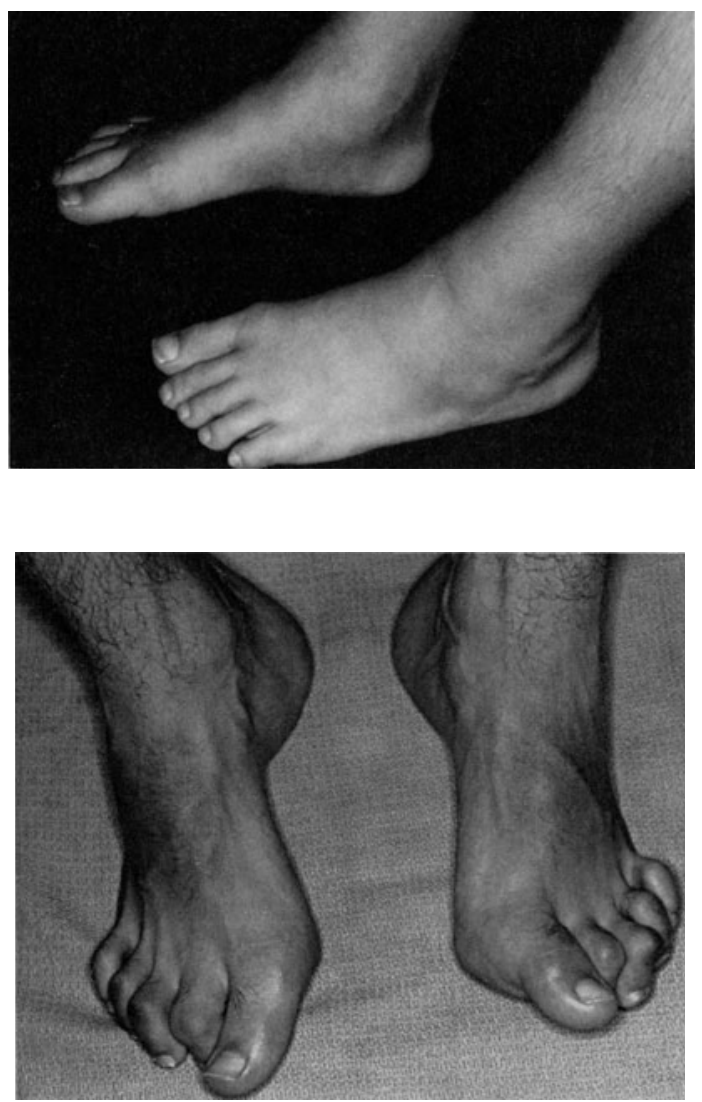

Figure 3.37. Inability to dorsiflex the ankles and limited eversion are common findings in polyarticular JRA.
Figure 3.38. Hallux valgus and flexion contractures of the toes in a teenage boy with polyarticular JRA. The toe abnormalities are the same as those seen in the fingers. Cavus feet and/or bunions may be the presenting manifestations of childhood arthritis.

Shoulders. The shoulders are frequently affected in severe polyarticular disease. Both the acromioclavicular joint and the glenohumeral joint may be affected; the latter may be accompanied by necrosis of the humeral head and growth disturbances in the humerus. Severe disease may produce painful swelling of the joint and all the surrounding bursae (Fig. 3.40).

Figure 3.36. Bilateral hip disease in an 8-year-old boy with systemic JRA (onset at age 3). (A) Following a jump into the swimming pool, there was acute chondrolysis of the left hip, illustrated here by sudden joint-space narrowing (arrow) not seen previously (see Chapter 2, Acute Chondrolysis of the Hip, and Fig. 2.56). (B) Six weeks later joint-space restoration is apparent in the left hip (similar to that seen in patients without arthritis who suffer acute lamellar coxitis, Fig. 2.56) but the right hip is seen to be subluxed, apparently as a compensation for the painful left hip. Without treatment (other than continuing aspirin), the subluxation disappeared on the right side, and the joint space in the left hip returned to normal (C). The patient has continued to have severe arthritis and, following a flare of systemic manifestations at age 15, had accelerated bilateral destruction of the hip joints that frequired surgical replacement at age 16 . 
292 3. Juvenile Rheumatoid Arthritis

A
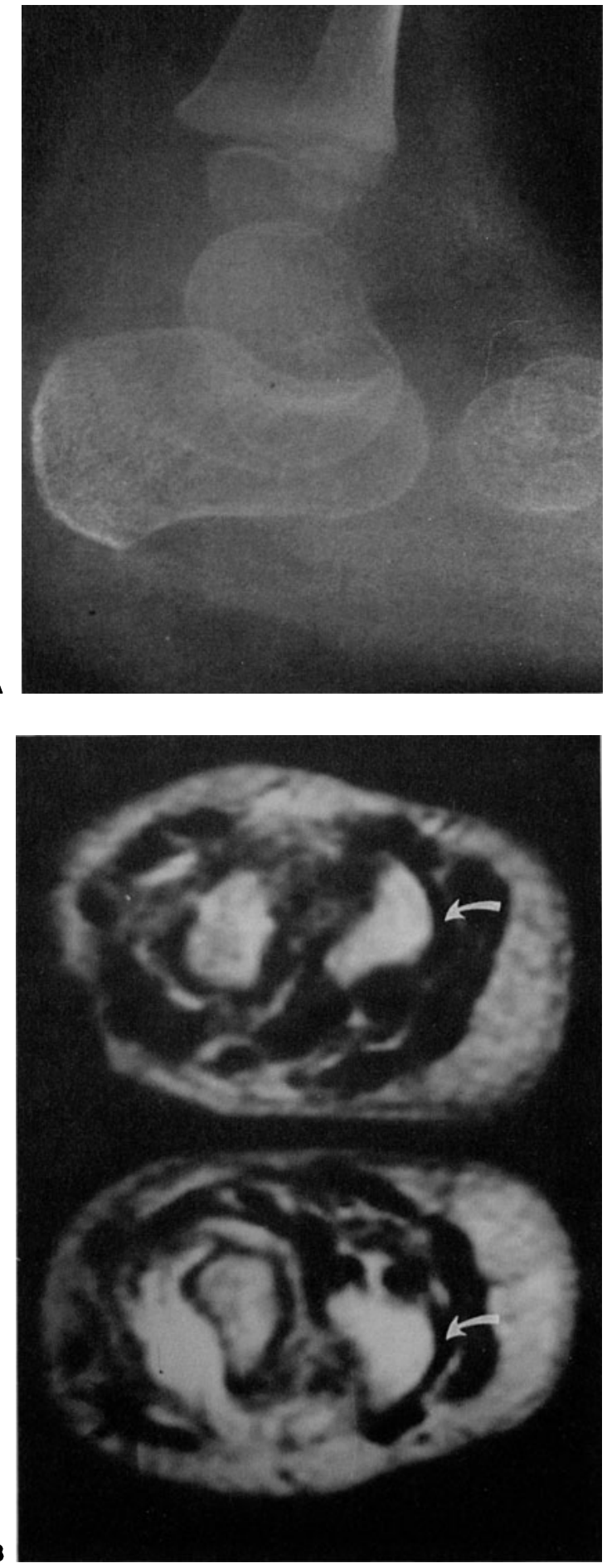

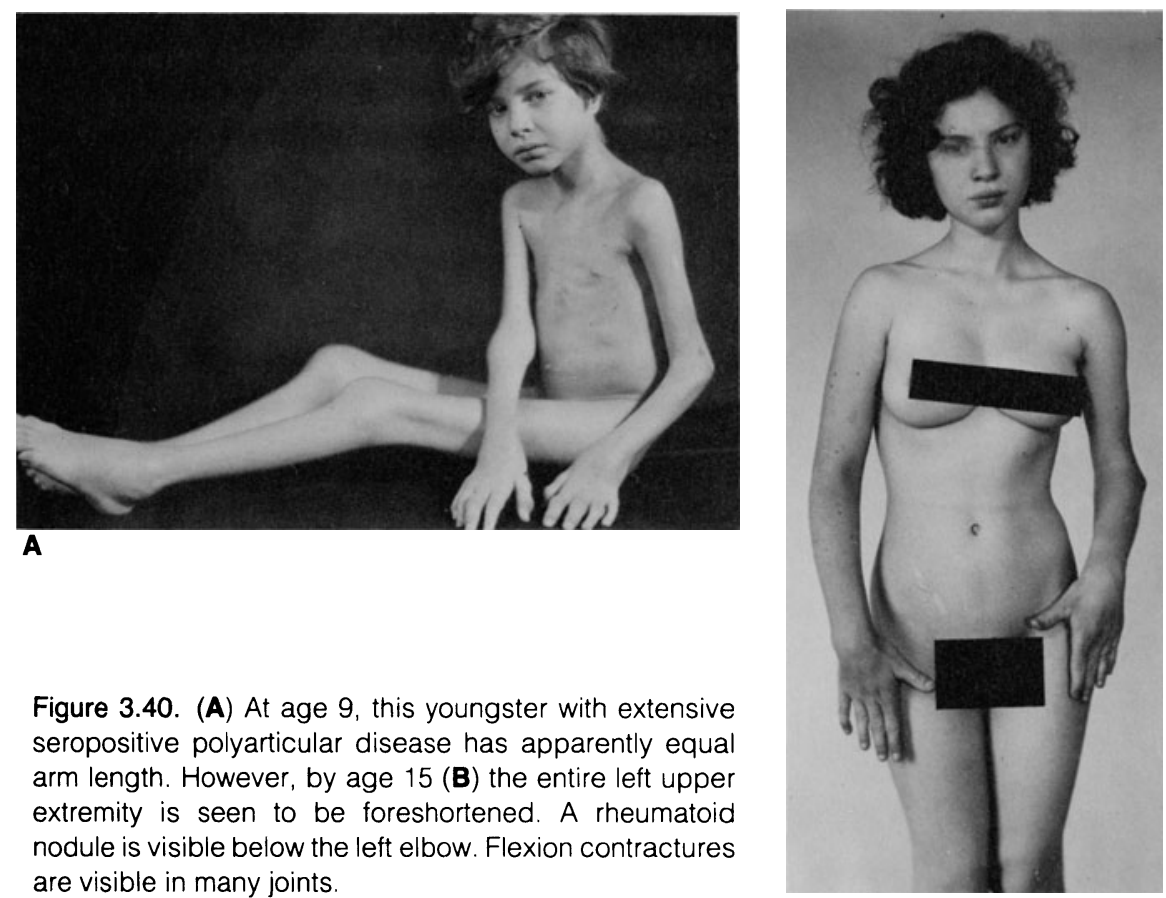

Figure 3.40. (A) At age 9, this youngster with extensive seropositive polyarticular disease has apparently equal arm length. However, by age 15 (B) the entire left upper extremity is seen to be foreshortened. A rheumatoid nodule is visible below the left elbow. Flexion contractures are visible in many joints.

B

\section{Treatment of Polyarticular JRA}

Drugs are only one part of the prescription for children with rheumatic disease. Drugs are important, however, and talented prescribing indicates knowledge and competence. Physical and occupational therapy help to maintain strength and function. The attitudes of the prescriber and the therapeutic team may determine compliance, maintain morale of the child and family, and enable growth and development to proceed despite the soulsapping nature of chronic sickness. The physician and the patient/parent become partners in control of the disease. A sense of control, albeit less control than one would wish, is ego rewarding and helps combat depression. Part of prescribing for these children involves teaching the parent and eventually the child the purposes of each medication, its potential benefits and side effects, and the methods used to regulate the dose so as to achieve maximum benefit and minimum side effects. The maximum potential benefit from drugs can only be achieved with such teamwork (Fig. 3.41).

Figure 3.39. (A) JRA presenting as ankle effusion displacing talus to simulate vertical talus. (B) MRI performed for evaluation of apparent vertical talus shows effusions (arrows) displacing talus. Correct interpretation avoids surgery. 


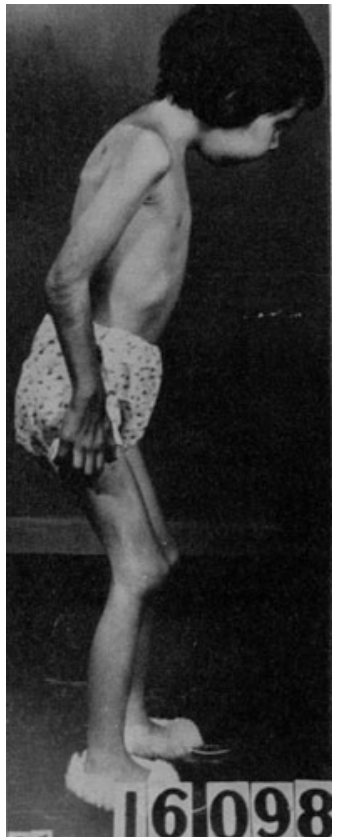

A

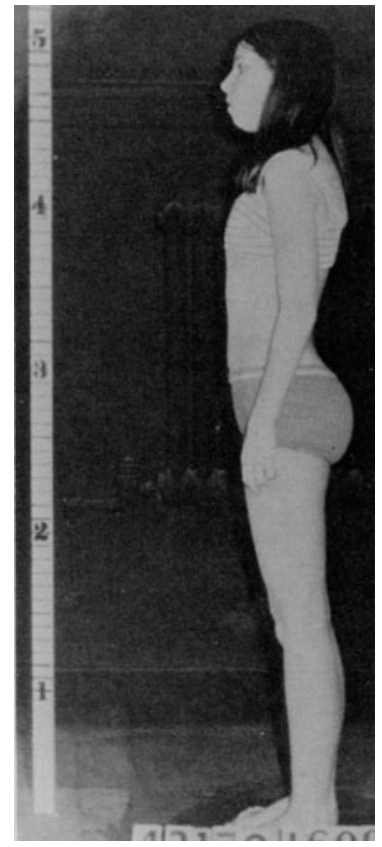

B
Figure 3.41. Example of the accomplishments of a therapeutic team in the care of a child with severe polyarticular JRA. At age 6, both hips were said to be dislocated (A). Nine years later, although she still had severe arthritis with delayed puberty and short stature, she could function in normal teenage society (B). (Reprinted from the Arthritis Reporter, Spring 1972, with permission of the Arthritis Foundation, New York Chapter, and Dr. J.S. Stillman.)

\section{Anti-inflammatory Drugs}

Salicylates. Aspirin is no longer the primary therapeutic agent in JRA even though it is generally equally effective and much cheaper than any currently available, equally safe alternative therapy. ${ }^{271}$ Most parents and some doctors fear aspirin and choose the risks of other NSAIDs in preference to aspirin. ${ }^{272}$ As a result we now have extensive experience with the newer nonsteroidal anti-inflammatory agents in children. The principles and techniques of therapy have already been discussed in the section on systemic JRA.

Other Nonsteroidal Anti-inflammatory Drugs (NSAIDs). The salicylate radical has been chemically modified to produce an ever-increasing number of agents that have as their goal better efficacy and fewer side effects (Table 3.16). ${ }^{273}$ The average daily cost is $10-15$ times that of ordinary aspirin. More than 50 antirheumatic compounds are reported to be currently under investigation. None have been proved to retard the rate of cartilage destruction in humans ${ }^{274}$ or to induce remission of disease. So far, no one agent has proved to be superior for all patients. ${ }^{271,273}$ In general, however, indomethacin, tolmetin, naproxen, and sulindac appear to be more effective in HLA-B27associated spondyloarthritis. ${ }^{271,275,276}$ Agents with a long half-life such as naproxen, which requires administration only twice daily, achieve better 
Table 3.16. Drugs Used for the Treatment of Arthritis in Children

Nonsteroidal Anti-inflammatory Agents: Salicylate Preparations

Aspirin, 325-mg or 81-mg tablets

Enteric-coated aspirin, 325-mg, 500-mg, 975-mg tablets

Sustained-release aspirin, 650-mg tablets

Choline magnesium trisalicylate, 1 tsp $=500-\mathrm{mg}$ equivalent, $500-750-\mathrm{mg}$ and 1000-mg tablets

Salsalate (salicylsalicylic acid), 500-mg, 750-mg tablets

Nonsalicylate NSAIDs Labeled by U.S. FDA for Use in Children

Tolmetin (Tolectin), 20-30 mg/kg/day (4); 1800-mg max dose

Naproxen (Naprosyn), 10-15 mg/kg/day (2); 1500-mg max dose

Ibuprofen (Motrin), 40-50 mg/kg/day (4); 2400-mg max dose

NSAIDs Not Yet Labeled by U.S. FDA for Use in Children

Indomethacin (Indocin), ${ }^{*} 1-3 \mathrm{mg} / \mathrm{kg} / \mathrm{day}(3-4) ; 200-\mathrm{mg}$ max dose

Fenoprofen (Nalfon), 40-50 mg/kg/day (4); 3200-mg max dose

Ketoprofen (Orudis), 3-5 mg/kg/day (4); 300-mg max dose

Meclofenamate (Meclomen), ${ }^{\dagger} 4-7 \mathrm{mg} / \mathrm{kg} /$ day (3); 300-mg max dose

Sulindac (Clinoril), 4-6 mg/kg/day (2); 400-mg max dose

Flurbiprofen (Ansaid), 3-4 mg/kg/day, (3-4), 300-mg max dose

Piroxicam (Feldene), $0.3 \mathrm{mg} / \mathrm{kg} /$ day single daily dose

Diclofenac (Voltaren), 2-3 mg/kg/day (3-4), 200-mg max dose

Diflunisal (Dolobid), 7-21 mg/kg/day (2-3), 1500-mg max dose

\section{Slower-Acting Antirheumatic Drugs}

Hydroxychloroquine, $7 \mathrm{mg} / \mathrm{kg} /$ day; 300-mg max dose

Gold (injectable), 0.5-1 mg/kg/week; $50-\mathrm{mg}$ max dose

Gold (oral), $0.1 \mathrm{mg} / \mathrm{kg} /$ day; 9 -mg max dose

Penicillamine, $5-10 \mathrm{mg} / \mathrm{kg} / \mathrm{day}$; 500-mg max dose

Immunosuppressive agents

Methotrexate, $10 \mathrm{mg} / \mathrm{mm}^{2} /$ once weekly

* Labeled for use in resistant cases.

† Preliminary trials in children showed excessive toxicity.

Numbers in parentheses indicate usual number of daily doses.

compliance and may be more effective on this basis alone in all forms of arthritis. ${ }^{238,277}$ Objective comparisons of efficacy and tolerance of all these agents and their comparison to aspirin and the other salicylates have been handicapped by the general lack of availability of serum levels. Therapeutic trials have been conducted with arbitrary dosage, making no allowance for differences in individual absorption, metabolism, and excretion. ${ }^{196}$ Pharmacokinetic principles, which had led to improvement in the care of asthmatic and epileptic children, have not yet been applied to the use of these agents in children. ${ }^{278}$ 
A paucity of clinics with sufficient numbers of patients and staff to conduct controlled clinical trials has also delayed the accumulation of knowledge about these drugs in children. Most tests with adults have included primarily seropositive patients; the number of children to whom the conclusions are directly applicable is small. The few reported studies in children have made no effort to separately determine efficacy among the various subsets of childhood arthritis. ${ }^{226}$ In the United States, at the time of this writing, only three of these agents (tolmetin, ibuprofen, and naproxen) have been tested sufficiently to satisfy the requirements of the Food and Drug Administration (FDA) for labeling for general use in arthritic children. This dilemma is not easily solved. On the one hand, it is well established that the results of drug studies in adults should not be applied to children without testing in children. On the other hand, some sick children are being deprived of medications that would undoubtedly be of benefit to them and are available to adults with identical conditions. Both the children and the drugs are thus "orphaned."

Availability of these agents has led to a new approach in our management of older arthritic children. We often have the youngster try a number of different agents briefly. Within a few days, many youngsters can report their individual preference based on subjective relief of symptoms and tolerance of side effects (Table 3.17). Maximum benefit, however, is not seen in some children until after 12 weeks of administration of relatively high doses. ${ }^{279}$

Tolmetin Sodium. The U.S. JRA Cooperative Drug Study Group demonstrated, within the limitations stated above, that tolmetin sodium, 20-30 mg/ $\mathrm{kg} /$ day in three or four divided doses, was equal or better than aspirin, 80$100 \mathrm{mg} / \mathrm{kg} /$ day in four doses, in terms of anti-inflammatory effect and was slightly better tolerated than aspirin. ${ }^{226}$ Studies suggest a half-life in adults of 4-6 $\mathrm{h}$ and peak serum levels 40 min after single doses. ${ }^{280}$ In adult nonarticular rheumatism and soft-tissue disease, common in pauciarticular JRA, tolmetin surpassed aspirin in efficacy. We find it very useful in spondyloarthritic children. In the pediatric clinical trial, liver enzyme abnormalities related to aspirin therapy promptly returned to normal. ${ }^{226}$ Licensing of the drug in the United States includes children, so informed consent and therapeutic trial protocols are not required, and the drug may be used in children by general physicians. It is available only in bad-tasting $200-\mathrm{mg}$ tablets that must be divided and disguised for children who cannot swallow tablets. It is about 10 times as expensive as ordinary aspirin but only twice as expensive as flavored soluble "baby" aspirin tablets administered in similarly efficacious dosage.

Naproxen. Although not tested in the United States, experience in Europe showed sufficient efficacy and tolerance in children to warrant FDA labeling of pills and syrup for children in the United States. ${ }^{281-283}$ A long half-life of $13 \mathrm{~h}$ has been confirmed in children, providing greatly increased con- 
Table 3.17. Adverse Reactions Associated with the Use of Nonsteroidal Antiinflammatory Drugs.

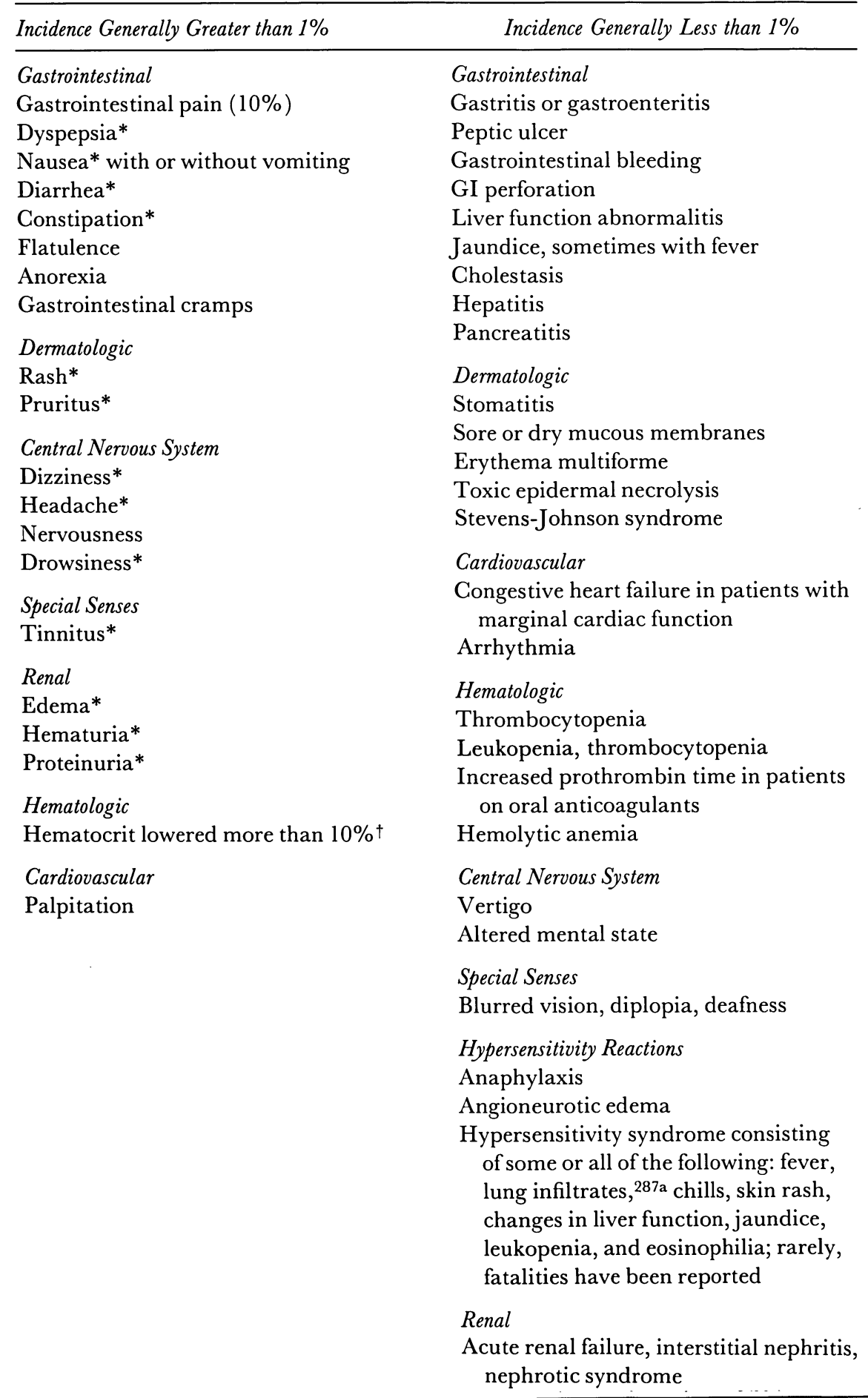

\footnotetext{
* Incidence generally between 3 and $9 \%$. (Those reactions occurring in less than $3 \%$ of patients are unmarked.)

† Incidence generally $42 \%$ in children.
} 
venience, better compliance, and perhaps improved control of disease. In adults with RA, preference was expressed for naproxen and other antiinflammatory drugs compared with aspirin, but this effect was entirely explained by better compliance. ${ }^{238}$ However, in ankylosing spondylitis (AS), naproxen was a considerably better agent than aspirin and was even slightly preferred over indomethacin, the agent previously found to be more effective in AS. ${ }^{238}$ I have found it generally well tolerated and an excellent agent for use in teenagers with spondyloarthritis, both HLA-B27-positive and -negative. ${ }^{275} \mathrm{In}$ small children, it has been tested at $10-15 \mathrm{mg} / \mathrm{kg} /$ day in two divided doses. ${ }^{281-285}$ We use the larger dose and find it to be well tolerated. In teenagers, we generally start with $250 \mathrm{mg}$ twice daily and increase the evening dose to 375 or $500 \mathrm{mg}$ as needed and tolerated. Some children cannot tolerate naproxen due to lethargy or psychic effects ("like being on a trip"), and one youngster who was greatly benefited could not tolerate the palpatations it occasionally produces. Bullous photosensitive rashes are frequent in those with "little Orphan Annie" complexion. ${ }^{286,287}$

Ibuprofen. Ibuprofen, $20 \mathrm{mg} / \mathrm{kg} / \mathrm{day}$, has been shown to be safe and efficacious in children, but the half-life is short and divided doses are necessary. ${ }^{288}$ The drug is extremely well tolerated, is available as a syrup, and has sometimes been helpful in children who could not tolerate other drugs (Fig. 3.42). However, liver enzyme abnormalities seen with aspirin therapy may or may not return to normal with ibuprofen. Higher doses are generally required for control of fever in children with systemic JRA ( $40 \mathrm{mg} / \mathrm{kg} /$ day).

Indomethacin. Indomethacin, $2 \mathrm{mg} / \mathrm{kg} / \mathrm{day}$ in four divided doses, has been used safely in children with control of fever and arthritis. ${ }^{188}$ There is great variability in half-life; twice-a-day dosage, advocated in adults, has not been reported in children. This drug was better than aspirin in most trials involving patients with AS and related disorders. ${ }^{238,239}$ However, naproxen is preferred by many patients who cannot tolerate the headaches, dizziness, and fatigue caused by indomethacin. Higher-than-recommended doses have been reported in association with death from liver disease and infection in children ${ }^{290}$ and aplastic anemia seems to be more frequently associated with indomethacin than other NSAIDS. ${ }^{291}$ The drug seems reasonably safe in proper dosage and is, in some patients with HLA-B27-associated spondyloarthritis, preferred to all others. Oral suspension is available; indomethacin is FDA labeled for use in children resistant to alternative NSAIDS.

Other NSAIDs not FDA labeled for use in children but studied by the Pediatric Rheumatology Collaborative Drug Study Group and found safe and efficacious include fenoprofen $(40 \mathrm{mg} / \mathrm{kg} / \mathrm{day}), 292$ furbiprofen $(4 \mathrm{mg} / \mathrm{kg} /$ day, ${ }^{293}$ ketoprofen $\left(4 \mathrm{mg} / \mathrm{kg} /\right.$ day), ${ }^{294}$ proquazone $(20 \mathrm{mg} / \mathrm{kg} /$ day $),{ }^{295}$ meclofenamate $\left(7.5 \mathrm{mg} / \mathrm{kg} /\right.$ day), ${ }^{296}$ and pirprofen $\left(15 \mathrm{mg} / \mathrm{kg} /\right.$ day) ${ }^{297}$ European studies report safety and efficacy of piroxicam $(0.3 \mathrm{mg} / \mathrm{kg} /$ day in a single dose $),{ }^{284}$ sulindac $\left(2.5 \mathrm{mg} / \mathrm{kg} / \mathrm{day}\right.$ in two doses), ${ }^{298}$ and diclofenac $(2-3 \mathrm{mg} / \mathrm{kg} /$ 


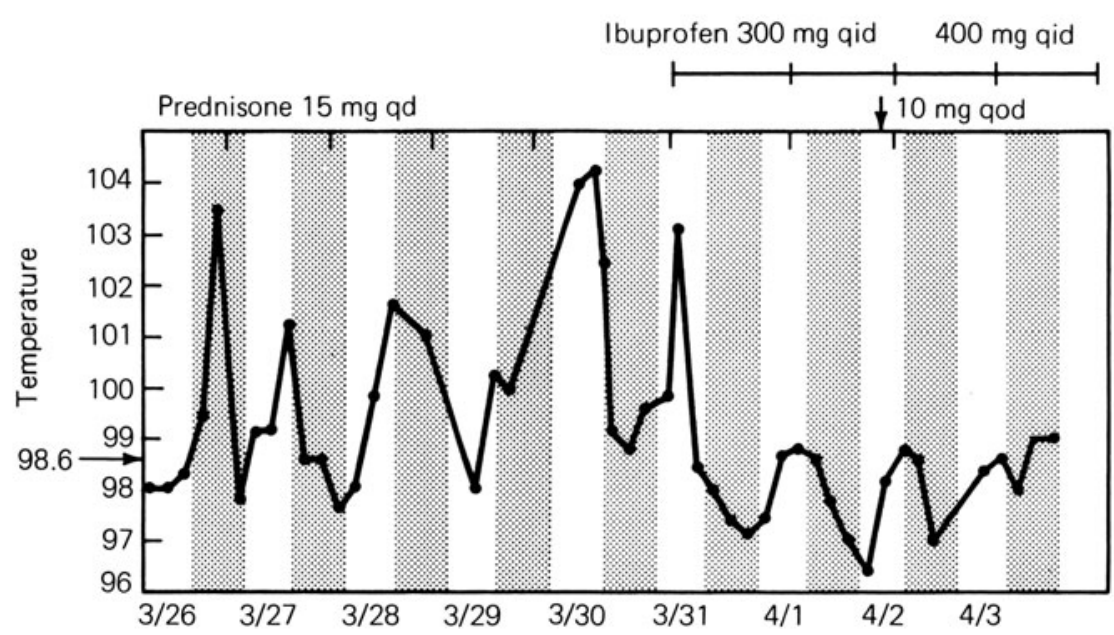

Figure 3.42. Steroid-sparing effect of NSAIDs. Nonsteroidal anti-inflammatory drugs, such as ibuprofen, may be used to control fever in children with systemic JRA who are unable to tolerate salicylates. NSAIDs may be used together with alternate-day steroids in patients whose fever cannot be controlled with NSAIDs alone. Alternate-day prednisone plus an NSAID may be more effective in controlling fever than daily prednisone alone, as shown in this illustration. (Reprinted with permission from Brewer EJ: Nonsteroidal antiinflammatory agents. Arthritis Rheum 20:513-525, 1977; copyright by American Rheumatism Association.)

day). ${ }^{285,299}$ It is unclear whether the risk of hepatotoxicity is greater with diclofenac than with other NSAIDs. ${ }^{300}$ Rash from meclofenamate is so frequent that it is probably a poor choice for children. ${ }^{296}$ Phenylbutazone may cause death from aplastic anemia and is not used in children. ${ }^{291,301}$

NSAIDs may result in disappearance of phenotypically activated circulating $\mathrm{T}$ cells and functionally activated $\mathrm{B}$ cells along with improvement in ESR and rheumatoid factor in responsive patients. ${ }^{302}$ Absorption may be delayed but is not ultimately impaired by simultaneous administration of sucralfate an agent which we frequently administer to children with GI intolerance of NSAIDs, which often relieves that problem. ${ }^{303} \mathrm{H}_{2}$ blocking agents may reduce blood levels of NSAIDs. ${ }^{304}$ Efficacy and safety of misoprostol in children have not been established. ${ }^{305,306}$ Fortunately children not exposed to alcohol and tobacco are at lower risk of serious GI bleeding than are adults.

Studies of interactions between NSAIDs have produced conflicting results. ${ }^{271,307}$ To avoid interference and a lower net effect, only one antiinflammatory drug is generally used at one time in any patient. In a few special circumstances, the combination of aspirin and another NSAID such as indomethacin or naproxen seems to have been helpful to our patients. However, such combinations may increase the risk of analgesic nephropathy. ${ }^{130}$ 
Hazards of NSAIDs. These new drugs have all of the potential toxic side effects of aspirin ${ }^{271}$ plus a higher incidence of anaphylactic-type reactions. ${ }^{307 a, b}$ Our early experience suggested that extraordinarily high doses of indomethacin might be associated with increased susceptibility to infection, but this has not been a problem with more appropriate doses. ${ }^{290}$ The major symptoms limiting use of these drugs in children are gastrointestinal intolerance ${ }^{308-310 b}$ and central-nervous-system manifestations, including headache, drowsiness, depression, and depersonalization reactions. These reactions may occur after taking a single tablet. ${ }^{311}$ Other worrisome problems with these agents include the reported reduction in renal function, the potential to cause interstitial nephritis and renal papillary necrosis (analgesic nephropathy), and the occasional occurrence of the nephrotic syndrome, interstitial nephritis and irreversible renal failure. ${ }^{130,271,312-324}$ Medication should be stopped $48 \mathrm{~h}$ prior to surgery which has a risk of hemorrhage. ${ }^{324 a, b}$ Children are at less risk than adults for renal complications. ${ }^{325}$

Slower-Acting Antirheumatic Drugs. Sulfasalazine, gold, chloroquine, and penicillamine, the drugs included in this category, do not have the standard laboratory properties of anti-inflammatory drugs and generally are rather specific for rheumatic diseases. Weeks to months of therapy are required before a beneficial effect may be expected. While it is possible they may in some cases induce remission of the disease and inhibit cartilage destruction, they have not been proved to do so. ${ }^{326-331}$ They are generally not effective or tolerated during the years of systemic illness in systemic JRA; ${ }^{332}$ injectable gold and sulfasalazine have been associated with life-threatening systemic reactions.

The indication for use of the slower-acting drugs is generally considered to be persistently poorly controlled polyarticular disease that threatens multiple joint destruction and accumulating disability (Fig. 3.16). Potential therapeutic benefits must be balanced against the potentially serious side effects of treatment with these agents. The number of children requiring these medications is small, their potential toxicity is great, and considerable clinical experience is necessary for their safe and effective use. The care of such children should be either in or supervised by pediatric rheumatology clinics.

Sulfasalazine. This combination of sulfapyridine and 5 aminosalicylic acid, long used in children with inflammatory bowel disease, has recently become a favored second-line agent for both children and adults with arthritis. ${ }^{333-344}$ Very little of the salicylate and almost all of the sulfa are absorbed. ${ }^{338}$ The mechanisms of action are unknown but seem to be antibacterial and antiinflammatory. In our experience a dose of $30 \mathrm{mg} / \mathrm{kg} /$ day is well tolerated by most children and produces add-on or better benefit in terms of reduction in effusions, joint contractures, and number of affected joints in $60 \%$ of arthritic children on NSAIDs who can continue this medication for 3 months. ${ }^{337}$ Evi- 
dence of efficacy is confirmed by return of ESR to normal within 3 months in $80 \%$ of those children in whom it was elevated. Good results were seen in all forms of childhood arthritis except systemic JRA patients who in the preliminary trial did not tolerate it. ${ }^{337,342-344}$ Within 3 months the medication had to be stopped for allergic rashes/serum sickness (12\%), GI complaints $(6 \%)$, or neutropenia $(4 \%)$ in almost one-quarter of the children; a pair of twins who responded well subsequently had to stop the medication because of pronounced and persistent elevations of SGOT/PT partially caused by insecticide spray, but then definitely worsened by sulfasalazine.

Although double-blind randomized trials of efficacy have not yet been carried out in children, studies in adults suggest that sulfasalazine is more efficacious than hydroxychloroquine and equal to parenteral gold in preventing erosions at 1 year provided that the agent was started early in the course of the illness. ${ }^{334,335} \mathrm{~A}$ recent comparative review found little difference in efficacy between methotrexate, injectable gold, penicillamine, and sulfasalazine, with sulfasalazine most efficatious in lowering ESR. ${ }^{345}$

In addition to the well-recognized gastrointestinal, hematologic, and allergic complications, most of which occur during the first month or 3 months of therapy, rare pulmonary, hepatic, cardiac, and neurologic side effects may occur, and drug-induced lupus may also occur..$^{346-351}$ Nevertheless, the risk/ benefit ratio favors sulfasalazine over other second-line agents and we now offer it to any non-systemic JRA patient whose arthritis is not completely controlled with NSAIDs.

Chrysotherapy. We no longer administer parenteral gold to children. ${ }^{352}$ Gold evolved as a therapeutic agent for RA as a result of Koch's demonstration that the in vitro growth of tubercle bacilli was inhibited by gold and Forestier's erroneous assumption that tuberculosis was related to RA. Despite a multitude of studies, the mechanism of action of gold in RA remains unknown. ${ }^{353}$

Chrysotherapy was in wide use for 30 years before the controlled study of the Empire Rheumatism Council managed to demonstrate that it was more effective than placebo; the difference in effect was not overwhelming, and the advantage of 20 weeks of parenteral gold therapy was lost at the time of 2-year follow-up..$^{354,355}$ No similar study has been performed in children. However, many experienced physicians have reported the efficacy of gold in some pediatric cases and the need for continued maintenance therapy to maintain the clinical effect. ${ }^{199,356}$ Scientific studies do not prove the commonly stated clinical dictum that gold has the capability of inducing a remission. Naturally, in any disease characterized by exacerbations and remissions, any long-term therapy will coincide on occasion with remission.

Fifteen to thirty percent of children started on parenteral gold therapy must discontinue treatment because of adverse side effects. ${ }^{357}$ Gold salts are reported to be 10 times more toxic than any other therapy used in Great Britain and over a 7 -year period accounted for 16 deaths in the U.K. ${ }^{358,358 a}$ 
Death is most commonly from narrow aplasia and its complications, especially overwhelming sepsis. Other side effects include severe mucocutaneous reactions, autoimmune thrombocytopenia, and membranous nephropathy. We have seen one child with fatal disseminated intravascular coagulation and reviewed the record of another (not fatal) after the second test-dose injection of gold and are now aware of many other similar cases (Fig. 3.43). ${ }^{169,170,178}$

Some toxic reactions to gold seem to be immunologically mediated. For example, proteinuria is 32 times more common in patients with HLA-DR3 and so seems to be genetically controlled. ${ }^{359}$

When the decision to give parenteral gold is made, a complete blood count, platelet count, blood chemistry determination, and urinalysis are obtained, and a test dose of gold is given as soon as the results are available. The total weekly dose is calculated as $0.5-1 \mathrm{mg} / \mathrm{kg} / \mathrm{dose}$ (50-mg maximum for adults). ${ }^{199}$ Patients are started with a test dose equal to one-fifth the weekly dose which is increased one-fifth weekly so that the full dose is achieved on the fifth injection. A complete blood count, platelet count, and urinalysis are obtained on the day of each dose, and the gold is not given until the results are checked by the physician. The injection is omitted if there is any rash or laboratory abnormality. Unexplained fever early in the course of gold therapy suggests the possibility of sepsis due to neutropenia and requires immediate evaluation. Serum gold levels are generally unavailable and have not yet been proved to be useful.

To avoid long waits in the clinic, we taught responsible parents to give the intramuscular injections at home after our telephone approval indicating that the laboratory studies that day were normal. ${ }^{360}$ With this system, they had the blood and urine studies in their own neighborhoods, and some of the inconvenience of gold injections was removed. So long as the child was improving, we continued the gold weekly, usually for a period of 1 year. If the

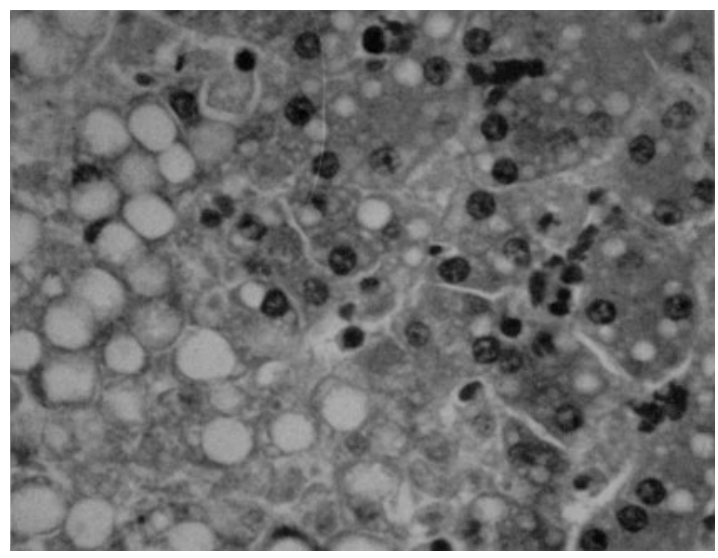

Figure 3.43. Disseminated intravascular coagulation (clogging blood vessels with red blood cells) with necrosis and fatty metamorphosis of the liver following the second injection of gold. 
youngster was in remission, we gradually reduced the gold injections to every 2, 3, and then 4 weeks and, if still in remission, discontinued the injections after about 2 years. If there was no improvement with gold, we gave up the therapy after 6-9 months. If the arthritis worsened soon after reducing the frequency of gold administration, we resumed the weekly injections. A number of different intramuscular gold preparations are marketed. There are no clear-cut distinctions between them. Most of the injected gold is excreted via the kidneys and some goes through the gastrointestinal tract. ${ }^{361,362}$ However, with time, there are increasing deposits in all tissues, which remain for years after cessation of therapy. A controlled trial showed oral gold to be safe and modestly efficacious in children. ${ }^{363}$ Injectable gold is probably only marginally, if at all, better than oral second-line agents in children. ${ }^{345,363}$

Oral Gold (Auranofin). In a well-conducted multi-institutional collaborative study comparing placebo with auranofin over a 6 -month period there was only marginal $(p=0.24)$ benefit of oral gold over placebo at 6 months. ${ }^{364}$ There is some suggestion that early treated patients do better than children first treated after 2 years. Although oral gold has the potential to cause all of the same side effects as parenteral gold they seem to occur in lower frequency. ${ }^{365,366}$ Diarrhea limits the dose usually to $0.15 \mathrm{mg} / \mathrm{kg} /$ day with a maximum of $9 \mathrm{mg}$ daily. Careful monitoring for adverse side effects is carried out monthly. Steady-state blood levels are achieved after 3 months of therapy. ${ }^{367}$ Although only about $13 \%$ of children treated with oral gold for years are still taking it after 5 years, even though $83 \%$ of that series still had signs of active arthritis, ${ }^{368}$ possible efficacy and reasonable safety may make trials of auranofin worthwhile in severely affected children whose disease is not controlled by NSAIDs and sulfasalazine. ${ }^{369}$

Penicillamine. Penicillamine was initially found to be of use in Wilson's disease and has been used in lead poisoning and cystinuria in children. The discovery that penicillamine helps some adults with RA resulted from a trial by Jaffe in a patient with high-titer rheumatoid factor. ${ }^{370}$ Based on laboratory evidence that the drug could dissociate human macroglobulins in vitro, he hypothesized that penicillamine would cause intravascular dissociation of IgM rheumatoid factor. The patient apparently benefited, and the level of rheumatoid factor was reduced, but for unknown reasons. Despite a great many studies, the mechanism leading to improvement remains unknown. Unlike gold, whose efficacy can be demonstrated in experimental models of arthritis such as adjuvant disease, penicillamine is ineffective in any such model. About all that can be said is that based on the extraordinarily wide range of serious drug-induced "autoimmune" disorders penicillamine can produce as side effects of its use, ${ }^{371}$ it must act on some very basic immunologic mechanism $(\mathrm{s}) \cdot{ }^{370}$

Multicenter controlled trials with penicillamine in adults have demon- 
strated statistically significant improvement in such measures of arthritic activity as grip strength, hemoglobin, and ESR. The results were not all that impressive. For example, in one such study, the average ESR in the treated group was reduced from $53 \mathrm{~mm} / \mathrm{h}$ to $46 \mathrm{~mm} / \mathrm{h} .{ }^{372}$ Twenty-six percent of the patients had to discontinue the therapy because of side effects. However, some experienced clinicians were enthusiastic about penicillamine as a therapeutic agent in severe active RA inadequately responsive to conventional therapy. ${ }^{373,374}$ The drug has been used in a few hundred children with polyarthritis without systemic manifestations and is said to be about as effective as gold, equally toxic to gold, but preferred because it can be taken orally. ${ }^{375,376}$ In an uncontrolled series using a dose of $15-30 \mathrm{mg} / \mathrm{kg} / \mathrm{day}, 69 \%$ of children receiving the drug as their first slow-acting agent were said to benefit; $53 \%$ of children being treated with penicillamine after receiving gold without benefit were said to benefit from penicillamine. ${ }^{376}$ A US multicenter collaborative controlled trial failed to show benefit from $10 \mathrm{mg} \mathrm{kg} / \mathrm{day}^{377} \mathrm{but}$ children in France did a little better. ${ }^{378-380}$

Side effects of penicillamine administration may include thrombocytopenia, agranulocytosis, aplastic or hemolytic anemia; membranous nephropathy with nephrotic syndrome, ${ }^{381}$ rapidly progressive glomerulonephritis; Goodpasture's syndrome; cholestatic hepatitis; serious rashes including pemphigus; myasthenia gravis; polymyositis, ${ }^{382}$ thyroiditis; and lupus erythematosus. ${ }^{370,371}$ Vitamin $\mathrm{B}_{6}$ deficiency may be combated by simultaneous administration of $25 \mathrm{mg}$ of vitamin $\mathrm{B}_{6}$ daily at a different time than the medication. ${ }^{383}$ No harmful effects of coincident heavy-metal chelation have been noted, but they could be unrecognized, as could other effects of its lathyrogenic and collagen basement-membrane-altering properties. Serious proteinuria is more likely to occur in the same patients who develop proteinuria with gold, especially those with HLA-DR3. ${ }^{359}$ Insulin antibodies may also occur. 384

Dosage regulation of penicillamine cannot be based on serum levels, which are not available. In adults, increased side effects and few benefits have been achieved with doses in excess of $600 \mathrm{mg}$ daily. A complete blood count, platelet count, urinalysis, and chemistries are obtained prior to each increase and monthly thereafter. Urinary protein determination with filter-paper strips can be done more frequently by the parent at home. The reported incidence of side effects requiring withdrawal of medications varies from 10 to $30 \%$. Other antirheumatic medications have to be continued since a beneficial effect may not be noted for months. A favorable response is unlikely if improvement has not occurred within 6 months. ${ }^{385}$

Hydroxychloroquine. This antimalarial compound has been reported to be equally effective as gold in adults with RA but fell into disfavor when irreversible retinal toxicity was reported. ${ }^{386,387}$ Despite lack of efficacy in a large multicenter collaborative trial ${ }^{377}$ pediatric centers have continued to use it and consider it to be an effective agent with greater ease of administration 
and less toxicity than gold or penicillamine. ${ }^{388-390}$ At a dosage of 5-7 mg/kg/ day (maximum $200 \mathrm{mg}$ daily) given as a single dose, the most frequent side effect is reported to be corneal deposition, which is reversible and said to be dose-related. The most worrisome side effect is macular degeneration, which may progress after the drug is withdrawn. ${ }^{391}$ Patients with known familial tendency to macular degeneration may be at higher risk. With careful, frequent ocular monitoring and drug withdrawal, the incidence of significant retinopathy threatening vision may be kept to $1 \% .390-392$ a Glucose-6phosphate dehydrogenase (G6PD) deficiency, once thought to be a contraindication, rarely if ever causes hemolysis in patients treated with hydroxychloroquine. ${ }^{393}$ The lack of popularity of this drug represents doubts about efficacy in the face of this ocular hazard in a disorder with increased ocular risk from uveitis. If a controlled trial showed efficacy, this agent would be more widely used and has the potential for combined use with penicillamine or gold, sulfasalazine, and methotrexate. Hydroxychloroquine appears to reduce the severity of hepatotoxicity of salicylates and methotrexate. 394

When hydroxychloroquine is used with success, the drug is generally continued in full dosage for 6 months and then gradually reduced over a 2-year period. Chloroquine must be kept locked in the medicine cabinet. Death has been reported after childhood ingestion of as little as 0.8 gram. ${ }^{395}$ Myopathy, cardiomyopathy, and neuropathy may occur in addition to hematologic and dermatologic side effects. ${ }^{396,397}$

Combination of Drugs. In severely affected children it is our present practice to use combinations of drugs: (1) salicylate or other NSAID; (2) oral gold or penicillamine; (3) hydroxychloroquine; (4) sulfasalazine and, in refractory cases, all of these plus methotrexate. Our clinical impression is that this approach works better than our previous method, which was to withdraw any agent whose value did not seem proven within 6 months. At present there is no documented study which supports our approach, ${ }^{251}$ although a recent report of efficacy of methotrexate in refractory childhood arthritis may, at least in part, reflect the benefits of such polypharmacy, ${ }^{398}$ and other anecdotal reports indicate a similar approach elsewhere. ${ }^{399-401 a}$ Prompt therapy is also most essential for the group with rheumatoid-factor-positive childhood onset of adult RA. There appears to be a "window of opportunity" during which aggressive therapy may be disease modifying; scars cannot be benefitted by dangerous medications. ${ }^{402-402 a}$

\section{Corticosteroid Treatment of Severe Polyarticular JRA}

If properly used in JRA, corticosteroids may prevent blindness; enable function as opposed to the alternative of a bed-chair existence with all that implies for the child and family; and prevent death from overwhelming myopericarditis. With improper use, they may foster crippling or death. 


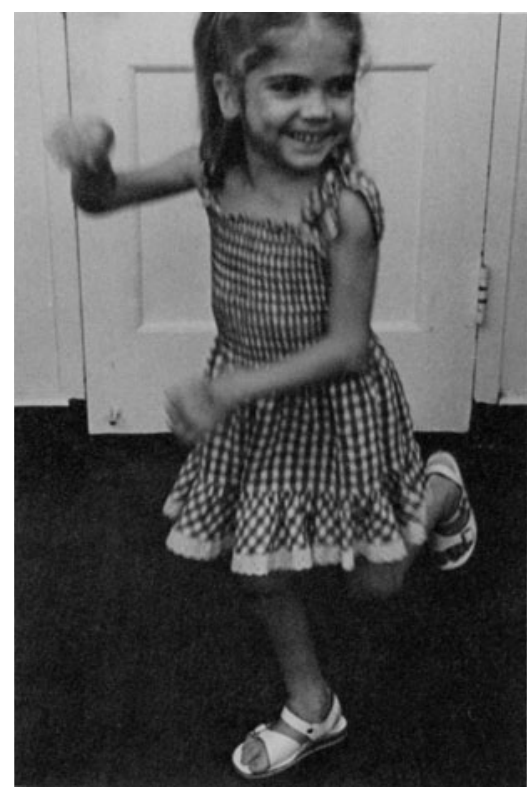

Figure 3.44. Severe systemic JRA (age 9 years, onset at age 3 years). Patient has large effusions in all joints and requires 3.9 grams of aspirin daily plus $17 \mathrm{mg}$ of prednisone on alternate mornings for control of fever and arthritis. She has had a cerebrovascular accident from which she has fully recovered and required a gastrectomy for uncontrollable hemorrhage caused by a penetrating duodenal ulcer at a time when she was receiving indomethacin, aspirin, and steroids. She has failed to be helped by hydroxychloroquine, gold, pencillamine, or plasmapheresis. The alternate-day steroids enabled her to attend school and summer camp and to lead an almost normal life despite progressive joint destruction (see Fig. 3.28). At college 10 years later she is taking methotrexate.

For some severely affected children, a small dose of prednisone on alternate mornings (together with their nonsteroidal drug and slow-acting agent) may make the difference between going to school and a reasonably normal life or becoming a bed-chair cripple (Fig. 3.44). 157,403-405 The risks of alternate-day prednisone are small when weighed against the alternatives. ${ }^{406}$ Animal studies and clinical evidence in these children indicate that loss of weight bearing is associated with worse cartilage destruction (see Figs. 3.51 and 3.52). ${ }^{407-411}$ Occasionally, we see the rare child who comes to us already wheelchair-bound, demineralized and weak, and with crush fractures and characteristic absence of cartilage at the knees and hips out of proportion to anything we ever see in our own most severely affected children (see Fig. 3.51). ${ }^{412,413}$ Much of this is secondary to entering the wheelchair. All of our patients attend regular school daily and none are ever allowed in a wheelchair. In our opinion, if prednisone is required to achieve that goal, it is well used for that purpose.

Small divided doses of prednisone can also be used in very severe polyarticular JRA ( $1 \mathrm{mg}$ four times daily). However, we have not used daily prednisone except in systemic JRA for many years and have found alternate-day regimens to be preferable. While we try to use the smallest dose possible on alternate days $(10 \mathrm{mg}$ ), we use whatever is needed for continued ambulation. Rifampin increases plasma clearance of prednisone, resulting in reduced efficacy. ${ }^{414}$ Pharmacologic maneuvers to minimize osteoporosis in steroidtreated children have not yet been adequately studied but nasal calcitonin 
may have promise in this regard ${ }^{415}$ and deflazacort may be bone sparing when compared with prednisone. ${ }^{416}$

Pulse Corticosteroid Therapy. Massive intravenous doses of corticosteroids administered over a short period of time ("pulse" therapy) have been reported to sometimes be helpful in renal graft rejection reactions, SLE, and other rheumatic diseases. ${ }^{417}$ Experience with a few arthritic children suggests that this technique may be helpful on rare occasions but that in general toofrequent injections are required. Doses of $20-30 \mathrm{mg} / \mathrm{kg}$ of methylprednisone have general been used. ${ }^{418,419}$

Intravenous Gamma Globulin. A controlled trial is underway in systemic JRA and soon to begin in polyarticular JRA. Extensive experience in Kawasaki disease has shown the agent to be relatively safe, very expensive, and difficult to administer in institutions not equipped for long outpatient IV therapy. Preliminary studies ${ }^{420}$ and our experience show that there is shortlived improvement in systemic features including fever and anemia and some improvement in arthritis. Infusions in most, but not all, patients $(2 \mathrm{~g} / \mathrm{kg})$ given every 4 weeks may reduce the need for prednisone by $80 \%$ but significantly reduce effusions and number of actively inflamed joints in only half the children. Nevertheless, gamma globulin may be a useful emergency treatment at times of drug reactions or in certain selected patients. ${ }^{421,422}$ Placenta-eluted gamma globulins are also being studied. ${ }^{423}$

Immunosuppressive Agents. The incidence of death from JRA is about $7 \%$ in reported series. ${ }^{125}$ While this is probably an overestimate, based on disproportionately severe cases being seen in large referral centers, it constitutes an underestimate for the select group in which almost all deaths occur: the severe polyarticular group characterized by persistent unremitting disease. It seems reasonable to suppose that these agents, despite their potential for early or late significant or even fatal side effects, might be appropriate for the treatment of those youngsters who are potentially threatened with severe and permanent wheelchair crippling or death, or with becoming "twisted wreckage."402,424,425

Methotrexate. Although methotrexate is an old drug not used in childhood arthritis despite its availability for more than 30 years, it is currently in wide use for refractory childhood arthritis. ${ }^{426,427}$ This reflects the poor efficacy and presumably equal toxicity 428 of gold, penicillamine, and hydroxychloroquine and azathioprine ${ }^{429}$ in a disease which, while only occasionally lifethreatening and usually remitting without meaningful sequelae with or without therapy, is awful in its worst forms. After methotrexate was documented to be relatively safe and efficacious in adults with $\mathrm{RA}^{430,431}$ it was tried in children and reported to be reasonably safe and efficacious if 
Change in overall severity score

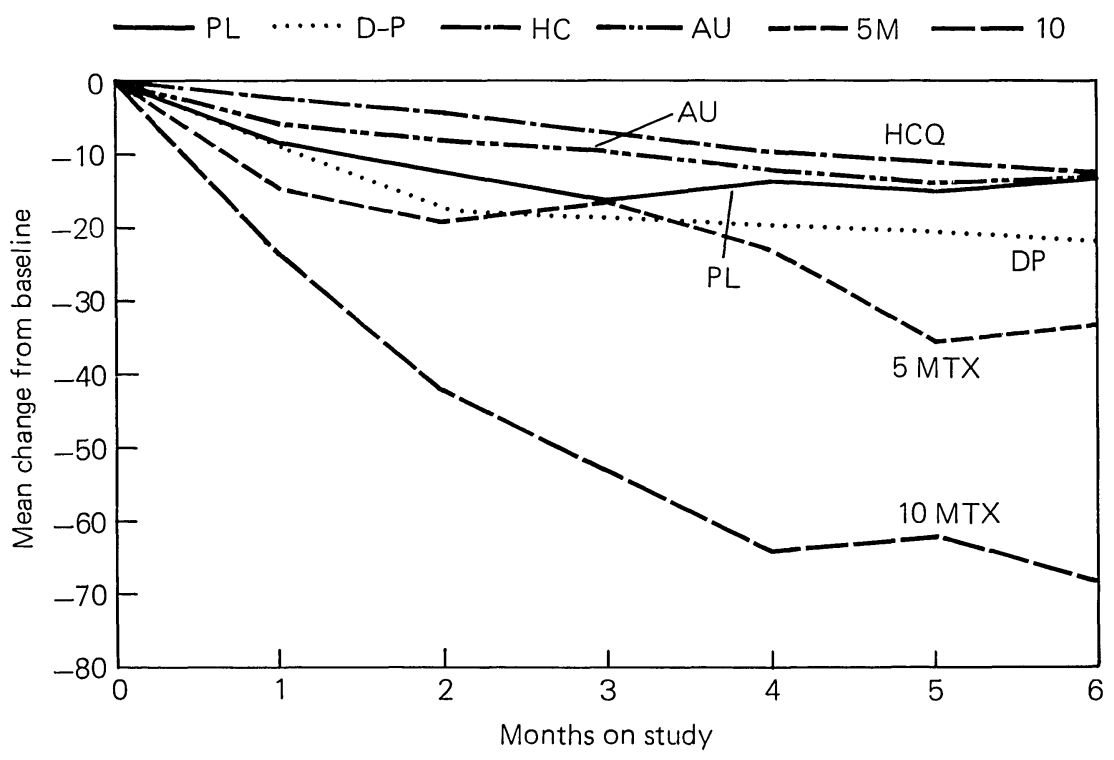

Figure 3.45. Meta-analysis of antirheumatic drug trials in juvenile rheumatoid arthritis carried out by the Pediatric Rheumatology Collaborative Drug Study Group (PRCSG). $\mathrm{PL}=$ placebo; $\mathrm{DP}=\mathrm{D}$-penicillamine; $\mathrm{HC}=$ hydroxychloroquine; $\mathrm{AU}=$ auranofin; $5 \mathrm{M}=$ methotrexate $\left(5 \mathrm{mg} / \mathrm{mm}^{2} /\right.$ week); $10=$ methotrexate $\left(10 \mathrm{mg} / \mathrm{mm}^{2} /\right.$ week $)$. Only $10 \mathrm{mg} / \mathrm{mm}^{2} /$ week methotrexate was distinguishable from placebo with respect to efficacy. (Kindly supplied by Dr. E.H. Giannini, PRCSG.)

given in a dose of $10 \mathrm{mg} / \mathrm{mm}^{2} /$ week. ${ }^{432-434}$ Some authors measure serum levels and adjust doses upward for those who have poor absorption of drug; for these studies the medication is given with water after an 8-h fast and the fasting blood level is determined $1 \mathrm{~h}$ later. ${ }^{426}$ Levels less than $0.6 \mu \mathrm{g}$ tended to be associated with less than optimal results and higher levels, achieved with doses as high as $1.1 \mathrm{mg} / \mathrm{kg} /$ week, were sometimes effective and tolerated when lower doses had failed. ${ }^{436,437}$ Salicylates, NSAIDs, and sulfonarnides may increase levels and, therefore, both efficacy and toxicity. ${ }^{438,439}$ Growth hormone may hasten excretion of all medications.

While methotrexate may slow radiologic progression of disease, it does not prevent it. $440-441$ a Severe flare-ups in disease may be expected following withdrawal. ${ }^{442,443}$ In adults, cumulative toxicity over 5 years amounts to $54 \% .{ }^{444}$ Most liver injury is mild and clinically insignificant ${ }^{445-449 a}$ but occasionally severe injury occurs (cirrhosis). ${ }^{450-452 a}$ Five percent of adults have severe pulmonary toxicity. ${ }^{453}$ Stomatitis, leukocytoclastic vasculitis, central-nervous-system dysfunction, xylose malabsorption with jejeunal his- 
tologic changes, and bone-marrow suppression may occur. ${ }^{453-456}$ Osteopathy, delayed fracture healing, and increased risk of postsurgical complications are important considerations in children requiring scoliosis or other surgery. ${ }^{457,458}$

Methotrexate improves quality of life for some patients $(29 \%)^{459}$ and is a useful agent, better than and perhaps better tolerated than gold or penicillamine $^{460}$ (Fig. 3.45). However, it is not the ultimate agent: anemia was improved in only $43 \%$ of children, number of swollen joints reduced by only $46 \%$, swelling in joints with persistent swelling reduced by only $52 \%$, and corticosteroid dosage reduced in only one-third children who required steroids. ${ }^{427}$ Results are better if it is introduced early in the course of disease and may be enhanced by sulfasalazine and hydroxychloroquine, ${ }^{436,437}$ and possibly by increased or parenteral dosage with leucovorin rescue. ${ }^{461}$ Intramuscular injections may be given by patients or parents; ${ }^{360}$ subcutaneous injections may be bioequivalent to intramuscular. ${ }^{437}$

Azathioprine. In adults, no difference could be shown between azathioprine $150 \mathrm{mg} /$ day and methotrexate, $15 \mathrm{mg}$, once weekly, at 24 weeks; it is generally thought that methotrexate works faster but this could not be documented in a controlled trial. ${ }^{462}$ More than $10 \%$ of children treated with $2.5 \mathrm{mg} / \mathrm{kg} /$ day in a controlled trial had to be withdrawn due to toxicity, primarily neutropenia/infections, and benefit over placebo was minimal but statistically significant at 16 weeks for subjective total assessment and functional capacity. ${ }^{463}$ Despite increased risk of malignancy with long-term usage, ${ }^{464}$ and hepatotoxicity and hypersensitivity reactions requiring discontinuation within 1 year in about $12 \%$ of patients, ${ }^{465}$ azathioprine has a reasonable safety profile ${ }^{466}$ if only it were more effective. ${ }^{465 a}$

Cyclophosphamide. Cyclophosphamide at $75 \mathrm{mg} /$ day inhibits erosions and relieves symptoms in adult RA but has generally unacceptable risks; ${ }^{467,468}$ in life-threatening disease the risks may be acceptable. ${ }^{469-472}$ Intravenous pulses $\left(500 \mathrm{mg} / \mathrm{mm}^{2}\right)$ at 4-6-week intervals provided little improvement and all patients withdrew after 6 months; ${ }^{473}$ higher doses, similar to those used in SLE, have not been studied. Combinations of cyclophosphamide, azathioprine, and hydroxychloroquine have been tried in adults. ${ }^{474}$

Chlorambucil. Chlorambucil, $0.01-0.16 \mathrm{mg} / \mathrm{kg} / \mathrm{day}$, given to children with JRA-associated amyloidosis, intractible vision-threatening uveitis, or uncontrolled systemic disease, was moderately effective ${ }^{123,124}$ but associated with $100 \%$ chromosomal injuries, ${ }^{475}$ occasional leukemia, ${ }^{476}$ and gonadal injury. ${ }^{477}$ Melphalan and colchicine might also be worthy of trial in JRA with amyloidosis. ${ }^{478,479}$

Cyclosporine. Adults and one child with arthritis unresponsive to conventional therapy have been treated with cyclosporine. ESR and rheumatoid fac- 
tor have not been reduced but inflammation and functional symptoms have improved. ${ }^{480-487}$ Nephropathy limits dosage to $4 \mathrm{mg} / \mathrm{kg}$, probably the maximum safe dose; close safety monitoring is essential, especially if higher doses are required. ${ }^{488-493}$ Children generally require higher doses than adults. ${ }^{492}$ In a recent study in adults, cyclosporine was no better than penicillamine, ${ }^{493}$ and increases risk of lymphoma. ${ }^{493 a}$

Levamisole. This potent antihelminthic has some anti-inflammatory effects at high dosage and has thymominetic effects on T lymphocytes. ${ }^{494}$ The beneficial effects in adults with RA are said to be similar to those obtained with gold and penicillamine, but not as good as those found with azathioprine. ${ }^{495}$ A high incidence of fatal agranulocytosis, seizures, and coma has prevented adequate study in children and suggests that levamisole is unlikely to be useful in JRA. ${ }^{496,497}$ However, unusually high doses were given to some of the children. New agents derived from levamisole or with similar pharmacologic properties and less toxicity may be discovered and be of more use in childhood arthritis. ${ }^{497}$

Plasma Exchange and Lymphocyte Depletion. Reducing the amounts of circulating antibody and immune complexes in antibody- or immunecomplex-mediated diseases by plasma exchange is a technique used in Goodpasture's syndrome, SLE, and myasthenia gravis. Initial reports, based on somewhat sketchy evidence, have been enthusiastic. Plasmapheresis, lymphoplasmapheresis, and lymphapheresis have been tried in RA with varying success. ${ }^{498-500}$ In one report of plasma exchange in JRA, success was dependent on the simultaneous administration of fresh-frozen plasma and was associated with an accidental death. ${ }^{501}$ Apheresis at present must be considered a research procedure rather than an accepted mode of therapy.

Since high titers of rheumatoid factor or significant immune complexes have usually not been demonstrated in JRA, the mode of action of plasma exchange, if it worked, would be entirely unknown. We have tried plasmapheresis in one terribly ill systemic JRA patient. A femoral vein catheter was required. Blood transfusions were also required since the child was too small to provide blood for "priming" of the machine. Prompt improvement was seen in terms of arthritis, serum levels of immune globulins, and ESR. However, within a few weeks, the patient had resumed her prior state. The technical difficulties and repetitive hazards of doing this procedure in this small child outweighed its small benefits.

The use of total body lymphoid irradiation to achieve lymphocyte depletion in adult RA has not provided long-term improvement sufficient to warrant trials in children. ${ }^{400}$ Thymopoietin, ${ }^{502}$ typrilose, ${ }^{503}$ gamma interferon, ${ }^{504}$ tetracycline, ${ }^{505}$ and fish oil ${ }^{506}$ are safer agents being studied in adults. Arthritic children who developed myasthenia gravis and were subjected to thymectomy have had complete remission of their arthritis. ${ }^{507} \mathrm{~A}$ few, but only a few, patients improve when they take birth-control pills ${ }^{508}$ or 
amantadine. ${ }^{509}$ Extracorporeal photochemotherapy is now being studied in adults. ${ }^{510}$ Parents try many unconventional remedies. ${ }^{511}$

\section{Pauciarticular Juvenile Rheumatoid Arthritis}

It has long been recognized that arthritis in childhood frequently affects only one or a few joints. ${ }^{3,12}$ In 1977, the ARA-JRA criteria committee agreed to classify children who, at 6 months after onset of arthritis, have disease limited to one to four joints as a pauciarticular subset. ${ }^{13}$ This is the most frequent form of arthritis in childhood and accounts for an increasing proportion of patients seen in large childhood arthritis clinics (Table 3.4). Large joints, especially those of the lower extremities, are most frequently affected, and involvement is often asymmetrical and spotty.

The pauciarticular group has a better prognosis than either the systemic or polyarticular groups. Most patients will make a full recovery from the arthritis and have no disability. However, $11-37 \%$ of pauciarticular onset children will ultimately have a polyarticular course, and some significant disability in individual joints may occur. Visual impairment from chronic uveitis is also a significant risk in this group. ${ }^{82}$

Just as it has become apparent that it is difficult to make general statements about children with JRA without dividing them into clinical subsets, it has become obvious that what has been called pauciarticular JRA is an amalgam of different disorders. However, no two authorities agree at this moment on how to subdivide this group. Everyone recognizes the need to identify those patients who really have the disorder AS. ${ }^{22,275,512}$ In our current schema of subdividing JRA, we have used histocompatibility testing as an aid to subset characterization. For purposes of study and prognosis as well as a useful guide to drug management, we find it most helpful to identify all HLA-B27 patients and consider them a separate disorder. Similar patients have been reported elsewhere as one subset of pauciarticular JRA. ${ }^{82}$ HLAB27-associated arthritis is discussed in Chapter 4.

All authors agree on a second clinical subset of pauciarticular disease, which is often characterized as "little girls with one swollen knee, a positive test for antinuclear antibody, and a high risk of chronic uveitis." 18,82 In one study, $10 \%$ of arthritic children belonged to this subset. (Only $65 \%$ of these were ANA-positive. ${ }^{82}$ Some authors have included all patients with chronic uveitis as a single subset (i.e., not limited to females). Children with the onset of pauciarticular arthritis prior to age 5 tend to be in this subset. Recent studies in our laboratories and others have shown that HLA-B27-negative, early onset pauciarticular JRA with uveitis is associated with HLADR5. 10,18,513 Other HLA-D-region antigens are associated with increased risk of pauciarticular JRA in some populations; a single mechanism (shared epitope) may be responsible for the increased susceptibility. ${ }^{514}$

The characterization of pauciarticular JRA into subsets is not just of clin- 
ical interest. With recent evidence suggesting that JRA may represent a series of disorders with T-cell defects, it is reasonable to presume that different patterns of disease may be a reflection of different variations in immunologic function. ${ }^{515}$ For purposes of study, we are now dividing the HLA-B27negative pauciarticular patients into two subsets: DR5-positive and DR5negative.

\section{HLA-DR5-Associated Pauciarticular JRA}

Among 39 pauciarticular-onset patients known to be HLA-B27-negative, we found 23 DR5-positive patients. ${ }^{10,513}$ The tested population was not unselected and was weighted for uveitis, so it may not be a totally accurate representation of the constituency of the total B27-negative pauciarticular group. Seven DR5 patients were males; one of these had uveitis. Thus, while males (seven of 23) are not as frequently affected as females (16 of 23), they did not constitute an insignificant part of this population. The number of affected joints at onset averaged only 1.3. In 17 of 23 patients (74\%), the onset was monarticular. The knee was most frequently affected (12 of 23), with hip and ankle the other relatively frequently affected joints (five of 23). Elbow, finger, foot, and TMJ were all affected in single instances; in no cases were the wrists or shoulders affected at the time of onset of arthritis in this population. The arthritis tended to be very mild, to respond to aspirin, and to remit relatively quickly. Recurrent attacks were common, however, and two patients developed a polyarticular course.

The age at onset of this arthritis differs in association with the presence or absence of uveitis. The average age at onset in girls with uveitis was 3.8 years (in the single boy, 3 years), while in girls without uveitis, it was 8.2 years, and in boys without uveitis, 7.5 years. The pattern of arthritis did not vary with age.

This association of DR5 with pauciarticular JRA and especially with girls with JRA and uveitis has since been confirmed by Glass et al. ${ }^{18}$ After discussion with us, they selected 45 pauciarticular JRA patients, heavily weighted for females (41) and uveitis (24). Of the 45, 28 were HLA-DR5; 17 of 24 with uveitis were HLA-DR5. As expected from prior reports, ANA was found especially frequently in the DR5-positive girls with uveitis. The relationship between DR5 and this subset of childhood arthritis has been found in all populations so far examined. Our preliminary data suggest that other DR associations found only in individual geographic areas may represent population-biased samples. However, further study is required.

\section{Non-B27, non-DR5 Pauciarticular JRA}

Of the 39 HLA-B27-negative patients tested, 16 were also DR5-negative; of these, six were boys and 10 were girls. The average age at onset of arthritis 
was 6.4 years for boys and 5.9 for girls. There were no apparent differences to distinguish the pattern of arthritis in this subset from the total pauciarticular group, but individual patients might most resemble one of the other subsets, that is, HLA-B27-associated disease, DR5-associated disease, or polyarticular seropositive RA associated with DR4.

\section{Prognosis}

The overall prognosis for the entire pauciarticular JRA group is good, with relatively little scarring except in the eyes (see below). However, more severe involvement requiring joint replacement occurs in the B27 group (see Chapter 4), and recent studies indicate that the prognosis for the knees of the DR5 uveitis group may not be as good as we had previously thought. ${ }^{516}$ While in our short-term follow-up period the prognosis for joint function seemed good, in a larger long-term series of patients with chronic iritis reported by Kanski, 20\% of patients had "severe disease" and ultimately required surgical joint replacement. ${ }^{517}$ Pauciarticular patients carrying HLA-DR13-DW18 and DQ6-DW18 are more likely to have persistent disease. ${ }^{518}$

\section{Treatment}

In most pauciarticular patients, aspirin, $90 \mathrm{mg} / \mathrm{kg} /$ day (max. $3.6 \mathrm{~g}$ daily) in four divided doses, provides excellent control of the arthritis. A few children respond better to tolmetin or naproxen or tolerate it better, and these agents are now the first choice of most parents. Teenagers may prefer other nonsteroidal anti-inflammatory agents. In our clinic gold, penicillamine, and hydroxychloroquine are not used in the treatment of pauciarticular disease since the toxic hazards of these agents would seem to outweigh their potentia therapeutic promise. We use sulfasalazine extensively in pauciarticular patients.

Antirheumatic medication is continued until the patient has been asymptomatic and has normal laboratory studies for 6 months. Remission is defined by being asymptomatic and having normal laboratory studies for a period of 6 months after cessation of all treatment.

Intra-articular Corticosteroid Injections. Children do not like injections into their joints; general anesthesia may be required for children under age $6 .{ }^{519}$ The beneficial effect, if there is any, tends to wear off in days. Intrasynovial injections are occasionally complicated by the introduction of bacteria into the arthritic joint, creating a very difficult diagnostic and therapeutic problem. Experimental evidence suggests hydrocortisone may have a deleterious effect on damaged cartilage in young animals. Repeated injections may foster total joint destruction. Leakage of steroid around the needle tract may cause 
permanent unattractive atrophy of skin and subcutaneous tissues. ${ }^{520}$ Fifty percent of children treated with intra-articular steroids develop calcifications in and around the treated joint, most of which are asymptomatic, but 3/55 develop avascular necrosis, a very serious complication..$^{21,522}$ Nevertheless, there are occasions when one injection of long-acting steroid into a single large knee effusion, after removal of fluid, provides great relief of pain, allowing mobilization of the stiff joint and correction of deformity. ${ }^{13,523,524}$ The beneficial effect, in some rare instances, may be extremely long-lasting. The risk of a single injection is small, but we do not give repeated injections. Steroids must not be injected at the time of arthroscopy as the risk of infection is high. ${ }^{525}$

\section{Iridocyclitis (Anterior Uveitis)}

The association of iritis with arthritis in children; the distinction of this subacute or chronic iritis, which was persistent from the form of iritis that was acute and paroxysmal; and the observation that the parents of children with iritis usually had arthritis were all reported by Jonathan Hutchinson in Lancet in $1873,{ }^{526}$ a quarter of a century prior to Still's description of JRA and over a century before we again recognized the heritable tendency.

Subacute or chronic inflammation of the iris and ciliary body occurs in $20 \%$ of all children with JRA (Fig. 3.46). Both eyes are ultimately affected in $70 \%$ of children with uveitis. Patients tend to have recurrent attacks; in $21 \%$ of cases, the duration of eye disease exceeds 10 years. ${ }^{517}$ Two percent of arthritic children, or $6 \%$ of all with pauciarticular onset seen prior to 1960 , were totally blind in 10 years. ${ }^{42,527,528}$ Arthritis precedes the onset of uveitis in almost all $(92 \%)$ cases. ${ }^{517,529}$

\section{The Susceptible Population}

If the B27 subset is excluded, about $90 \%$ of arthritic children with uveitis are girls. In some series, $60-90 \%$ of them have antinuclear antibodies. ${ }^{529,530}$ Although the disease is almost always pauciarticular (often monarticular) at onset, it need not remain pauciarticular, and some severely incapacitated patients have uveitis. The mean age at onset of arthritis in the uveitis population is 3.7 years. ${ }^{517}$ The knee is the single most commonly affected joint. The arthritic population characterized as little girls with swollen knees and a positive ANA test has a greater than $40 \%$ risk of uveitis. In our studies, $90 \%$ of arthritic children with subacute or chronic uveitis were of a single HLADR type-DR5. ${ }^{513}$ Certain DR5 haplotypes predispose more than others. ${ }^{531}$ Patients with pauciarticular-onset arthritis remain at risk for uveitis for many years. In $21 \%$ of cases, uveitis began more than 5 years after the onset of arthritis. ${ }^{517}$ 


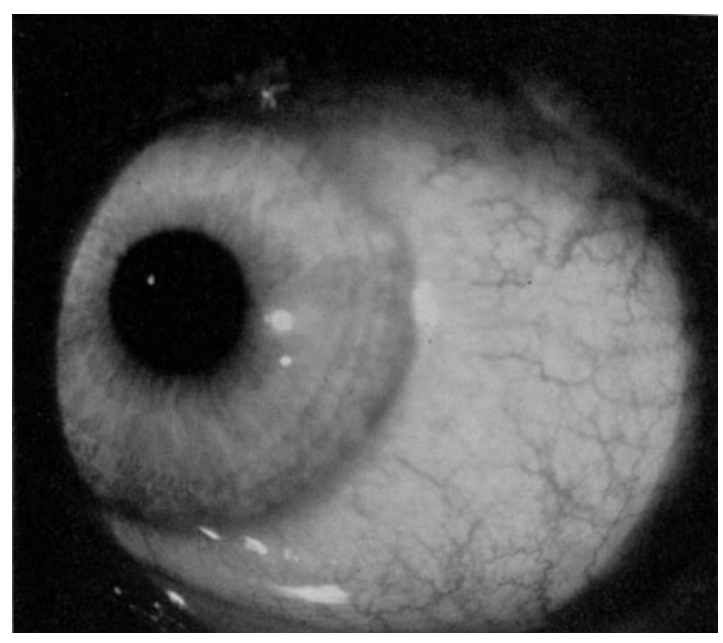

Figure 3.46. Although the subacute or chronic uveitis associated primarily with pauciarticular JRA (especially common in little girls with antinuclear antibodies) is generally found only by routine screening slit-lamp examinations, occasionally patients will have a "ciliary-flush" indicating inflammation.

\section{Diagnosis}

Most patients are asymptomatic early in the course of their disease, although mild redness of the eye or visual complaints occur occasionally (Fig. 3.46). Thus, early diagnosis depends on routine slit-lamp screening of all arthritic children. This is carried out at 3-month intervals in the most susceptible population. The ophthalmologist notes the number of cells in the anterior chamber and the amount of protein precipitate (flare), using a standard grading system. Fresh keratic precipitates are noted if present. Unfortunately, in published reports, up to $18 \%$ of patients already had band keratopathy, and $9 \%$ had cataracts at the time of initial diagnosis. ${ }^{517,532}$

\section{Course}

If the disease is unilateral when first seen and the second eye becomes involved, it generally is affected within a year. Although attacks of uveitis may be brief, they tend to recur. ${ }^{517}$

All patients are not equally severely affected, and it seems unlikely that all have the same potential for scarring and blindness. ${ }^{530}$ Our routine screening procedure identifies some patients with inconsequential disease. However, in the largest published experience (160 cases), Kanski reported some loss of vision in $43 \%$ of affected eyes. ${ }^{517}$ Twenty-six percent of affected eyes in his series lost all vision or retained only light perception or the ability to count fingers; $17 \%$ of affected children were totally blind. It is hoped that with earlier diagnosis and treatment and modern surgical techniques, these figures are a reflection of the past rather than current experience. ${ }^{528}$ 
ACUTE

Prednisone ( $2 \mathrm{mg} / \mathrm{kg} /$ day; max. $80 \mathrm{mg}$ ) plus local treatment*

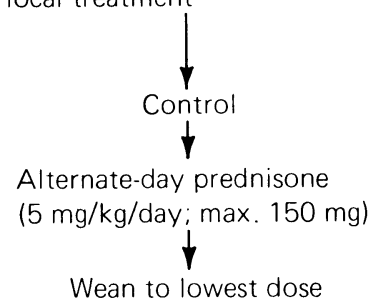

*Acute uveitis following ocular surgery may require q $3 \mathrm{~h}$ doses for control during the initial postoperative week and in adults may require higher total doses.
SUBACUTE

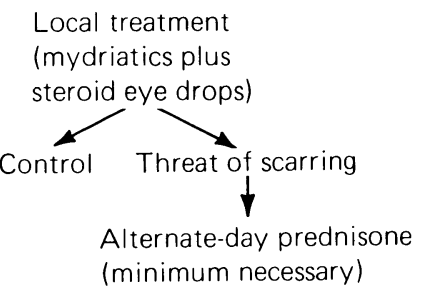

RESISTANT CASES

Methotrexate

(10 $\mathrm{mg} / \mathrm{kg} /$ week)

Figure 3.47. Treatment module for uveitis.

\section{Medical Treatment}

Most of our patients are already receiving NSAIDs when they develop uveitis. ${ }^{533}$ It is not yet known whether sulfasalazine decreases the frequency of iritis. ${ }^{533 a}$ Uveitis is treated with mydriatics and topical corticosteroid drops administered at frequent intervals. ${ }^{528,530,534}$ If a prompt response is not obtained, systemic steroids are instituted (Fig. 3.47). We have not found subtenon injections of steroid a practical approach to therapy and have cared for one child who was blinded as a result of an inadvertent arterial injection. ${ }^{535}$ We use prednisone, $1-2 \mathrm{mg} / \mathrm{kg} / \mathrm{day}$ in divided doses, until the inflammation is completely controlled (usually a few weeks) and then change to an alternate-day regimen $(2-5 \mathrm{mg} / \mathrm{kg} / \mathrm{dose})$. If the inflammation is controlled, the dose is gradually reduced with careful monitoring of the eyes. The regimen is aimed at preventing visual scars and accepts steroid side effects in an effort to prevent blindness.

This program, together with frequent monitoring of asymptomatic arthritic children, has the potential to prevent blindness totally from latediagnosed or inadequately controlled uveitis. ${ }^{528}$ Nevertheless, we continue to see patients who are blinded by complicated cataracts secondary to inadequately treated uveitis. ${ }^{536,537}$

While in our clinic with this regimen we have had no loss of visual acuity in $86 \%$ of children with uveitis in whom the diagnosis was made prior to loss of visual acuity, ${ }^{529}$ others have not had similar success and report limited steroid responsiveness. ${ }^{517}$ There has been too little experience with immunosuppressive drugs in steroid-resistance uveitis to allow meaningful comment ${ }^{538-540}$ but new studies with methotrexate are promising. ${ }^{540 a}$ 
Table 3.18. Results of Cataract Surgery in JRA

\begin{tabular}{lcccccc}
\hline Visual Acuity & $\begin{array}{c}\text { Smiley and } \\
\text { Kanski }\end{array}$ & $\begin{array}{c}\text { Chylack } \\
\text { et al. }{ }^{534}\end{array}$ & $\begin{array}{c}\text { Key and } \\
\text { Kimura }\end{array}$ & $\begin{array}{c}\text { Praeger } \\
\text { et al. }{ }^{543}\end{array}$ & $\begin{array}{c}\text { Kanski } \\
\text { and } \\
\text { Crick }^{*}\end{array}$ & $\begin{array}{c}\text { Diamond } \\
\text { and } \\
\text { Kaplan }\end{array}$ \\
\hline 20,545 \\
20/40-20/120 & 8 & 6 & 11 & $4^{+}$ & 14 & 5 \\
20/30 or better & 3 & 2 & 9 & 3 & 5 & 2 \\
$\quad$ Totals & 1 & 0 & 3 & 13 & 15 & 8 \\
\hline
\end{tabular}

*Acuity groupings slightly different: Kanski and Crick: 20/120 or less, 20/60-20/80, 20/40 or better; Diamond and Kaplan 20/100 or less, 20/70, 20/25, or better.

† One failure related to amblyopia exanopsia and three to uncontrolled glaucoma.

\section{Surgery for Band Keratopathy}

When vision is obstructed by calcium deposits in the cornea, removal of the band keratopathy by chelation and curretage is a simple and generally successful procedure. ${ }^{541}$ Laser surgery may help in resistant cases.

\section{Cataract Surgery in Patients with Uveitis}

Until recently, the surgical removal of a complicated cataract in arthritic

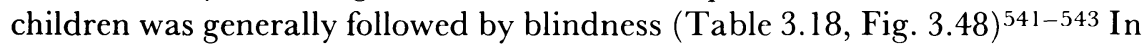
1976, we reported a two-phase new approach to these cataracts; removal with the new surgical technique of phacoemulsification and intraoperative and postoperative medical support with daily high-dose steroid therapy. ${ }^{543}$ In Dr. Praeger's hands, this technique has now been entirely satisfactory in 13 of 17 cases (Table 3.18, Fig. 3.49). Improved results with new techniques for lensectomy rather than phacoemulsification, but without systemic steroids, have been reported by Kanski and Flynn. ${ }^{541,542,544}$ The published results do not equal the combination of phacoemulsification and aggressive steroid therapy. Kaplan and Diamond, after demonstrating that postvitrectomy uveitis in the rabbit was prevented by vigorous steroid therapy, used a steroid regimen similar to ours but with a different surgical procedure (lensectomy with vitrectomy) for patients with complicated cataracts. ${ }^{545}$ Their results also do not equal those with phacoemulsification, but their patient population did not include children and may not have been analogous to ours. We have also used our steroid regimen with lensectomy and vitrectomy without phacoemulsification in two children. Both operations were successful in restoring vision, but more steroids seemed to be required than have been required in those cases done with phacoemulsification. Chylack has also reported phacoemulsification to be the procedure of choice in these children. ${ }^{534}$ Thus, pending further study, our recommendation would be administration of steroids to completely control inflammation on the day prior to and during 

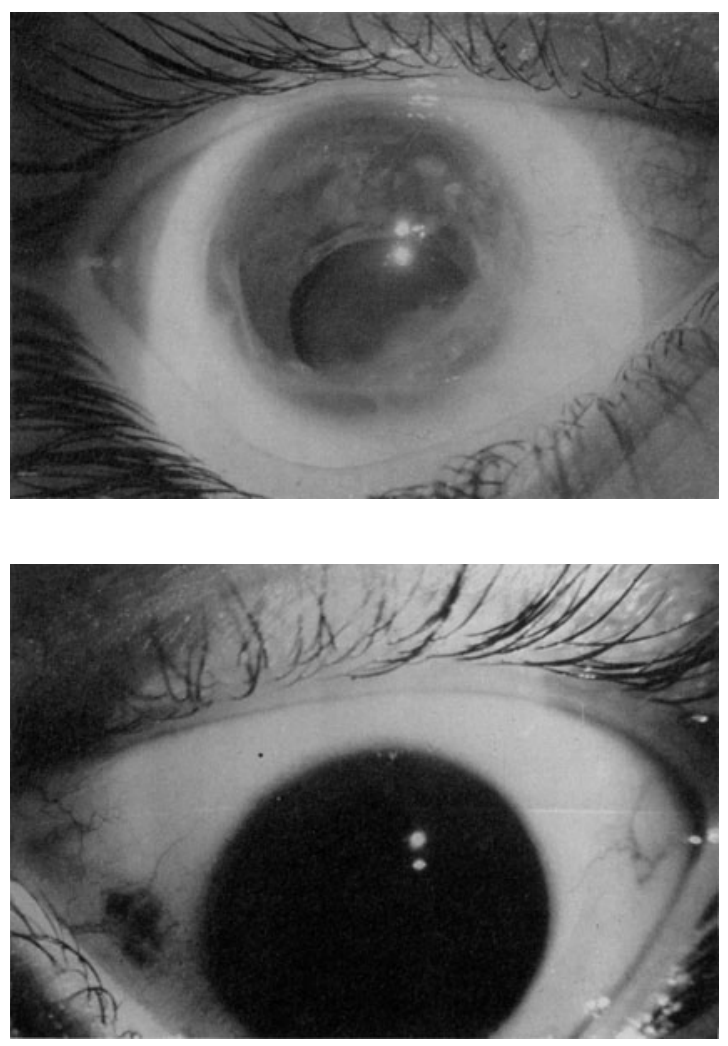

Figure 3.48. Appearance of eye following surgery with old techniques aimed at removing the complicated cataract that results from uveitis. Very few patients we operated on retained useful vision (Table 3.18).

Figure 3.49. Note clear appearance of the eye a few months after surgery using phacoemulsification and highdose perioperative steroids. Photo shows superior sphincterectomy and inferior iris sphincterotomy with pericentral opacification of intact posterior capsule. Note iris synechia to lens capsule. (Courtesy of Dr. D. Praeger.)

surgery and in sufficient dosage to control inflammation postoperatively with cataract removal by phacoemulsification. The surgical technique and steroid regimen are detailed elsewhere. ${ }^{543}$ Unilaterally, blind eyes in young children must be operated on promptly to avoid amblyopia exanopsia. Glaucoma must be adequately treated when present or a blind eye will result despite successful cataract surgery. Trabeculodialysis is said to work best. ${ }^{541,542}$

\section{Arthritis with Psoriasis}

All forms of arthritis may occur coincident with psoriasis, but arthritis that mainly involves the distal interphalangeal joints of the hands is most distinctive and is usually called "psoriatic" (Fig. 3.50). Less than half of all arthritic children with psoriasis have this form of arthritis. ${ }^{546-549}$ Family history of psoriasis, and nail pitting or onycholysis suggest this subset; dactylitis may be the presenting manifestation. Arthritis with these manifestations in childhood is usually pauciarticular in onset but often progresses to a polyarticular 


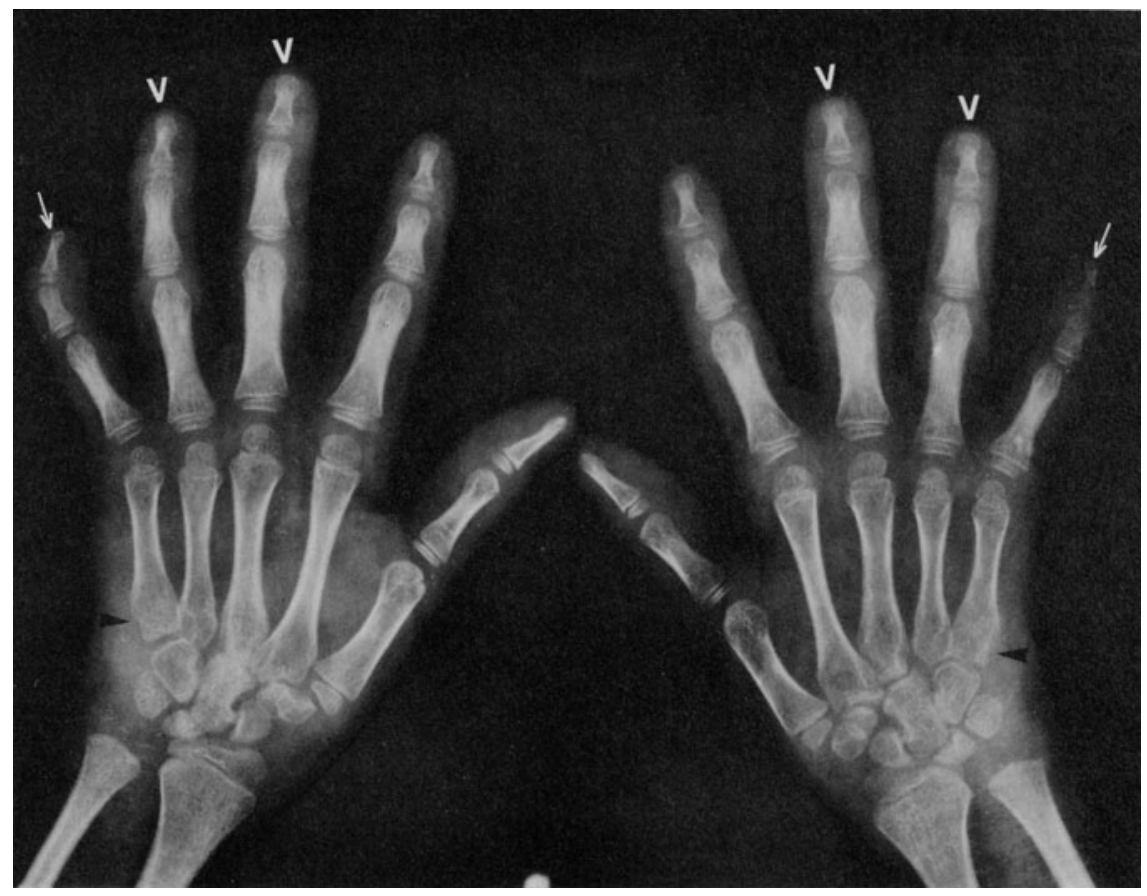

Figure 3.50. Hands of a 9-year-old boy with congenital familiar psoriatic erythroderma, showing loss of soft tissues in the distal finger tufts (arrowheads), with beginning loss of bone substance and DIP-joint arthritis in the fifth fingers (arrows). There are extensive arthritic changes at the wrists with crenation of the carpal bones and loss of joint space. Note severe erosions visible at the lateral bases of the fifth metacarpal bones (black arrowheads). The findings are typical of those seen in other forms of psoriasis.

course ${ }^{549}$ Sacroiliitis occurs in $30 \%$ of children with psoriasis and arthritis and, when present, is generally associated with the presence of HLA-B27. Iritis occurs in about $8 \%$.

Females are more commonly affected with arthritis/psoriasis (2.6:1). The mean age of onset is high for childhood arthritis (9-10 years of age). Arthritis usually precedes the appearance of the psoriatic skin lesions. Some authors suggest that synovial-lining cells are less affected, and there is increased inflammation, fibrosis, and thickening of the walls of small and medium-sized synovial arteries when compared with other types of arthritis. ${ }^{550}$

Although psoriatic arthritis in childhood is generally a mild disease, it tends to continue into adult life and, in some cases, is associated with rapid joint destruction. Management is similar to other forms of childhood arthritis and depends on the extent of the joint involvement. Colchicine, ${ }^{551}$ cyclosporine, ${ }^{552}$ and 1,25-hydroxyvitamin $\mathrm{D}_{3}{ }^{553}$ may be effective. We have used 
cyclosporine where all else has failed, with great success; $5 \mathrm{mg} / \mathrm{kg} /$ day in two divided doses may be the best starting dose, with close attention to the potential for blood-level alterations caused by drug interactions. ${ }^{554}$ Unfortunately, patients' illness may be exacerbated as soon as one tries to withdraw the medication, and the risks increase with long-term use. ${ }^{554-556}$

\section{Exercises as Therapy in Childhood Arthritis}

Movement and weight bearing are important in preventing accelerated destruction of cartilage. Evidence from animal studies shows that experimentally damaged cartilage suffers worse destruction if prohibited from weight bearing and exercise. ${ }^{407,557}$ Radiographic regrowth of hip cartilage/fibrous tissue allowing improved function has similarly been demonstrated following ambulation of bedridden children with hip destruction from JRA (Fig. $3.51) .{ }^{411}$ It is also generally not understood that joint capsular structures and muscle undergo adaptive shortening during immobilization even in the absence of inflammation. ${ }^{410,558}$ When combined with inflammation in the synovium and all the periarticular structures, immobilization results in the most aggressive functional impairment of hips and knees, resulting in a bedridden condition (Fig. 3.52). Once bedridden, cartilage destruction and contractures about the joints accelerate.

The reason for the ghastly radiographic appearance of the hips and knees of bedridden JRA patients is not just JRA but the vicious cycle of JRA combined with immobility and lack of weight bearing (Fig. 3.51). ${ }^{411}$ Sometimes this cycle is begun by well-meaning physicians who do not understand these physiological mechanisms. The natural tendency is to avoid use of painful joints. It is often hard to convince physicians of the crucial importance of walking on damaged legs in JRA. Convincing parents and children in pain requires a thorough understanding of the critical necessity for ambulation and of the horrendous physical crippling created by immobility in chronic polyarticular JRA. ${ }^{411}$

Complete immobilization of the arthritic joint (as in circular plaster) is never indicated and may result in accelerated loss of muscle strength and prolonged or permanent loss of function. We continue to see children who are put in plaster by orthopedists even though x-rays are normal "just in case" they mave missed a fracture. This practice is to be discouraged.

The primary goal of the physical and occupational therapist in JRA is the maintenance of function of individual joints, of the child, and of the family. Physical measures to help relieve pain and deformity and to preserve and restore motion and strength are the most-often-emphasized tools of the physicians and allied health personnel in the department of physical medicine and rehabilitation. Their role in the care of these children is greater than the sum of the parts. They exude confidence and show their commitment to function; they emphasize the positive-what the child can do-and fight to minimize 


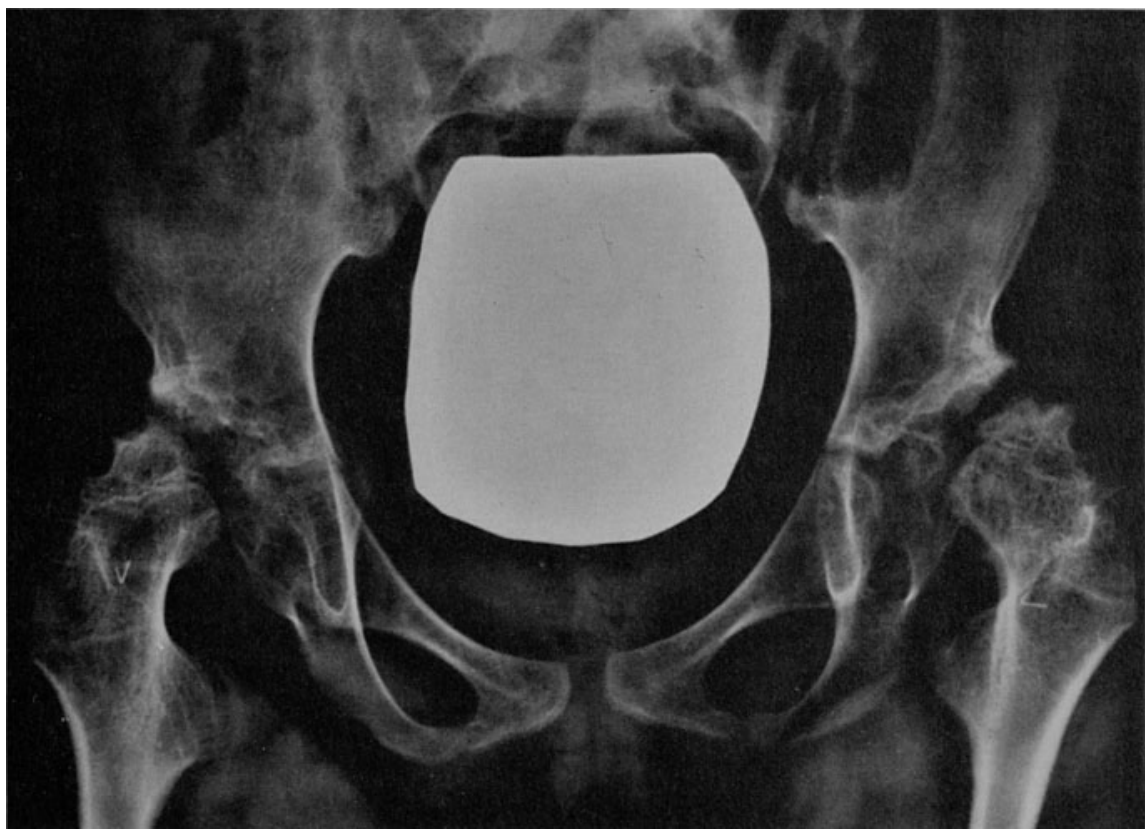

A

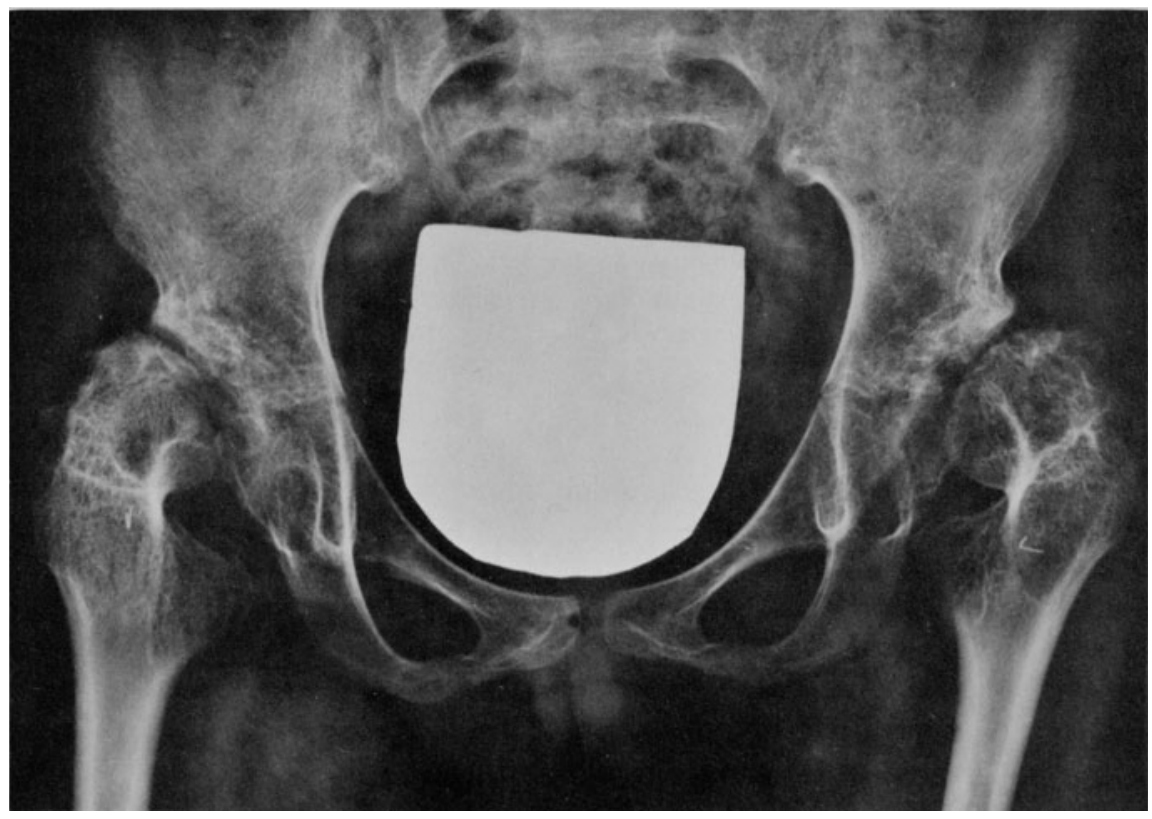

B

Figure 3.51. Hip radiographs of a 9-year-old Korean girl (Fig. 3 52) brought here for treatment of systemic JRA. The child had been bedridden for 5 years. (A) Remineralization and filling-in of bony defects 2 years after patient began to fully bear weight on the joints. ${ }^{560}$ (B) Metallic objects are broken acupuncture needles. (Reprinted with permission from Jacobs et al., ref. 413.) 


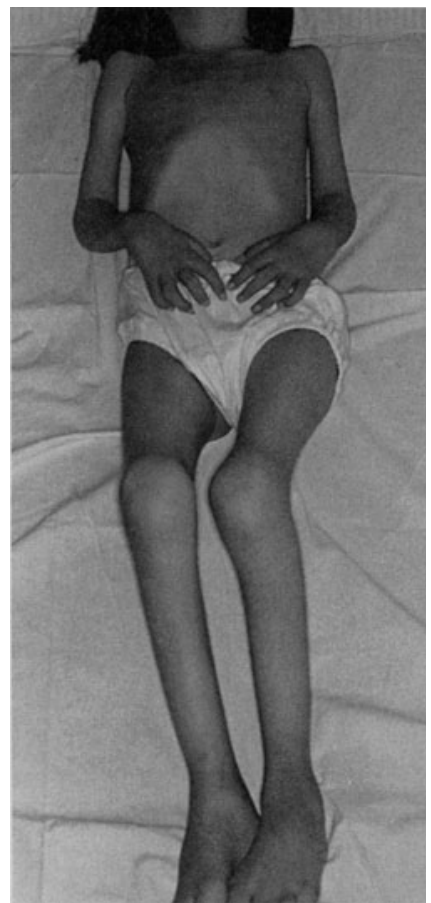

A

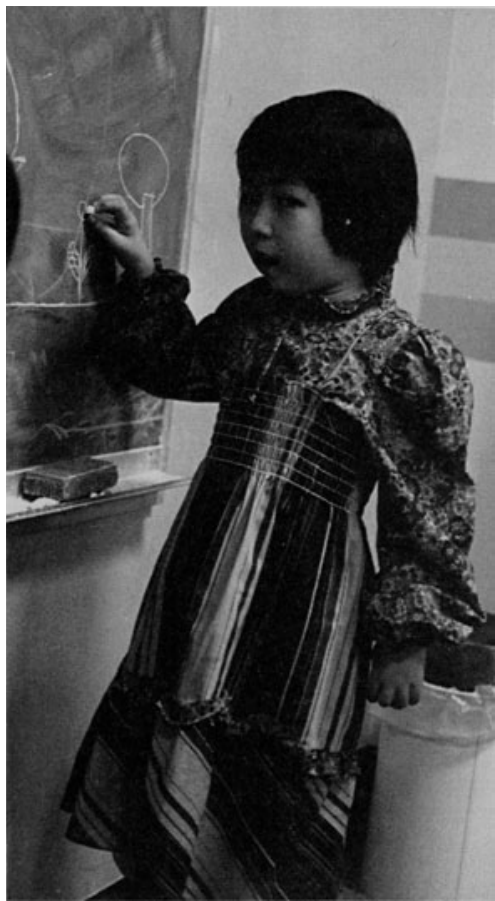

B

Figure 3.52. Patient at the time she was first seen (A) and 3 years later (B) following release of iliotibial bands and soft-tissue contractures about the hips and knees (at one operation) followed by $5 \mathrm{~h}$ of physical therapy daily for several months. The mother was taught the regimen during the 3 -week hospitalization and continued intensive therapy at home. Within 6 months, the child was able to attend a regular public school (despite the language barrier), and to walk without any mechanical aid. The only medicine she received was aspirin. Total hip and knee replacements were performed 10 years later.

what he or she cannot do. The interdisciplinary team exudes optimism, helps to break communication barriers between the primary physician and the family, and contributes, by example to the family, a sense of buoyancy and control over the disease.

\section{Getting Started in the Morning}

Gelling and stiffness make early morning one of the worst times of day for the arthritic child. Arising at 6:00 A.M., taking the medicine, then reading in bed or getting a bit more sleep, followed by a warm bath or shower help in getting started. A simple set of limbering-up exercises is performed, and the child assumes straight posture in front of the mirror. Later in the day, the detailed exercise program is continued. 


\section{The Specific Exercise Program}

If pain is severe, heat in the form of hot baths or local application of towels or soaks may be helpful. The maintenance of motion is achieved by putting every joint of the body, whether involved or uninvolved, through a full range of motion using positions that require as little resistance as possible. The child and parent are shown how to compare normal joint motion with that of affected joints, how to examine the joints, and the visual clues of joint involvement.

A detailed, individually written program is provided to each family as they are taught the exercises. After the program has been taught, it is performed by the child and parent with praise and suggestions from the therapist. This is reviewed and reinforced at several subsequent visits to the rheumatologist. The optimal arrangement is for the physical and occupational therapists to be present in the clinic or office to work with the patients at the regularly scheduled visits or to visit the patients in their homes. ${ }^{559} \mathrm{We}$ try to avoid separate visits to the therapist.

Although gentle passive stretching exercises are used, active and resistance exercises, especially with resistance applied by the child himself, are more effective. Strength of weak muscles may be improved with muscle-tensing exercises that do not involve joint motion (isometric exercises). Many exercises may be performed while in school or in front of the television.

Gravity is used when possible to prevent or correct deformity. Watching TV in the prone position helps to prevent hip-flexion contractures. Children are also encouraged to sleep in this position.

Sleeping splints may be useful as a means of increasing joint mobility (extension) in the wrists and sometimes the knees. Splints must be adjusted and frequently modified; they meet with a fair amount of resistance from both child and family and are only useful when accepted by both as part of an ego-building program. If physical modes of therapy become a source of conflict between parent and child, either the conflict has to be resolved with a psychological approach or the "therapy" will be unsuccessful or even countertherapeutic.

A heel lift on a short leg (usually the uninvolved leg in asymmetrical disease) will help correct or prevent a flexion contracture in the affected, more rapidly growing knee. Persistent leg length discrepancy may result in relative quadraceps atrophy. ${ }^{561}$

Swimming is an excellent exercise, and our families are encouraged to have pools if possible. The buoyant effect of the water allows large weightbearing joints to be moved without force; most children enjoy swimming, and the pool attracts friends and is ego-rewarding for our patients. Other coordinated activities such as ball playing or bicycle riding also provide muscle strengthening and ego-rewarding social opportunities. The disease may set limits on the child's performance, but we do not set any limits whatsoever. We also do not prescribe rest; these children naturally rest more than healthy 
children. Any limitation is inevitably countertherapeutic. Normal childhood is the best therapy. Supplementing normal activities with professionally delivered services can make a great contribution to the patient's welfare; substituting professionally delivered services for being busy with normal activities is relatively useless. ${ }^{562}$ Preventing children from doing things for themselves, developing motor skills, and thus achieving independence and autonomy leads to feelings of helplessness and anger that are countertherapeutic. No exercise program can equal the activities of daily living.

\section{Surgery in JRA}

Pediatric orthopedic surgery requires special understanding of children and growing bones. Juvenile rheumatoid arthritis creates additional special problems. Prolonged immobilization results in atrophy of bone and muscle. When a child has borderline function, immobilization associated with surgery or with treatment of traumatic fracture from a fall may be enough to make the child a bed-chair invalid. Months or years of effort may be required just to get the child back to where he started.

Thus, surgery is not to be lightly embarked upon and should only be performed by experienced operators in pediatric rheumatology centers. Most reports of surgery in JRA refer to adults who have had JRA since childhood, not to children. The subject of surgery in JRA has been thoroughly reviewed by Arden and Ansell, and the reader is referred to their excellent monograph. 268

\section{"Prophylactic" Surgery}

Synovectomy as a means of preventing rather than relieving disability has been tried and abandoned twice in the history of rheumatology. Its value has not been demonstrated. Synovectomy of the knee often relieves pain but is rarely if ever required for that purpose in children. There are few, if any, benefits ${ }^{563-564 a}$ and, if performed, they should be done by arthroscopic synovectomy. ${ }^{565}$

\section{Cosmetic Surgery}

The small mandible caused by JRA troubles many teenagers and is relatively easily corrected with only a few days of hospitalization (Fig. 3.29). The particular surgical procedures must be individualized by experienced physicians. In very rare cases, temporomandibular joint surgery is needed in youngsters who cannot adequately open the mouth.

Some girls wish cosmetic correction of finger deformities. This can be successfully achieved with relative ease, but function does not improve and may even be somewhat reduced. 


\section{Surgery to Relieve Nerve Compression at the Wrist}

In rare individuals, extensive tenosynovitis at the wrist may threaten nerve function; simple releases may be performed to prevent serious loss of function. We have not seen children who required wrist surgery to prevent tendon rupture or to relieve flexion contractures in dysfunctional positions, although others report experience with such procedures.

\section{Corrective and Restorative Surgery}

Soft-tissue release operations, débridement and therapeutic synovectomy are useful in selected instances in JRA (Fig. 3.52). In our experience, these procedures have only been necessary in children with flexion contractures of the hips and knees, accelerated by sitting in wheelchairs, who were bedridden when we first saw them. In these patients, multiple surgical procedures performed at one time may be an essential part of a massive program aimed at getting the child ambulatory again. This goal can then be achieved with a 7-day-per-week, 5-h-per-day rehabilitation program (Fig. 3.53). However, success requires hard work by a well-coordinated, bigcenter, experienced pediatric rehabilitation team. Detailed surgical techniques and anesthetic precaution are reviewed elsewhere. ${ }^{268}$ Benefits tend to be short-lived. 566

\section{Total Joint Replacement}

Experience with hip replacement in young adults who have had destruction of the hips during childhood has been excellent both in our experience and in other centers. ${ }^{567-572}$ The roentgen appearance of the hips should not be used as the criterion for surgery since some patients function well despite an awful radiographic appearance (Figs. 3.54 and 3.55). Replacement of elbows, shoulders, and knees has not yet achieved success equal to hip implants. However, results improve each year. ${ }^{573,574}$

We urge our patients to wait as long as possible before having any joint replacement procedures. The advantage of waiting until the epiphyses close is obvious. In addition, we do not know how long these prosthetic joints are likely to last. Recent experience shows constant technical improvement in implant materials, design, and procedures. Our conservative approach is supported by increasing evidence of potential loss of seemingly fine early prosthetic repair. Early acetabular loosening and radiolucencies predictive of early failure and/or significant heterotopic bone formation are now reported in $15 \%$ of hip replacements in young arthritic patients even in the most experienced clinics (Fig. 3.56), and 15-year hip survival is $70 \% .{ }^{568,572}$ However, we do not allow children to become bed/chair bound; the lives of these youngsters have been transformed by total hip replacement. 570 

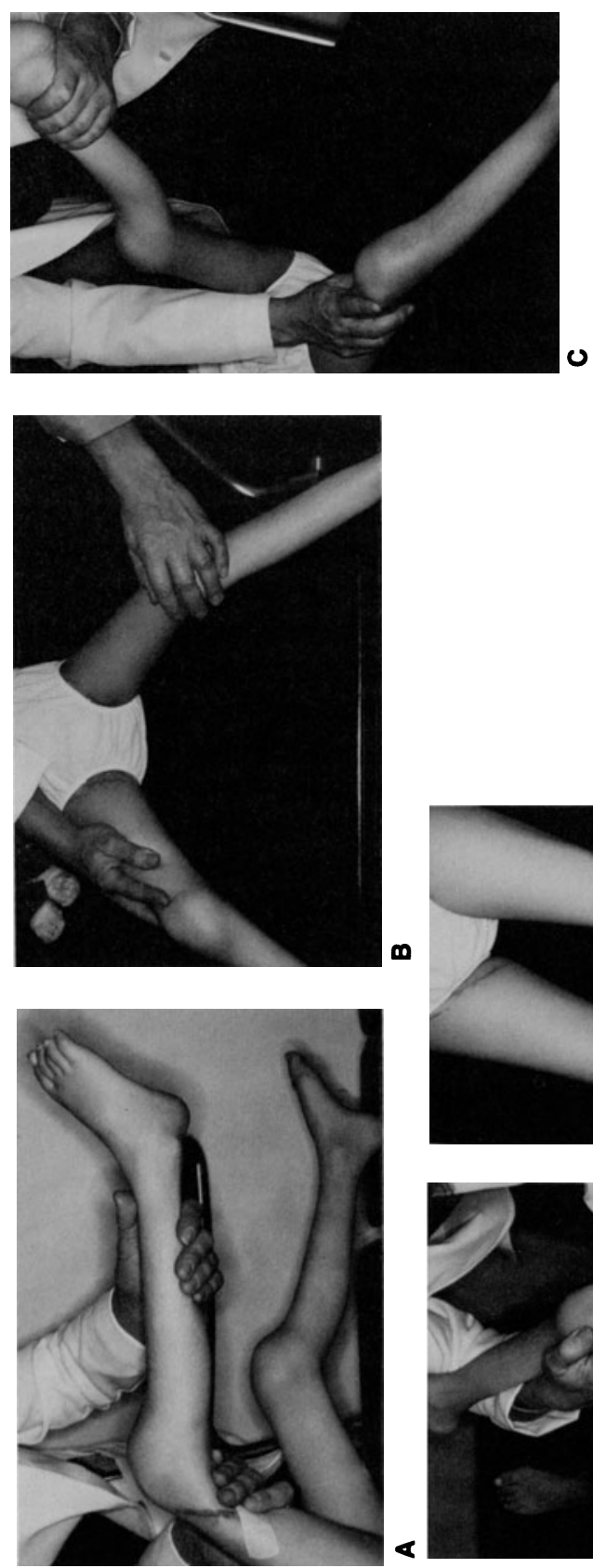

m
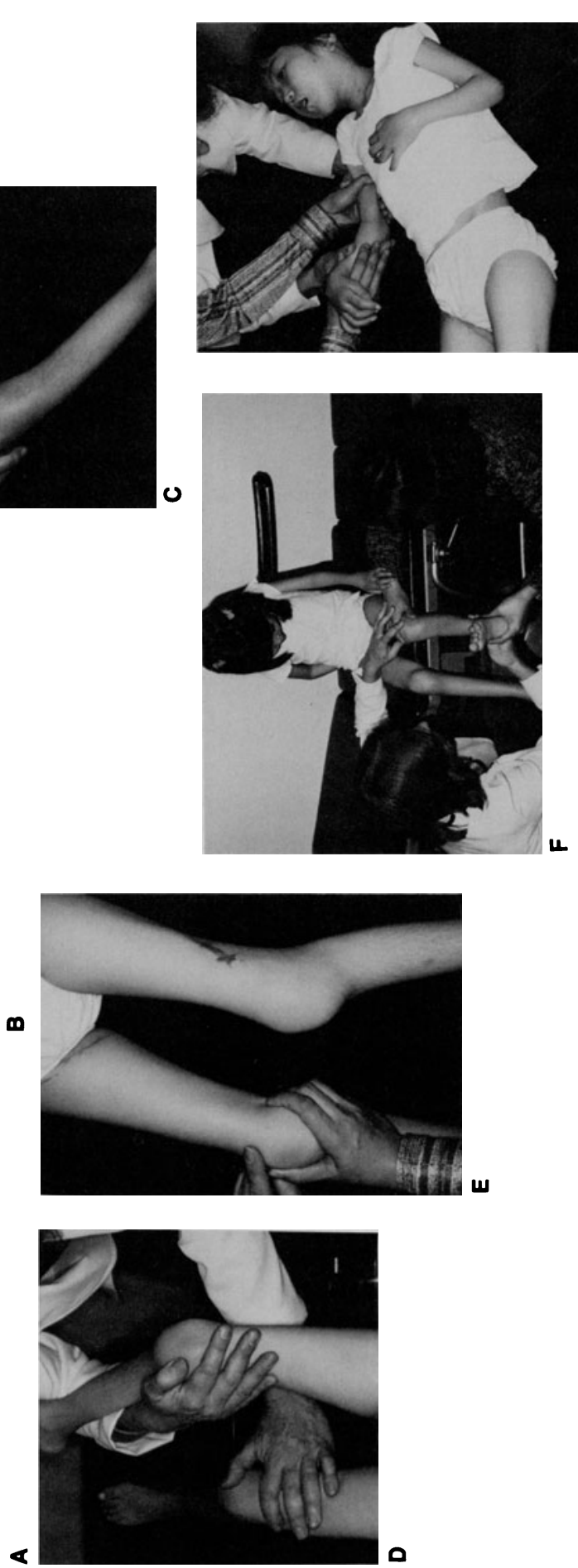

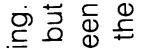

든 등음

Ф

क 응

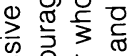

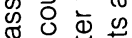

$\Omega$ 品

है 응 ฮ

品

든 은 엉흥 0 亲 壳 S) 응

号

는 은곤

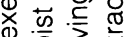

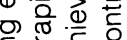

둥

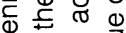

壱我

辰要

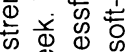

i)

夜

$\vec{\varepsilon} \approx$

잉 30

สิ

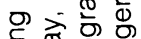

등 잉 인

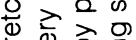

के 원

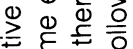

क

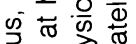

은 을 즘 훙

응

$>0$.

흥 홍

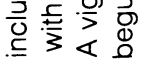

पू है

ह 응 $\frac{\infty}{0}$

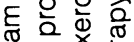

交造

O工

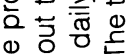

㐘

잉ㅇㅇㅇ

ब

षै己े

กิ

लं क्ष

ఏ هั

可过 

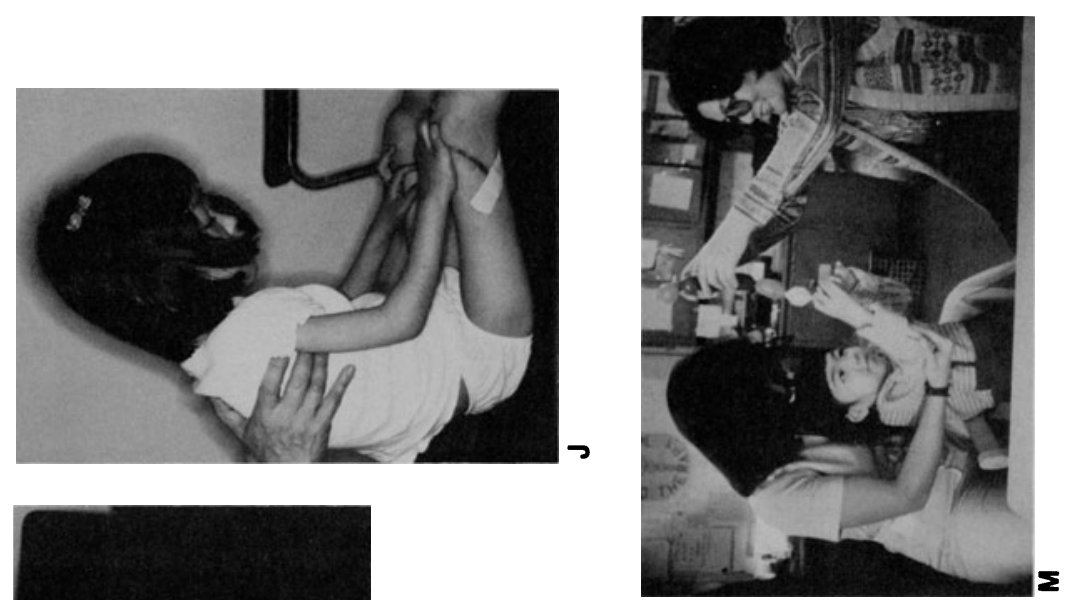

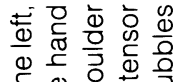
这 प의 क

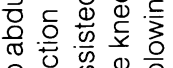
오원

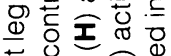

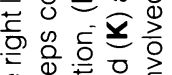

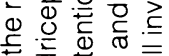
ర్ర 궁

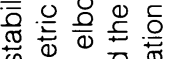

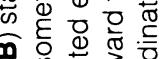

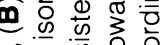
든 क 0 क 产 ()

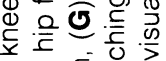

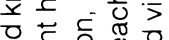

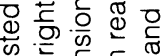

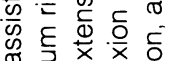
क
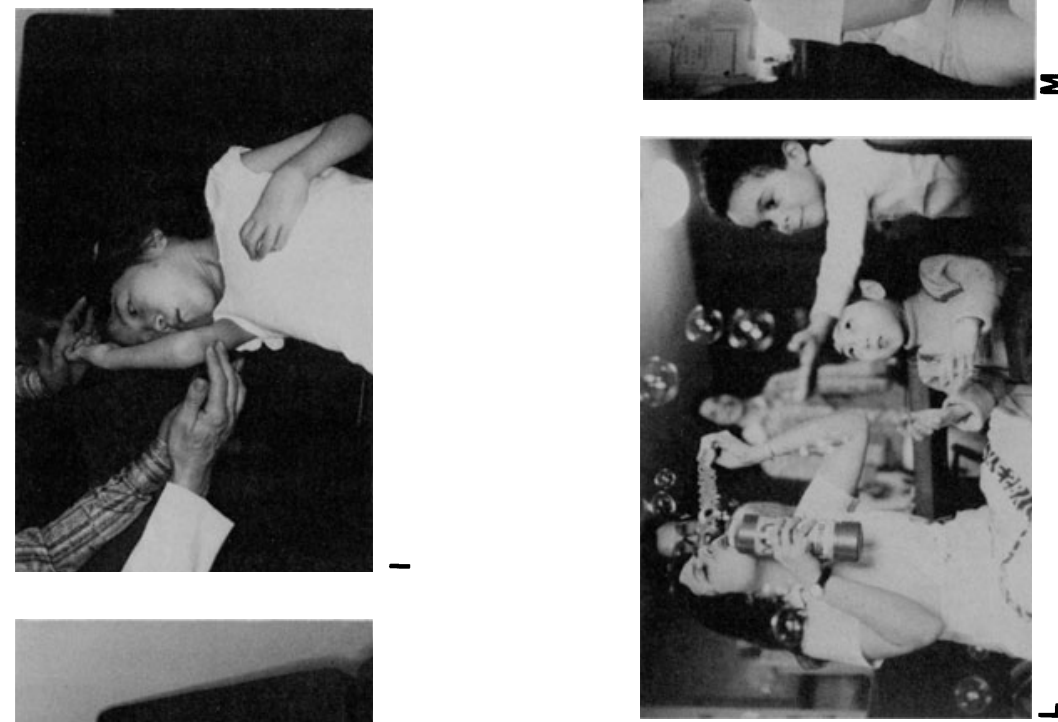

Q : उ

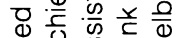
잉

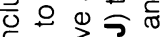
을 है

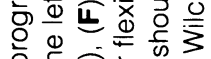
을 क 으은 응

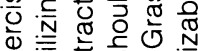

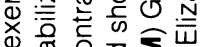

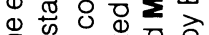
Ф क 00

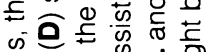

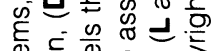

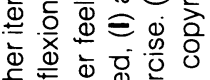

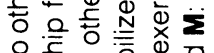
$\circ$ 응

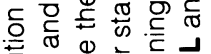
部

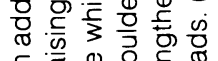

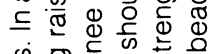

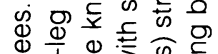
ष्य 为志吉

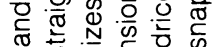

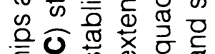




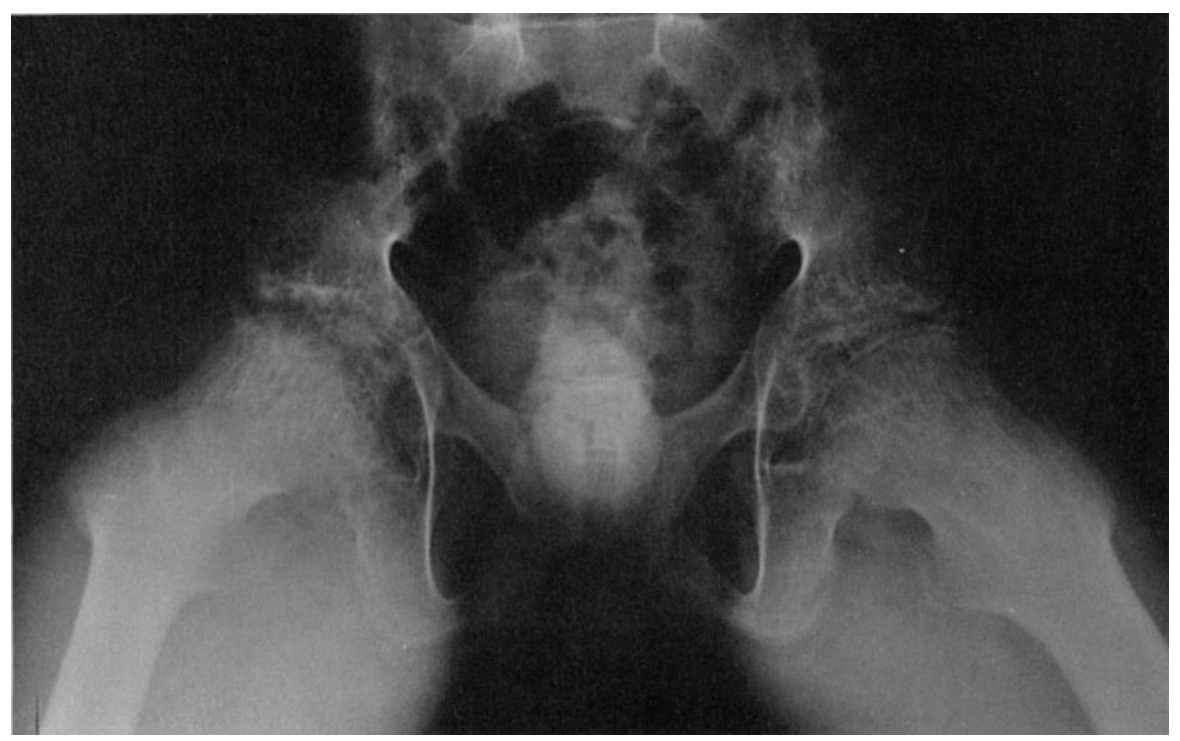

Figure 3.54. Radiograph of the hips in a boy with HLA-B27-associated spondyloarthritis.

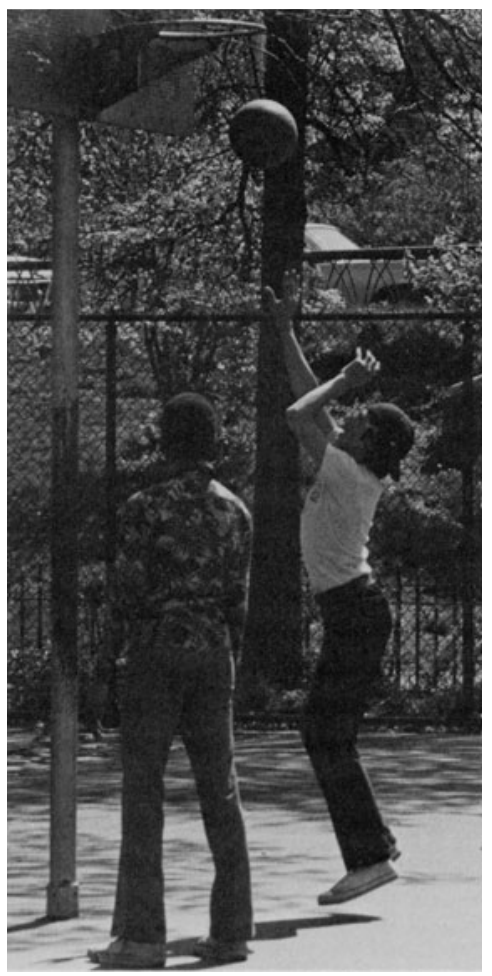

Figure 3.55. Despite the severe radiographic changes (Fig. 3.54), this boy could unexpectedly play basketball and was on his neighborhood team. Although he will ultimately require hip replacement, it has not yet been necessary ( 5 years after this photograph was taken). 

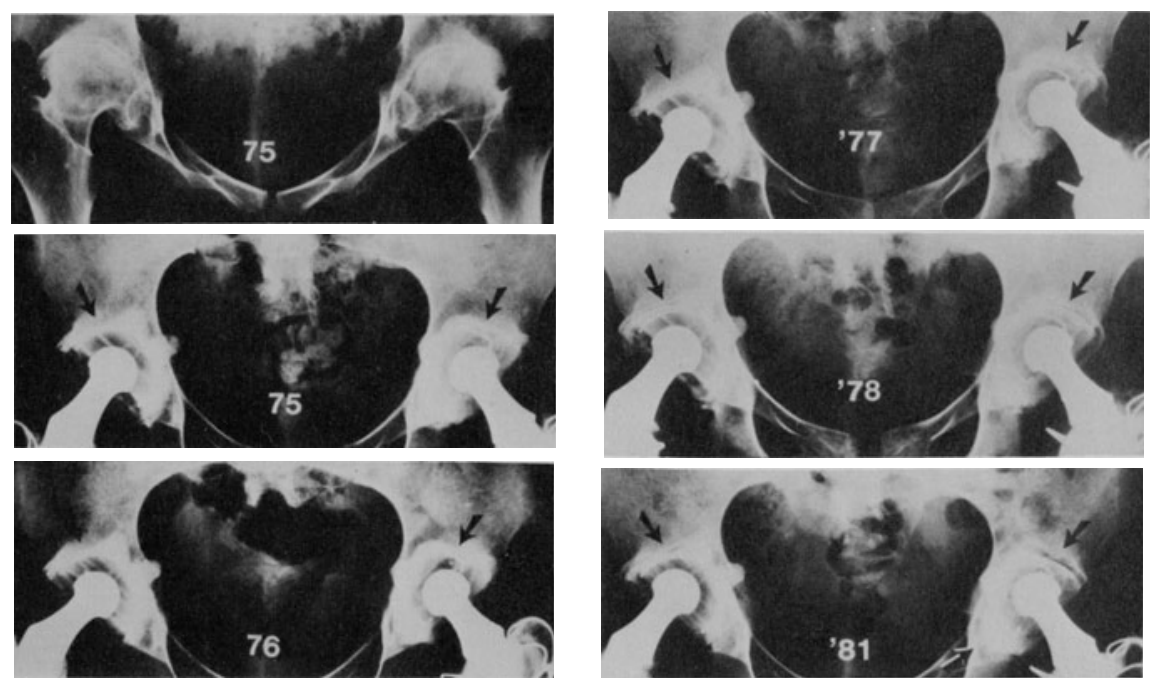

Figure 3.56. Pre- and postoperative radiograph of the hips (1975) in a college girl with long-standing JRA who had no hip abduction and desired hip replacement in order to be able to abduct the hips tor normal sexual function. Bilateral total hip replacements were well tolerated (in two steps; one hospital admission), and the patient has no pain and is delighted with the result. However, from 1975 to 1981 , as shown by the arrows, a progressively increasing "lucency" (interface) is seen in the films. This rapid demarcation occurs in $15 \%$ of young JRA patients following total hip replacement and may be a permanent obstacle to achieving a permanent fixation of the prosthesis to bone in young individuals. It is thought to be due to hyperelasticity of the bone in young patients.

\section{References}

1. JRA Criteria Subcommittee. Criteria for classification of juvenile rheumatoid arthritis. Bull Rheum Dis 25:712-719, 1973.

2. Cassidy JT, Levinson JE, Brewer EJ Jr: The development of classification criteria for children with juvenile rheumatoid arthritis. Bull Rheum Dis 38, \#6:1-7, 1989.

3. Calabro JJ, Marchesano JM: The early natural history of juvenile rheumatoid arthritis. Med Clin North Am 52:567-59l, 1968.

4. Schaller JG, Wedgwood RJ: Juvenile rheumatoid arthritis: A review. Pediatrics 50:940-953, 1972.

5. Schaller JG: Juvenile rheumatoid arthritis. Arthritis Rheum 20:165-170, 1977.

6. Stillman JS, Barry PE: Juvenile rheumatoid arthritis. Arthritis Rheum 20:171175, 1977.

7. Fink CW: Patients with JRA: A clinical study. Arthritis Rheum 20:183-184, 1977.

8. Hanson V, Kornreich HK, Bernstein B, et al.: Three subtypes of juvenile rheumatoid arthritis. Arthritis Rheum 20:184-186, 1977. 
9. Levinson JE, Balz GP, Hess EV: Report of studies on juvenile arthritis. Arthritis Rheum 20:189-190, 1977.

10. Suciu-Foca N, Jacobs JC, Khan R, et al.: Predisposition to juvenile arthritis: genetic aspects. In Moore $\mathrm{T}$ (ed.) Report of the Ross Conference on Pediatric Research. Xanadu, Bahamas, December 3, 1979, Ross Laboratories, Columbus, OH, 1981 pp. 72-75.

11. Baum J, Baum ER: Classics from medical literature: George Frederick Still and his account of childhood arthritis - a reappraisal (with replication of the original monograph On a Form of Chronic Joint Disease in Children). Am J Dis Child 132:192-200, 1978.

12. Green WT: Monarticular and pauciarticular arthritis in children. JAMA 115:2023-2024, 1940.

13. Brewer EJ, Bass J, Baum J, et al.: Current proposed revision of JRA criteria. Arthritis Rheum 20:195-199, 1977.

14. Cassidy JT, Levinson JE, Bass JC, et al.: A study of Classification Criteria for a Diagnosis of Juvenile Rheumatoid Arthritis. Arthritis Rheum 29:274-281, 1986.

15. Bywaters EGL: Still's disease in the adult. Ann Rheum Dis 30:121-133, 1971.

16. Arnett FC, Edworthy SM, Bloch DA, et al.: The American Rheumatism Association 1987 Revised Criteria for the Classification of Rheumatoid Arthritis. Arthritis Rheum 31:315-324, 1988.

17. Stastny P, Fink G: Different HLA-D associations in adult and juvenile rheumatoid arthritis. J Clin Invest 63:124-130, 1979.

18. Glass D, Litvin D, Wallace K, et al.: Early onset pauciarticular juvenile rheumatoid arthritis associated with HLA DRw5, iritis, and antinuclear antibody. J Clin Invest 66:426-429, 1980.

19. van Zeben D, Hazes JMW, Zwinderman AH, et al.: Association of HLA-DR4 with a more progressive disease course in patients with rheumatoid arthritis. Results of a followup study. Arthritis Rheum 34:822-830, 1991.

20. Kunnamo I, Kallio P, Pelkonen P, Hovi T: Clinical signs and laboratory tests in the differential diagonsis of arthritis in children. Am J Dis Child 141:34-40, 1987.

21. Giannini EH, Brewer EJ: Poor correlation between the erythrocyte sedimentation rate and clinical activity in juvenile rheumatoid arthritis. Clin Rheumatol 6:197-201, 1987.

2la.Schwarz-Eywill M, Heilig B, Bauer H, et al.: Evaluation of serum ferritin as a marker for adult Still's disease activity. Ann Rheum Dis 51:683-685, 1992.

22. Eichenfield AH, Athreya BH, Doughty RA, Cebul RD: Utility of rheumatoid factor in the diagonsis of juvenile rheumatoid arthritis. Pediatrics 78:480-484, 1986.

23. Moore TL, Osborn TG, Weiss TD, et al.: Autoantibodies in juvenile arthritis. Semin Arthritis Rheum 13:329-336, 1984.

24. Schaller JG, Johnson GD, Holborow EJ, et al.: The association of antinuclear antibodies with the chronic iridocyclitis of juvenile rheumatoid arthritis (Still's disease). Arthritis Rheum 17:409-416, 1974.

25. Monestier M, Losman JA, Fasy TM, et al.: Antihistone antibodies in antinuclear antibody-positive juvenile arthritis. Arthritis Rheum 33:1836-1841, 1990.

26. Leak AM: Autoantibody profile in juvenile chronic arthritis. Ann Rheum Dis 47:178-182, 1988. 
27. Bergroth V, Konttinen YT, Pelkonen P, et al.: Synovial fluid lymphocytes in different subtypes of juvenile rheumatoid arthritis. Arthritis Rheum 31:780783, 1988.

28. Burdette S, Schwartz RS: Current concepts: immunology idiotypes and idiotypic networks. N. Eng J Med 317:219-224, 1987.

29. Key LL Jr, Hoch S, Cairns L, et al.: Monocyte bone degradation: in vitro analysis of monocyte activity in patients with juvenile rheumatoid arthritis. J Pediatr 108:405-409, 1986.

30. Pauls JD, Silverman E, Laxer RM, Fritzler MJ: Antibodies to histones H1 and $\mathrm{H} 5$ in sera of patients with juvenile rheumatoid arthritis. Arthritis Rheum 32:877-83, 1989.

31. Moore TL, Osborn TG, Dorner RW: Cross-reactive antiidiotypic antibodies against human rheumatoid factors from patients with juvenile rheumatoid arthritis. Arthritis Rheum 32:699-705, 1989.

32. Tsokos GC, Inghirami G, Pillemer SR, et al.: Immunoregulatory aberrations in patients with polyarticular juvenile rheumatoid arthritis. Clin Immunol Immunopathol 47:62-74, 1988.

33. Miller JJ III, Olds LC, Silverman ED, et al.: Different patterns of C3 and C4 activation in the varied types of juvenile arthritis. Pediatr Res 20:1332-1337, 1986.

34. Silverman ED, Somma Catherine, Khan MM, et al.: Abnormal T suppressor cell function in juvenile rheumatoid arthritis. Arthritis Rheum 33:205-211, 1990.

35. Moore TL, Osborn TG, Dorner RW: 19S IgM Rheumatoid factor-7S IgG rheumatoid factor immune complexes isolated in sera of patients with juvenile rheumatoid arthritis. Pediatr Res 20:977-981, 1986.

36. Goldenberg DL: Infectious arthritis complicating rheumatoid arthritis and other chronic rheumatic disorders. Arthritis Rheum 32:496-502, 1989.

37. Baldassare AR, Chano F, Zuckner J: Markedly raised synovial fluid leucocyte counts not associated with infectious arthritis in children. Ann Rheum Dis 37:404-409, 1978.

38. Jacobs JC, Philips PE, Johnston AD: Needle biopsy of the synovium of children. Pediatrics 57:696-701, 1976.

39. Sherman MS: The non-specificity of synovial reactions. Bull Hosp Joint Dis 12:110-125, 1951 .

40. Wynne-Roberts CR: Anderson CH, Turano AM, Baron M. Light and electronmircoscopic findings of juvenile rheumatoid arthritis synovium: comparison with normal juvenile synovium. Semin Arthritis Rheum 7:287-302, 1978.

41. Konttinen YT, Bergroth V, Kunnamo I, Haapasaari J: The value of biopsy in patients with monarticular juvenile rheumatoid arthritis of recent onset. Arthritis Rheum 29:47-53, 1986.

42. Grokoest AW, Snyder AI, Schlaeger R: Juvenile Rheumatoid Arthritis, Little, Brown, Boston, 1962.

43. Martel W, Holt JF, Cassidy JT: Roentgenologic manifestations of juvenile rheumatoid arthritis. Am J Roentgenol Radium Ther Nucl Med 88:400-423, 1962.

44. Poznanski AK, Glass RBJ, Feinstein KA, et al.: Magnetic resonance imaging in juvenile rheumatoid arthritis. Int Pediatr 3:304-310, 1988.

45. Senac MO, Deutsch D, Bernstein BH, et al.: MR Imaging in juvenile rheuma- 
toid arthritis. AJR 150:873-878, 1988.

46. Stoller DW, Genant HK: Magnetic resonance imaging of the knee and hip. Arthritis Rheum 33:441-449, 1990.

47. Sartoris DJ: Magnetic resonance imaging of the musculoskeletal system. J Musculoskel Med 7:29-45, 1990.

48. Towner SR, Michael CJ Jr, O'Fallon WM, Nelson AM: The epidemiology of juvenile arthritis in Rochester, Minnesota 1960-1979. Arthritis Rheum 26:1208-1213, 1983.

49. Kunnamo I, Kallio P, Pelkonen P: Incidence of arthritis in urban Finnish children: A prospective study. Arthritis Rheum 29:1232-1238, 1986.

50. Petty RE: Epidemiology of juvenile rheumatoid arthritis. In Miller JJ III (ed.) Juvenile Rheumatoid Arthritis. PSG, Littleton, MA 1979, pp. 11-18.

51. Gewanter HL, Roghmann KJ, Baum J: The prevalence of juvenile arthritis Arthritis Rheum 26:599-603, 1983.

52. Laaksonen A: A prognostic study of juvenile rheumatoid arthritis. Acta Paediatr Scand [Suppl.] 166, 1966.

53. Kredich DW: Polyarticular juvenile rheumatoid arthritis. In Miller JJ III (ed.) Juvenile Rheumatoid Arthritis. PSG, Littleton, MA, 1977, pp. 121-134.

54. Clemens LE, Albert E, Ansell BM: Sibling pairs affected by chronic arthritis of children: Evidence for a genetic predisposition. J Rheumatol 12:108-113, 1985.

55. Nepom BS, Malhortra U, Schwarz DA, et al.: HLA and T Cell receptor polymorphisms in pauciarticular-onset juvenile rheumatoid arthritis. Arthritis Rheum 34:1260-1267, 1991.

56. Aaron S, Fraser PA, Jackson JM, et al.: Sex ratio and sibship size in juvenile rheumatoid arthritis kindreds. Arthritis Rheum 28:753-758, 1985.

57. van Kerckhove C, Glass DN: The immunoglobulin supergene family and the polygenic nature of inherited predisposition to rheumatic disease. Arthritis Rheum 30:951-953, 1987.

58. Albert ED, Ansell BM: Immunogenetics of juvenile chronic arthritis. Scand J Rheumatol Suppl. 66:85-91, 1987.

59. Calabro JJ, Marchesano JM: Fever associated with juvenile rheumatoid arthritis. N Engl J Med 276:11-18, 1967.

60. Pizzo PA, Lovejoy FH Jr, Smith DH: Prolonged fever in children: Review of 100 cases. Pediatrics 55:468-473, 1975.

61. Lohr JA, Hendley JO: Prolonged fever of unknown origin. Clin Pediatr 16:768773, 1977.

62. McClung HJ: Prolonged fever of unknown origin in children. Am J Dis Child 124:544-550, 1972.

63. Schaller J: Arthritis as a presenting manifestation of malignancy in children. J Pediatr 81:793-797, 1972.

64. Estrada R, Schaeffer H, Rosenfeld W, et al.: Anhidrotic ectodermal dysplasia. Fever in a neonate. NYS J Med 81:1791-1793, 1981.

65. Case records of the Massachusetts General Hospital. CASE No. 22:1991. N Eng J Med 324:1575-1584, 1991.

66. Lake AM, Oski FA: Peripheral lymphadenopathy in childhood. Am J Dis Child 132:357-359, 1978.

67. Sullivan DB, Cassidy JT, Petty RE: Pathogenic implications of age of onset in juvenile rheumatoid arthritis. Arthritis Rheum 18:251-254, 1975.

68. Ansell BM, Bywaters EGL: Rheumatoid arthritis (Still's disease). Ped Clin 
North Am 10:921-939, 1963.

69. Bujak JS, Aptekar RG, Decker JL, et al.: Juvenile rheumatoid arthritis presenting in the adult as fever of unknown origin. Medicine 52:431-444, 1973.

70. Ohta A, Yamaguchi M, Kaneoka H, et al.: Adult Still's Disease: review of 228 cases from the literature. J Rheumatol 14:1139-1146, 1987.

71. Cush-JJ, Medsger TA Jr, Christy, WC: Adult-onset Still's disease. Clinical course and outcome. Arthritis Rheum 30:186-194, 1987.

72. Reginato AJ, Schumacher HR Jr, Baker DG, et al.: Adult onset Still's disease: experience in 23 patients and literature review with emphasis on organ failure. Semin Arthritis Rheum 17:39-57, 1987.

73. Lindsley CB: Seasonal variation in systemic onset juvenile rheumatoid arthritis. Arthritis Rheum 30:838-839, 1987.

74. Ansell BM, Bywaters EGL: Diagnosis of "probable" Still's disease and its outcome. Ann Rheum Dis 21:253-262, 1962.

75. Isdale IC, Bywaters, EGL: The rash of rheumatiod arthritis and Still's disease. QJ Med 25:377-387, 1956.

76. Calabro JJ, Marchesano JM: Rash associated with juvenile rheumatoid arthritis. J Pediatr 72:611-619, 1968.

77. de Benedetti F, Massa M, Robbioni P, et al.: Correlation of serum interleukin-6 levels with joint involvement and thrombocytosis in systemic juvenile rheumatoid arthritis. Arthritis Rheum 34:1158-1163, 1991.

78. Schlesinger BE, Forsyth CC, White RHR, et al.: Observations on the clinical course and treatment of one hundred cases of Still's disease. Arch Dis Child 36:65-76, 1961.

79. Calabro JJ, Burnstein SL, Staley HL: JRA posing as fever of unknown origin. Arthritis Rheum 20:178-180, 1977.

79a.Schneider R, Lang BA, Reilly BJ, et al.: Prognostic indicators of joint destruction in systemic-onset juvenile rheumatoid arthritis. J Pediatr 120:200-5, 1992

80. Barber FA, Roach JW: Torticollis: A presentation of eosinophilic granuloma: A case report. Orthopedics 9:1237-1239, 1986.

81. Steinberg GG: Osteomyelitis of the rib presenting as painful torticollis: Case report. J Bone Joint Surg 61-A:614-616, 1979.

82. Schaller JG: The diversity of JRA. Arthritis Rheum 20 (Suppl):52-63, 1977.

83. Rachelefsky GS, Kar NC, Coulson A, et al.: Serum enzyme abnormalities in juvenile rheumatoid arthritis. Pediatrics 58:730-736, 1976.

84. Halla JT, Koopman WJ, Fallahi S, et al.: Rheumatoid myositis. Clinical and histologic features and possible pathogenesis. Arthritis Rheum 27:737-743, 1984.

85. Schaller JG, Beckwith B, Wedgwood RJ: Hepatic involvement in juvenile rheumatoid arthritis. J Pediatr 77:203-209, 1970.

86. Brewer EJ, Giannini E, Person D: Juvenile Rheumatoid Arthritis 2nd edition, Saunders, Philadelphia, 1982.

87. Ohta A, Matsumoto Y, Ohta T, et al.: Still's disease associated with necrotizing lymphadenitis (Kikuchi's disease): Report of 3 cases. J Rheumatol 15:981-983, 1988 (see also: Correspondence: 17:568-569, 1990).

88. Wang FM, Wertenbaker C, Behrens MM, Jacobs JC: Acquired Brown's syndrome in children with juvenile rheumatoid arthritis. Ophthalmology 91:2326, 1984.

89. Yousefzadeh DK, Fishman PA: The triad of pneumonitis, pleuritis, and peri- 
carditis in juvenile rheumatoid arthritis. Pediatr Radiol 8:147-150, 1979.

90. Brewer E: Juvenile rheumatoid arthritis-cardiac involvement. Arthritis Rheum 20:231-236, 1977.

91. Miller JJ III: Carditis in JRA. In Miller JJ III (ed.) Juvenile Rheumatoid Arthritis. PSG, Littleton, MA, 1979, pp. 165-173.

92. Yancey CL, Doughty RA, Cohlan BA, Athreya BH: Pericarditis and cardiac tamponade in juvenile rheumatoid arthritis. Pediatrics 68:369-373, 1981.

93. Lietman PS, Bywaters EGL: Pericarditis in juvenile rheumatoid arthritis. Pediatrics 32:855-860, 1963.

94. Svantesson H, Bjorkhem G, Elborgh R: Cardiac involvement in juvenile rheumatoid arthritis: a follow-up study. Acta Paediatr Scand 72:345-350, 1983.

95. Smith WG: Coxsackie B myopericarditis in adults. Am Heart J 80:34-46, 1970.

96. Tilzey AJ, Signy M, Banatvala JE: Persistent coxsackie B virus specific IgM response in patients with recurrent pericarditis. Lancet 1:1491-1492, 1986.

97. Soutar CA: Unusual case of viral pericarditis. Lancet 1:498, 1971.

98. Rahal JJ, Millian SJ, Noriega ER: Coxsackie virus and adenovirus infection: Association with acute febrile and juvenile rheumatoid arthritis. JAMA 235:2496-2501, 1976.

99. Adler R, Takahashi M, Wright HT: Acute pericarditis associated with hepatitis B infection. Pediatrics 61:716-719, 1978.

100. Wong KF, Chan JKC, Chan CH: Psittacosis-Associated hemophagocytic syndrome. Am J Med 91:204-205, 1991.

101. Morris JA, Adamson AR, Holt PJL, Davson J: Still's disease and the virusassociated haemophagocytic syndrome. Ann Rheum Dis 44:349-353, 1985.

102. Heaton DC, Moller PW: Case report: Still's disease associated with Coxsackie infection and haemophagocytic syndrome. Ann Rheum Dis 44:341-344, 1985.

103. Hurst NP, Martkynoga AG, Nuki G, et al.: Coxsackie B infection and arthritis. Br Med J 286:580, 1983.

104. Roberts-Thomson PJ, Ahern MJ, Southwood TR, et al.: Adult onset Still's Disease or Coxsackie Polyarthritis? Aust NZ J Med 16:509-511, 1986.

105. Fraser KJ, Clarris BJ, Muirden KD, et al.: A persistent adenovirs type 1 infection in synovial tissue from an immunodeficient patient with chronic, rheumatoid-like polyarthritis. Arthritis Rheum 28:455-458, 1985.

106. Denman AM: A viral aetiology for juvenile chronic arthritis. J Rheum 27:169175, 1988.

107. Delgado EA, Petty RE, Malleson PN, et al.: Aortic valve insufficiency and coronary artery narrowing in a child with polyarticular juvenile rheumatoid arthritis. J Rheumatol 15:144-147, 1988.

108. Singleton EB, Wagner ML: Radiographic Atlas of Pulmonary Abnormalities in Children. Saunders, Philadelphia, 1971, p. 240.

109. Athreya BH, Doughty RA, Bookspan M, et al.: Pulmonary manifestations of juvenile rheumatoid arthritis. A report of eight cases and review. Clin Chest Med 1:361-374, 1980.

110. Lovell D, Lindsley C, Langston C: Lymphoid interstitial pneumonia in juvenile rheumatoid arthritis. J Pediatr 105:947-950, 1984.

111. O'Brodovich HM, Way RC, Andrew M, Dent PB: Nonivasive diagnosis of pulmonary hemorrhage in rheumatoid arthritis. Pediatrics 72:720-723, 1983.

112. Wagener JS, Taussig LM, DeBenedetti G, et al.: Pulmonary function in juvenile rheumatoid arthritis. J Pediatr 99:108-110, 1981. 
113. Padeh S, Laxer RM, Silver MM, Silverman ED: Primary pulmonary hypertension in a patient with systemic-onset juvenile arthritis. Arthritis Rheum 34:1575-1679, 1991.

114. Jacobs JC, Hui RM: Cricoarytenoid arthritis and airway obstruction in juvenile rheumatoid arthritis. Pediatrics 59:292-294, 1977.

115. Jan JE, Hill RH, Low MD: Cerebral complications in juvenile rheumatoid arthritis. Can Med Assoc J 107:623-625, 1972.

116. Sievers K, Nisshla M, Sievers UM: Cerebral vasculitis visualized by angiography in juvenile rheumatoid arthritis simulating brain tumor. Acta Rheum Scand 14:222-232, 1968.

117. O'Connor D, Bernstein B, Hanson V, et al.: Disease of central nervous system in juvenile rheumatoid arthritis (JRA), abstracted. Arthritis Rheum 23:727, 1980.

118. Anttila R: Renal involvement in juvenile rheumatoid arthritis. A clinical and histopathological study. Acta Paediatr Scand [Suppl.] 227, 1972.

119. Stapleton FB, Hanissian AS, Miller LA: Hypercalciuria in children with juvenile rheumatoid arthritis: association with hematuria. J Pediatr 107:235-239, 1985.

120. Reed A, Haugen M, Pachman LM, Langman CB: Abnormalities in serum osteocalcin values in children with chronic rheumatic diseases. J Pediatr 116:574-580, 1990.

121. Levy M, Prieur A-M, Gubler M-C, et al.: Renal involvement in juvenile chronic arthritis: clinical and pathologic features. Am J Kidney Dis 9:138-146, 1987.

122. Malleson PN, Lockitch G, MacKinnon M, et al.: Renal disease in chronic arthritis of childhood. A study of urinary $\mathrm{N}$-acetyl-8-glucosaminidase and B2microglobulin excretion. Arthritis Rheum 33:1560-1566, 1990.

123. Schnitzer TJ, Ansell BM: Amyloidosis in juvenile chronic polyarthritis. Arthritis Rheum 20:245-252, 1977.

124. Youyfouka O, David J, Ansell BA, et al.: Mortality and morbidity of a cohort of juvenile arthritis patients with amyloidosis. Arthritis Rheum 335:144, 1990.

125. Baum J. Gutowska G: Death in juvenile rheumatoid arthritis. Arthritis Rheum 20:253-255, 1977.

126. Duston MA, Skinner M, Shirahama T, Cohen AS: Diagnosis of amyloidosis by abdominal fat aspiration: analysis of four years' experience. Am J Med 82:412414, 1987.

127. Cohen AS, Skinner M: New frontiers in the study of amyloidosis. N Engl J Med 323:542-543, 1990.

127a.Hawkins PN, Richardson S, David J, et al.: Monitoring amyloidosis in juvenile rheumatoid arthritis with serum amyloid $\mathrm{P}$ component scintography. Arthritis Rheum 34:S124, 1991.

128. Rostropowicz-Denisiewicz K, Romicka AM, Berkan E, Luft S: The course of amyloidosis in juvenile chronic arthritis with immunoglobulin deficiency. Scand J Rheumatol. Suppl 67:27-29, 1988.

129. Escalante A, Ehresmann GR, Quismorio FP Jr.: Regression of reactive systemic amyloidosis due to ankylosing spondylitis following the administration of colchicine. Arthritis Rheum 34:920-922, 1991.

130. Gall EP, Goldberg M. Editorial. The safety of treating rheumatoid arthritis with aspirin and analgesic nephropathy in 1981: Which drug is responsible? JAMA 247:63-65, 1982. 
131. Wortmann DW, Kelsch RC, Kuhns L, et al.: Renal papillary necrosis in juvenile rheumatoid arthritis. J Pediatr 97:37-40, 1980.

132. Allen RC, Petty RE, Lirenman DS, et al.: Renal papillary necrosis in children with chronic arthritis. Am J Dis Child 140:20-22, 1986 (see also editorial pp. 16-17).

133. Koerper MA, Stempel DA, Dallman PR: Anemia in patients with juvenile rheumatoid arthritis. J Pediatr 92:930-933, 1980.

134. Gross SJ, Stuart MJ, Swender PT, et al.: Malabsorption of iron in children with iron deficeincy. J Pediatr 88:795-799, 1976.

135. Vichinsky E: Anemia associated with rhenumatoid arthritis. J Pediatr 94:678, 1979.

136. Hochbery MC, Arnold CM, Hogans BB, Spivak JL: Serum immunoreactive erythropoietin in rheumatoid arthritis: impaired response to anemia. Arthritis Rheum 31:1318-1321, 1988.

137. Maury CPJ, Andersson LG, Teppo A-M, et al.: Mechanism of anaemia in rheumatoid arthritis: demonstration of raised interleukin 1 concentrations in anaemic patients and of interleukin 1 mediated suppression in normal erythropoiesis and proliferation of human erythroleukaemia (HEL) cells in vitro. Ann Rheum Dis 47:972-978, 1988.

138. Dessypris EN, Baer MR, Sergent JS, Krantz SB: Rheumatoid arthritis and pure red cell aplasia. Ann Intern Med 100:202-206, 1984.

139. Rubin RN, Walker BK, Ballas SK, et al.: Erythroid aplasia in juvenile rheumatoid arthritis. Am J Dis Child 132:760-762, 1978.

140. Glader BE: Evaluation of the hemolytic role of aspirin in glucose-6-phosphate dehydrogenase deficiency. J Pediatr 89:1027-1028, 1976.

141. Craft AW, Eastham EJ, Bell JI, et al.: Serum ferritin in juvenile chronic polyarthritis. Ann Rheum Dis 36:271-273, 1977.

142. Vreugdenhil G, Baltus CAM, Van Eijk HG, Swaak AJG: Anaemia of chronic disease: Diagnostic significance of erythrocyte and serological parameters in iron deficient rheumatoid arthritis patients. Br J Rheumatol 29:105-110, 1990.

143. Johansson U, Portinsson S, Akesson A, et al.: Nutritional status in girls with juvenile rheumatoid arthritis. Hum Nutr Clin Nutr 40C:57-67, 1985.

144. Herbert V: The nutritional anemias. Hosp Pract 15(3):65-89, 1980.

145. Winyard PG, Blake DR, Chirico S, et al.: Mechanism of exacerbation of rheumatoid synovitis by total-dose iron-dextran infusion: in-vivo demonstration of iron-promoted oxidant stress. Lancet 1:69-72, 1987 (see also correpondence p. 391).

146. Pincus T, Olsen NJ, Russell IJ, et al.: Multicenter study of recombinant human erythropoietin in correction of anemia in rheumatoid arthritis. Am J Med 89:161-168, 1990.

146a.Fantini F, Gattinara M, Gerloni V, et al.: Severe anemia associated with systemic-onset juvenile rheumatoid arthritis successfully treated with recombinant human erythropoeitin: a pilot study. Arthritis Rheum 35:724-726, 1992.

147. Kuhns JG, Swain LT: Distrubances of growth in chronic arthritis in children. Am J Dis Child 43:1118-1133, 1932.

148. Coss JA Jr, Boots RH: Juvenile rheumatoid arthritis-a study of fifty-six cases with a note on skeletal changes. J Pediatr 29:143-156, 1946.

149. Ansell BM, Bywaters EGL: Growth in Still's disease. Ann Rheum Dis 15:295$319,1956$. 
150. Bernstein BH, Stobie D, Singsen $\mathrm{BH}$, et al.: Growth retardation in juvenile rheumatoid arthritis (JRA). Arthritis Rheum 20:212-216, 1977.

151. Bennett AE, Silverman ED, Miller JJ III, Hintz RL: Insulin-like growth factors I and II in children with systemic onset juvenile arthritis. J Rheumatol 15:655658, 1988.

152. Aitman TJ, Palmer RG, Loftus J, et al.: Serum IGF-I levels and growth failure in juvenile chronic arthritis. Clin Exp Rheumatol 7:557-561, 1989.

153. Sadeghi-Nejad A, Seniro B: Adrenal function, growth and insulin in patients treated with corticoids on alternate days. Pediatrics 43:277-283, 1969.

154. Davies UM, Ansell BM, Woo P: Growth retardation in juvenile chronic arthritis: effect of treatment with biosynthetic human growth hormone. Arthritis Rheum 33:S14, 1990.

155. Rappaport EB, Snoy P, Habig WH, Bright RW: Effects of exogenous growth hormone on growth plate cartilage in rats. Am J Dis Child 141:497-501, 1987.

156. Loeb JN: Corticosteroids and growth. N Engl J Med 295:547-552, 1976.

157. Ansell BM, Bywaters EGL: Alternate-day corticosteriod therapy in juvenile chronic polyarthritis. J Rheum 1:176-186, 1974.

158 Wales JKH, Mllner RDG: Variation in lower leg growth with alternate day steroid treatment. Arch Dis Child 63:981-983, 1988.

159. Richards GE, Pachman LM, Green CC: Symptomatic hypothyroidism in children with collagen disease. J Pediatr 87:82-84, 1975.

160. Collen RJ, Lippe BM, Kaplan SA: Primary ovarian failure, juvenile rheumatoid arthritis, and vitiligo. Am J Dis Child 133:598-600, 1979.

161. Fisher M, Nussbaum M, Abrams CAL, et al.: Diabetes mellitus. Hashimoto's, thyroiditis, and juvenile rheumatoid arthritis. Am J Dis Child 134:93-94, 1980.

162. Pirani CL, Bennett GA: Rheumatoid arthritis: A report of three cases progressing from childhood and emphasizing certain systemic manifestations. Bull Hosp Joint Dis 12:335-367, 1951.

163. Cabane J, Michon A, Ziza J-M, et al.: Comparison of long term evolution of adult onset and juvenile onset Still's disease, both followed up for more than 10 years. Ann Rheum Dis 49:283-285, 1990.

164. Bywaters EGL: Deaths in juvenile chronic polyarthritis. Arthritis Rheum 20:256, 1977.

165. Bernstein B: Death in juvenile rheumatoid arthritis. Arthritis Rheum 20:256257, 1977.

166. Scott JP, Pachman LM, Gerber PS, et al.: Activation of coagulation in systemic JRA (S-JRA). Arthritis Rheum 26:S57, 1983.

167. Mukamel M, Bernstein BH, Brik R, Lehman TJA: The prevalence of coagulation abormalities in juvenile rheumatoid arthritis. J Rheumatol 14:1147-1149, 1987.

168. Sbarbaro JA, Bennett RM: Aspirin hepatotoxicity and disseminated intravascular coagulation. Ann Intern Med 86:183-185, 1977.

169. Jacobs JC, Gorin LJ, Hanissian AS, Simon JL: Consumption coagulopathy after gold therapy for JRA. J Pediatr 105:674-675, 1984.

170. Silverman ED, Miller JJ III, Bernstein B, Shafai T: Comsumption coagulopathy associated with systemic juvenile rheumatoid arthritis. J Pediatr 103:872876, 1983.

171. Pinedo HM, Van de Putte BA, Loeliger EA: Salicylate-induced consumption coagulopathy. Ann Rheum Dis 32:66-68, 1973. 
172. De Vere-Tyndall A, MacAuley D, Ansell BM: Disseminated intravascular coagulation complicating systemic juvenile chronic arthritis ("Still's disease"). Clin Rheumatol 2:415-418, 1983.

173. Kornreich H, Malouf NN, Hanson V: Acute hepatic dysfunction in juvenile rheumatoid arthritis. J Pediatr 79:27-35, 1971.

174. Lipinski J, Meyer R, Kornetsky C, Cohen BM: Adult respiratory-distress syndrome in salicylate intoxication. Lancet 1:1294-1295, 1979.

175. Case Records of the Massachusetts General Hospital: Case 23-1977. N Eng J Med 296:1337-1346, 1977.

176. Merskey C: DIC: Identification and management. Hosp Pract pp. 83-94, December 1982.

177. Sherry DD, Kredich DW: Transient thrombocytopenia in systemic onset juvenile rheumatoid arthritis. Pediatrics 76:600-603, 1985.

178. Hadchouel M, Prieur A-M, Griscelli C: Acute hemorrhagic, hepatic, and neurologic manifestations in juvenile rheumatoid arthritis: possible relationship to drugs or infection. J Pediatr 106:561-566, 1985.

179. Levy J, Pettei MJ, Weitz JI: Dysfibrinogenemia in obstructive liver disease. J Pediatr Gastroenterol Nutr 6:967-970, 1987.

180. Ohaska A, Saito K, Mori S, et al.: Clinicopathologic and therapeutic aspects of angioimmunoblastic lymphadenopathy-related lesions. Cancer 69:1259-1267, 1992.

181. Steinberg AD, Seldin MF, Jaffe ES, et al.: Angioimmunoblastic lymphadenopathy with dysproteinemia. Ann Intern Med 108:575-584, 1988.

182. Buchanan WW, Rooney RJ, Rennie JAN: Aspirin and the salicylates. Clin Rheum Dis 5:499-539, 1979.

183. Levy G, Leonards JR: Urine pH and salicylate therapy. JAMA 217:81, 1971.

184. Lanza FL, Royer GL Jr, Nelson RS: Endoscopic evaluation of the effects of aspirin, buffered aspirin, and enteric coated aspirin on gastric and duodenal mucosa. N Engl J Med 303:136-138, 1980.

185. Vane JR: Inhibitors of prostaglandin, prostacyclin and thromboxane synthesis. In Coceani F, Olley PJ (eds.) Advances in Prostaglandin Research, Vol. 4 Raven Press, New York, 1978, pp. 27-44.

186. Morley J, Bray MA, Gordon D: The action of anti-inflammatory drugs on the lymphocyte-macrophage axis. In Glynn LE, Schlumberger HD (eds.) Experimental Models of Chronic Inflammatory Disease. Springer-Verlag, Berlin, 1977, pp. 376-390.

187. Boardman PL, Hart FD: Clinical measurement of the anti-inflammatory effects of salicylates in rheumatoid arthritis. Br Med J 4:264-268, 1967.

188. Ansell BM: Juvenile chronic arthritis. In Hart FD (ed.) Drug Treatment of the Rheumatic Diseases. Williams and Wilkins. Sydney, 1987, pp. 196-208.

189. Doughty RA, Giesecke L, Athreya B: Salicylate therapy in juvenile rheumatoid arthritis: dose, serum level, and toxicity. Am J Dis Child 134:461-463, 1980.

190. Jacobs JC, Pesce M: Micro-measurement of plasma salicylate in arthritic children. Arthritis Rheum 21:129-132, 1978.

191. Bardare M, Cislaghi GU, Mandelli M, et al.: Value of monitoring plasma salicylate levels in treating juvenile rheumatoid arthritis. Arch Dis Child 53:381-385, 1978.

192. Levy G: Clinical pharmacokinetics of aspirin. Pediatrics 62:867-872, 1978.

193. Graham GG, Champion GD, Day RD, et al.: Patterns of plasma concentrations and urinary excretion of salicylate in rheumatiod arthritis. Clin Pharmacol Ther 
22:410-420, 1977.

194. Wolfe F, Hawley DJ: Remission in rheumatoid arthritis. J Rheumatol 12:245252, 1985.

195. Halla JT, Hardin JG: Salicylate ototoxicity in rheumatoid arthritis: A controlled study. Ann Rheum Dis 47:134-137, 1988.

196. Day RO, Furst DE, Dromogoole SH, et al.: Relationship of serum naproxen concentration to efficacy in rheumatoid arthritis. Clin Pharmacol Ther 31:733$740,1982$.

197. Wilson JT, Brown RD, Kearns GL, et al.: Single-dose, placebo-controlled comparative study of ibuprofen and acetaminophen antipyresis in children. J Pediatr 119:803-811, 1991.

198. Jacobs JC: New Frontiers in Pediatric Rheumatology. World Health Communications, New York, 1986.

199. Brewer EJ, Giannini EH, Barkley E: Gold therapy in the management of juvenile rheumatoid arthritis. Arthritis Rheum 23:404-411, 1980.

200. Roine R, Gentry T, Hernandez-Munoz R, et al.: Aspirin increases blood alcohol concentrations in humans after ingestion of ethanol. JAMA 264:2406-2408, 1990.

201. Jusko WJ, Rose JQ: Monitoring prednisone and prednisolone, In Tognoni G, Latini R, Jusko WJ (eds.) Frontiers in Therapeutic Drug Monitoring. Raven Press, New York, 1980, pp. 153-161.

202. Nelson AM, Conn DL: Glucocorticoids in rheumatic disease. Mayo Clin Proc 55:758-769, 1980.

203. Byron MA, Jackson J, Ansell BM: Effect of different corticosteroid regimens on hypothalamic-pituitary-adrenal axis and growth in juvenile chronic arthritis. JR Soc Med 76:452-457, 1983.

204. Schurmeyer TH, Tsokos GC, Avgerinos PC, et al.: Pituitary-adrenal responsiveness to corticotropin-releasing hormone in patients receiving chronic, alternate day glucocorticoid therapy. J Clin Endocrinol Metab 61:22, 1985.

205. Moell C, Aronson AS, Selvik G: Growth in rabbits during alternate-day cortisone injections: near normal growth on days without cortisone. Acta Paediatr Scand 77:693-698, 1988.

206. Fauci AS: Corticosteroids in autoimmune disease. Hosp Pract pp. 99-114; October 1983.

207. Kaplan PW, Rocha W, Sanders DB, et al.: Acute steroid-induced tetraplegia following status asthmaticus. Pediatrics 78:121-123, 1986.

208. Nashel DJ: Is atherosclerosis a complication of long-term corticosteroid treatment? Am J Med 80:925-929, 1986.

209. Baer PA, Shore A, Ikeman RL: Transient fall in serum salicylate levels following intraarticular injection of steroid in patients with rheumatoid arthritis. Arthritis Rheum 30:345-347, 1987.

210. Klinenberg JR, Miller R: Effect of corticosteroids on blood salicylate concentrations. JAMA 194:601-604, 1965.

211. Tanchyk AP. Prevention of tooth erosion from salicylate therapy in juvenile rheumatoid arthritis. Gen Dent 34:479-480, 1986.

212. Forsyth BW, Horwitz RI, Acampora D, et al.: New epidemologic evidence confirming that bias does not explain the aspirin/Reye's Syndrome association. JAMA 261:2517-2524, 1989.

213. Remington PL, Shabino CL, McGee H, et al.: Reye syndrome and juvenile rheumatoid arthritis in Michigan. Am J Dis Child 139:870-872, 1985. 
214. Rennebohm RM, Heubi JE, Daughtery CC, et al.: Reye syndrome in children receiving salicylate therapy for connective tissue disease. J Pediatr 107:877-880, 1985.

215. Hollister JR: Aspirin in juvenile rheumatoid arthritis. Am J Dis Child 139:866$867,1985$.

216. Kaneshiro MM, Mielke CH Jr, Kasper CK, et al.: Bleeding time after aspirin in disorders of intrinsic clothing. N Engl J Med 281:1039-1042, 1969.

217. Quick AJ: Salicylates and bleeding: the aspirin tolerance test. Am J Med Sci 252:265-269, 1966.

218. Rajah SM, Penny A, Kester R: Aspirin and bleeding time. Lancet 2:1104, 1978.

219. Estes D, Kaplan K: Lack of platelet effect with aspirin analog salsalate. Arthritis Rheum 23:1303-1307, 1980.

220. Goldsweig HG, Kapusta M, Schwartz J: Bleeding, salicylates and prolonged prothrombin time: three case reports and a review of the literature. $\mathrm{J}$ Rheum 3:37-42, 1976.

221. Mills DG, Borda IT, Philp RB, et al.: Effects of in viro aspirin on blood platelets of gastrointestinal bleeders. Clin Pharmacol Ther 15:187-192, 1974.

222. Rees WD, Turnberg LA: Reappraisal of the effects of aspirin on the stomach. Lancet 2:410-413, 1980.

223. Manso C, Taranta A, Nydick I. Effects of aspirin administration on serum glutamic oxaloacetic and glutamic pyruvic transaminases in children. Proc Soc Exp Biol Med 93:83-88, 1956.

224. Schaller JG: Chronic salicylate administration in juvenile rheumatoid arthritis: aspirin "hepatitis" and its clinical significance. Pediatrics 62:916-925, 1978.

225. Iancu T, Elian E. Ultrastructural changes in aspirin hepatotoxicity. Am J Clin Pathol 66:570-575, 1976.

226. Levinson JE, Brewer E Jr, Hanson V, et al.: Comparison of tolmetin sodium and aspirin in the treatment of juvenile rheumatoid arthritis. J Pediatr 91:799804, 1977.

227. Leonards JR, Levy G: Aspirin-induced occult gastrointestinal blood loss: Local versus systemic effects. J Pharm Sci 59:1511-1513, 1970.

227a.Cryer B, Feldman M: Effects of non-steroidal anti-inflammatory drugs on endogenous gastrointestinal prostaglandins and therapeutic strategies for prevention and treatment of non-steroidal anti-inflammatory drug-induced damage. Arch Intern Med 152:1145-1155, 1992.

228. Hopkinson N, Doherty M: NSAID-associated gastropathy-a role for misoprostol? Br J Rheumatol 29:133-136, 1990.

229. Gonwa TA, Hamilton RW, Buckalew VM Jr: Ghronic renal failure and endstage renal disease in northwest North Carolina. Arch Intern Med 141:462-465, 1981.

230. Dixon A, Martin BK, Smith MJ, et al. (eds.): Salicylates, An International Symposium. Churchill Livingstone, London, 1963.

231. Hoftiezer JW, Burks M, Silvoso GR, et al.: Comparison of the effects of regular and enteric-coated aspirin on the gastroduodenal mucosa of man. Lancet 2:609-612, 1980.

232. Orozco-Alcala JJ, Baum J: Regular and enteric-coated aspirin: A re-evaluation. Arthritis Rheum 22:1034-1037, 1979.

233. Baum J: Aspirin in the treatment of juvenile arthritis. Am J Med 74(6A):10-15, 1983.

234. Clark RL, Lasagno L: How reliable are enteric-coated aspirin preparations? 
Clin Pharmacol Ther 6:568-574, 1965.

235. McCarty DJ, Csuka M-E: Aspirin in the treatment of chronic inflammatory arthritis. JAMA 257:1331, 1987.

236. Wiseman EH: Plasma salicylate concentrations following chronic administration of aspirin as conventional and sustained release tablets. Curr Ther Res 11:681-688, 1969.

237. American Academy of Pediatrics: Committee on School Health: Administration of medication in school. Pediatrics 74:433, 1984.

238. Wasner C, Britton MC, Kraines RG, et al.: Non-steroidal antiinflammatory agents in rheumatoid arthritis and ankylosing spondylitis. JAMA 246:21682172, 1981.

238a. McConnell RJ: Abnormal thyroid function test results in patients taking salsalate. JAMA 267:1242-1243, 1992.

239. Guindo J, Rodriguez de la Serna A, Ramio J, et al.: Recurrent PericarditisRelief with colchicine. Cirulation 82:1117-1120, 1990 (See also: Correspondence pp. 1458-1459 and 1830-1831).

240. Goldenbery J, Pessoa AP, Roizenblatt S, et al.: Cardiac tamponade in juvenile chronic arthritis: report of two cases and review of publications. Ann Rheum Dis 49:549-553, 1990.

241. Scharf J, Levy J, Benderly A, et al.: Pericardial tamponade in juvenile rheumatoid arthritis. Arthritis Rheum 19:760-762, 1976.

242. Oakes DD, Light JA, Weidig JC, et al.: Methyl prednisone in uremic pericarditis. Lancet 1:1312-1313, 1977.

243. Buselmeier TJ, Davin TD, Simmons RL, et al.: Treatment of intractable uremic pericardial effusion: avoidance of pericardiectomy with local steroid instillation. JAMA 240:1358-1359, 1978.

244. Feigenbaum SL, Masi AT, Kaplan SB: Prognosis in rheumatoid arthritis. A longitudinal study of newly diagnosed younger adult patients. Am J Med 66:377-384, 1979.

245. Maldonado-Cocco JA, Garica-Morteo O, Spindler AJ, et al.: Carpal ankylosis in juvenile rheumatoid arthritis. Arthritis Rheum 23:1251-1255, 1980.

246. Scott PJ, Ansell BM, Huskisson EG: Measurement of pain in juvenile chronic polyarthritis. Ann Rheum Dis 36:186-187, 1977.

247. Hochberg MC, Chang RW, Dwosh I, et al.: The American College of Rheumatology revised criteria for the classification of global functional status in rheumatoid arthritis. Arthritis Rheum 35:498-502, 1992.

248. Wolfe F, Hawley DJ: Remission in rhuematoid arthritis. J Rheumatol 12:245$252,1985$.

249. Nepom BS, Nepom GT, Mickleson E, et al.: Specific HLA-DR4 associated histo-compatibility molecules characterize patients with seropositive juvenile rheumatoid arthritis. J Clin Invest 76:287-291, 1984.

250. Walker SM, McCurdy DK, Shaham B, et al.: High prevalence of IgA rheumatoid factor in severe polyarticular-onset juvenile rheumatoid arthritis, but not in systemic-onset or pauciarticular-onset disease. Arthritis Rheum 33:199-204, 1990.

251. Harris ED Jr.: Rheumatoid arthritis. Pathophysiology and implications for therapy. N Engl J Med 322:1277-1289, 1990.

252. Sherry DD, Bohnsack J, Salmonson K, et al.: Painless juvenile rheumatoid arthritis. J Pediatr 116:921-923, 1990.

253. Bywaters EGL, Glynn LE, Zeldis A: Subcutaneous nodules of Still's disease. 
Ann Rheum Dis 17:278-285, 1958.

254. Hough, AJ Jr, Sokoloff L: The pathology of rheumatoid arthritis and related disorders. In McCarthy DJ (ed.) Arthritis and Allied Conditions, 11 th ed. Lea and Febiger, Philadelphia, 1989, pp. 674-697.

255. Gardiner DL: Structure and function of connective tissue and joints. In Scott JT (ed.) Copeman's Textbook of the Rheumatic Diseases, 5th ed. Churchill Livingstone, Edinburgh, 1978, pp. 78-124.

256. Scientific basis for the study of the rheumatic diseases. In McCarty DJ (ed.) Arthritis and Allied Conditions, 11th ed., Section II. Lea and Febiger, Philadelphia, 1989, pp. 189-504.

257. Stabrun AE, Larheim TA, Hoyeraal HM, Rosler M: Reduced mandibular dimensions and asymmetry in juvenile rheumatoid arthritis. Arthritis Rheum 31:602-611, 1988.

258. Engel MB, Richmond J, Brodie AG: Mandibular growth disturbance in rheumatoid arthritis in childhood. Am J Dis Child 78:728-743, 1949.

259. Myall RWT, West RA, Horwitz H, Schaller JG: Jaw deformity caused by juvenile rheumatoid arthritis and its correction. Arthritis Rheum 31:1305-1310, 1988.

260. Espada G, Babini JC, Maldonado-Cocco JA, Garcia-Morteo O: Radiologic review: the cervical spine in juvenile rheumatoid arthritis. Semin Arthritis Rhuem 17:185-195, 1988.

261. Fried JA, Athreya B, Gregg JR, et al.: The cervical spine in juvenile rheumatoid arthritis. Clin Orthop 179:102-106, 1983.

262. Espada G, Babini JC, Maldonado-Cocco JA, Garcia-Morteo O: Radiologic review: The cervical spine in Juvenile rheumatoid arthritis. Semin Arthritis Rheum 17:185-195, 1988.

263. Arroyo IL, Barron KS, Brewer EJ Jr.: Spinal cord compression by epidural lipomatosis in juvenile rheumatoid arthritis. Arthritis Rheum 31:447-451, 1988.

264. D'Arch EJ, Fell RH: Anesthesia in juvenile chronic polyarthritis. In Arden GP, Ansell BM (eds.) Surgical Management of Juvenile Chronic Polyarthritis. Academic Press. London, 1978, pp. 63-74.

265. Scott RD, Sarokhan AJ, Dalziel R: Total hip and total knee arthroplasty in juvenile rheumatoid arthritis. Clin Orthop 182:90-97, 1984.

266. Goldberg J, Kovarsky J: Beclomethasone dipropionate inhalation treatment for chronic hoarseness in rheumatic disease. Arthritis Rheum 26:1412, 1983.

267. Pearson ME, Kosco M, Huffer W, et al.: Rheumatoid nodules of the spine: case report and review of the literature. Arthritis Rheum 30:709-713, 1987.

268. Arden GP, Ansell BM (eds.). Surgical Management of Juvenile Chronic Polyarthritis. Academic Press, London, 1978.

269. Ansell BM: Juvenile arthritis. Clin Rheum Dis 10:657-672, 1984.

270. Vostrejs M, Hollister R: Muscle atrophy and leg length discrepancies in pauciarticular juvenile rheumatoid arthritis. Am J Dis Child 142:343-345, 1988.

271. Stiehm ER: Nonsteroidal anti-inflammatory drugs in pediatric patients. Am J Dis Child 142:1281-1282, 1988.

272. Rosenberg AM: Advanced drug therapy for juvenile rheumatoid arthritis. J Pediatr 114:171-178, 1989.

273. Huskisson EC: Routine drug treatment of rheumatoid arthritis and other rheumatic diseases. Clin Rheum Dis 5:697-706, 1979.

274. Herman JH, Appel AM, Hess EV: Modulation of cartilage destruction by select nonsteroidal antiinflammatory drugs. In vitro effect on the synthesis and activ- 
ity of catabolism-inducing cytokines produced by osteoarthritic and rheumatoid synovial tissue. Arthritis Rheum 30:257-265, 1987.

275. Jacobs JC, Berdon WE, Johnston AD: HLA-B27 assoicated spondyloarthritis in childhood: Clinical, pathologic and radiographic observations in 58 patients. J Pediatr 100:521-528, 1982.

276. Hart FD: Ankylosing spondylitis and related disorders. In Hart FD (ed.) Drug Treatment of the Rheumatic Diseases. Williams and Wilkins, Sydney, 1989, pp. $156-161$.

277. Luggen ME, Gartside PS, Hess EV: Nonsteroidal antiinflammatory drugs in rheumatoid arthritis: duration of use as a measure of relative value. J Rheumatol 16:1565-1569, 1989.

278. Pippenger CE: Therapeutic drug monitoring: An overview. Therap Drug Monit 1:3-9, 1979.

279. Lovell DJ, Giannini EH, Brewer EJ Jr: Time course of response to non-steroidal antiinflammatory drugs in juvenile rheumatoid arthritis. Arthritis Rheum 27:1433-1437, 1984.

280. Ehrlich GE: Tolmetin sodium: meeting the clinical challenge. Clin Rheum Dis 5:481-497, 1979.

281. Makela AL: Naproxen in the treatment of juvenile rheumatoid arthritis. Scand J Rheumatol 6:193-205, 1977.

282. Ansell BM, Hanna B, Moran H, et al.: Naproxen in juvenile chronic polyarthritis. Eur J Rheum Inflam 2:79-83, 1979.

283. Nicholls A, Hazleman B, Todd RM, et al.: Long-term evaluation of naproxen suspension in juvenile chronic arthritis. Curr Med Res Opin 8:204-207, 1982.

284. Williams PL, Ansell BM, Bell A, et al.: Multicentre study of piroxicam versus naproxen in juvenile chronic arthritis, with special reference to problem areas in clinical trials of nonsteroidal anti-inflammatory drugs in childhood. $\mathrm{Br} \mathrm{J}$ Rheumatol 25:67-71, 1986.

285. Leak AM, Richter MR, Clemens LE, et al.: A crossover study of naproxen, diclofenac and tolmetin in seronegative juvenile chronic arthritis. Clin Exp Rheumatol 6:157-160, 1988.

286. Suarez SM, Cohen PR, DeLeo VA: Bullous photosensitivity to naproxen: "pseudoporhyria". Arthritis Rheum 33:903-908, 1990.

287. Levy ML, Barron KS, Eichenfield A, Honig PJ: Naproxen-induced pseudoporphyria: a distinctive photodermatitis. J Pediatr 117:660-664, 1990.

287a.Goodwin SD, Glenny RW: Nonsteroidal anti-inflammatory drug-associated infiltrates with eosinophilia. Arch Intern Med 152:1521-1524, 1992.

288. Giannini EH, Brewer EJ, Miller ML, et al.: Ibuprofen suspension in the treatment of juvenile rheumatoid arthritis. J Pediatr 117:645-652, 1990.

289. Rhymer AR, Gengos DC: Indomethacin. Clin Rheum Dis 5:541-552, 1979.

290. Jacobs JC: Sudden death in arthritic children receiving large doses of indomethacin. JAMA 199:932-934, 1967.

291. Editorial. Analgesics, agranulocytosis, and aplastic anaemia: A major casecontrolled study. Lancet 2:899-900, 1986.

292. Brewer EJ, Giannini EH, Baum J, et al.: Aspirin and fenoprofen (Nalfon ${ }^{\mathrm{R}}$ ) in the treatment of juvenile rheumatoid arthritis: results of the double blind-trial. A Segment II Study. J Rheumatol 9:123-128, 1982.

293. Bass J, Athreya B, Brandstrup N, et al.: Flurbiprofen in the treatment of juvenile rheumatoid arthritis. J Rheumatol 13:1081-1083, 1986.

294. Brewer EJ, Giannini EH, Baum J, et al.: Ketoprofen $\left(\right.$ Orudis $\left.^{\mathrm{R}}\right)$ in the treatment 
of juvenile rheumatoid arthritis. A Segment I Study. J Rheumatol 9:144-148, 1982.

295. Brewer EJ, Giannini EH, Baum J, et al.: Proquazone $\left(\right.$ Biarsan $\left.^{\mathrm{R}}\right)$ in the treatment of juvenile rheumatoid arthritis: A segment I study. J Rheumatol 5:135139, 1982.

296. Brewer EJ, Giannini EH, Baum J, et al.: Sodium meclofenamate (Meclomen ${ }^{\mathrm{R}}$ ) in the treatment of juvenile rheumatoid arthritis. A segment I Study. J Rheumatol 9:129-134, 1982.

297. Bass JC, Giannini EH, Brewer EJ, et al.: Pirprofen $\left(\right.$ Rengasil $\left.{ }^{\mathrm{R}}\right)$ in the treatment of juvenile rheumatoid arthritis. A segment I study. J Rheumatol 9:140-143, 1982.

298. Bhettay E: Double-blind study of sulindac and aspirin in juvenile chronic arthritis. SAMJ 70:724-726, 1986.

299. Haapasaari J, Wuolijoki E, Ylijoki H: Treatment of juvenile rheumatoid arthritis with diclofenac sodium. Scand J Rheumatol 12:325-330, 1983.

300. Helfgott SM, Sandberg-Cook J, Zakim D, Nestler J: Diclofenac-associated hepatotoxicity. JAMA 264:2660-2662 (see also editiorial pp. 2677-2678).

301. Paulus HE: FDA Arthritis Advisory Committee Meeting: risks of agranulocytosis/aplastic anemia, flank pain, and adverse gastrointestinal effects with use of nonsteroidal antiinflammatory drugs. Arthritis Rheum 30:593-595, 1987.

302. Cush JJ, Jasin HE, Johnson R, Lipsky PE: Relationship between clinical efficacy and laboratory correlates of inflammatory and immunologic activity in rheumatoid arthritis patients treated with nonsteroidal antiinflammatory drugs. Arthritis Rheum 33:623-633, 1990.

303. Caille G, du Souich R, Gervais $P$, et al.: Effects of concurrent sucralfate administration on pharmacokinetics of naproxen. Am J Med 83 (Suppl 38) 67-73, 1987.

304. Howes CA, Pullar T, Sourindhrin I, et al.: Reduced steady-state plasma concentrations of chlorpromazine and indomethacin in patients receiving cimetidine. Eur J Clin Pharmacol 24:99-102, 1983.

305. Graham DY: Prevention of gastroduodenal injury induced by chronic nonsteroidal antiinflammatory drug therapy. Gastroenterology 96:675-681, 1989.

306. Roth S, Agrawal N, Mahowald M, et al.: Misoprostol heals gastroduodenal injury in patients with rheumatoid arthitis receiving aspirin. Arch intern Med 149:775-779, 1989.

307. Furst DE, Sarkissian E, Blocka K, et al.: Serum concentrations of salicylate and naproxen during concurrent therapy in patients with rheumatoid arthritis. Arthritis Rheum 30:1157-1161, 1987.

307a.O'Brien WM, Bagby GF: Rare adverse reactions to nonsteroidal antiinflammatory drugs. J Rheumatol 12:13-20, 347-353, 562-567, 785-789, 1985.

307b.Hyson CP, Kazakoff MA: A servere multisystem reaction to sulindac. Arch Intern Med 151:387-388, 1991.

308. Barrier $\mathrm{CH}$, Hirschowitz BI: Controversies in the detection and management of nonsteroidal antiinflammatory drug-induced side effects of the upper gastrointestinal tract. Arthritis Rheum 32:926-932, 1989.

309. Langman MJS: Epidemiologic evidence on the association between peptic ulceration and antiinflammatory drug use. Gastroenterology 96:640-646, 1989.

310. Fries JF, Miller SR, Spitz PW, et al.: Toward an epidemiology of gastropathy associated with nonsteroidal antiinflammatory drug use. Gastroenterology 96:647-655, 1989. 
310a.Gabriel SE, Bombardier C: NSAID induced ulcers. An emerging epidemic? J Rheumatol 17:1-4, 1990.

310b.Gibson GR, Whitacre EB, Ricotti CA: Colitis induced by nonsteroidal antiinflammatory drugs. Report of four cases and review of the literature. Arch Intern Med 152:625-632, 1992.

311. Hoppmann RA, Peden JG, Ober SK: Central nervous system side effects of nonsteroidal anti-inflammatory drugs. Aseptic meningitis, psychosis, and cognitive dysfunction. Arch Intern Med 151:1309-1013, 1991.

312. Lindsley CB, Warady BA: Nonsteroidal antiinflammatory drugs: renal toxicity: review of pediatric issues. Clin Pediatr 29:10-113, 1990.

313. Robinson J, Malleson P, Lirenman D, Carter J: Nephrotic syndrome associated with nonsteroidal anti-inflammatory drug use in two children. Pediatrics 85:844-847, 1990.

314. Tietjan DP: Recurrence and specificity of nephrotic syndrome due to tolmetin. Am J Med 87:354-355, 1989.

315. Allen RC, Petty RE, Lirenman DS, et al.: Renal papillary necrosis in children with chronic arthritis. Am J Dis Child 140:20-22, 1986 (see also editorial pp. 16-17).

316. Laxer RM, Silverman ED, Balfe J-W, et al.: Naproxen-associated renal failure in a child with arthritis and inflammatory bowel disease. Pediatrics 80:904-908, 1987.

317. Dunn MJ, Patrono C (eds.): Renal effects of nonsteroidal anti-inflammatory drugs. AJM 81(2B):1-132, 1986.

318. Warren GV, Korbet SM, Schwartz MM, Lewis EJ: Minimal change glomerulopathy associated with nonsteroidal antiinflammatory Drugs. Am J Kidney Dis 13:127-130, 1989.

319. Schwarz A, Krause PH, Keller F, et al.: Granulomatous interstitial nephritis after nonsteroidal anti-inflammatory Drugs. Am J Nephrol 8:410-416, 1988.

320. Stillman MT, Schlesinger PA: Nonsteroidal anti-inflammatory drug nephrotoxicity. Should we be concerned? Arch Intern Med 150:268-270, 1990.

321. Ten RM, Torres VE, Milliner DS, et al.: Acute interstitial nephritis: Immunologic and clinical aspects. Mayo Clin Proc 63:921-930, 1988.

322. Garella S, Matarese RA: Renal effects of prostaglandins and clinical adverse effects of nonsteroidal anti-inflammatory agents. Medicine 63:165-181, 1984.

323. Bender WL, WHelton A, Beschorner WE, et al.: Interstitial nephritis, proteinuria, and renal failure caused by nonsteroidal anti-inflammatory drugs. Immunologic characterization of the inflammatory infiltrate. Am J Med 76:10061012, 1984.

324. Clive DM, Stoff JS: Renal syndromes associated with nonsteroidal antiinflammatory drugs. N Eng J Med 310:563-572, 1984.

324a.Cronberg S, Wallmark E, Soderberg I: Effect on platelet aggregation of oral administration of 10 non-steroidal analgesics to humans. Scand J Haematol 33:155-159, 1984.

324b.Connelly GS, Panush RS: Should nonsteroidal anti-inflammatory drugs be stopped before elective surgery? Arch Intern Med 151:1963-1966, 1991.

325. Szer IS, Goldenstein-Schainberg C, Kurtin PS: Paucity of renal complications associated with nonsteroidal antiinflammatory drugs in children with chronic arthritis. J Pediatr 119:815-817, 1991.

326. Iannuzzi L, Dawson N, Zein N, Kushner I: Does drug therapy slow radiologic deterioration in rheumatoid arthritis? N Eng J Med 309:1023-1028, 1983. 
327. Scott DL, Symmons DPM, Coulton BL, Popert AJ: Long-term outcome of treating rheumatoid arthritis: results after 20 years. Lancet 1:1108-1111, 1987.

328. Pincus $\mathrm{T}$ (ed.) Rheumatoid arthritis: disappointing long-term outcomes despite successful short-term clinical trials. J Clin Epidemiol 41:1037-1041, 1988.

329. Dawes PT, Powler S, Clarke J, et al.: Rheumatoid arthritis: treatment which controls the C-reactive protein and erythrocyte sedimentation rate reduces radiological progression. Br J Rheumatol 25:44-49, 1986.

330. Grondin C, Malleson P, Petty RE: Slow-acting antirheumatic drugs in chronic arthritis of childhood. Semin Arthritis Rheum 18:38-47, 1988.

331. Giannini EH, Brewer EJ, Kuzmina N, et al.: Characteristics of responders and nonresponders to slow-acting antirheumatic drugs in juvenile rheumatoid arthritis. Arthritis Rheum 31:15-20, 1988.

332. Manners PJ, Ansell BM: Slow-acting antirheumatic drug use in systemic onset juvenile chronic arthritis. Pediatrics 77:99-103, 1986.

333. Rosenberg AM: Advanced drug therapy for juvenile rheumatoid arthritis. J Pediatr 114:171-178, 1989.

334. van der Heijde DM, van Riel PL, Nuver-Zwart IH, et al.: Effects of hydroxychloroquine and sulphasalazine on progression of joint damage in rheumatoid arthritis. Lancet 1:1036-1038, 1989.

335. Williams HJ, Ward JR, Dahl SL, et al.: A controlled trial comparing sulfasalazine, gold sodium thiomalate, and placebo in rheumatoid arthritis. Arthritis Rheum 31:702-713, 1988.

336. Ozdogan H, Turunc M, Deringol B, et al.: Sulphasalazine in the treatment of juvenile rheumatoid arthritis: a preliminary open trial. J Rheumatol 13:124$125,1986$.

337. Jacobs JC: Sulphasalazine in the treatment of childhood arthritis: a preliminary report. Arthritis Rheum 33:S145, 1990.

338. Pinals RS: Sulfasalazine in the rheumatic disease. Semin Arthritis Rheum 17:246-259, 1988.

339. Farr M, Kitas G, Waterhouse L, et al.: Sulfasalazine in psoriatic arthritis-A double blind placebo-controlled study. Br J Rheumatol 29:46-49, 1990.

340. Job-Deslandre C, Menkes C-J. Sulphasalazine in the treatment of juvenile spondylarthropathy. Arthritis Rheum 34:S153, 1991.

341. Ansell BM, Hall MA, Woo LP, et al.: A multicentre pilot study of sulphasalazine in juvenile chronic arthritis. Clin Exp Rheumatol 9:201-203, 1991.

342. Ansell BM: Juvenile chronic arthritis and juvenile spondyloarthropathy. Gurrent Opin Rheumatol 3:838-843, 1991.

343. Hertzberger-Ten Cate R, Cats A: Toxicity of sulfasalazine in systemic juvenile chronic arthritis. Clin Exp Rheumatol 9:85-88, 1991.

344. Sherry DD, Wallace CA. Sulfasalazine toxicity in children with systemic onset juvenile rheumatoid arthritis. Arthritis Rheum 34:S153, 1991.

345. Felson DT, Andserson JJ, Meenan RF: The comparative efficacy and toxicity of second-line drugs in rheumatoid arthritis. Results of two metaanalyses. Arthritis Rheum 33:1449-1461, 1990.

346. Taffet SL, Das KM: Sulfasalazine: Adverse effects and desensitization. Dig Dis Sci 28:833-842, 1983.

347. Amos RS, Pullar T, Bax DE, et al.: Sulphasalazine for rheumatoid arthritis: toxicity in 774 patients monitored for one to 11 years. Br Med J 293:420-423, 1986.

348. Marabani M, Madhok R, Capell HA, Hunter JA: Leuocopenia during sulpha- 
salazine treatment for rheumatoid arthritis. Ann Rheum Dis 48:505-507, 1989.

349. Farr M, Tunn EJ, Symmons DPM, et al.: Sulphasalazine in rheumatoid arthritis: haematological problems and changes in haematological indices associated with therapy. Br J Rheumatol 28:134-138, 1989.

350. Valcke Y, Pauwels R, van der Straeten M: Bronchoalveolar lavage in acute hypersensitivity pneumonitis caused by sulfasalazine. Chest 92:572-573, 1987.

351. Deboever G, Devogelaere R, Holvoet G: Sulphasalazine-induced lupus-like syndrome with cardiac tamponada in a patient with ulcerative colitis. Am J Gastroenterol 84:85-86, January 1989.

352. Epstein WV: Parental gold therapy for rheumatoid arthritis: A treatment whose time has gone. J Rheumatol 16:1291-1294, 1989.

353. Jessop, JD: Gold in the treatment of rheumatoid arthritis-why, when, how? J Rheum 6(Suppl. 5):12-17, 1979.

354. Empire Rheumatism Council: Gold therapy in rheumatoid arthritis. Final report of a multicenter controlled trial. Ann Rheum Dis 20:315-354, 1961.

355. Epstein WV, Henke CJ, Yelin EH, Katz PP: Effect of parenterally administered gold therapy on the course of adult rheumatoid arthritis. Ann Intern Med 114:437-444, 1991.

356. Levinson JE, Balz GP, Bondi S: Gold therapy. Arthritis Rheum 20:531-535, 1977.

357. Hall MA, Ansell BM, Spencer PE: A comparative study of gold and penicillamine in juvenile chronic arthritis. Presented at the 15th International Congress of Rheumatology, Paris, 1981. Rev Rhum 48(Suppl):, abstract 1290, 1981.

358. Davis P: Undersirable effects of gold salts. J Rheum 6(Suppl. 5):18-24, 1979.

358a.Hansen RM, Varma RR, Hanson, GA: Gold induced hepatitis and pure red cell aplasia. Complete recovery after corticosteroid and N-Acetylcysteinc therapy. J Rheumatol 18:1251-1253, 1991.

359. Wooley PH, Criffin J, Panayi GS, et al.: HLA-DR antigens and toxic reaction to sodium aurothiomalate and $\mathrm{D}$-penicillamine in patients with rheumatoid arthritis. N Engl Med 303:300-302, 1980.

360. Takehara $M$, Beardmore $T$ : Self administration of parenteral gold and methotrexate in rheumatoid arthritis. Arthritis Rheum 33:S16, 1990.

361. Gottlieb NL: Metabolism and distribution of gold compounds. J Rheum 6(Suppl. 5):2-6, 1979.

362. Gottlieb NL: Gold excretion and retention during auranofin treatment: A preliminary report. J Rheum 6(Suppl. 5):61-67, 1979.

363. Kvien TK, Hoyeraal HM, Sandstad B: Gold sodium thiomalate and Dpenicillamine. A controlled, comparative study in patients with pauciarticular and polyarticular juvenile rheumatoid arthritis. Scand J Rheumatol 14:346$354,1985$.

364. Giannini EH, Brewer EJ Jr, Kuzmina N, et al.: Auranofin in the treatment of juvenile rheumatoid arthritis. Results of the USA-USSR double-blind, placebocontrolled trial. Arthritis Rheum 33:466-476, 1990 (see also correspondence 34:934-935, 1991).

365. Katz WA, Blodgett RC Jr., Pietrusko RG: Proteinuria in gold-treated rheumatoid arthritis. Ann Intern Med 101:176-179, 1984.

366. Marcolongo R, Mathieu A, Pala R, et al.: The efficacy and safety of auranofin in the treatment of juvenile rheumatoid arthritis. Arthritis Rheum 31:979-983, 1988.

367. Giannini EH, Brewer EJ, Person DA: Blood gold concentrations in children 
with juvenile rheumatoid arthritis undergoing long-term oral gold therapy. Ann Rheum Dis 43:228-231, 1984.

368. Giannini EH, Barron KS, Spencer CH, et al.: Auranofin therapy in juvenile rheumatoid arthritis: results of the five-year open-label extension trial, J Rheumatol 18:1240-1242, 1991.

369. Bombardier C, Ware J, Russell IJ, et al.: Auranofin therapy and quality of life in patients with rheumatoid arthritis. Am J Med 81:565-578, 1986.

370. Jaffe IA: Penicillamine treatment of rheumatoid arthritis-rationale, pattern of clinical response, and clinical pharmacology and toxicology. In Munthe E (ed.) Penicillamine Research Rheumatoid Disease (Penicillamine Symposium). Fabritius, Oslo, Norway, 1976. pp. 11-24.

371. Multz CV: Cholestatic hepatitis caused by penicillamine. JAMA 246:674-675, 1981.

372. Hamilton EDB, Dixon A St J, Davis J, et al.: Multicentre trial with synthetic D-penicillamine in rheumatoid arthritis. JAMA 246:215-218, 1981.

373. Hill HFH: Treatment of rheumatoid arthritis with penicillamine. Semin Arthritis Rheum 6:361-388, 1977.

374. Kean WF, Dwosh IL, Anastassiades TP, et al.: The toxicity pattern of penicillamine therapy. A guide to its use in rheumatoid arthritis. Arthritis Rheum 23:158-164, 1980.

375. Schairer H, Stoeber E: Long-term follow-up of 235 cases of juvenile rheumatoid arthritis treated with D-penicillamine. In Munthe E (ed.) Penicillamine Research in Rheumatoid Disease (Penicillamine Symposium). Fabritius, Oslo, Norway, 1977, pp. 279-281.

376. Ansell BW, Hall MA: Penicillamine in chronic arthritis in childhood. J. Rheum 8(Suppl. 7):112-115, 1981.

377. Brewer EJ, Giannini EH, Kuzmina N, Alekseev L: Penicillamine and hydroxychloroquine in the treatment of severe juvenile rheumatoid arthritis. Results of the U.S.A.-U.S.S.R. double-blind placebo-controlled trial. New Eng J Med 314:1269-1276, 1986 (see also editorial pp. 1312-1314).

378. Prieur AM, Piussan C, P Manigne, et al.: Evaluation of D-penicillamine in juvenile chronic arthritis. A double-blind mutlicentre study. Arthritis Rheum 28:376-382, 1985.

379. Felson DT, Anderson JJ, Meenan RF: Time for changes in the design, analysis, and reporting of rheumatoid arthritis clinical trials. Arthritis Rheum 33:140149, 1990.

380. Paulus HE, Egger MJ, Ward JR, et al.: Analysis of improvement in individual rheumatoid arthritis patients treated with disease-modifying antirheumatic drugs, based on the findings in patients treated with placebo. Arthritis Rheum 33:477-484, 1990.

381. Hall CL, Jawad S, Harrison PR, et al.: Natural course of penicillamine nephropathy: a long term study of 33 patients. Br Med J 296:1083-1086, 1988.

382. Halla JT, Fallahi S, Koopman WJ: Penicillamine-induced myositis. Observations and unique features in two patients and review of the literature. Am J Med 77:719-722, 1984.

383. Jaffe IA: Adverse effects profile of sulfhydryl compounds in man. Am J Med 80:471-476, 1986.

384. Benson EA, Healey LA, Barron EJ: Insulin antibodies in patients receiving penicillamine. Am J Med 78:857-860, 1985. 
385. van Kerckhove C, Giannini EH, Lovell DJ: Temporal patterns of response to D-penicillamine, hydroxychloroquine and placebo in juvenile pheumatoid arthritis. Arthritis Rheum 31:1252-1258, 1988.

386. Huskisson EG: Drugs which apparently affect the rheumatoid disease process. In Hart FD (ed.) Drug Treatment of the Rheumatic Disease. Williams and Wilkins, Sydney, 1987, pp. 65-87.

387. Zvaifler NJ (ed): Update in rheumatology: focus on hydroxychloroquine. Am J Med 85:1-71, 1988.

388. Laaksonen A, Koskiahde V, Juka K: Dosage of antimalarial drugs for children with juvenile rheumatoid arthritis and systemic lupus erythematosus. A clinical study with determination of serum concentration of chloroquine and hydroxychloroquine. Scand J Rheum 3:103-108, 1974.

389. Stillman S: Antimalarials. In Moore TD (ed.) Arthritis in Childhood. Report of the 80th Ross Conference on Pediatric Research. Xanadu, Bahamas, December 2-5, 1979. Ross Laboratories. Columbus, OH, 1981, pp. 125-127.

390. Kvien TK, Hoyeraal HM, Sandstad B: Slow acting antirheumatic drugs in patients with juvenile rheumatoid arthritis-evaluated in a randomized, parallel 50-week clinical trial. J Rheumatol 12:533-539, 1985.

391. Maksymowych W, Russell AS: Antimalarials in rheumatology: efficacy and safety. Semin Arthritis Rheum 16:206-221, 1987.

392. Rynes, RI, Krohel G, Falbo A, et al.: Ophthalmologic safety of long-term hydroxychloroquine treatment. Arthritis Rheum 22:832-836, 1979.

392a.Bernstein HN: Ocular Safety of hydroxychloroquine. Ann Ophthalmol 23:292-296, 1991.

393. Beultler E: Glucose-6-phosphate dehydrogenase deficiency. N Eng J Med 324:169-174, 1991.

394. Fries JF, Singh G, Lenert L, Furst DE: Aspirin, hydroxychloroquine, and hepatic enzyme abnormalities with methotrexate in rheumatoid arthritis. Arthritis Rheum 33:1611-1619, 1990.

395. Jaffe KM, Havens PL, et al.: Childhood chloroquine poisonings-Wisconsin and Washington. JAMA 260:1361, 1988.

396. Ratliff NB, Estes ML, Myles JL, et al.: Diagnosis of chloroquine cardiomyopathy by endomyocardial biopsy. N Eng J Med 316:191-193, 1987.

397. Estes ML, Ewing-Wilson D, Chou SM, et al.: Chloroquine neuromyotoxicity: clinical and pathologic perspective. Am J Med 82:447-455, 1987.

398. Wallace CA, Bleyer WA, Sherry DD, et al.: Toxicity and serum levels of methotrexate in children with juvenile rheumatoid arthritis. Arthritis Rheum 32:677-681, 1989.

399. Shiroky JH, Watts CS, Neville C: Combination methotrexate and sulfasalazine in the management of rheumatoid arthritis: case observations. Arthritis Rheum 32:1160-1164, 1989.

400. Klippel JH, Strober S, Wofsy D: New therapies for the rheumatic diseases. Bull Rheum Dis 38(\#4):1-8, 1989.

401. Paulus HE: The use of combinations of disease-modifying antirheumatic agents in rheumatoid arthritis. Arthritis Rheum 33:113-120, 1990.

401a.Williams HJ, Ward JR, Reading JC, et al.: Comparison of auranofin, methotrexate, and the combination of both in the treatment of rheumatoid arthritis. A controlled clinical trial. Arthritis Rheum 35:259-269, 1992.

402. Arthritis Foundation: Challenging the pyramid. A new look at therapeutic 
approaches for rheumatoid arthritis. J Rheumatol 17:Suppl 25, Nov. 1990. (See also Editorial, 17:1115-1118, 1990.)

402a.McCarty DJ: Suppress rheumatoid inflammation early and leave the pyramid to the Egyptians. J Rheum 17:1115-1118, 1990.

403. Jacobs JC: Juvenile rheumatoid arthritis. In Downey JA, Low NL (eds.) The Child with Disabling Illness, 2nd ed. Raven Press, New York, 1982, pp. 3-27.

404. Joint Committee of the Medical Research Council and Nuffield Foundation: a comparison of prednisolone with aspirin or other analgesics in the treatment of rheumatoid arthritis. Ann Rheum Dis 18:173, 1959.

405. Harris ED Jr, Emkey RD, Nichols JE, Newberg A: Low dose prednisone therapy in rheumatoid arthritis: a double blind study. J Rheumatol 10:713-721, 1983.

406. Jacobs JC: The arthritic child and the family: Staying in the mainstream. Arthritis Rheum 20:595-597, 1977.

407. De Palma AF, McKeeve CD, Jubin DK: Process of repair of articular cartilage demonstrated by histology and autoradiography with tritiated thymidine. Clin Orthop 48:229-242, 1966.

408. Palmoski M, Perricone E, Brandt KD: Development and reversal of a proteoglycan aggregation defect in normal canine knee cartilage after immobilization. Arthritis Rheum 22:508-517, 1979.

409. Palmoski MJ, Colyer RA, Brandt TKD: Joint motion in the absence of normal loading does not maintain normal articular cartilage. Arthritis Rheum 23:325$334,1980$.

410. Johnson RF: The effect of immobilization on ligaments and ligamentous healing. Contemp Orthop 2:237-241, 1980.

411. Bernstein B, Forrester D, Singsen B, et al.: Hip joint restoration in juvenile rheumatoid arthritis. Arthritis Rheum 20:1099-1104, 1977.

412. Elsasser U, Wilkins B, Hesp R: Bone rarefaction and crush fractures in juvenile chronic arthritis. Arch Dis Child 57:377-380, 1982.

413. Jacobs JC, Dick HM, Downey JA, et al.: Weight bearing as a treatment for damaged hips in juvenile rheumatoid arthritis. N Engl J Med 305:409, 1981.

414. McAllister WAC, Thompson RJ, Al-Habet SM, et al.: Rifampin reduces effectiveness and bioavailability of prednisolone. Br Med J 286:923-925, 1983.

415. Nishioka $T$, Kurayama $H$, Yasuda $T$, et al.: Nasal administration of salmon calcitonin for prevention of glucocorticord-induced osteosporosis in children with nephrosis. J Pediatr 118:703-707, 1991.

416. Loftus J, Allen R, Hesp R, et al.: Randomized, double-blind trial of Deflazacort versus prednisone in juvenile chronic (or rheumatoid) arthritis: A relatively bone-sparing effect of Deflazacort. Pediatrics 88:428-436, 1991.

417. Liebing MR, Leib E, MeLaughlin K, et al.: Pulse methylprednisolone in rheumatoid arthritis. Ann Intern Med 94:21-26, 1981.

418. Miller JJ: Prolonged use of intravenous steroid pulses in the rheumatic diseases of children. Pediatrics 65:980-994, 1980.

419. Barry M: The use of high-dose pulse methylprednisolone in rheumatoid arthritis. Unproved therapy. Arch Intern Med 145:1483-1484, 1985.

420. Silverman ED, Laxer RM, Greenwald M, et al.: Intravenous gamma globulin therapy in systemic juvenile rheumatoid arthritis. Arthritis Rheum 33:1015$1022,1990$.

421. Groothoff JW, van Leeuwen EF: High dose intravenous gammaglobulin in 
chronic systemic juvenile arthritis. Br Med J 296:1362-1363, 1988.

422. Becker H, Mitropoulou G, Helmke K: Immunomodulating therapy of rheumatoid arthritis by high-dose intravenous immunoglobulin. Klin Wochenschr 67:286-290, 1980.

423. Combe B, Cosso B, Clot J, et al.: Human placenta-eluted gammaglobulins in immunomodulating treatment of rheumatoid arthritis. Am J Med 78:920-928, 1985.

424. Landsbury J: Quantitation of the activity of rheumatoid arthritis. I. A method for recording its systemic manifestations. Am J Med Sci 231:616-621, 1956.

425. Bunch TW, O'Duffy JD: Disease modifying drugs for progressive rheumatoid arthritis. Mayo Clin Proc 55:161-179, 1980.

426. Wallace CA, Bleyer WA, Sherry DD, et al.: Toxicity and serum levels of methotrexate in children with juvenile rheumatoid arthritis. Arthirits Rheum 32:677-681, 1989.

427. Rose CD, Singsen BH, Eichenfield AH, et al.: Safety and efficacy of methotrexate therapy for juvenile rheumatoid arthritis. J Pediatr 117:653-659, 1990. (See also: Correspondence 119:333-334, 1991.)

428. Singh G, Fries JF, Williams CA, et al.: Toxicity profiles of disease modifying antirheumatic drugs in rheumatoid arthritis. J Rheumatol 18:188-194, 1991.

429. Jeurissen ME, Boerbooms AM, van de Putte LB, et al.: Influence of methotrexate and azathioprine on radiologic progression in rheumatoid arthritis. A randomized double-blind study. Ann Intern Med 114:999-1004, 1991.

430. Kremer JM, Phelps CT: Long-term prospective study of the use of methotrexate in the treatment of rheumatoid arthritis. Update after a mean of 90 months. Arthritis Rheum 35:138-45, 1992.

431. Williams HJ, Willkens RF, Samuelson CO, et al.: Comparison of low-dose oral pulse methotrexate and placebo in the treatment of rheumatoid arthritis. A controlled clinical trial. Arthritis Rheum 28:721-730, 1985.

432. Truckenbrodt H, Hafner R: Methotrexate therapy in juvenile rheumatoid arthritis: a retrospective study. Arthritis Rheum 29:801-807, 1986.

433. Giannini EH, Brewer EJ, Kuzmina N, et al. Methotrexate in resistant juvenile rheumatoid arthritis. NEJM 326:1043-1049, 1992.

434. Graham LD, Myones BL, Rivas-Chacon RF, Pachman LM: Morbidity associated with long-term methotrexate therapy in juvenile rheumatoid arthritis. J Pediatr 120:468-73, 1992.

435. Giannini EH, Shaikov A, Maximov A, et al.: Meta-analysis of antirheumatic drug trials in juvenile rheumatoid arthritis. Arthritis Rheum 34:S152, 1991.

436. Wallace C, Sherry D, Salmonson K: Treatment of juvenile rheumatoid arthritis with higher dose methotrexate. Arthritis Rheum 33:S39, 1990.

437. Jundt JM, Browne BA, Mock D, et al.: Methotrexate pharmacokinetics in rheumatoid arthritis. Arthritis Rheum 33:S61, 1990.

438. Mandel MA: The synergistic effect of salicylates on methotrexate toxicity. Plast Recontruct Surg 57:733-737, 1976.

439. Taylor JR, Halprin KM: Effect of sodium salicylate and indomethacin on methotrexate-serum albumin binding. Arch Dermatol 113:588-591, 1977.

439a. Dupuis LL, Koren G, Shore A, et al.: Methotrexate-Nonsteroidal antiinflamatory drug interaction in children with arthritis. J Rheumatol 17:1469-1473, 1990.

440. Weinblatt ME, Weissman BN, Holdsworth DE, et al.: Long-term prospective 
study of methotrexate in the treatment of rheumatoid arthritis. 84-month update. Arthritis Rheum 35:129-37, 1992.

441. Nordstrom DM, West SG, Andersen PA, Sharp JT: Pulse methotrexate therapy in rheumatoid arthritis. Ann Intern Med 107:797-801, 1987.

441a.Rau R, Herborn G, Karger T, Werdier D: Retardation of radiologic progression in rheumatoid arthritis with methotrexate therapy. A controlled study. Arthritis Rheum 84:1236-1244, 1991.

442. Kremer JM, Rynes RI; Bartholomew LE: Severe flare of rheumatoid arthritis after discontinuation of long-term methotrexate therapy. Am J Med 82:781786, 1987.

443. Flowers MA, Heathcote J, Wanless IR, et al.: Fulminant hepatitis as a consequence of reactivation of hepatitis $B$ virus infection after discontinuation of lowdose methotrexate therapy. Ann Intern Med 112:381-382, 1990.

444. Alarcon GS, Tracy IC, Blackburn WD Jr.: Methotrexate in rheumatoid arthritis. Toxic effects as the major factor in limiting long-term treatment. Arthritis Rheum 32:671-676, 1989.

445. Bjorkman DJ, Hammond EH, Lee RG, et al.: Hepatic ultrastructure after methotrexate therapy for rheumatoid arthritis. Arthritis Rheum 31:1465-1472, 1988.

446. Aponte J, Petrelli M: Histopathologic findings in the liver of rheumatoid arthritis patients treated with long-term bolus methotrexate. Arthritis Rheum 31:1457-1464, 1988.

447. Kremer JM, Lee RG, Tolman KG: Liver histology in rheumatoid arthritis patients receiving long-term methotrexate therapy. Arthritis Rheum 32:121-127, 1989.

448. Shergy WJ, Polisson RP, Caldwell DS, et al.: Methotrexate-associated hepatotoxicity: retrospective analysis of 210 patients with rheumatoid arthritis. Am J Med 85:771-774, 1988.

449. Brick JE, Moreland LW, Al-Kawas F, et al.: Prospective anaylsis of liver biopsies before and after methotrexate therapy in rheumatoid patients. Semin Arthritis Rheum 19:31-44, 1989.

449a. Keim D, Ragadalc C, Heidelberger K, Sullivan D: Hepatic fibrosis with the use of methothexate for juvenile arthritis. J Rheumatol 17:846-848, 1990.

450. Phillips C, Cera P, Mangan T, Newman E: Severe liver disease (SLD) in rheumatoid arthritis (RA) patients on methotrexate (MTX). Arthritis Rheum 33:\#5 (Suppl):R15, 1990.

451. Whiting-O'Keefe QE, Fye KH, Sack KD: Methotrexate and histologic hepatic abnormalities: A meta-analysis. Am J Med 90:711-716, 1991.

452. Keim DR, Godoshian-Ragsdale C, Sullivan DB: Liver biopsy with the use of methotrexate for juvenile rheumatoid arthritis. J Pediatr 118:654, 1991.

452a.Hall P ole la M, Ahern MJ, Jarvis LR, et al.: Two methods of assessment of methotrexate hepatotoxicity in patients with rheumatoid arthritis. Ann Rheum Dis 50:471-476, 1991.

453. Carson CW, Cannon GW, Egger MJ, et al.: Pulmonary disease during the treatment of rheumatoid arthritis with low dose pulse methotrexate. Semin Arthritis Rheum 16:186-195, 1987.

454. Furst DE, Kremer JM: Methotrexate in rheumatoid arthritis. Arthritis Rheum 31:305-314, 1988.

455. Wernick R, Smith DL: Central nervous system toxicity associated with weekly 
low-dose methotrexate treatment. Arthritis Rheum 32:770-775, 1989.

456. Shupack JL, Webster GF: Pancytopenia following low-dose oral methotrexate therapy for psoriasis. JAMA 259:3594-3596, 1988.

457. Scalone J, Bowen JR: Methotrexate osteopathy: case report. Contemp Orthop 17:46-49, 1988.

458. Lopez-Mendez A, Bridges SL Jr., Han K, et al.: Methotrexate increases risk of post-surgical complications in rheumatoid arthritis patients. Arthritis Rheum 33:\#5 (Suppl) R131, 1990.

459. Tugwell P, Bombardier C, Buchanan WW, et al.: Methotrexate in rheumatoid arthritis. Impact on quality of life assessed by traditional standard-item and individualized patient preference health status questionnaires. Arch Intern Med 150:59-62, 1990.

460. Weinblatt ME, Kaplan H, Germain BF, et al.: Low-dose methotrexate compared with auranofin in adult rheumatoid arthritis. A thirty-six week, doubleblind trial. Arthritis Rheum 33:330-338, 1990.

461. Shiroky J, Neville C, Esdaile J, Skelton J: High dose methotrexate with leucovorin rescue in the management of rheumatoid arthritis. Arthritis Rheum 33:\#5 (Suppl), p. R39, 1990.

462. Hamdy H, McKendry RJR, Mierins E, Liver JA: Low-dose methotrexate compared with azathioprine in the treatment of rheumatoid arthritis. A twenty-fourweek controlled clinical trial. Arthritis Rheum 30:361-368, 1987.

463. Kvien TK, Hoyeraal HM, Sandstad B: Azathioprine versus placebo in patients with rheumatoid arthritis: A single center double blind comparative study. J Rheumatol 13:118-123, 1986.

464. Silman AJ, Petrie J, Hazleman B, et al.: Lymphoproliferative cancer and other malignancy in patients with rheumatoid arthritis treated with azathioprine: A 20 year follow up study. Ann Rheum Dis 47:988-992, 1988.

465. Jeurissen MEC, Boerbooms AM Th, van de Putte LBA, Kruijsen MWM: Azathioprine induced fever, chills, rash, and hepatotoxicity in rheumatoid arthritis. Ann Rheum Dis 49:25-27, 1990.

465a. Willkens RF, Urowitz MB, Stablein DM, et al.: Comparison of azathioprine, methotrexate, and the combination of both in the treatment of rheumatoid arthritis. Arthritis Rheum 35:849-856, 1992.

466. Singh G, Fries JF, Spitz P, Williams CA: Toxic effects of azathioprine in rheumatoid arthritis. Arthritis Rheum 32:837-843, 1989.

467. Hardin JG: Cytotoxic-immunosuppressive drugs in the treatment of rheumatoid arthritis. Interm Med 8(12):123-137, 1987.

468. Baker GL, Kehl LE, Zee BC, et al.: Malignancy following treatment of rheumatoid arthritis with cyclophosphamide. Long-term case-control follow-up study. Am J Med 83:1-9, 1987.

469. Parra A, Santos D, Cervantes C, et al.: Plasma gonadotropins and gonadal steroids in children treated with cyclophosphamide. J Pediatr 92:117-124, 1978.

470. Puri HC, Campbell RA: Cyclophosphamide and malignancy. Lancet 1:1306, 1977.

471. Scott DGI, Bacon PA: Intravenous cyclophosphamide plus methylprednisolone in treatment of systemic rheumatoid vasculitis. Am J Med 76:377-383, 1984.

472. Pedersen-Bjergaard J, Ersboll J, Hansen VL, et al.: Carcinoma of the urinary bladder after treatment with cyclophosphamide for non-Hodgkin's lymphoma. N Engl J Med 318:1028-1032, 1988. 
473. Arnold MH, Janssen B, Schrieber L, Brooks PM: Prospective pilot study of intravenous pulse cyclophosphamide therapy for refractory rheumatoid arthritis. Arthritis Rheum 32:933-934, 1989.

474. Csuka ME, Carrera GF, McCarty DJ: Treatment of intractable rheumatoid arthritis with combined cyclophosphamide, azathioprine, and hydroxychloroquine. A follow-up study. JAMA 255:2315-2319, 1986.

475. Palmer RG, Varonos S, Dore CJ, et al.: Chlorambucil induced chromosome damage in juvenile chronic arthritis. Arch Dis Child 60:1008-1013, 1985.

476. Palmer RG, Ansell BM: Acute leukaemia related to chlorambucil therapy for juvenile chronic arthritis. Clin Exp Rheumatol 2:81-83, 1984.

477. Callis L, Nieto J, Vila A, et al.: Chlorambucil treatment in minimal lesion nephrotic syndrome: A reappraisal of its gonadal toxicity. J Pediatr 97:653-656, 1980.

478. Cohen AS, Rubinow A, Anderson JJ, et al.: Survival of patients with primary (AL) amyloidosis: colchicine-treated cases from 1978-1983 compared with cases seen in previous years (1961 to 1973). Am J Med 82:1182-1190, 1987.

479. Dhillion V, Woo P, Isenberg D: Amyloidosis in the rheumatic diseases. Ann Rheum Dis 48:696-701, 1989.

480. Shand N, Richardson B: Sandimmun (Cyclosporin A): mode of action and clinical results in rheumatoid arthritis. Scand J Rheumatol 76:265-278, (Suppl) 1988.

481. Bjerkhoel F, Forre O: Cyclosporin treatment of a patient with severe systemic juvenile rheumatoid arthritis. Scand J Rheumatol 17:483-486, 1988.

482. Weinblatt ME, Coblyn JS, Fraser PA, et al.: Cyclosporin A treatment of refractory rheumatoid arthritis. Arthritis Rheum 30:11-17, 1987.

483. Dougados M, Amor B: Cyclosporin A in rheumatoid arthritis: preliminary clinical results of an open trial. Arthritis Rheum 30:83-87, 1987.

484. Forre O, Bjerkhoel F, Salvesen CF, et al.: An open, controlled, randomized comparison of cyclosporine and azathioprine in the treatment of rheumatoid arthritis: a preliminary report. Arthritis Rheum 30:88-92, 1987.

485. Dougados M, Awada H, Amor B: Cyclosporin in rheumatoid arthritis; a double blind, placebo controlled study in 52 patients. Ann Rheum Dis 47:127-133, 1988.

486. Yocum DE, Klippel JH, Wilder RL, et al.: Cyclosporine A in severe treatmentrefractory rheumatoid arthritis. Ann Intern Med 109:863-869, 1988.

487. Tugwell $\mathrm{P}$, Bombardier C, Gent M, et al.: Low-dose cyclosporin versus placebo in patients with rheumatoid arthritis. Lancet 335:1051-5555, 1990.

488. Porter GA, Bennett WM, Sheps SG, et al.: Cyclosporine-associated hypertension. Arch Intern Med 150:280-283, 1990.

489. Oates JA, Wood AJJ, Kahan BD: Drug Therapy. Cyclosporine. N Engl J Med 321:1725-1738, 1990 (see also Letters NEJM 322:1530-1531, 1990.)

490. Gupta AK, Rocher LL, Schmaltz SP, et al.: Short-term changes in renal function, blood pressure, and electrolyte levels in patients receiving cyclosporine for dermatologic disorders. Arch Intern Med 151:356-362, 1991.

491. Ost L: Effects of cyclosporin on prednisolone metabolism. Lancent 1:451, 1984.

492. Whitington PF, Emond JC, Whitington SH, et al.: Small-bowel length and the dose of cyclosporine in children after liver transplantation. 322:733-738, 1990.

493. van Rijthoven AWAM, Dukmans BAC, The DHSG, et al.: Comparison of cyclosporine and D-penicillamine for rheumatoid arthritis: A randomized, double blind, multicenter study. J Rheumatol 18:815-820, 1991. 
493a.Zijlmans JMJM, van Rijthoven AWAM, Kluin PM, et al. Epstein-Barr virusassociated lymphoma in a patient with rheumatoid arthritis treated with cyclosporine. NEJM 326:1363, 1992.

494. Symoens J, Schuermans Y: Levamisole. Clin Rheum Dis 6(Suppl. 5):603-629, 1979.

495. Horsely K, Petersen K, Bentsen KD, Engstrom-Laurent A, et al.: Serum amino terminal Type III procollagen peptide and serum hyaluronan in rheumatoid arthritis: relation to clinical and serological parameters of inflammation during 8 and 24 months' treatment with levamisole, penicillamine or azathioprine. Ann Rheum Dis 47:116-126, 1988.

496. Editorial: Levamisole, a cautionary note. Lancet 2:291-292, 1979.

497. Prieur AM, Buriot D, Lefur JM: Possible toxicity of levamisole in children with rheumatoid arthritis. J Pediatr 93:304-305, 1978.

498. Wallace DJ, Goldfinger D, Lowe C, et al.: A double-blind, controlled study of lymphoplasmapheresis versus sham apheresis in rheumatoid arthritis. $\mathrm{N}$ Eng J Med 306:1406, 1982.

499. Rothwell RS, David P, Gordon PA, et al.: A controlled study of plasma exchange in the treatment of severe rheumatoid arthritis. Arthritis Rheum 23:785-790, 1980.

500. Karsh J, Klippel JH, Plotz PH, et al.: Lymphapheresis in rheumatoid arthritis: A randomized trial. Arthritis Rheum 24:867-873, 1981.

501. Nickleson RW, Brewer EJ, Rossen RD, et al.: Plasma exchange in selected patients with juvenile rheumatoid arthritis. J Pediatr 98:194-200, 1981.

502. Malaise MG, Hauwaert C, Franchimont $P$, et al.: Treatment of active rheumatoid arthritis with slow intravenous injections of thymopentin. A double-blind placebo-controlled randomized study. Lancet 1:832-836, 1985.

503. Riskin WG, Gillings DB, Scarlett JA: Amiprilose hydrochloride for rheumatoid arthritis. Ann Intern Med 111:455-465, 1989.

504. Cannon GW, Emkey RD, Denes A, et al.: Prospective two-year followup of recombinant interferon-gamma in rheumatoid arthritis. J Rheumatol 17:304$310,1990$.

505. Breedveld FC, Dukmans BAC, Mattie H: Minocycline treatment for rheumatoid arthritis: an open dose finding study. J Rheumatol 17:43-46, 1990.

506. Kremer JM, Jubiz W, Michalek A, et al.: Fish-oil fatty acid supplementation in active rheumatoid arthritis. A double-blinded, controlled, crossover study. Ann Intern Med 106:497-503, 1987.

507. Szobor A: Benefit of thymectomy in immune disease other than myasthenia. Lancet 1:277-278, 1984.

508. van Zeben D, Hazes JMW, Vandenbroucke JP, et al.: Diminished incidence of severe rheumatoid arthritis associated with oral contraceptive use. Arthritis Rheum 33:1462-1465, 1990.

509. Pritchard MH, Munro J: Successful treatment of Juvenile Chronic Arthritis with a specific antiviral agent. Br J Rheumatol 28:521-524, 1989.

510. Malawista SE, Trock DH, Edelson RL: Treatment of rheumatoid arthritis by extracorporeal photochemotherapy. A pilot study. Arthritis Rheum 34:646$654,1991$.

511. Southwood TR, Malleson PN, Roberts-Thomson PJ, Mahy M: Unconventional remedies used for patients with juvenile arthritis. Pediatrics 85:150-154, 1990.

512. Jacobs JC: Arthritis as a manifestation of connective tissue diseases. In Moore TD (ed.) Arthritis in Childhood. Report of the 80th Ross Conference on Pediat- 
ric Research. Xanadu, Bahamas, December 2-5, 1979. Ross Laboratories, Columbus, OH, 1981, pp. 18-23.

513. Suciu-Foca N, Jacobs J, Godfrey M, et al.: HLA-DR5 in juvenile rheumatoid arthritis confined to a few joints. Lancet 2:40, 1980.

514. Myers LK, Ball EJ, Nunez G, et al.: HLA-D region epitopes associated with juvenile arthritis. Recognition by alloreactive $\mathrm{T}$ call clones and alloantisera. Arthritis Rheum 30:744-751, 1987.

515. Streekawkas AJ, Collery RT, McDowell J, et al.: Direct evidence for loss of human suppressor cells during active autoimmune disease. Proc Natl Acad Sci 75:5150-5154, 1978.

516. Vostrejs M, Hollister R: Muscle atrophy and leg length discrepancies in pauciarticular juvenile rheumatoid arthritis. Am J Dis Child 142:343-345, 1988.

517. Kanski JJ: Screening for uveitis in juvenile chronic arthritis. Br J Opthalmol 73:225-228, 1989.

518. Fernandez-Vina MA, Fink CW, Stastny P. HLA antigens in juvenile arthritis. Arthritis Rheum 33:1787-1784, 1990.

519. Earley A, Guttica RJ, McCullough CM, Ansell BM: Triamcinolone into the knee joint in juvenile chronic arthritis. Clin Exp Rheumatol 6:153-155, 1988.

520. Balogh K: The histologic appearance of corticosteroid injection sites. Arch Pathol Lab Med 110:1168-1172, 1986.

521. Gilsanz V, Bernstein BH: Joint calcification following intraarticular corticosteroid therapy. Radiology 151:647-649, 1984.

522. Sparling M, Malleson P, Wood B, Petty R: Radiographic followup of joints injected with triamcinolone hexacetonide for the management of childhood arthritis. Arthritis Rheum 33:821-826, 1990.

523. Allen RC, Gross KR, Malleson PN, et al.: Intraarticular triamcinolone hexacetonide in the management of chronic arthritis in children. Arthritis Rheum 29:997-1001, 1986.

524. Huskisson EC: Intraarticular and soft-tissue injections. In Hart FD (ed.) Drug Treatment of the Rheumatic Diseases Williams and Wilkins, Sydney, 1987, pp. 111-116.

525. Montgomery SC, Campbell J: Septic arthritis following arthroscopy and intraarticular steroids. J Bone Joint Surg 71-8:540, 1989.

526. Hutchinson J: On a peculiar form of iritis which occurs in the children of gouty parents. Lancet 1-3, 1873.

527. Calabro JJ, Marchesano JM, Parrino GR: Juvenile rheumatoid arthritis: longterm management and prognosis. J Musculoskeletal Med pp. 17-32, January 1989.

528. Sherry DD, Mellins ED, Wedgwood RJ: Decreasing severity of chronic uveitis in children with pauciarticular arthritis. Am J Dis Child 145:1026-1028. 1991.

529. Spalter HF: The visual prognosis in juvenile rheumatoid arthritis. Trans Am Ophthalmol Soc 73:554-570, 1975.

530. Kanski JJ: Uveitis in juvenile chronic arthritis: incidence, clinical features and prognosis. Eye 2:641-645, 1988.

531. Melin-Aldana H, Giannini EH, Taylor J, et al:: Human leukocyte antigenDRBI*1104 in the chronic iridocyclitis of pauciarticular juvenile rheumatoid arthritis. J Pediatr 121:56-60, 1992.

532. Rosenberg AM, Oen KG: The relationship between ocular and articular disease 
activity in children with juvenile rheumatoid arthritis and associated uveitis. Arthritis Rheum 29:797-800, 1986.

533. Olson NY, Lindsley CB, Godfrey WA: Nonsteriodal anti-inflammatory drug therapy in chronic childhood iridocyclitis. Am J Dis Child 142:1289-1292, 1988.

533a.Dougados M, Berenbaum F, Maetzel A, et al.: The use of sulfasalazine for the prevention of attacks of acute anterior uveitis associated with spondyloarthropathy. Arthritis Rheum 34:S195, 1991.

534. Chylack LT, Dueker DK, Pihlaja DJ: Ocular manifestations of juvenile rheumatoid arthritis: Pathology, florescein iris angiography, and patient care patterns. In Miller JJ III (ed.) Juvenile Rheumatoid Arthritis. PSG, Littleton, MA, 1979.

535. Johns KJ, Chandra SR: Visual loss following intranasal corticosteroid injection. JAMA 261:2413, 1989.

536. Merrimam JC, Chylack LT, Albert DM: Early-onset pauciarticular juvenile rheumatoid arthritis: a histopathologic study. Arch Ophthalmol 101:10851092, 1983.

537. Wolf MD, Lichter PR, Ragsdale CG: Prognostic factors in the uveitis of juvenile rheumatoid arthritis. Opththalmology 94:1242-1248, 1987.

538. Herman DC: Endogenous Uveitis: Current concepts of treatment. Mayo Clin Proc 65:671-683, 1990.

539. Palmer RG, Kanski JJ, Ansell BM: Chlorambucil in the treatment of intractable uveitis associated with juvenile chronic arthritis. J Rheumatol 12:967-970, 1985.

540. Palestine AG, Austin HA III, Balow JE, et al.: Renal histopathologic alterations in patients treated with cyclosporine for uveitis. N Engl J Med 314:1293$1298,1986$.

540a. Vawter RL, Macsai M, Fakadej A, et al. Methotrexate for inflammatory eye disease. Arthritis rheum 35 \#5S:R12, 1992.

540b.Bridges AJ, Burns RP. Acute iritis associated with primary Sjogren's syndrome and high-titer anti-SS-A/Ro and anti-SS-B-/La antibodies. Athritis Rheum 35:560-563, 1992.

541. Kanski JJ: Juvenile arthritis and uveitis. Surv Ophthalmol 34:253-267, 1990.

542. Kanski JJ, Shun-Shin GA: Systemic uveitis syndromes in childhood: an analysis of 340 cases. Ophthamology 91:1247-1252, 1984.

543. Praeger DL, Schneider HA, Sakowski AD, Jacobs JC: Kelman procedure in the treatment of complicated cataract of uveitis of Still's disease. Trans Ophthalmol Soc UK 96:168-172, 1976.

544. Flynn HW, Davis JL, Culbertson WW, Pars plana lensectomy and vitrectomy for complicated cataracts in juvenile rheumatoid arthritis. Ophthalmology 95:1114-1119, 1988.

545. Diamond JG, Kaplan HJ: Lensectomy and vitrectomy for complicated cataract secondary to uveitis. Arch Ophthalmol 96:1798-1804, 1978.

546. Lambert JR, Ansell BM, Stephenson E, et al.: Psoriatic arthritis in childhood. Clin Rheum Dis 2:339-352, 1976.

547. Sills EM: Psoriatic arthritis in childhood. Johns Hopkins Med J 146:49-53, 1980.

548. Shore A, Ansell BM: Juvenile psoriatic arthritis-an analysis of 60 cases. 
J Pediatr 100:529-535, 1982.

549. Southwood TR, Petty RS, Malleson PN, et al.: Psoriatic arthritis in children. Arthritis Rheum 32:1007-1013, 1989.

550. Gerber J, Espinoza LR (eds.) Psoriatic Arthritis. Grune and Stratton, Orlando, 1985.

551. Seideman P, Fjellner B, Johannesson A: Psoriatic arthritis treated with oral colchicine. J Rheumatol 14:777-779, 1987.

552. Steinsson K, Jonsdottir I, Valdimarsson H: Cyclosporin A in psoriatic arthritis: an open study. Ann Rheum Dis 49:603-606, 1990.

553. Huckins D, Felson DT, Holick M: Treatment of psoriatic arthritis with oral 1, 25-dihydroxyvitamin D3: A pilot study. Arthritis Rheum 33:1723-1727, 1990.

554. Ellis CN, Fradin MS, Messana JM, et al.: Cyclosporine for plaque-type psoriasis. Results of a multidose, double-blind trial. N Engl J Med 324:277-294, 1991 (see also correspondence pp. 1894-1985).

555. Randall T: Cyclosporine: vital in today's transplantation, but questions remain about tomorrow. JAMA 264:1794-1797, 1990.

556. van Rijthoven AWAM, Dijkmans BAC, Goei The HS, et al.: Long-term cyclosporine therapy in rheumatoid arthritis. J Rheumatol 18:19-23, 1991.

557. Gay RE, Palmoski MJ, Brandt KD, Gay S: Aspirin causes in vivo synthesis of type I collagen by atrophic articular cartilage. Arthritis Rheum 26:1231-1236, 1983.

558. Williams PE: Effect of intermittent stretch on imobilized muscle. Ann Rheum Dis 47:1014-1016, 1988.

559. Hinojosa J, Anderson J, Strauch C: Pediatric occupational therapy in the home. Am J Occup Ther 42:17-22, 1988.

560. Gallino L, Pountain G, Mitchell N, Ansell BM: Developmental aspects of the hip in juvenile chronic arthritis. A radiologic assessment. Scand J Rhematol 13:310-318, 1984.

561. Vostrejs M, Hollister JR: Muscle atrophy and leg length discrepancies in pauciarticular juvenile rheumatoid arthritis. Am J Dis Child 142:343-345, 1988.

562. Editorial: joint injury and muscle weakness. Lancet 2:381-382, 1984.

563. Jacobsen ST, Levinson JE, Crawford AH: Late results of synovectomy in juvenile rheumatoid arthritis. J Bone Joint Surg 67-A:8-15, 1985.

564. Rydholm U, Elborgh R, Ranstam J, et al.: Synovectomy of the knee in juvenile chronic arthritis: a retrospective, consecutive follow-up study. J Bone Joint Surg 68B:223-228, 1986.

564a.Ovregard T, Hoyeraal HM, Pahle JA, Larsen S. A three-year retrospective study of synovectomies in children. Clin Orthop Rel Res 259:76-82, 1990.

565. Combe B, Krause E, Sany J: Treatment of chronic knee-synovitis with arthroscopic synovectomy after failure of intraarticular injection of radionuclide. Arthritis Rheum 32:10-14, 1989.

566. Mogensen B, Brattstrom H, Svantesson H, Lidgren L: Soft tissue release of the hip in juvenile chronic arthritis. Scand J Rheumatol 12:17-20, 1983.

567. Scott RD, Sarokhan AJ, Dalziel R: Total hip and total knee arthroplasty in juvenile rheumatoid arthritis. Clin Orthop 182:90-97, 1984.

568. Kunec JR: Total hip replacement in patients under thirty-five years of age. Orthopedics 6:1432-1434, 1983.

569. Ruddlesdin C, Ansell BM, Arden GP, Swann M: Total hip replacement in 
children with juvenile chronic arthritis. J Bone Joint Surg 68B:218-222, 1986.

570. Arthritis of the hip in children. Lancet 2:260, 1986.

571. Gudmundsson GH, Harving S, Pilgaard S: The Charnley total hip arthroplasty in juvenile rheumatoid arthritis patients. Orthopedics 12:385-388, 1989.

572. Dossick PH, England S, Huo M, et al.: Total hip replacement in juvenile rheumatoid arthritis. Arthritis Rheum 33 \#5(Suppl):R40, 1990.

573. Sarokhan AJ, Scott RD, Thomas WH, et al.: Total knee arthroplasty in juvenile rheumatoid arthritis. J Bone Joint Surg 65A:1071-1080, 1983.

574. Nicholas SA, Figgie MP, Alexiades MM, et al.: Total knee replacement in juvenile rheumatoid arthritis. Arthritis Rheum 33 \#5(Suppl):R40, 1990. 